\title{
Gold-catalyzed hydrofluorination of internal alkynes using aqueous $\mathrm{HF}$
}

\author{
Raphaël Gauthier, Marius Mamone and Jean-François Paquin* \\ CGCC, PROTEO, Département de chimie, Université Laval, 1045 avenue de la \\ Médecine, Québec, Québec, G1V 0A6, Canada \\ *Corresponding author: jean-francois.paquin@chm.ulaval.ca
}

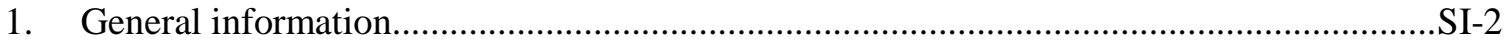

2. General procedures for the synthesis of alkynes .............................................................

Procedure A: Preparation of diaryl-alkynes by Sonogashira coupling ..................................SI-3

Procedure B: Preparation of symmetrical diaryl-alkynes by modified Sonogashira coupling conditions

SI-3

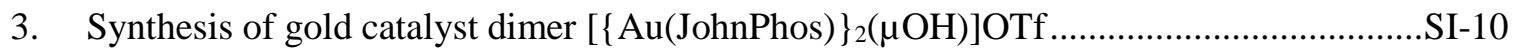

4. General procedures for the hydrofluorination of alkynes ................................................SI-11

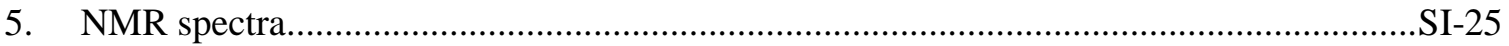




\section{General information}

The following includes general experimental procedures, specific details for representative reactions, isolation and spectroscopic information for the new compounds prepared. All commercial compounds were used as received. Solvents were used as purchased unless stated as dry. THF, $\mathrm{CH}_{2} \mathrm{Cl}_{2}$ and toluene were purified using a Vacuum Atmospheres Inc. Solvent Purification System. All air and water sensitive reactions were carried out under argon atmosphere. Reactions were monitored by TLC on pre-coated plates (Silicycle silica gel 60 A F254 230-240 mesh) and products were visualized under $254 \mathrm{~nm}$ UV light followed by staining with $\mathrm{KMnO}_{4}, \mathrm{CAM}$ or PMA when appropriate. Purification by flash column chromatography was carried out on silica gel (Silicycle silica gel $60 \AA$ F254) or was carried out under Biotage ${ }^{\circledR}$ Isolera One Flash Chromatography System using SiliaSep silica gel cartridge or Biotage ${ }^{\circledR}$ SNAP KP-Sil cartridges. NMR spectra were recorded on an Agilent DD2 500 spectrometer or on a Varian Inova 400 spectrometer in the indicated solvent at $298 \mathrm{~K}$. Chemical shifts for ${ }^{1} \mathrm{H}$ and ${ }^{13} \mathrm{C}$ spectra are reported on the delta scale in ppm and were referenced to TMS reference. For ${ }^{19} \mathrm{~F}$ and ${ }^{31} \mathrm{P} \mathrm{NMR} \mathrm{CFCl}_{3}$ and phosphoric acid are respectively used as external standards. Resonances are reported as follows: chemical shift $(\delta$ ppm $)$, multiplicity $(\mathrm{s}=$ singulet, $\mathrm{d}=$ doublet, $\mathrm{t}=$ triplet, $\mathrm{q}=$ quartet, $\mathrm{p}=$ quintet, $\mathrm{m}=$ multiplet, br. $\mathrm{s}=$ broad signal), coupling constant $(\mathrm{Hz})$, integration. NMR yields were determined by ${ }^{19} \mathrm{~F}$ NMR analysis of the crude mixture after work-up using 2fluoro-4-nitrotoluene as the internal standard. High-resolution mass (HRMS) spectra were obtained on a LC/MS-TOF Agilent 6210 using electrospray ionization (ESI) and atmospheric pressure photoionization (APPI). Low resolution mass spectra (GC-MS) were obtained on a GC-MS Thermo ITQ 900 Trace GC Ultra using electron ionization (EI). Infrared spectra were recorded on an ABB MB3000 FT-IR spectrometer. Melting points were measured on a Stanford Research System OptiMelt MPA100 automated melting point 
apparatus and are uncorrected. Substrates $1 \mathbf{b},{ }^{1} 1 \mathbf{c},{ }^{1} 1 \mathbf{d},{ }^{1} 1 \mathbf{h},{ }^{1} \mathbf{1 k},{ }^{2} \mathbf{1 w},{ }^{3} 3 \mathbf{3},{ }^{4} 3 \mathbf{b}^{5}$ and $3 \mathbf{c}^{5}$ were prepared according to literature procedures. Aqueous HF can be very dangerous if it is swallowed, inhaled or if it enters in contact with skin. Special precautions, as indicated in the Material Safety Data Sheet, should be taken when handling this reagent.

\section{General procedures for the synthesis of alkynes}

\section{Procedure A: Preparation of diaryl-alkynes by Sonogashira coupling}

In a round-bottomed flask, the aryl iodide or bromide (1 equiv.) is dissolved in argonsparged triethylamine $(0.3 \mathrm{M})$. The arylacetylene (1.1 equiv.) is added, followed by bis(triphenylphosphine)palladium (II) dichloride (3 mol \%) and copper (I) iodide (6 mol $\%)$. After 18 hours at $60{ }^{\circ} \mathrm{C}$, the solvent is evaporated, and the crude product is purified on flash silica gel chromatography.

\section{Procedure B: Preparation of symmetrical diaryl-alkynes by modified Sonogashira}

\section{coupling conditions}

Following a procedure reported by Milo et al. ${ }^{1}$ : In a round-bottomed flask, the aryl iodide, bromide or triflate (1 equiv.) is dissolved in argon-sparged benzene $(0.2 \mathrm{M})$. Bis(triphenylphosphine)palladium (II) dichloride (6 mol \%) and copper (I) iodide (10 mol $\%)$ are added, followed by 1,8-diazabicyclo(5.4.1)undec-7-ene (6 equiv.), ethynyltrimethylsilane ( 0.5 equiv.) and water $(40 \mathrm{~mol} \%)$. The reaction is covered in aluminum foil and stirred upon completion determined by TLC. Reaction mixture is partitioned in EtOAc and water. The aqueous layer is extracted with EtOAc (2x) and the combined organic layers are washed with $3 \mathrm{~N} \mathrm{HCl} \mathrm{(3x)} \mathrm{and} \mathrm{brine,} \mathrm{dried} \mathrm{over} \mathrm{Na}_{2} \mathrm{SO}_{4}$, filtered and evaporated in vacuo. The crude product is purified on flash silica gel chromatography.

\footnotetext{
${ }^{1}$ Milo, M.J.; Kopel, L.C.; Braun, J.B.; Gadzikwa, T.L.; Hull, K.L.; Brisbois, R.G.; Markworth, C.J.; Grieco, P.A., Org. Lett. 2002, 4, 3199-3202.

${ }^{2}$ Nahra, F.; Patrick, S.R.; Bello, D.; Brill, M.; Obled, A.; Cordes, D.B.; Slawin, A.M.Z.; O'Hagan, D.; Nolan, S.P., ChemCatChem 2014, 7, 1174-1182.

${ }^{3}$ Sahini, R.L.; Liu, R.-S., Angew. Chem. Int. Ed. 2017, 56, 12736-12740.

${ }^{4}$ Hamel, J.-D.; Hayashi, T.; Cloutier, M.; Savoie, P.R.; Thibeault, O.; Beaudoin, M.; Paquin, J.-F. Org. Biomol. Chem. 2017, 15, 9830-9836.

${ }^{5}$ Cloutier, M.; Roudias, M.; Paquin, J.-F., Org. Lett. 2019, 21, 3866-3870.
} 
$\mathrm{MeO}$

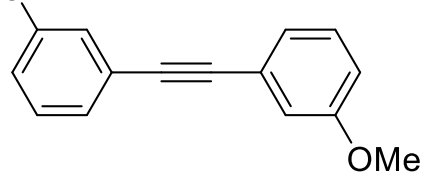

\section{1,2-bis(3-Methoxyphenyl)ethyne (1e)}

Prepared according to the general procedure B on a 3.08

mmol scale, the desired product (121 mg, $0.51 \mathrm{mmol}, 34 \%$ )

was isolated as a white solid after purification by flash column chromatography (25-50\% toluene in hexanes). ${ }^{1} \mathrm{H}$ NMR $\left(400 \mathrm{MHz}, \mathrm{CDCl}_{3}\right) \delta(\mathrm{ppm})=7.29-7.21(\mathrm{~m}, 2 \mathrm{H}), 7.17-7.10$ (m, 2H), $7.06(\mathrm{dd}, J=2.6,1.4 \mathrm{~Hz}, 2 \mathrm{H}), 6.89$ (ddd, $J=8.3,2.6,1.0 \mathrm{~Hz}, 2 \mathrm{H}) 3.82(\mathrm{~s}, 6 \mathrm{H})$. Data are in accordance with the one described in the literature. ${ }^{6}$

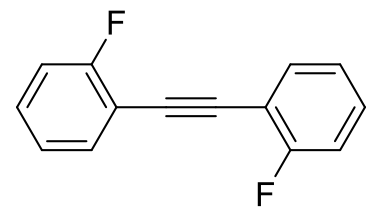

\section{1,2-bis(2-Fluorophenyl)ethyne (1f)}

Prepared according to the general procedure B on a $2.14 \mathrm{mmol}$ scale, the desired product (148 $\mathrm{mg}, 0.69 \mathrm{mmol}, 66 \%$ ) was isolated as a white solid after purification by automated flash purification (hexanes). Mp: 53.7-54.7 ${ }^{\circ} \mathrm{C}$; FT-IR $\vee\left(\mathrm{cm}^{-1}\right)=1576,1501,1483,1452,1263,1229,1217,824,777,750 ;{ }^{1} \mathrm{H}$ NMR $\left(400 \mathrm{MHz}, \mathrm{CDCl}_{3}\right) \delta(\mathrm{ppm})=7.57-7.50(\mathrm{~m}, 2 \mathrm{H}), 7.36-7.29(\mathrm{~m}, 2 \mathrm{H}), 7.16-7.06(\mathrm{~m}, 4 \mathrm{H})$; ${ }^{13} \mathrm{C}$ NMR $\left(126 \mathrm{MHz}, \mathrm{CDCl}_{3}\right) \delta(\mathrm{ppm})=162.7\left(\mathrm{~d}, J_{C-F}=252.3 \mathrm{~Hz}\right), 133.6\left(\mathrm{~d}, J_{C-F}=1.2\right.$ $\mathrm{Hz}), 130.5\left(\mathrm{~d}, J_{C-F}=8.0 \mathrm{~Hz}\right), 124.1\left(\mathrm{~d}, J_{C-F}=3.8 \mathrm{~Hz}\right), 115.7\left(\mathrm{~d}, J_{C-F}=20.8 \mathrm{~Hz}\right), 111.7(\mathrm{~d}$, $\left.J_{C-F}=15.5 \mathrm{~Hz}\right), 87.8\left(\mathrm{~d}, J_{C-F}=3.2 \mathrm{~Hz}\right) ;{ }^{19} \mathrm{~F} \mathrm{NMR}\left(470 \mathrm{MHz}, \mathrm{CDCl}_{3}\right) \delta(\mathrm{ppm})=-109.6(\mathrm{~m}$, 2F); HRMS (APPI-TOF) $\mathrm{m} / \mathrm{z}$ calcd for $\mathrm{C}_{14} \mathrm{H}_{8} \mathrm{~F}_{2}\left[\mathrm{M}^{*}\right]^{+} 214.0600$; found 214.0589.

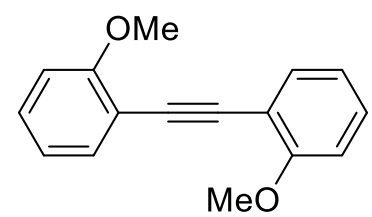

\section{1,2-bis(2-Methoxyphenyl)ethyne (1g)}

Prepared according to the general procedure B on a $2.99 \mathrm{mmol}$ scale, the desired product $(53 \mathrm{mg}, 0.22 \mathrm{mmol}, 16 \%)$ was isolated as a beige solid after purification by automated flash purification (0-5\% EtOAc in hexanes). ${ }^{1} \mathrm{H}$ NMR $\left(400 \mathrm{MHz}, \mathrm{CDCl}_{3}\right) \delta(\mathrm{ppm})=7.53(\mathrm{dd}, J=7.6,1.7 \mathrm{~Hz}, 2 \mathrm{H}), 7.29(\mathrm{ddd}, J=8.2$, 7.4, $1.7 \mathrm{~Hz}, 2 \mathrm{H}), 6.96-6.87(\mathrm{~m}, 4 \mathrm{H}), 3.92(\mathrm{~s}, 6 \mathrm{H})$. Data are in accordance with the one described in the literature. ${ }^{7}$

\footnotetext{
${ }^{6}$ Zhang, W.; Huayue, W.; Liu, Z.; Zhong, P.; Zhang, L.; Huang, X.; Cheng, J., Chem. Commun. 2006, 46, $4826-4828$.

${ }^{7}$ Sashuk, V.; Ignatowska, J.; Grela, K., J. Org. Chem. 2004, 69, 7748-7751.
} 


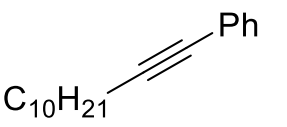

\section{Dodec-1-yn-1-ylbenzene (1k)}

Following procedure from Nahra et al. ${ }^{2} n$-Buthyllithium $(1.45 \mathrm{~mL}$ of a 2.5 M solution in hexanes, $3.63 \mathrm{mmol}, 1$ equiv.) is added dropwise on a phenylacetylene (0.48 mL, $4.37 \mathrm{mmol}, 1.2$ equiv.) solution in dry THF $(25 \mathrm{~mL}, 0.2 \mathrm{M})$ cooled to $-78{ }^{\circ} \mathrm{C}$. Dry ice bath is removed, and the reaction is allowed to warm to room temperature. 1Bromodecane $(0.75 \mathrm{~mL}, 3.62 \mathrm{mmol}, 1$ equiv.) and tetrabutylammonium iodide (139 mg, $0.38 \mathrm{mmol}, 0.1$ equiv.) are added and the reaction is heated to reflux for 22 hours. The reaction is cooled to room temperature and water is added. Reaction mixture is extracted with $\mathrm{Et}_{2} \mathrm{O}(3 \mathrm{x})$. Combined organic layers are washed with brine, dried over $\mathrm{Na}_{2} \mathrm{SO}_{4}$, filtered and evaporated in vacuo. The desired product (453 mg, $1.87 \mathrm{mmol}, 52 \%$ ) was isolated as a colourless oil after purification by automated flash purification (hexanes). ${ }^{1} \mathrm{H}$ $\operatorname{NMR}\left(400 \mathrm{MHz}, \mathrm{CDCl}_{3}\right) \delta(\mathrm{ppm})=7.43-7.36(\mathrm{~m}, 2 \mathrm{H}), 7.32-7.23(\mathrm{~m}, 3 \mathrm{H}), 2.40(\mathrm{t}, J=7.1$ $\mathrm{Hz}, 2 \mathrm{H}), 1.67-1.55(\mathrm{~m}, 2 \mathrm{H}), 1.50-1.38(\mathrm{~m}, 2 \mathrm{H}), 1.36-1.20(\mathrm{~m}, 12 \mathrm{H}), 0.88(\mathrm{t}, J=6.7 \mathrm{~Hz}$, $3 \mathrm{H})$. Data are in accordance with the one described in the literature. ${ }^{8}$

6-Phenylhex-5-yn-1-ol
In a round-bottomed flask, iodobenzene $(0.45 \mathrm{~mL}, 4.02 \mathrm{mmol}, 1$ equiv.) is dissolved in argon-sparged triethylamine (10 mL, 0.4 M). 5-Hexyn-1-ol (0.49 $\mathrm{mL}, 4.44 \mathrm{mmol}, 1.1$ equiv.) is added, followed by bis(triphenylphosphine)palladium (II) dichloride (42 mg, $0.06 \mathrm{mmol}, 1.5 \mathrm{~mol} \%$ ) and copper (I) iodide (25 mg, $0.15 \mathrm{mmol}, 3.8$ mol \%). After 18 hours at room temperature, the solvent is evaporated. The desired product (694 mg, $3.98 \mathrm{mmol}, 99 \%$ ) was isolated as an orange oil after purification by automated flash purification (10-20\% EtOAc/hexanes). ${ }^{1} \mathrm{H} \mathrm{NMR}\left(500 \mathrm{MHz}, \mathrm{CDCl}_{3}\right) \delta(\mathrm{ppm})=7.41-$ $7.36(\mathrm{~m}, 2 \mathrm{H}), 7.30-7.23(\mathrm{~m}, 3 \mathrm{H}), 3.69(\mathrm{td}, J=6.3,1.5 \mathrm{~Hz}, 2 \mathrm{H}), 2.45(\mathrm{td}, J=6.8,0.9 \mathrm{~Hz}$, $2 \mathrm{H}), 1.79-1.60(\mathrm{~m}, 5 \mathrm{H})$. Data are in accordance with the one described in the literature. ${ }^{9}$

\footnotetext{
${ }^{8}$ Yasukawa, T.; Miyamura, H.; Kobayashi, S. Org. Biomol. Chem. 2011, 9, 6208-6210.

${ }^{9}$ Korner, C.; Starkov, P.; Sheppard, T.D., J. Am. Chem. Soc. 2010, 132, 5968-5969.
} 
6-Phenylhex-5-yn-1-yl acetate (11)
4-Dimethylaminopyridine $(8 \mathrm{mg}, 0.07 \mathrm{mmol}, 0.1$ equiv.) was added on a solution of 6-phenylhex-5-yn-1-ol (108 mg, $0.62 \mathrm{mmol}, 1$ equiv.) and acetic anhydride $(0.29 \mathrm{~mL}, 3.07 \mathrm{mmol}, 5$ equiv. $)$ in pyridine $(2 \mathrm{~mL}, 0.3 \mathrm{M})$ and the reaction mixture was stirred for 2.5 hours at room temperature. Icy water was added and the aqueous layer was extracted with $\mathrm{CH}_{2} \mathrm{Cl}_{2}(3 \mathrm{x})$. Combined organic layers were washed with saturated $\mathrm{NaHCO}_{3}(2 \mathrm{x})$, dried over $\mathrm{Na}_{2} \mathrm{SO}_{4}$, filtered and evaporated in vacuo. The desired product (113 mg, $0.52 \mathrm{mmol}, 85 \%)$ was isolated as a colourless oil after purification by automated flash purification (0-10\% EtOAc/hexanes). FT-IR $v\left(\mathrm{~cm}^{-1}\right)=2951,1736,1599$, $1491,1433,1387,1366,1234,1045,756 ;{ }^{1} \mathrm{H}$ NMR $\left(500 \mathrm{MHz}, \mathrm{CDCl}_{3}\right) \delta(\mathrm{ppm})=7.42-$ $7.37(\mathrm{~m}, 2 \mathrm{H}), 7.31-7.24(\mathrm{~m}, 3 \mathrm{H}), 4.12(\mathrm{t}, J=6.5 \mathrm{~Hz}, 2 \mathrm{H}), 2.46(\mathrm{t}, J=7.0 \mathrm{~Hz}, 2 \mathrm{H}), 2.06$ (s, $3 \mathrm{H}), 1.86-1.76(\mathrm{~m}, 2 \mathrm{H}), 1.73-1.62(\mathrm{~m}, 2 \mathrm{H}) ;{ }^{13} \mathrm{C} \mathrm{NMR}\left(126 \mathrm{MHz}, \mathrm{CDCl}_{3}\right) \delta(\mathrm{ppm})=171.2$, 131.5, 128.2 127.6, 123.8, 89.5, 81.1, 64.0, 27.8, 25.2, 21.0, 19.1; HRMS (ESI-TOF) m/z calcd for $\mathrm{C}_{14} \mathrm{H}_{16} \mathrm{O}_{2}[\mathrm{M}+\mathrm{H}]^{+} 217.1223$; found 217.1212 .

$\mathrm{Ph}$ 6-Phenylhex-5-yn-1-yl benzoate (1m)
A solution of 6-phenylhex-5-yn-1-ol $(103 \mathrm{mg}, 0.59 \mathrm{mmol}, 1$ equiv.) and triethylamine (0.10 mL, $0.72 \mathrm{mmol}, 1.2$ equiv.) in dry $\mathrm{CH}_{2} \mathrm{Cl}_{2}(3 \mathrm{~mL}, 0.2 \mathrm{M})$ was cooled to $0{ }^{\circ} \mathrm{C}$ under argon atmosphere. Benzoyl chloride $(80 \mu \mathrm{L}, 0.69 \mathrm{mmol}, 1.2$ equiv.) was added dropwise and the reaction mixture was allowed to warm to room temperature. After 20 hours, $1 \mathrm{M} \mathrm{HCl}_{(\mathrm{aq})}$ was added and the aqueous layer was extracted with $\mathrm{CH}_{2} \mathrm{Cl}_{2}(3 \mathrm{x})$. Combined organic layers were washed with water $(2 \mathrm{x})$, dried over $\mathrm{Na}_{2} \mathrm{SO}_{4}$, filtered and evaporated in vacuo. The desired product (151 mg, $0.54 \mathrm{mmol}, 92 \%$ ) was isolated as a colourless oil after purification by flash column chromatography (3\% EtOAc/hexanes). FT-IR $v\left(\mathrm{~cm}^{-1}\right)=2951,1717,1601,1491,1313,1271,1115,1070,1026$, $710 ;{ }^{1} \mathrm{H}$ NMR $\left(400 \mathrm{MHz}, \mathrm{CDCl}_{3}\right) \delta(\mathrm{ppm})=8.06(\mathrm{~d}, J=7.8 \mathrm{~Hz}, 2 \mathrm{H}), 7.59-7.52(\mathrm{~m}, 1 \mathrm{H})$, 7.46-7.37 (m, 4H), 7.31-7.20 (m, 3H), $4.38(\mathrm{t}, J=6.4 \mathrm{~Hz}, 2 \mathrm{H}), 2.51(\mathrm{t}, J=7.0 \mathrm{~Hz}, 2 \mathrm{H})$, 2.03-1.91 (m, 2H), 1.83-1.73 (m, 2H); $\left.{ }^{13} \mathrm{C} \mathrm{NMR} \mathrm{(101} \mathrm{MHz,} \mathrm{CDCl}_{3}\right) \delta(\mathrm{ppm})=166.6$, 132.9, 131.6, 130.4, 129.6, 128.2, 127.6, 123.8, 89.5, 81.1, 64.6, 28.0, 25.4, 19.1; HRMS (ESI-TOF) $\mathrm{m} / \mathrm{z}$ calcd for $\mathrm{C}_{19} \mathrm{H}_{19} \mathrm{O}_{2}[\mathrm{M}+\mathrm{H}]^{+} 279.1380$; found 279.1354. 


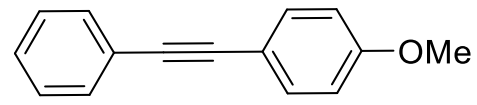

\section{1-Methoxy-4-(phenylethynyl)benzene (1n)}

Prepared according to general procedure A on a $0.87 \mathrm{mmol}$ scale, the desired product (129 $\mathrm{mg}, 0.62 \mathrm{mmol}, 87 \%$ ) was isolated as a white solid after purification by flash column chromatography (hexanes). ${ }^{1} \mathrm{H} \mathrm{NMR}\left(400 \mathrm{MHz}, \mathrm{CDCl}_{3}\right) \delta$ $(\mathrm{ppm})=7.57-7.43(\mathrm{~m}, 4 \mathrm{H}), 7.38-7.26(\mathrm{~m}, 3 \mathrm{H}), 6.92-6.83(\mathrm{~m}, 2 \mathrm{H}), 3.82(\mathrm{~s}, 3 \mathrm{H})$. Data are in accordance with the one described in the literature. ${ }^{10}$

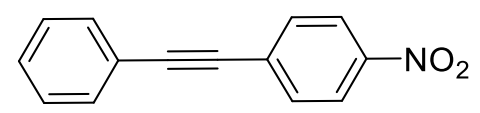

\section{1-Nitro-4-(phenylethynyl)benzene (10)}

4-Nitrophenyl trifluoromethanesulfonate (202 $\mathrm{mg}, 0.75$ mmol, 1 equiv.) is dissolved in argon-sparged DMF (7.5 mL, 0.1 M). Phenylacetylene ( $0.10 \mathrm{~mL}, 0.91 \mathrm{mmol}, 1.2$ equiv. $)$ is added, followed by triethylamine $(0.21 \mathrm{~mL}, 1.51 \mathrm{mmol}$, 2 equiv.), lithium chloride (64 mg, $1.51 \mathrm{mmol}, 2$ equiv.), bis(triphenylphosphine)palladium (II) dichloride (27 mg, $0.04 \mathrm{mmol}, 5 \mathrm{~mol} \%$ ) and copper (I) iodide (14 mg, $0.07 \mathrm{mmol}, 10$ mol\%). The reaction is stirred at $80{ }^{\circ} \mathrm{C}$ for $24 \mathrm{~h}$. The reaction mixture is partitioned in EtOAc and water. Aqueous layer is extracted with EtOAc and the combined organic layers are washed with brine (3x), dried over $\mathrm{Na}_{2} \mathrm{SO}_{4}$, filtrated and evaporated in vacuo. Desired product (115 mg, $0.52 \mathrm{mmol}, 69 \%$ ) was isolated as a orange solid after purification by automated flash purification (0-5\% EtOAc in hexanes). ${ }^{1} \mathrm{H}$ NMR $\left(400 \mathrm{MHz}, \mathrm{CDCl}_{3}\right) \delta$ $(\mathrm{ppm})=8.26-8.18(\mathrm{~m}, 2 \mathrm{H}), 7.70-7.64(\mathrm{~m}, 2 \mathrm{H}), 7.59-7.53(\mathrm{~m}, 2 \mathrm{H}), 7.43-7.36(\mathrm{~m}, 3 \mathrm{H})$. Data are in accordance with the one described in the literature. ${ }^{10}$

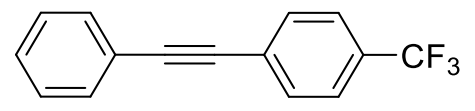

\section{1-(Phenylethynyl)-4-(trifluoromethyl)benzene (1p)}

Prepared according to general procedure A using 4bromobenzotrifluoride on a $1.50 \mathrm{mmol}$ scale, the desired product $(295 \mathrm{mg}, 1.20 \mathrm{mmol}$, $80 \%$ ) was isolated as a white solid after purification by flash column chromatography (hexanes). ${ }^{1} \mathrm{H}$ NMR $\left(500 \mathrm{MHz}, \mathrm{CDCl}_{3}\right) \delta(\mathrm{ppm})=7.66-7.58(\mathrm{~m}, 4 \mathrm{H}), 7.57-7.52(\mathrm{~m}, 2 \mathrm{H})$, 7.40-7.38 (m, 3H); ${ }^{19} \mathrm{~F} \mathrm{NMR}\left(470 \mathrm{MHz}, \mathrm{CDCl}_{3}\right) \delta(\mathrm{ppm})=-62.7(\mathrm{~s}, 3 \mathrm{~F})$. Data are in accordance with the one described in the literature. ${ }^{11}$

\footnotetext{
${ }^{10}$ Park, S.B.; Alper, H., Chem. Comm. 2004, 11, 1306-1307.

11 Thakur, K. G.; Sekar, G., Synthesis 2009, 16, 2785-2789.
} 


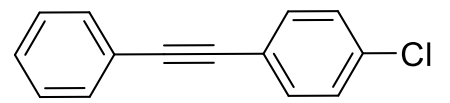

\section{1-Chloro-4-(phenylethynyl)benzene (1q)}

Prepared according to general procedure A using 4'iodoacetophenone on a $1.49 \mathrm{mmol}$ scale, the desired product (298 $\mathrm{mg}, 1.40 \mathrm{mmol}, 94 \%$ ) was isolated as a white solid after purification by flash column chromatography (hexanes). ${ }^{1} \mathrm{H}$ NMR $\left(500 \mathrm{MHz}, \mathrm{CDCl}_{3}\right) \delta(\mathrm{ppm})=7.56-7.47(\mathrm{~m}, 2 \mathrm{H}), 7.49-7.42(\mathrm{~m}, 2 \mathrm{H}), 7.39-7.26$ $(\mathrm{m}, 5 \mathrm{H})$. Data are in accordance with the one described in the literature. ${ }^{12}$

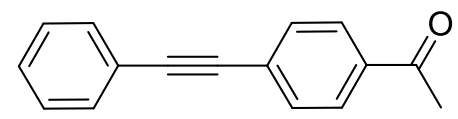

\section{1-(4-(Phenylethynyl)phenyl)ethan-1-one (1r)}

Prepared according to general procedure A using 4'iodoacetophenone on a $1.50 \mathrm{mmol}$ scale, the desired product ( $271 \mathrm{mg}, 1.23 \mathrm{mmol}, 82 \%$ ) was isolated as a yellow solid after purification by flash column chromatography $(10 \%$ EtOAc in hexanes). ${ }^{1} \mathrm{H}$ NMR $\left(400 \mathrm{MHz}, \mathrm{CDCl}_{3}\right) \delta(\mathrm{ppm})=7.98-7.90(\mathrm{~m}, 2 \mathrm{H}), 7.65-7.57$ $(\mathrm{m}, 2 \mathrm{H}), 7.60-7.50(\mathrm{~m}, 2 \mathrm{H}), 7.42-7.31(\mathrm{~m}, 3 \mathrm{H}), 2.61(\mathrm{~s}, 1 \mathrm{H})$. Data are in accordance with the one described in the literature. ${ }^{13}$

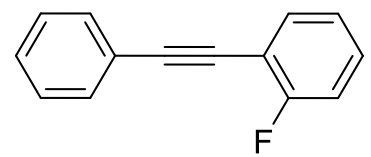

\section{1-Fluoro-2-(phenylethynyl)benzene (1s)}

Prepared according to general procedure A using 2fluoroiodobenzene on a $1.52 \mathrm{mmol}$ scale, the desired product (296 $\mathrm{mg}, 1.51 \mathrm{mmol}, 99 \%$ ) was isolated as a white solid after purification by flash column chromatography (hexanes). ${ }^{1} \mathrm{H}$ NMR $\left(400 \mathrm{MHz} \mathrm{CDCl}_{3}\right) \delta(\mathrm{ppm})=7.58-7.49$ (m, 3H), 7.38-7.27 (m, 4H), 7.15-7.07 (m, 2H); ${ }^{19} \mathrm{~F}$ NMR (376 MHz, $\left.\mathrm{CDCl}_{3}\right) \delta(\mathrm{ppm})=-109.9(\mathrm{~m}$, $1 F)$. Data are in accordance with the one described in the literature. ${ }^{14}$

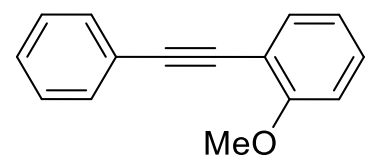

\section{1-Methoxy-2-(phenylethynyl)benzene (1t)}

Prepared according to general procedure A using 2-iodoanisole on a $1.21 \mathrm{mmol}$ scale, the desired product $(241 \mathrm{mg}, 1.16 \mathrm{mmol}, 96 \%)$ was isolated as a yellow oil after purification by automated flash purification (0-10\% EtOAc/hexanes). ${ }^{1} \mathrm{H}$ NMR (500 MHz, $\left.\mathrm{CDCl}_{3}\right) \delta(\mathrm{ppm})=7.60-7.53(\mathrm{~m}, 2 \mathrm{H}), 7.53-7.47$ (m,

\footnotetext{
12 Melzig, L.; Metzger, A.; Knochel, P., Chem. Eur. J. 2011, 17, 2948-2956.

${ }^{13}$ Kakusawa, N.; Yamaguchi, K.; Kurita, J., J. Organomet. Chem. 2005, 690, 2956-2966.

${ }^{14}$ Csékei, M.; Novák, Z.; Kotschy, A. Tetrahedron 2008, 64, 975-982.
} 
1H), 7.38-7.27 (m, 4H), 6.96-6.89 (m, 2H), $3.92(\mathrm{~s}, 3 \mathrm{H})$. Data are in accordance with the one described in the literature. ${ }^{11}$

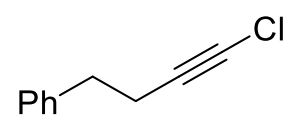

\section{(4-Bromobut-3-yn-1-yl)benzene (1u)}

To a 4-phenyl-1-butyne (0.35 mL, $2.49 \mathrm{mmol}, 1$ equiv.) solution in dry THF $(25 \mathrm{~mL}, 0.1 \mathrm{M})$ cooled to $-78^{\circ} \mathrm{C}$ is added dropwise $n$-butyllithium in THF $(1.5 \mathrm{~mL}$ of a 2.5 M solution in hexanes, $3.75 \mathrm{mmol}, 1.5$ equiv.). After the addition, the cooling bath is removed, and the reaction mixture is allowed to warm to room temperature. After 15 minutes, $N$-chlorosuccinimide $(505 \mathrm{mg}, 3.78 \mathrm{mmol}, 1.5$ equiv.) is added in one portion. After 24 hours, saturated $\mathrm{NH}_{4} \mathrm{Cl}$ is added and the reaction mixture is extracted with EtOAc (3x). Combined organic layers are washed with brine, dried over $\mathrm{Na}_{2} \mathrm{SO}_{4}$, filtered and evaporated in vacuo. The desired product (208 $\mathrm{mg}, 1.26 \mathrm{mmol}, 51 \%)$ is isolated as a colourless oil after purification by automated flash purification (hexanes). FT-IR $v\left(\mathrm{~cm}^{-1}\right)$ = 3028, 2949, 2928, 2862, 1605, 1497, 1454, 1076, 746, 698; ${ }^{1} \mathrm{H}$ NMR (500 MHz, $\left.\mathrm{CDCl}_{3}\right)$ $\delta(\mathrm{ppm})=7.33-7.28(\mathrm{~m}, 2 \mathrm{H}), 7.25-7.18(\mathrm{~m}, 3 \mathrm{H}), 2.82(\mathrm{t}, J=7.6 \mathrm{~Hz}, 2 \mathrm{H}), 2.46(\mathrm{t}, J=7.6$ $\mathrm{Hz}, 2 \mathrm{H}) ;{ }^{13} \mathrm{C} \mathrm{NMR}\left(126 \mathrm{MHz}, \mathrm{CDCl}_{3}\right) \delta(\mathrm{ppm})=140.4,128.6,128.5,126.5,69.1,58.1$, 34.9, 21.1; HRMS (APPI-TOF) m/z calcd for $\mathrm{C}_{10} \mathrm{H}_{9} \mathrm{Cl}\left[\mathrm{M}^{*}\right]^{+}$165.0421; found 165.0420.

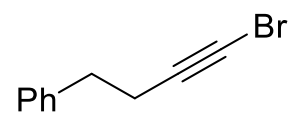

\section{(4-Chlorobut-3-yn-1-yl)benzene (1v)}

To a 4-phenyl-1-butyne ( $0.35 \mathrm{~mL}, 2.49 \mathrm{mmol}, 1$ equiv. $)$ solution in dry THF $(25 \mathrm{~mL}, 0.1 \mathrm{M})$ cooled to $-78^{\circ} \mathrm{C}$ is added dropwise $n$-butyllithium in THF $(1.5 \mathrm{~mL}$ of a $2.5 \mathrm{M}$ solution in hexanes, $3.75 \mathrm{mmol}, 1.5$ equiv.). After the addition, the cooling bath is removed, and the reaction mixture is allowed to warm to room temperature. After 15 minutes, $N$-bromosuccinimide (678 $\mathrm{mg}, 3.81 \mathrm{mmol}, 1.5$ equiv.) is added in one portion. After 18 hours, saturated $\mathrm{NH}_{4} \mathrm{Cl}$ is added and the reaction mixture is extracted with EtOAc (3x). Combined organic layers are washed with brine, dried over $\mathrm{Na}_{2} \mathrm{SO}_{4}$, filtered and evaporated in vacuo. The desired product $(336 \mathrm{mg}, 1.60 \mathrm{mmol}, 65 \%)$ is isolated as a colourless oil after purification by flash column chromatography (hexanes). ${ }^{1} \mathrm{H}$ NMR (400 
$\left.\mathrm{MHz}_{\mathrm{CDCl}}\right) \delta(\mathrm{ppm})=7.35-7.25(\mathrm{~m}, 2 \mathrm{H}), 7.26-7.16(\mathrm{~m}, 3 \mathrm{H}), 2.83(\mathrm{t}, J=7.6 \mathrm{~Hz}, 2 \mathrm{H})$, $2.49(\mathrm{t}, J=7.6 \mathrm{~Hz}, 2 \mathrm{H})$. Data are in accordance with the one described in the literature. ${ }^{15}$

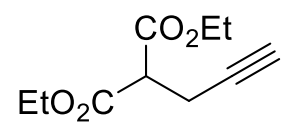

\section{Diethyl 2-(prop-2-yn-1-yl)malonate (1z)}

Sodium hydride (60\% in oil, $225 \mathrm{mg}, 5.62 \mathrm{mmol}, 1.1$ equiv.) is added by portions on diethylmalonate $(0.91 \mathrm{~mL}, 5.99 \mathrm{mmol}, 1.2$ equiv.) solution in dry THF (15 $\mathrm{mL}, 0.3 \mathrm{M})$ cooled à $0{ }^{\circ} \mathrm{C}$. After 15 minutes at $0{ }^{\circ} \mathrm{C}$, propargyl bromide $(0.56 \mathrm{~mL}$ of a 80 $\%$ solution in toluene, $5.03 \mathrm{mmol}, 1$ equiv.) is added dropwise. After 15 minutes, the ice bath is removed, and the reaction mixture is allowed to warm to room temperature for 6 hours. The reaction is partitioned in water and $\mathrm{Et}_{2} \mathrm{O}$. The aqueous layer is extracted with $\mathrm{Et}_{2} \mathrm{O}(2 \mathrm{x})$ and organic layers are combined, washed with $3 \mathrm{~N} \mathrm{HCl}$, saturated $\mathrm{NaHCO}_{3}$, and brine, dried over $\mathrm{Na}_{2} \mathrm{SO}_{4}$, filtered and evaporated in vacuo. The desired product $(272 \mathrm{mg}$, $1.37 \mathrm{mmol}, 27 \%$ ) was isolated as a colourless oil after purification by automated flash purification $\left(0-20 \% \mathrm{Et}_{2} \mathrm{O}\right.$ in hexanes). FT-IR $v\left(\mathrm{~cm}^{-1}\right)=3283,2986,1732,1369,1337$, 1271, 1236, 1176, 1155, 1034; ${ }^{1} \mathrm{H} \operatorname{NMR}\left(500 \mathrm{MHz}, \mathrm{CDCl}_{3}\right) \delta(\mathrm{ppm})=4.23(\mathrm{qd}, J=7.1$, $2.5 \mathrm{~Hz}, 4 \mathrm{H}), 3.57(\mathrm{t}, J=7.7 \mathrm{~Hz}, 1 \mathrm{H}), 2.78(\mathrm{dd}, J=7.7,2.7 \mathrm{~Hz}, 2 \mathrm{H}), 2.02(\mathrm{t}, J=2.7 \mathrm{~Hz}$, $1 \mathrm{H}), 1.28(\mathrm{t}, J=7.1 \mathrm{~Hz}, 6 \mathrm{H}) ;{ }^{13} \mathrm{C} \mathrm{NMR}\left(126 \mathrm{MHz} \mathrm{CDCl}_{3}\right) \delta(\mathrm{ppm})=168.0,80.1,70.5$, 61.9, 51.3, 18.5, 14.2; HRMS (ESI-TOF) $\mathrm{m} / \mathrm{z}$ calcd for $\mathrm{C}_{10} \mathrm{H}_{15} \mathrm{O}_{4}[\mathrm{M}+\mathrm{H}]^{+}$199.0965; found 199.0965.

\section{Synthesis of gold catalyst dimer $\left[\{\mathrm{Au}(\mathrm{JohnPhos})\}_{2}(\mu \mathrm{OH})\right] \mathrm{OTf}$}

Preparation of the catalyst is inspired by procedure from Ramon et al. ${ }^{16}$ : Silver trifluorométhanesulfonate (199 mL, $0.78 \mathrm{mmol}, 1.07$ equiv.) is added on (JohnPhos) $\mathrm{AuCl}$ (386 mg, $0.73 \mathrm{mmol}, 1$ equiv.) solution in dry $\mathrm{CH}_{2} \mathrm{Cl}_{2}(3.6 \mathrm{~mL}, 0.2 \mathrm{M})$. After 15 minutes, the reaction mixture is filtered on Celite ${ }^{\circledR}$ in a Pasteur pipette. Water is added to the filtrate and the combined phases are vigorously shaken in an extraction funnel. The organic layer is dried over $\mathrm{Na}_{2} \mathrm{SO}_{4}$, filtered and evaporated in vacuo. The desired product (392 $\mathrm{mg}, 0.34$ mmol, 93\%) was isolated as a white solid without further purification. FT-IR $v\left(\mathrm{~cm}^{-1}\right)=$

\footnotetext{
15 Okutani, M.; Mori, Y., J. Org. Chem. 2009, 74, 442-444.

${ }^{16}$ Ramon, R.S.; Gaillard, S.; Poater A.; Cavallo, L.; Slawin, A.M.Z.; Nolan, S.P., Chem. Eur. J. 2011, 17, 1238-1246.
} 
2966, 2959, 2249, 1474, 1464, 1393, 1271, 1263, 1151, 1032; ${ }^{1} \mathrm{H}$ NMR (500 MHz, $\left.\mathrm{CDCl}_{3}\right)$ $\delta(\mathrm{ppm})=7.90-7.83(\mathrm{~m}, 2 \mathrm{H}), 7.61-7.54(\mathrm{~m}, 6 \mathrm{H}), 7.47(\mathrm{t}, J=7.7 \mathrm{~Hz}, 4 \mathrm{H}), 7.34-7.28(\mathrm{~m}$, $2 \mathrm{H}), 7.22-7.16(\mathrm{~m}, 4 \mathrm{H}), 1.40(\mathrm{~s}, 18 \mathrm{H}), 1.37$ (s, 18H), -0.25 (m, 1H); ${ }^{13} \mathrm{C} \mathrm{NMR}(126 \mathrm{MHz}$, $\left.\mathrm{CDCl}_{3}\right) \delta(\mathrm{ppm})=148.9\left(\mathrm{~d}, J_{C-P}=12.5 \mathrm{~Hz}\right), 143.6\left(\mathrm{~d}, J_{C-P}=6.5 \mathrm{~Hz}\right), 133.1\left(\mathrm{~d}, J_{C-P}=3.7\right.$ $\mathrm{Hz}), 131.3,130.2,128.4,127.6\left(\mathrm{~d}, J_{C-P}=7.7 \mathrm{~Hz}\right), 127.2,124.8\left(\mathrm{~d}, J_{C-P}=49.9 \mathrm{~Hz}\right), 37.7$ $\left(\mathrm{d}, J_{C-P}=27.8 \mathrm{~Hz}\right), 30.7\left(\mathrm{~d}, J_{C-P}=6.3 \mathrm{~Hz}\right) ;{ }^{19} \mathrm{~F} \mathrm{NMR}\left(470 \mathrm{MHz}, \mathrm{CDCl}_{3}\right) \delta(\mathrm{ppm})=-77.9$ (s, 3F); ${ }^{31} \mathrm{P} \mathrm{NMR}\left(202 \mathrm{MHz}, \mathrm{CDCl}_{3}\right) \delta(\mathrm{ppm})=57.2(\mathrm{~s}, 2 \mathrm{P}) ; \mathrm{HRMS}($ ESI-TOF) $\mathrm{m} / \mathrm{z}$ calcd for $\mathrm{C}_{40} \mathrm{H}_{55} \mathrm{Au}_{2} \mathrm{P}_{2} \mathrm{O}$ [M-OTf] ${ }^{+}$1007.3054; found 1007.3013.

\section{General procedures for the hydrofluorination of alkynes}

In a $1.5 \mathrm{~mL}$ polypropylene Eppendorf, a solution of alkyne in toluene $(0.3 \mathrm{M})$ was added in $48 \%$ aqueous HF ( 2 equiv.). Then, [ $\left.\{\mathrm{Au}(\mathrm{JohnPhos})\}_{2}(\mu \mathrm{OH})\right] \mathrm{OTf}(3 \mathrm{~mol} \%)$ was added and the reaction was stirred for 18 hours at $40^{\circ} \mathrm{C}$. The reaction was quenched with saturated solution of $\mathrm{NaHCO}_{3}$ and extracted with EtOAc (3x). Combined organic layers were washed with water $(2 \mathrm{x})$ and brine, dried over $\mathrm{Na}_{2} \mathrm{SO}_{4}$, filtered and concentrated in vacuo. The desired monofluoroalkene was isolated after purification by flash column chromatography. Compounds $2 \mathbf{a},{ }^{17} \mathbf{2 g},{ }^{18} \mathbf{2 i},{ }^{19} \mathbf{2} \mathbf{x}^{20}$ and $2 \mathbf{y}^{21}$ were identified on ${ }^{19} \mathrm{~F}$ NMR of the crude mixtures from know spectral data in the literature. Spectra used to calculate NMR yields are furnished in section 5 .

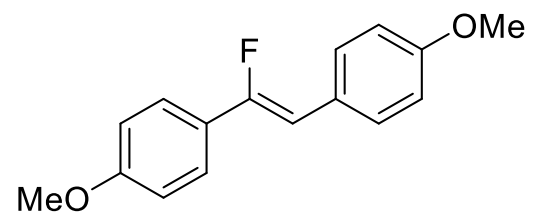

\section{(Z)-4,4'-(1-fluoroethene-1,2-diyl)bis (methoxybenzene) (2b)}

Prepared according to the general procedure on a 0.128 mmol scale, the desired product $(23.2 \mathrm{mg}, 0.090 \mathrm{mmol}$, 70\%) was isolated as a white solid after purification by automated flash purification (5$15 \%$ EtOAc/hexanes). ${ }^{1} \mathrm{H}$ NMR (500 MHz, $\left.\mathrm{CDCl}_{3}\right) \delta(\mathrm{ppm})=7.61-7.49(\mathrm{~m}, 4 \mathrm{H}), 7.01-$ $6.84(\mathrm{~m}, 4 \mathrm{H}), 6.12(\mathrm{~d}, J=40.1 \mathrm{~Hz}, 1 \mathrm{H}), 3.84$ (s, 3H), 3.83 (s, 3H); ${ }^{19} \mathrm{~F}$ NMR $(470 \mathrm{MHz}$,

\footnotetext{
${ }^{17}$ Yoshide, M.; Komata, A.; Hara, S., Tetrahedron 2006, 62, 8636-8645.

${ }^{18}$ Sashuk, V.; Ignatowska, J.; Grela, K., J. Org. Chem. 2004, 69, 7748-7751.

${ }^{19}$ Wong, O.A.; Shi, Y., J. Org. Chem. 2009, 74, 8377-8380.

${ }^{20}$ Nguyen, T.-H.; Abarati, M.; Guilloteau, D.; Mavel, S.; Emond, P., Tetrahedron 2011, 67, 3434-3439.

${ }^{21}$ Schlosser, M.; Brügger, N.; Schmidt, W.; Amrhein, N., Tetrahedron 2004, 60, 7731-7742.
} 
$\left.\mathrm{CDCl}_{3}\right) \delta(\mathrm{ppm})=-116.2(\mathrm{~d}, J=41.1 \mathrm{~Hz}, 1 \mathrm{~F})$. Data are in accordance with the one described in the literature. ${ }^{2}$

$1 \mathrm{mmol}$ scale procedure: In a $15 \mathrm{~mL}$ polypropylene vial, a solution of 1,2-bis(4methoxyphenyl)ethyne (240 mg, $1.00 \mathrm{mmol}, 1$ equiv.) in toluene $(3.5 \mathrm{~mL}, 0.3 \mathrm{M})$ was added in $48 \%$ aqueous $\mathrm{HF}$ (84 $\mathrm{mg}, 2.03 \mathrm{mmol}, 2$ equiv.). Then, $\left[\{\mathrm{Au}(\mathrm{JohnPhos})\}_{2}(\mu \mathrm{OH})\right] \mathrm{OTf}(35 \mathrm{mg}, 30 \mu \mathrm{mol}, 3 \mathrm{~mol} \%)$ was added and the reaction was stirred for 18 hours at $40{ }^{\circ} \mathrm{C}$. The reaction was cooled to $0{ }^{\circ} \mathrm{C}$, quenched with saturated solution of $\mathrm{NaHCO}_{3}$ and extracted with EtOAc (3x). Combined organic layers were washed with water $(2 \mathrm{x})$ and brine, dried over $\mathrm{Na}_{2} \mathrm{SO}_{4}$, filtered and concentrated in vacuo. The desired product (157 mg, $0.60 \mathrm{mmol}, 60 \%$ ) was isolated as a white solid after purification by flash column chromatography (10\% $\mathrm{Et}_{2} \mathrm{O} /$ hexanes). Spectral data are in accordance with the one described above.

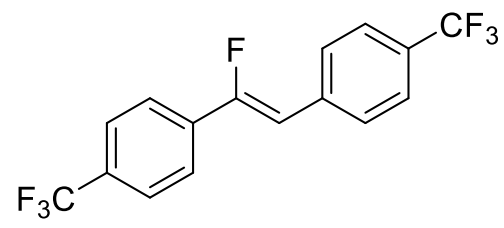

\section{(Z)-4,4'-(1-fluoroethene-1,2-diyl)bis ((trifluoromethyl)benzene) (2c)}

Prepared according to the general procedure on a 0.095 mmol scale, the desired product $(26.7 \mathrm{mg}, 0.080 \mathrm{mmol}$, 84\%) was isolated as a white solid after purification by automated flash purification (hexanes). Mp: $105.8-108.3{ }^{\circ} \mathrm{C}$; FT-IR $v\left(\mathrm{~cm}^{-1}\right)=2982,2924,1657,1618,1418,1317$, $1161,1107,1067,1011 ;{ }^{1} \mathrm{H}$ NMR $\left(400 \mathrm{MHz}, \mathrm{CDCl}_{3}\right) \delta(\mathrm{ppm})=7.81-7.72(\mathrm{~m}, 4 \mathrm{H}), 7.73-$ $7.60(\mathrm{~m}, 4 \mathrm{H}), 6.44(\mathrm{~d}, J=38.3 \mathrm{~Hz}, 1 \mathrm{H}) ;{ }^{13} \mathrm{C} \mathrm{NMR}\left(126 \mathrm{MHz}, \mathrm{CDCl}_{3}\right) \delta(\mathrm{ppm})=157.2(\mathrm{~d}$, $\left.J_{C-F}=261.3 \mathrm{~Hz}\right), 136.6-136.4(\mathrm{~m}), 135.6\left(\mathrm{dq}, J_{C-F}=28.3,1.2 \mathrm{~Hz}\right), 131.3\left(\mathrm{q}, J_{C-F}=32.7\right.$ $\mathrm{Hz}), 129.6\left(\mathrm{qd}, J_{C-F}=32.6,2.8 \mathrm{~Hz}\right), 129.3\left(\mathrm{~d}, J_{C-F}=8.2 \mathrm{~Hz}\right), 125.7\left(\mathrm{qd}, J_{C-F}=3.9,2.0\right.$ $\mathrm{Hz}), 125.6\left(\mathrm{q}, J_{C-F}=3.8 \mathrm{~Hz}\right), 124.7\left(\mathrm{~d}, J_{C-F}=7.6 \mathrm{~Hz}\right), 124.1\left(\mathrm{q}, J_{C-F}=271.9 \mathrm{~Hz}\right), 128.8$ $\left(\mathrm{q}, J_{C-F}=272.1 \mathrm{~Hz}\right), 106.7\left(\mathrm{~d}, J_{C-F}=9.8 \mathrm{~Hz}\right) ;{ }^{19} \mathrm{~F}$ NMR $\left(376 \mathrm{MHz}, \mathrm{CDCl}_{3}\right) \delta(\mathrm{ppm})=-$ 62.7 (s, 3F), -62.9 (s, 3F), -112.1 (d, $J=38.3 \mathrm{~Hz}, 1 \mathrm{~F})$; HRMS (APPI-TOF) $\mathrm{m} / \mathrm{z}$ calcd for $\mathrm{C}_{16} \mathrm{H}_{10} \mathrm{FO}_{7}[\mathrm{M}+\mathrm{H}]^{+}$335.0665; found 335.0649. 
<smiles>FC(=Cc1ccc(Cl)cc1)c1ccc(Cl)cc1</smiles>

\section{(Z)-4,4'-(1-Fluoroethene-1,2-diyl)bis(chlorobenzene)} (2d)

Prepared according to the general procedure on a $0.170 \mathrm{mmol}$ scale, the desired product (contaminated with $5 \%$ starting material) (32.9 mg, $0.123 \mathrm{mmol}, 67 \%$ corrected yield) was isolated as a white solid after purification by flash column chromatography (hexanes). ${ }^{1} \mathrm{H}$ NMR $\left(500 \mathrm{MHz}, \mathrm{CDCl}_{3}\right) \delta$ $(\mathrm{ppm})=7.59-7.52(\mathrm{~m}, 4 \mathrm{H}), 7.42-7.35(\mathrm{~m}, 2 \mathrm{H}), 7.37-7.31(\mathrm{~m}, 2 \mathrm{H}), 6.24(\mathrm{~d}, J=38.9 \mathrm{~Hz}$, $1 \mathrm{H}) ;{ }^{19} \mathrm{~F}$ NMR $\left(470 \mathrm{MHz}, \mathrm{CDCl}_{3}\right) \delta(\mathrm{ppm})=-113.7(\mathrm{~d}, J=38.2 \mathrm{~Hz}, 1 \mathrm{~F})$. Data are in accordance with the one described in the literature. ${ }^{2}$<smiles>COc1cccc(/C=C(\F)c2cccc(OC)c2)c1</smiles>

\section{(Z)-3,3'-(1-Fluoroethene-1,2- diyl)bis(methoxybenzene) (2e)}

Prepared according to the general procedure on a 0.191 mmol scale, the desired product $(36.0 \mathrm{mg}, 0.140 \mathrm{mmol}, 73 \%)$ was isolated as a colourless oil after purification by flash column chromatography (5\% Et $2 \mathrm{O} /$ hexanes). ${ }^{1} \mathrm{H}$ NMR (500 $\left.\mathrm{MHz}, \mathrm{CDCl}_{3}\right) \delta(\mathrm{ppm})=7.34-7.26(\mathrm{~m}, 2 \mathrm{H}), 7.25-7.18(\mathrm{~m}, 3 \mathrm{H}), 7.17-7.15(\mathrm{~m}, 1 \mathrm{H}), 6.86$ (dddd, $J=37.2,8.2,2.7,1.0 \mathrm{~Hz}, 2 \mathrm{H}), 6.28$ (d, $J=39.3 \mathrm{~Hz}, 1 \mathrm{H}), 3.84$ (s, 3H), 3.83 (s, 3H); ${ }^{19} \mathrm{~F} \mathrm{NMR}\left(470 \mathrm{MHz}, \mathrm{CDCl}_{3}\right) \delta(\mathrm{ppm})=-112.9(\mathrm{~d}, J=38.8 \mathrm{~Hz}, 1 \mathrm{~F})$. Data are in accordance with the one described in the literature. ${ }^{2}$

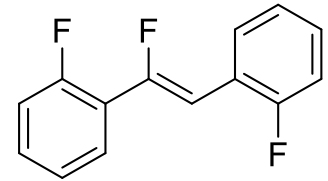

\section{(Z)-2,2'-(1-Fluoroethene-1,2-diyl)bis(fluorobenzene) (2f)}

Prepared according to the general procedure on a $0.173 \mathrm{mmol}$ scale, the desired product (16.7 $\mathrm{mg}, 0.071 \mathrm{mmol}, 41 \%)$ was isolated as a colourless oil after purification by flash column chromatography (hexanes). FT-IR $v\left(\mathrm{~cm}^{-}\right.$ $\left.{ }^{1}\right)=2982,2924,1578,1495,1483,1456,1229,1034,1016,749 ;{ }^{1} \mathrm{H}$ NMR $(400 \mathrm{MHz}$, $\left.\mathrm{CDCl}_{3}\right) \delta(\mathrm{ppm})=7.58-7.49(\mathrm{~m}, 2 \mathrm{H}), 7.37-7.27(\mathrm{~m}, 2 \mathrm{H}), 7.16-7.05(\mathrm{~m}, 4 \mathrm{H}) ;{ }^{13} \mathrm{C} \mathrm{NMR}(101$ $\left.\mathrm{MHz} \mathrm{CDCl}_{3}\right) \delta(\mathrm{ppm})=159.8\left(\mathrm{~d}, J_{C-F}=250.3 \mathrm{~Hz}\right), 159.5\left(\mathrm{dd}, J_{C-F}=252.8,5.4 \mathrm{~Hz}\right), 153.0$ $\left(\mathrm{ddd}, J_{C-F}=258.1,5.8,2.4 \mathrm{~Hz}\right), 127.4\left(\mathrm{dd}, J_{C-F}=9.0,2.0 \mathrm{~Hz}\right), 124.3\left(\mathrm{dd}, J_{C-F}=3.8,1.5\right.$ $\mathrm{Hz}), 124.2\left(\mathrm{~d}, J_{C-F}=3.6 \mathrm{~Hz}\right), 121.5\left(\mathrm{dd}, J_{C-F}=11.8,2.8 \mathrm{~Hz}\right), 120.8\left(\mathrm{dd}, J_{C-F}=29.5,10.7\right.$ $\mathrm{Hz}), 116.4\left(\mathrm{dd}, J_{C-F}=22.8,2.5 \mathrm{~Hz}\right), 115.3\left(\mathrm{~d}, J_{C-F}=22.2 \mathrm{~Hz}\right), 102.8\left(\mathrm{dt}, J_{C-F}=14.0,8.0\right.$ $\mathrm{Hz}) ;{ }^{19} \mathrm{~F} \mathrm{NMR}\left(470 \mathrm{MHz}, \mathrm{CDCl}_{3}\right) \delta(\mathrm{ppm})=-108.8(\mathrm{ddd}, J=41.3,9.7,5.1 \mathrm{~Hz}, 1 \mathrm{~F}),-111.5$ 
(ddt, $J=16.3,11.2,5.4 \mathrm{~Hz}, 1 \mathrm{~F}$ ), -116.0 (dt, $J=12.7,5.7 \mathrm{~Hz}, 1 \mathrm{~F}$ ); HRMS (APPI-TOF) $\mathrm{m} / \mathrm{z}$ calcd for $\mathrm{C}_{14} \mathrm{H}_{10} \mathrm{~F}_{3}[\mathrm{M}+\mathrm{H}]^{+}$235.0729; found 235.0711.

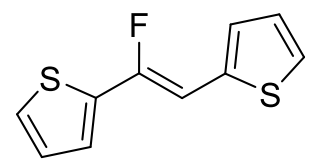

\section{(Z)-2,2'-(1-Fluoroethene-1,2-diyl)dithiophene (2h)}

Prepared according to the general procedure on a $0.183 \mathrm{mmol} \mathrm{scale}$, the desired product (30.8 $\mathrm{mg}, 0.146 \mathrm{mmol}, 80 \%)$ was isolated as a yellow solid after purification by flash column chromatography (hexanes). ${ }^{1} \mathrm{H}$ NMR (500 $\left.\mathrm{MHz}, \mathrm{CDCl}_{3}\right) \delta(\mathrm{ppm})=7.36-7.28(\mathrm{~m}, 3 \mathrm{H}), 7.20-7.16(\mathrm{~m}, 1 \mathrm{H}), 7.09-7.01(\mathrm{~m}, 2 \mathrm{H}), 6.48(\mathrm{~d}$, $J=37.7 \mathrm{~Hz}, 1 \mathrm{H}), 3.84(\mathrm{~s}, 3 \mathrm{H}), 3.83(\mathrm{~s}, 3 \mathrm{H}) ;{ }^{19} \mathrm{~F}$ NMR $\left(470 \mathrm{MHz}, \mathrm{CDCl}_{3}\right) \delta(\mathrm{ppm})=-$ $105.7(\mathrm{~d}, J=38.0 \mathrm{~Hz}, 1 \mathrm{~F})$. Data are in accordance with the one described in the literature. ${ }^{2}$<smiles>CCCC=CC(F)=C[18OH]</smiles>

\section{(Z)-6-Fluorododec-6-ene (2j)}

Prepared according to the general procedure on a $0.262 \mathrm{mmol}$ scale, the desired product ( $25.4 \mathrm{mg}, 0.136 \mathrm{mmol}, 52 \%)$ was isolated as a colourless oil after purification by flash column chromatography (hexanes). FT-IR $v\left(\mathrm{~cm}^{-1}\right)=2961,2930$, $2870,2166,2023 ;{ }^{1} \mathrm{H}$ NMR $\left(500 \mathrm{MHz}, \mathrm{CDCl}_{3}\right) \delta(\mathrm{ppm})=4.45(\mathrm{dt}, J=38.3,7.4 \mathrm{~Hz}, 1 \mathrm{H})$, $2.12(\mathrm{dt}, J=17.7,7.5 \mathrm{~Hz}, 2 \mathrm{H}), 2.04(\mathrm{dt}, J=7.8,7.4 \mathrm{~Hz}, 2 \mathrm{H}), 1.48(\mathrm{tt}, J=7.3,7.2 \mathrm{~Hz}, 2 \mathrm{H})$, 1.38-1.23 (m, 10H), 0.93-0.85 (m, 6H); ${ }^{13} \mathrm{C}$ NMR (101 MHz, $\left.\mathrm{CDCl}_{3}\right) \delta(\mathrm{ppm})=159.6(\mathrm{~d}$, $\left.J_{C-F}=252.3 \mathrm{~Hz}\right), 104.9\left(\mathrm{~d}, J_{C-F}=16.2 \mathrm{~Hz}\right), 32.0\left(\mathrm{~d}, J_{C-F}=27.9 \mathrm{~Hz}\right), 31.3\left(\mathrm{~d}, J_{C-F}=26.6\right.$ $\mathrm{Hz}), 29.3\left(\mathrm{~d}, J_{C-F}=1.7 \mathrm{~Hz}\right), 26.0\left(\mathrm{~d}, J_{C-F}=1.3 \mathrm{~Hz}\right), 23.5,23.4,22.5,22.4,14.1,14.0 ;{ }^{19} \mathrm{~F}$ $\operatorname{NMR}\left(470 \mathrm{MHz}, \mathrm{CDCl}_{3}\right) \delta(\mathrm{ppm})=-110.3(\mathrm{dt}, J=36.8,17.8 \mathrm{~Hz}, 1 \mathrm{~F}) ; \mathrm{GC}-\mathrm{MS}$ (EI) m/z calcd for $\mathrm{C}_{12} \mathrm{H}_{23} \mathrm{~F}[\mathrm{M}]^{+}$186.18; found 186.40. Under all other conditions tested for MS analysis (HRMS-ESI(+). HRMS-ESI(-), HRMS-APPI and GC-MS-CI), we never could detect any significant ions.

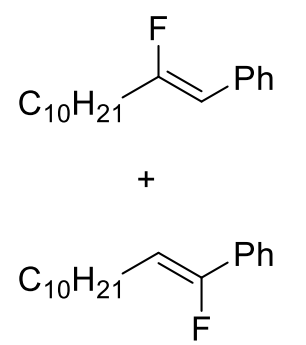

\section{(Z)-(2-fluorododec-1-en-1-yl)benzene + (Z)-(1-fluorododec-1-en-1- yl)benzene (69:31) $\left(2 \mathrm{k}+2 \mathrm{k}^{\prime}\right)$}

Prepared according to the general procedure on a $0.171 \mathrm{mmol}$ scale, the desired product (mixture of regioisomers 69:31, $32.9 \mathrm{mg}, 0.125 \mathrm{mmol}$, $73 \%$ ) was isolated as a colourless oil after purification by automated flash purification (hexanes). FT-IR $v\left(\mathrm{~cm}^{-1}\right)=2953,2924,2854,1690,1495,1466,1448$, 
1053, 1034, 1016; ${ }^{1} \mathrm{H}$ NMR (500 MHz, $\left.\mathrm{CDCl}_{3}\right) \delta(\mathrm{ppm})=7.51-7.44$ (m, 2H, 2k + 2k'), 7.37-7.26 (m, 2.29H, 2k + 2k'), 7.21-7.16 (m, 0.66H, 2k) 5.45 (d, J=39.5 Hz, 0.67H, 2k), 5.39 (dt, $J=37.5,7.6 \mathrm{~Hz}, 0.33 \mathrm{H}, \mathbf{2 k}$ '), 2.35-2.24 (m, 2H, 2k + 2k'), 1.63-1.55 (m, 1.38H, 2k), 1.48-1.41 (m, 0.62H, 2k'), 1.40-1.20 (m, 14H, 2k + 2k'), 0.88 (t, J = 6.9 Hz, 3H, 2k + 2k'); ${ }^{13} \mathrm{C}$ NMR $\left(126 \mathrm{MHz}, \mathrm{CDCl}_{3}\right) \delta(\mathrm{ppm})=161.3\left(\mathrm{~d}, J_{C-F}=266.9 \mathrm{~Hz}\right), 156.6\left(\mathrm{~d}, J_{C-F}\right.$ $=245.5 \mathrm{~Hz}), 134.0\left(\mathrm{~d}, J_{C-F}=2.4 \mathrm{~Hz}\right), 132.9\left(\mathrm{~d}, J_{C-F}=29.5 \mathrm{~Hz}\right), 128.37,128.36,128.24$, $128.23\left(\mathrm{~d}, J_{C-F}=7.2 \mathrm{~Hz}\right), 126.6\left(\mathrm{~d}, J_{C-F}=2.3 \mathrm{~Hz}\right), 123.8\left(\mathrm{~d}, J_{C-F}=6.8 \mathrm{~Hz}\right), 106.4\left(\mathrm{~d}, J_{C-F}\right.$ $=18.0 \mathrm{~Hz}), 105.6\left(\mathrm{~d}, J_{C-F}=8.9 \mathrm{~Hz}\right), 33.1\left(\mathrm{~d}, J_{C-F}=26.4 \mathrm{~Hz}\right), 31.93,31.92,29.64,29.61$, 29.55, 29.48, $29.45\left(\mathrm{~d}, J_{C-F}=1.7 \mathrm{~Hz}\right), 29.38,29.37,29.34,29.30,29.0,26.4\left(\mathrm{~d}, J_{C-F}=1.0\right.$ $\mathrm{Hz}), 24.1\left(\mathrm{~d}, J_{C-F}=4.9 \mathrm{~Hz}\right), 22.7,14.1 ;{ }^{19} \mathrm{~F} \mathrm{NMR}\left(470 \mathrm{MHz}, \mathrm{CDCl}_{3}\right) \delta(\mathrm{ppm})=-100.6(\mathrm{dt}$, $J=39.4,18.2 \mathrm{~Hz}, 0.69 \mathrm{~F}, 2 \mathbf{k}),-121.4$ (d, $\left.J=37.4 \mathrm{~Hz}, 0.31 \mathrm{~F}, 2 \mathbf{k}^{\prime}\right)$; HRMS (APPI-TOF) m/z calcd for $\mathrm{C}_{18} \mathrm{H}_{27} \mathrm{~F}\left[\mathrm{M}^{*}\right]^{+}$262.2091; found 262.2088. The major regioisomer was identified from the expected coupling pattern in ${ }^{19} \mathrm{~F}$ NMR (doublet of triplet). The $\mathrm{Z}$ stereoisomers are established by the coupling constants between vinylic fluorine and proton (39.4 and $37.4 \mathrm{~Hz}=$ trans coupling). ${ }^{22}$

\section{F $\quad(Z)-5-F l u o r o-6-p h e n y l h e x-5-e n-1-y l$ acetate (2l)}

Prepared according to the general procedure on a $0.159 \mathrm{mmol}$ scale, the major regioisomer $(19.3 \mathrm{mg}, 0.082 \mathrm{mmol}, 51 \%)$ was isolated as a colourless oil after purification by flash column chromatography $\left(5 \%\right.$ EtOAc/hexanes). FT-IR $v\left(\mathrm{~cm}^{-1}\right)=$ 2955, 1736, 1690, 1495, 1450, 1387, 1366, 1236, 1149, 1041; ${ }^{1} \mathrm{H}$ NMR (500 MHz, $\left.\mathrm{CDCl}_{3}\right)$ $\delta(\mathrm{ppm})=7.49-7.43(\mathrm{~m}, 2 \mathrm{H}), 7.35-7.28(\mathrm{~m}, 2 \mathrm{H}), 7.24-7.17(\mathrm{~m}, 1 \mathrm{H}), 5.48(\mathrm{~d}, J=39.4 \mathrm{~Hz}$, $1 \mathrm{H}), 4.10(\mathrm{t}, J=6.3 \mathrm{~Hz}, 2 \mathrm{H}), 2.37(\mathrm{dt}, J=18.3,7.1 \mathrm{~Hz}, 2 \mathrm{H}), 2.06$ (s, 3H), 1.78-1.63 (m, $4 \mathrm{H}) ;{ }^{13} \mathrm{C} \mathrm{NMR}\left(101 \mathrm{MHz}, \mathrm{CDCl}_{3}\right) \delta(\mathrm{ppm})=171.2,160.4\left(\mathrm{~d}, J_{C-F}=266.6 \mathrm{~Hz}\right), 133.7(\mathrm{~d}$, $\left.J_{C-F}=2.6 \mathrm{~Hz}\right), 128.4,128.3\left(\mathrm{~d}, J_{C-F}=7.3 \mathrm{~Hz}\right), 126.8\left(\mathrm{~d}, J_{C-F}=2.3 \mathrm{~Hz}\right), 106.2\left(\mathrm{~d}, J_{C-F}=\right.$ $8.6 \mathrm{~Hz}), 64.1,32.7\left(\mathrm{~d}, J_{C-F}=26.8 \mathrm{~Hz}\right), 27.8,22.8,21.0 ;{ }^{19} \mathrm{~F} \mathrm{NMR}\left(470 \mathrm{MHz}, \mathrm{CDCl}_{3}\right) \delta$ $(\mathrm{ppm})=-101.6(\mathrm{dt}, J=39.6,18.4 \mathrm{~Hz}, 1 \mathrm{~F}) ; \mathrm{HRMS}\left(\right.$ APPI-TOF) $\mathrm{m} / \mathrm{z}$ calcd for $\mathrm{C}_{14} \mathrm{H}_{21} \mathrm{FNO}_{2}$ $\left[\mathrm{M}+\mathrm{NH}_{4}\right]^{+}$256.1610; found 256.1604. The regioisomer is identified from the coupling

\footnotetext{
${ }^{22}$ Dolbier, W.R., Jr. Guide to Fluorine NMR for Organic Chemists, Wiley, 2009.
} 
pattern in ${ }^{19} \mathrm{~F}$ NMR (doublet of triplet). The $\mathrm{Z}$ stereoisomer is confirmed by coupling constant between vinylic fluorine and proton $(39.6 \mathrm{~Hz}=$ trans coupling $){ }^{22}$

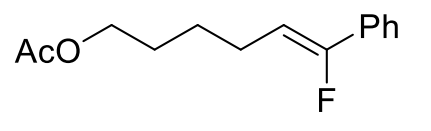

\section{(Z)-6-Fluoro-6-phenylhex-5-en-1-yl acetate (21')}

Prepared according to the general procedure on a $0.159 \mathrm{mmol}$ scale, the minor regioisomer $(13.5 \mathrm{mg}, 0.057 \mathrm{mmol}, 36 \%$ ) was isolated as a colourless oil after purification by flash column chromatography (5\% EtOAc/hexanes). FT-IR $v\left(\mathrm{~cm}^{-1}\right)=$ 2949, 2862, 1736, 1678, 1497, 1448, 1366, 1279, 1238, 1036; ${ }^{1} \mathrm{H}$ NMR (500 MHz, $\left.\mathrm{CDCl}_{3}\right)$ $\delta(\mathrm{ppm})=7.54-7.46(\mathrm{~m}, 2 \mathrm{H}), 7.39-7.27(\mathrm{~m}, 3 \mathrm{H}), 5.39(\mathrm{dt}, J=37.2,7.6 \mathrm{~Hz}, 1 \mathrm{H}), 4.10(\mathrm{t}, J$ $=6.6 \mathrm{~Hz}, 2 \mathrm{H}), 2.37-2.28(\mathrm{~m}, 2 \mathrm{H}), 2.06(\mathrm{~s}, 3 \mathrm{H}), 1.74-1.67(\mathrm{~m}, 2 \mathrm{H}), 1.57-1.50(\mathrm{~m}, 2 \mathrm{H}) ;{ }^{13} \mathrm{C}$ $\operatorname{NMR}\left(101 \mathrm{MHz}, \mathrm{CDCl}_{3}\right) \delta(\mathrm{ppm})=171.2,157.0\left(\mathrm{~d}, J_{C-F}=246.1 \mathrm{~Hz}\right), 132.6\left(\mathrm{~d}, J_{C-F}=29.1\right.$ $\mathrm{Hz}), 128.44,128.41\left(\mathrm{~d}, J_{C-F}=2.0 \mathrm{~Hz}\right), 123.9\left(\mathrm{~d}, J_{C-F}=7.1 \mathrm{~Hz}\right), 105.5\left(\mathrm{~d}, J_{C-F}=17.7 \mathrm{~Hz}\right)$, 64.3, 28.2, $25.8\left(\mathrm{~d}, J_{C-F}=1.7 \mathrm{~Hz}\right), 23.7\left(\mathrm{~d}, J_{C-F}=5.1 \mathrm{~Hz}\right), 21.0 ;{ }^{19} \mathrm{~F}$ NMR $(470 \mathrm{MHz}$, $\left.\mathrm{CDCl}_{3}\right) \delta(\mathrm{ppm})=-120.7(\mathrm{~d}, J=38.0 \mathrm{~Hz}, 1 \mathrm{~F}) ; \mathrm{HRMS}($ APPI-TOF) $\mathrm{m} / \mathrm{z}$ calcd for $\mathrm{C}_{14} \mathrm{H}_{17} \mathrm{FO}_{2}\left[\mathrm{M}^{*}\right]^{+}$237.1241; found 237.1244.

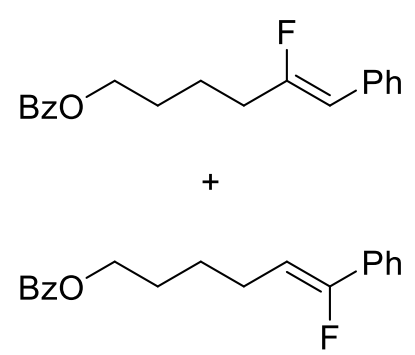

(Z)-5-fluoro-6-phenylhex-5-en-1-yl benzoate $+(Z)$-6-fluoro6-phenylhex-5-en-1-yl benzoate $(62: 38)(2 \mathrm{~m}+2 \mathrm{~m}$ ')

Prepared according to the general procedure on a $0.187 \mathrm{mmol}$ scale, the desired product (mixture of regioisomers 62:38, 39.4 $\mathrm{mg}, 0.132 \mathrm{mmol}, 71 \%$ ) was isolated as a colourless oil after purification by flash column chromatography $(5 \%$

$\mathrm{Et}_{2} \mathrm{O} /$ hexanes$)$. FT-IR $v\left(\mathrm{~cm}^{-1}\right)=2953,1717,1691,1603,1450,1315,1271,1176,1115$, 1070; ${ }^{1} \mathrm{H}$ NMR $\left(500 \mathrm{MHz}, \mathrm{CDCl}_{3}\right) \delta(\mathrm{ppm})=8.08-8.02(\mathrm{~m}, 2 \mathrm{H}, \mathbf{2 m}+\mathbf{2 m}$ '), 7.58-7.52 (m, 1H, 2m + 2m'), 7.52-7.41 (m, 4H, 2m + 2m'), 7.38-7.27 (m, 2.41H, 2m + 2m'), 7.22-7.17 $(\mathrm{m}, 0.61 \mathrm{H}, \mathbf{2 m}), 5.50$ (d, $J=39.3 \mathrm{~Hz}, 0.38 \mathrm{H}, \mathbf{2 m}$ '), 5.41 (dt, $J=37.2,7.7 \mathrm{~Hz}, 0.62 \mathrm{H}, 2 \mathbf{m})$, 4.36 (m, 2H, 2m + 2m'), 2.46-2.34 (m, 2H, $\mathbf{2 m}+\mathbf{2 m}$ '), 1.91-1.74 (m, 3.36H, $\mathbf{2 m}+\mathbf{2 m}$ '), 1.67-1.59 (m, 0.64H, 2m); $\left.{ }^{13} \mathrm{C} \mathrm{NMR} \mathrm{(126} \mathrm{MHz,} \mathrm{CDCl}_{3}\right) \delta(\mathrm{ppm})=166.7,166.6,160.4(\mathrm{~d}$, $\left.J_{C-F}=266.6 \mathrm{~Hz}\right), 157.0\left(\mathrm{~d}, J_{C-F}=246.1 \mathrm{~Hz}\right), 133.7\left(\mathrm{~d}, J_{C-F}=2.6 \mathrm{~Hz}\right), 132.92,132.85$, $132.6\left(\mathrm{~d}, J_{C-F}=29.4 \mathrm{~Hz}\right), 130.4,130.3,129.6,128.46-128.23(\mathrm{~m}, 6 \mathrm{C}), 126.8\left(\mathrm{~d}, J_{C-F}=2.1\right.$ $\mathrm{Hz}), 123.9\left(\mathrm{~d}, J_{C-F}=7.0 \mathrm{~Hz}\right), 106.2\left(\mathrm{~d}, J_{C-F}=8.6 \mathrm{~Hz}\right), 105.5\left(\mathrm{~d}, J_{C-F}=17.7 \mathrm{~Hz}\right), 64.8,64.5$, 
$32.7\left(\mathrm{~d}, J_{C-F}=26.8 \mathrm{~Hz}\right), 28.3,28.0,25.9\left(\mathrm{~d}, J_{C-F}=1.8 \mathrm{~Hz}\right), 23.8\left(\mathrm{~d}, J_{C-F}=4.9 \mathrm{~Hz}\right), 23.0$; ${ }^{19} \mathrm{~F}$ NMR $\left(470 \mathrm{MHz}, \mathrm{CDCl}_{3}\right) \delta(\mathrm{ppm})=-101.5(\mathrm{dt}, J=37.2,18.9 \mathrm{~Hz}, 0.62 \mathrm{~F}, 2 \mathrm{~m}),-120.5$ (d, $J=37.5 \mathrm{~Hz}, 0.38 \mathrm{~F}, 2 \mathrm{~m}$ '); HRMS (APPI-TOF) $\mathrm{m} / \mathrm{z}$ calcd for $\mathrm{C}_{19} \mathrm{H}_{23} \mathrm{FNO}_{2}\left[\mathrm{M}+\mathrm{NH}_{4}\right]^{+}$ 316.1707; found 316.1702. The major regioisomer was identified from the expected coupling pattern in ${ }^{19} \mathrm{~F}$ NMR (doublet of triplet). The $\mathrm{Z}$ stereoisomers are established by the coupling constant between vinylic fluorine and proton $(37.2$ and $37.5 \mathrm{~Hz}=$ trans coupling). ${ }^{22}$

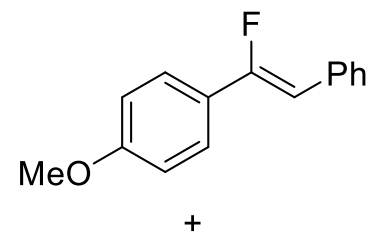

${ }_{\mathrm{MeO}}^{\mathrm{Ph}}$
(Z)-1-(1-Fluoro-2-phenylvinyl)-4-methoxybenzene + (Z)-1-(2Fluoro-2-phenylvinyl)-4-methoxybenzene $(74: 26)(2 n+2 n ')$

Prepared according to the general procedure on a $0.161 \mathrm{mmol}$ scale, the desired product (mixture or regioisomers 74:26, $25.7 \mathrm{mg}$, $0.113 \mathrm{mmol}, 70 \%$ ) was isolated as an off-white solid after purification by flash column chromatography $(2 \%$ EtOAc/hexanes). Mp: 84.2-86.0 ${ }^{\circ} \mathrm{C}$. FT-IR $v\left(\mathrm{~cm}^{-1}\right)=3001,2837,1651,1607,1512,1447$, 1298, 1250, 1178, 1036; ${ }^{1} \mathrm{H}$ NMR $\left(500 \mathrm{MHz}, \mathrm{CDCl}_{3}\right) \delta(\mathrm{ppm})=7.64-7.54(\mathrm{~m}, 4 \mathrm{H}, \mathbf{2 n}+$ 2n'), 7.42-7.20 (m, 3H, 2n + 2n'), 6.95-6.87 (m, 2H, 2n + 2n'), 6.26 (d, J = 39.9 Hz, 0.27H, 2n'), 6.17 (d, $J=39.9 \mathrm{~Hz}, 0.75 \mathrm{H}, \mathbf{2 n}), 3.83$ (s, 2.16H, 2n), 3.82 (s, 0.84H, 2n'); ${ }^{13} \mathrm{C}$ NMR $\left(101 \mathrm{MHz}^{\mathrm{CDCl}} \mathrm{CDC}_{3}\right) \delta(\mathrm{ppm})=160.3(\mathbf{2 n}), 158.8\left(\mathrm{~d}, J_{C-F}=3.1 \mathrm{~Hz}, \mathbf{2 n}\right.$ '), $157.3\left(\mathrm{~d}, J_{C-F}=\right.$ $257.8 \mathrm{~Hz}, 2 \mathbf{2 n}), 156.0$ (d, $\left.J_{C-F}=255.7 \mathrm{~Hz}, 2 \mathbf{n}^{\prime}\right), 134.0$ (d, $\left.J_{C-F}=3.0 \mathrm{~Hz}, 2 \mathbf{n}\right), 133.1$ (d, $J_{C-F}$ $=27.8 \mathrm{~Hz}, \mathbf{2 n}$ '), 131.5 (2n'), 130.3 (d, $J_{C-F}=8.1 \mathrm{~Hz}, \mathbf{2 n}$ '), 128.7 (d, $\left.J_{C-F}=7.9 \mathrm{~Hz}, 2 \mathbf{n}\right)$, $128.60\left(\mathrm{~d}, J_{C-F}=3.1 \mathrm{~Hz}, 2 \mathrm{n}\right.$ ), $128.56(2 \mathrm{n}), 127.0\left(\mathrm{~d}, J_{C-F}=2.5 \mathrm{~Hz}, 2 \mathrm{n}\right), 126.4$ (d, $J_{C-F}=$ $3.0 \mathrm{~Hz}, 2$ n'), 125.9 (d, $\left.J_{C-F}=7.6 \mathrm{~Hz}, \mathbf{2 n}\right), 125.5$ (d, $\left.J_{C-F}=28.4 \mathrm{~Hz}, 2 \mathbf{2 n}\right), 124.0$ (d, $J_{C-F}=$ $7.5 \mathrm{~Hz}, 2$ n'), 114.1 (2n), 114.0 (2n'), 105.4 (d, $J_{C-F}=10.8 \mathrm{~Hz}, 2$ n'), 104.1 (d, $J_{C-F}=10.7$ $\mathrm{Hz}, 2 \mathrm{2n}), 55.4$ (2n), 55.3 (2n'); ${ }^{19} \mathrm{~F}$ NMR (470 MHz, $\left.\mathrm{CDCl}_{3}\right) \delta(\mathrm{ppm})=-113.2(\mathrm{~d}, J=39.7$ $\mathrm{Hz}, 0.74 \mathrm{~F}, \mathbf{2 n}$ ), -117.1 (d, $J=39.8 \mathrm{~Hz}, 0.26 \mathrm{~F}, \mathbf{2 n}$ '); HRMS (APPI-TOF) m/z calcd for $\mathrm{C}_{15} \mathrm{H}_{13} \mathrm{FO}\left[\mathrm{M}^{*}\right]^{+} 230.1009$; found 230.0996. Identification of regioisomers was made from known characterization of $\mathbf{2 n}$ '. ${ }^{23}$ The $\mathrm{Z}$ stereoisomers are confirmed by coupling constant between vinylic fluorine and proton $(39.7$ and $39.8 \mathrm{~Hz}=$ trans coupling $){ }^{22}$

\footnotetext{
${ }^{23}$ Xu, J.; Burton, D.J., J. Org. Chem. 2006, 71, 3743-3747.
} 
<smiles>O=[N+]([O-])c1ccc(/C=C(\F)c2ccccc2)cc1</smiles>

\section{(Z)-1-(2-Fluoro-2-phenylvinyl)-4-nitrobenzene (2o)}

Prepared according to the general procedure on a $0.178 \mathrm{mmol}$ scale, the desired product $(31.2 \mathrm{mg}, 0.128 \mathrm{mmol}, 72 \%)$ was isolated as an orange solid after purification by flash column chromatography (5\% Et $2 \mathrm{O} /$ hexanes). ${ }^{19} \mathrm{~F} \mathrm{NMR}$ analysis of the crude mixture showed a 93:7 ratio of regioisomers, but only the major isomer was isolated. ${ }^{1} \mathrm{H}$ NMR $\left(500 \mathrm{MHz}, \mathrm{CDCl}_{3}\right) \delta(\mathrm{ppm})=8.26-8.19(\mathrm{~m}, 2 \mathrm{H}), 7.80-7.74(\mathrm{~m}, 2 \mathrm{H}), 7.72-7.65$ $(\mathrm{m}, 2 \mathrm{H}), 7.49-7.40(\mathrm{~m}, 3 \mathrm{H}), 6.39(\mathrm{~d}, J=38.2 \mathrm{~Hz}, 1 \mathrm{H}) ;{ }^{19} \mathrm{~F}$ NMR $\left(470 \mathrm{MHz}, \mathrm{CDCl}_{3}\right) \delta$ $(\mathrm{ppm})=-108.2(\mathrm{~d}, J=38.1 \mathrm{~Hz}, 1 \mathrm{~F})$. Data are in accordance with the one described in the literature. $^{24}$

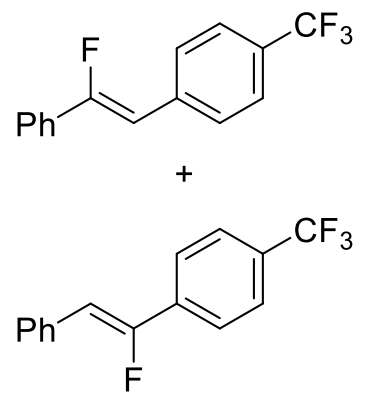

(Z)-1-(2-fluoro-2-phenylvinyl)-4-(trifluoromethyl) + (Z)-1-(1fluoro-2-phenylvinyl)-4-(trifluoromethyl)benzene $(81: 19)(2 p+$ 2p')

Prepared according to the general procedure on a $0.194 \mathrm{mmol}$ scale, the desired product (mixture of regioisomers 81:19, $37.5 \mathrm{mg}, 0.141$ mmol, 73\%) was isolated as a white solid after purification by flash column chromatography (hexanes). Mp: 105.3-107.2 ${ }^{\circ} \mathrm{C}$. FT-IR $v\left(\mathrm{~cm}^{-1}\right)=1655,1614$, 1414, 1323, 1283, 1171, 1157, 1111, 1070, 1011; ${ }^{1} \mathrm{H}$ NMR $\left(500 \mathrm{MHz}, \mathrm{CDCl}_{3}\right) \delta(\mathrm{ppm})=$ 7.79-7.73 (m, 2H, 2p + 2p'), 7.70-7.62 (m, 4H, 2p + 2p'), 7.49-7.40 (m, 2.72H, 2p + 2p'), 7.35-7.30 (m, 0.18, 2p'), 6.44 (d, $J=39.3 \mathrm{~Hz}, 0.19 \mathrm{H}, 2$ p'), 6.44 (d, $J=39.3 \mathrm{~Hz}, 0.81 \mathrm{H}$, 2p); ${ }^{13} \mathrm{C} \mathrm{NMR}\left(126 \mathrm{MHz}, \mathrm{CDCl}_{3}\right) \delta(\mathrm{ppm})=158.7\left(\mathrm{~d}, J_{C-F}=261.7 \mathrm{~Hz}\right), 155.7\left(\mathrm{~d}, J_{C-F}=\right.$ $258.2 \mathrm{~Hz}), 137.3-137.1(\mathrm{~m}), 136.2\left(\mathrm{~d}, J_{C-F}=29.1 \mathrm{~Hz}\right), 133.0\left(\mathrm{~d}, J_{C-F}=3.2 \mathrm{~Hz}\right), 132.2(\mathrm{~d}$, $\left.J_{C-F}=27.6 \mathrm{~Hz}\right), 131.8\left(\mathrm{~d}, J_{C-F}=7.2 \mathrm{~Hz}\right), 130.7\left(\mathrm{~d}, J_{C-F}=32.7 \mathrm{~Hz}\right), 129.7,129.2\left(\mathrm{~d}, J_{C-F}=\right.$ $8.0 \mathrm{~Hz}), 129.0\left(\mathrm{~d}, J_{C-F}=8.3 \mathrm{~Hz}\right), 128.73,128.71,128.0\left(\mathrm{~d}, J_{C-F}=2.6 \mathrm{~Hz}\right), 125.8-125.5$ $(\mathrm{m}), 125.5\left(\mathrm{q}, J_{C-F}=3.8 \mathrm{~Hz}\right), 124.6\left(\mathrm{~d}, J_{C-F}=7.6 \mathrm{~Hz}\right), 124.4\left(\mathrm{~d}, J_{C-F}=7.6 \mathrm{~Hz}\right), 124.2(\mathrm{q}$, $\left.J_{C-F}=271.8 \mathrm{~Hz}\right), 123.9\left(\mathrm{q}, J_{C-F}=272.1 \mathrm{~Hz}\right), 108.0\left(\mathrm{~d}, J_{C-F}=10.1 \mathrm{~Hz}\right), 104.6\left(\mathrm{~d}, J_{C-F}=\right.$ $10.1 \mathrm{~Hz}) ;{ }^{19} \mathrm{~F} \mathrm{NMR}\left(470 \mathrm{MHz}, \mathrm{CDCl}_{3}\right) \delta(\mathrm{ppm})=-62.6$ (s, 2.40F, 2p), -62.7 (s, 0.56F, 2p'), -111.1 (d, $J=38.2 \mathrm{~Hz}, 0.81 \mathrm{~F}, 2 \mathbf{2}),-115.2$ (d, $J=39.3 \mathrm{~Hz}, 0.19 \mathrm{~F}, 2$ p'); HRMS (APPI-TOF) $\mathrm{m} / \mathrm{z}$ calcd for $\mathrm{C}_{15} \mathrm{H}_{10} \mathrm{~F}_{4}\left[\mathrm{M}^{*}\right]^{+} 266.0713$; found 266.0701. The major isomer was proposed based on the results obtained with similar compounds (2o,r). The $\mathrm{Z}$ stereoisomers are

\footnotetext{
${ }^{24}$ Zhang, W.; Huang, W.; Hu, J., Angew. Chem. Int. Ed. 2009, 48, 9858-9861.
} 
confirmed by coupling constants between vinylic fluorine and proton $(38.2$ and $39.3 \mathrm{~Hz}=$ trans coupling). ${ }^{22}$

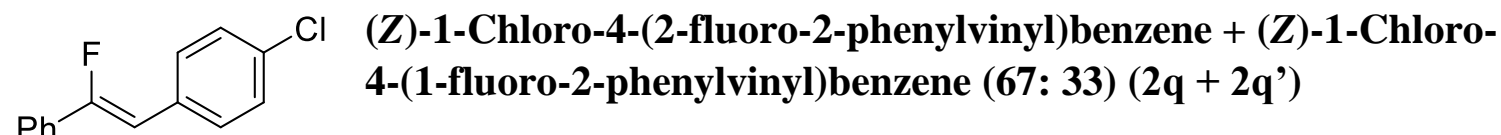

Prepared according to the general procedure on a $0.199 \mathrm{mmol}$ scale,<smiles>FC(=Cc1ccccc1)c1ccc(Cl)cc1</smiles>
the desired product (contaminated with 5\% residual starting material, mixture of regioisomers $67: 33,38.4 \mathrm{mg}, 0.165 \mathrm{mmol}, 83 \%$ ) was isolated as a white solid after purification by flash column chromatography (hexanes). FT-IR $v\left(\mathrm{~cm}^{-1}\right)=2982,2937,2924,1653,1497,1281,1092$, 1034, 1013, 905; z ${ }^{1} \mathrm{H}$ NMR (500 MHz, $\left.\mathrm{CDCl}_{3}\right) \delta(\mathrm{ppm})=7.66-7.60$ (m, 2H, 2q + 2q'), 7.59-7.53 (m, 2H, 2q + 2q') 7.44-7.24 (m, 5H, 2q + 2q'), 6.28 (d, J = 39.4 Hz, 0.34H, 2q'), $6.26(\mathrm{~d}, J=39.1 \mathrm{~Hz}, 0.66 \mathrm{H}, \mathbf{2 q}) ;{ }^{13} \mathrm{C} \mathrm{NMR}\left(126 \mathrm{MHz}, \mathrm{CDCl}_{3}\right) \delta(\mathrm{ppm})=157.6(\mathrm{~d}$, $\left.J_{C-F}=259.0 \mathrm{~Hz}\right), 156.2\left(\mathrm{~d}, J_{C-F}=258.0 \mathrm{~Hz}\right), 134.9,133.3\left(\mathrm{~d}, J_{C-F}=3.2 \mathrm{~Hz}\right), 132.9\left(\mathrm{~d}, J_{C-}\right.$ $F=3.7 \mathrm{~Hz}), 132.5\left(\mathrm{~d}, J_{C-F}=27.7 \mathrm{~Hz}\right), 132.1\left(\mathrm{~d}, J_{C-F}=2.9 \mathrm{~Hz}\right), 131.6,131.3\left(\mathrm{~d}, J_{C-F}=28.7\right.$ $\mathrm{Hz}), 130.1\left(\mathrm{~d}, J_{C-F}=8.4 \mathrm{~Hz}\right), 129.2,129.0\left(\mathrm{~d}, J_{C-F}=7.8 \mathrm{~Hz}\right), 128.8\left(\mathrm{~d}, J_{C-F}=2.0 \mathrm{~Hz}\right)$, $128.7\left(\mathrm{~d}, J_{C-F}=14.2 \mathrm{~Hz}\right), 128.7,127.6\left(\mathrm{~d}, J_{C-F}=2.4 \mathrm{~Hz}\right), 125.5\left(\mathrm{~d}, J_{C-F}=7.5 \mathrm{~Hz}\right), 124.3$ $\left(\mathrm{d}, J_{C-F}=7.6 \mathrm{~Hz}\right), 106.3\left(\mathrm{~d}, J_{C-F}=10.4 \mathrm{~Hz}\right), 104.7\left(\mathrm{~d}, J_{C-F}=10.4 \mathrm{~Hz}\right) ;{ }^{19} \mathrm{~F}$ NMR $(470$ $\left.\mathrm{MHz} \mathrm{CDCl}_{3}\right) \delta(\mathrm{ppm})=-113.4(\mathrm{~d}, J=38.3 \mathrm{~Hz}, 0.67 \mathrm{~F}, 2 \mathrm{q}),-114.5(\mathrm{~d}, J=39.3 \mathrm{~Hz}, 0.33 \mathrm{~F}$, 2q'); HRMS (APPI-TOF) m/z calcd for $\mathrm{C}_{14} \mathrm{H}_{10} \mathrm{ClF}$ [M*] ${ }^{+} 232.0450$; found 232.0443. The major isomer was proposed based on the results obtained with similar compounds $(\mathbf{2 o}, \mathbf{r})$. Spectral data also agreed with the ones from literature for the major isomer. ${ }^{25}$ The $\mathrm{Z}$ stereoisomers are confirmed by coupling constants between vinylic fluorine and proton $(38.3$ and $39.3 \mathrm{~Hz}=$ trans coupling $){ }^{22}$

\footnotetext{
${ }^{25}$ Tsai, H.-J.; Lin, K.-W.; Ting, T.-H.; Burton D.J., Helv. Chim. Acta 1999, 82, 2231-2239.
} 
<smiles>CC(=O)c1ccc(/C=C(\F)c2ccc(C(C)=O)cc2)cc1</smiles>

(Z)-1-Chloro-4-(2-fluoro-2-phenylvinyl)benzene + (Z)-1Chloro-4-(1-fluoro-2-phenylvinyl)benzene (84:16) $\left(2 r+2 r^{\prime}\right)$

Prepared according to the general procedure on a $0.193 \mathrm{mmol} \mathrm{scale}$, the desired product (mixture of regioisomers 84:16, $37.5 \mathrm{mg}, 0.156$ mmol, $81 \%$ ) was isolated as a pale yellow solid after purification by flash column chromatography (5-15\% EtOAc/hexanes). Mp: 140.8$147.8{ }^{\circ} \mathrm{C}$. FT-IR $v\left(\mathrm{~cm}^{-1}\right)=3003,1676,1603,1448,1410,1360$, 1267, 1186, 1034, 1011; ${ }^{1} \mathrm{H}$ NMR $\left(500 \mathrm{MHz} \mathrm{CDCl}_{3}\right) \delta(\mathrm{ppm})=8.02-7.94(\mathrm{~m}, 2 \mathrm{H}, \mathbf{2 r}+$ 2r'), 7.76-7.69 (m, 2H, 2r + 2r'), 7.70-7.63 (m, 2H, 2r + 2r'), 7.47-7.36 (m, 2.71H, 2r + 2r'), 7.34-7.27 (m, 0.20H, 2r'), 6.45 (d, $\left.J=39.2 \mathrm{~Hz}, 0.16 \mathrm{H}, 2 \mathbf{r}^{\prime}\right), 6.36$ (d, $J=38.9 \mathrm{~Hz}$, 0.84H, 2r), 2.62 (s, 0.67H, 2r'), 2.61 (s, 2.27H, 2r); $\left.{ }^{13} \mathrm{C} \mathrm{NMR} \mathrm{(126} \mathrm{MHz,} \mathrm{CDCl}_{3}\right) \delta$ (ppm) = 197.5 (2r), 197.3 (2r'), 158.8 (d, $J_{C-F}=262.5 \mathrm{~Hz}, \mathbf{2 r}$ ), 156.0 (d, $J_{C-F}=258.2 \mathrm{~Hz}, 2$ r') $)$, $138.5\left(\mathrm{~d}, J_{C-F}=3.3 \mathrm{~Hz}, 2 \mathbf{2 r}\right), 137.2$ (2r'), 136.9 (2r), 135.4 (d, $\left.J_{C-F}=2.4 \mathrm{~Hz}, 2 \mathbf{2 r}\right), 133.1$ (d, $J_{C-F}=3.2 \mathrm{~Hz}, \mathbf{2 r}$ '), 132.3 (d, $\left.J_{C-F}=27.6 \mathrm{~Hz}, \mathbf{2 r}\right), 129.7$ (2r), 129.3 (d, $\left.J_{C-F}=8.1 \mathrm{~Hz}, 2 \mathbf{r}^{\prime}\right)$, $128.9\left(\mathrm{~d}, J_{C-F}=8.3 \mathrm{~Hz}, \mathbf{2 r}\right), 128.71$ (d, $J_{C-F}=2.2 \mathrm{~Hz}, \mathbf{2 r}$ '), 128.70 (2r'), 128.69 (2r'), $128.67(\mathbf{2 r}), 128.0$ (d, $\left.J_{C-F}=2.6 \mathrm{~Hz}, 2 \mathbf{r}^{\prime}\right), 124.6$ (d, $\left.J_{C-F}=7.6 \mathrm{~Hz}, 2 \mathbf{2}\right), 124.2\left(\mathrm{~d}, J_{C-F}=7.5\right.$ Hz, 2r'), 108.3 (d, $J_{C-F}=10.1 \mathrm{~Hz}, 2$ r'), 105.0 (d, $\left.J_{C-F}=10.0 \mathrm{~Hz}, 2 \mathbf{2 r}\right), 26.7$ (2r'), 26.6 (2r); ${ }^{19} \mathrm{~F} \mathrm{NMR}\left(470 \mathrm{MHz}, \mathrm{CDCl}_{3}\right) \delta(\mathrm{ppm})=-110.2(\mathrm{~d}, J=38.3 \mathrm{~Hz}, 0.84 \mathrm{~F}, 2 \mathrm{r}),-115.2(\mathrm{~d}, J=$ $38.4 \mathrm{~Hz}, 0.16 \mathrm{~F}, 2 \mathbf{r}^{\prime}$ ); HRMS (APPI-TOF) m/z calcd for $\mathrm{C}_{16} \mathrm{H}_{14} \mathrm{FO}[\mathrm{M}+\mathrm{H}]^{+} 241.1023$; found 241.1006. Identification of regioisomers was made from known characterization of 2r'. ${ }^{26}$ The $\mathrm{Z}$ stereoisomers are established by the coupling constant between vinylic fluorine and proton $\left(38.3\right.$ and $38.4 \mathrm{~Hz}=$ trans coupling). ${ }^{22}$
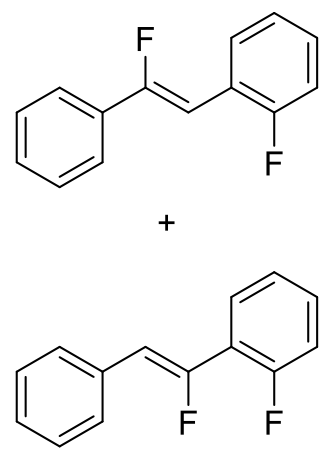

(Z)-1-fluoro-2-(2-fluoro-2-phenylvinyl)benzene $+(Z)$-1-fluoro-2(1-fluoro-2-phenylvinyl)benzene (77:23) $\left(2 \mathrm{~s}+2 \mathrm{~s}^{\prime}\right)$

Prepared according to the general procedure on a $0.195 \mathrm{mmol} \mathrm{scale}$, the desired product (mixture of regioisomers 77:23, $21.2 \mathrm{mg}, 0.098$ mmol, 50\%) was isolated as a white solid after purification by flash column chromatography (hexanes). Mp: 53.1-53.8 ${ }^{\circ} \mathrm{C}$; FT-IR $v\left(\mathrm{~cm}^{-}\right.$ $\left.{ }^{1}\right)=3067,2924,1657,1578,1497,1483,1454,1229,1040,1014 ;{ }^{1} \mathrm{H}$

${ }^{26}$ Chen, C.; Wilcoxen, K.; Zhu, Y.; Kim, K.; McCarthy J.R., J. Org. Chem. 1999, 64, 3476-3482. 
$\operatorname{NMR}\left(500 \mathrm{MHz}, \mathrm{CDCl}_{3}\right) \delta(\mathrm{ppm})=8.03-7.94(\mathrm{~m}, 0.77 \mathrm{H}, \mathbf{2 s}), 7.71-7.62(\mathrm{~m}, 2.29 \mathrm{H}, \mathbf{2 s}+$ 2s'), 7.46-7.27 (m, 3.30H, 2s + 2s's), 7.25-7.04 (m, 2.75H, 2s + 2s'), 6.60 (d, J = 39.5 Hz, $0.77 \mathrm{H}, 2 \mathrm{~s}), 6.53\left(\mathrm{~d}, J=41.6 \mathrm{~Hz}, 0.23 \mathrm{H}, 2 \mathbf{s}^{\prime}\right) ;{ }^{13} \mathrm{C} \mathrm{NMR}\left(101 \mathrm{MHz}, \mathrm{CDCl}_{3}\right) \delta(\mathrm{ppm})=159.8$ $\left(\mathrm{dd}, J_{C-F}=249.3,1.3 \mathrm{~Hz}\right), 158.3\left(\mathrm{dd}, J_{C-F}=260.2,2.4 \mathrm{~Hz}\right), 133.6\left(\mathrm{~d}, J_{C-F}=2.7 \mathrm{~Hz}\right), 132.7$, $130.24,130.18,130.15,130.0\left(\mathrm{~d}, J_{C-F}=2.5 \mathrm{~Hz}\right), 129.32,129.25,128.74\left(\mathrm{~d}, J_{C-F}=2.2 \mathrm{~Hz}\right)$, $128.66\left(\mathrm{~d}, J_{C-F}=2.0 \mathrm{~Hz}\right), 128.63\left(\mathrm{~d}, J_{C-F}=2.1 \mathrm{~Hz}\right), 128.56,127.6\left(\mathrm{~d}, J_{C-F}=2.5 \mathrm{~Hz}\right), 127.3$ $\left(\mathrm{dd}, J_{C-F}=9.3,2.1 \mathrm{~Hz}\right), 124.5\left(\mathrm{~d}, J_{C-F}=7.5 \mathrm{~Hz}\right), 124.3\left(\mathrm{~d}, J_{C-F}=1.6 \mathrm{~Hz}\right), 124.2\left(\mathrm{~d}, J_{C-F}=\right.$ $3.5 \mathrm{~Hz}), 121.6\left(\mathrm{dd}, J_{C-F}=11.8,3.2 \mathrm{~Hz}\right), 116.3\left(\mathrm{dd}, J_{C-F}=22.9,2.6 \mathrm{~Hz}\right), 115.2\left(\mathrm{dd}, J_{C-F}=\right.$ 22.2, $1.0 \mathrm{~Hz}), 111.6\left(\mathrm{dd}, J_{C-F}=13.9,8.9 \mathrm{~Hz}\right), 97.0\left(\mathrm{dd}, J_{C-F}=9.5,7.5 \mathrm{~Hz}\right) ;{ }^{19} \mathrm{~F} \mathrm{NMR}(470$ $\left.\mathrm{MHz} \mathrm{CDCl}_{3}\right) \delta(\mathrm{ppm})=-110.4\left(\mathrm{dd}, J=41.5,8.6 \mathrm{~Hz}, 0.23 \mathrm{~F}, 2 \mathrm{~s}^{\prime}\right),-111.7(\mathrm{dtd}, J=12.5$, 8.2, 4.8 Hz, 0.23F, 2s'), -112.8 (dd, $J=39.4,3.9 \mathrm{~Hz}, 0.77 \mathrm{~F}, 2 \mathrm{~s}$ ), -116.7 (ddt, $J=11.7,8.5$, $4.4 \mathrm{~Hz}, 0.77 \mathrm{~F}, 2 \mathrm{~s}$ ); HRMS (ESI-TOF) $\mathrm{m} / \mathrm{z}$ calcd for $\mathrm{C}_{14} \mathrm{H}_{11} \mathrm{~F}_{2}[\mathrm{M}+\mathrm{H}]^{+} 217.0823$; found 217.0834. The identity of the regioisomers was proposed based on the coupling constant between the two fluorine atoms $\left(8.6 \mathrm{~Hz}\right.$ means ${ }^{4} J$ and $3.9 \mathrm{~Hz}$ means $\left.{ }^{5} J\right) .{ }^{22}$ The $\mathrm{Z}$ geometry was established from the coupling constant between the vinylic fluorine and the vinylic proton $(41.5$ and $39.4 \mathrm{~Hz}=$ trans coupling $){ }^{22}$

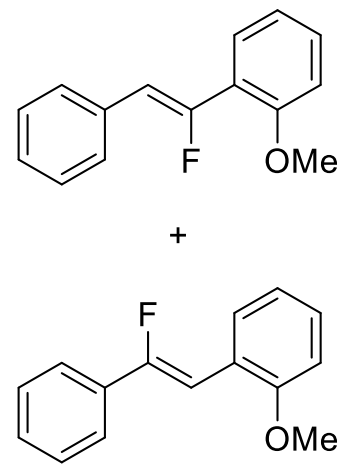

\section{(Z)-1-(1-Fluoro-2-phenylvinyl)-2-methoxybenzene + (Z)-1-(2- Fluoro-2-phenylvinyl)-2-methoxybenzene $(63: 37)\left(2 t+2 t^{\prime}\right)$}

Prepared according to the general procedure on a $0.206 \mathrm{mmol}$ scale, the desired product (mixture of regioisomers 63:37, $17.4 \mathrm{mg}, 0.076$ mmol, 40\%) was isolated as a colorless oil after purification by flash column chromatography $(25 \%$ toluene/hexanes $)$. FT-IR $v\left(\mathrm{~cm}^{-1}\right)=$ 2937, 2837, 1651, 1597, 1576, 1493, 1464, 1435, 1257, 1244; ${ }^{1} \mathrm{H}$

NMR $\left(500 \mathrm{MHz} \mathrm{CDCl}_{3}\right) \delta(\mathrm{ppm})=7.95(\mathrm{dd}, J=7.8,1.7 \mathrm{~Hz}, 0.37 \mathrm{H}, \mathbf{2 t}$ ') $7.71-7.59(\mathrm{~m}$, 2.72H, 2t), 7.43-7.30 (m, 3H, 2t + 2t' ') 7.27-7.20 (m, 1H, 2t + 2t' '), 7.04-6.87 (m, 2H, 2t + 2t') 6.79 (d, $J=40.8 \mathrm{~Hz}, 0.38 \mathrm{H}, \mathbf{2 t}$ '), 6.68 (d, $J=42.4 \mathrm{~Hz}, 0.62 \mathrm{H}, \mathbf{2 t}), 3.93$ (s, $1.97 \mathrm{H}$, 2t), $3.87\left(\mathrm{~s}, 1.13 \mathrm{H}, \mathbf{2 t} \mathbf{t}^{\prime}\right) ;{ }^{13} \mathrm{C}$ NMR $\left(126 \mathrm{MHz}, \mathrm{CDCl}_{3}\right) \delta(\mathrm{ppm})=157.1\left(\mathrm{~d}, J_{C-F}=257.8\right.$ $\mathrm{Hz}), 156.6\left(\mathrm{~d}, J_{C-F}=5.4 \mathrm{~Hz}\right), 156.44,156.43,154.2\left(\mathrm{~d}, J_{C-F}=256.3 \mathrm{~Hz}\right), 134.4\left(\mathrm{~d}, J_{C-F}=\right.$ $2.3 \mathrm{~Hz}), 133.3\left(\mathrm{~d}, J_{C-F}=28.2 \mathrm{~Hz}\right), 130.0,129.9\left(\mathrm{~d}, J_{C-F}=13.9 \mathrm{~Hz}\right), 129.2\left(\mathrm{~d}, J_{C-F}=8.0\right.$ $\mathrm{Hz}), 128.7,128.49,128.48,128.46,128.4,127.6\left(\mathrm{~d}, J_{C-F}=10.3 \mathrm{~Hz}\right), 127.0\left(\mathrm{~d}, J_{C-F}=2.4\right.$ 
$\mathrm{Hz}), 124.3\left(\mathrm{~d}, J_{C-F}=7.5 \mathrm{~Hz}\right), 122.5\left(\mathrm{~d}, J_{C-F}=3.4 \mathrm{~Hz}\right), 121.7\left(\mathrm{~d}, J_{C-F}=27.3 \mathrm{~Hz}\right), 111.2(\mathrm{~d}$, $\left.J_{C-F}=2.3 \mathrm{~Hz}\right), 110.8\left(\mathrm{~d}, J_{C-F}=9.2 \mathrm{~Hz}\right), 110.4,99.3\left(\mathrm{~d}, J_{C-F}=8.7 \mathrm{~Hz}\right), 55.60,55.56 ;{ }^{19} \mathrm{~F}$ $\operatorname{NMR}\left(470 \mathrm{MHz}, \mathrm{CDCl}_{3}\right) \delta(\mathrm{ppm})=-106.5(\mathrm{~d}, J=42.4 \mathrm{~Hz}, 0.63 \mathrm{~F}, 2 \mathrm{t}),-116.0(\mathrm{~d}, J=40.8$ $\mathrm{Hz}, 0.37 \mathrm{~F}, 2 \mathbf{t}$; HRMS (ESI-TOF) $\mathrm{m} / \mathrm{z}$ calcd for $\mathrm{C}_{15} \mathrm{H}_{14} \mathrm{FO}[\mathrm{M}+\mathrm{H}]^{+} 229.1023$; found 229.1010. The identity of the regioisomers was proposed based on HOESY and COSY experiments. The $\mathrm{Z}$ geometry was established from the coupling constant between the vinylic fluorine and the vinylic proton $\left(42.4\right.$ and $40.8 \mathrm{~Hz}=$ trans coupling). ${ }^{22}$<smiles>F/C(=C\Cl)CCc1ccccc1</smiles>
(Z)-(4-Chloro-3-fluorobut-3-en-1-yl)benzene (2u)

Prepared according to the general procedure on a $0.204 \mathrm{mmol}$ scale, the desired product (23.1 $\mathrm{mg}, 0.125 \mathrm{mmol}, 61 \%$ ) was isolated as a colourless oil after purification by flash column chromatography (hexanes). ${ }^{1} \mathrm{H}$ NMR $\left(500 \mathrm{MHz}, \mathrm{CDCl}_{3}\right) \delta$ $(\mathrm{ppm})=7.34-7.27(\mathrm{~m}, 2 \mathrm{H}), 7.24-7.20(\mathrm{~m}, 1 \mathrm{H}), 7.19-7.15(\mathrm{~m}, 2 \mathrm{H}), 5.28(\mathrm{dt}, J=24.6,0.9$ $\mathrm{Hz}, 1 \mathrm{H}), 2.87-2.82(\mathrm{~m}, 2 \mathrm{H}), 2.58-2.48(\mathrm{~m}, 2 \mathrm{H}) ;{ }^{13} \mathrm{C} \mathrm{NMR}\left(126 \mathrm{MHz}, \mathrm{CDCl}_{3}\right) \delta(\mathrm{ppm})=$ $160.0\left(\mathrm{~d}, J_{C-F}=261.4 \mathrm{~Hz}\right), 139.9,128.6,126.4,96.7\left(\mathrm{~d}, J_{C-F}=18.5 \mathrm{~Hz}\right), 33.1\left(\mathrm{~d}, J_{C-F}=\right.$ $24.4 \mathrm{~Hz}), 32.1 ;{ }^{19} \mathrm{~F} \mathrm{NMR}\left(470 \mathrm{MHz}, \mathrm{CDCl}_{3}\right) \delta(\mathrm{ppm})=-104.9(\mathrm{dt}, J=24.2,18.2 \mathrm{~Hz}, 1 \mathrm{~F})$; HRMS (APPI-TOF) $\mathrm{m} / \mathrm{z}$ calcd for $\mathrm{C}_{10} \mathrm{H}_{11} \mathrm{ClFNa}[\mathrm{M}+\mathrm{Na}]^{+}$204.0347; found 207.0347.

$\mathrm{Br}$-(4-Bromo-3-fluorobut-3-en-1-yl)benzene (2v)
Prepared according to the general procedure on a $0.202 \mathrm{mmol}$ scale, the desired product $(22.4 \mathrm{mg}, 0.098 \mathrm{mmol}, 48 \%)$ was isolated as a colourless oil after purification by flash column chromatography (hexanes). FT-IR $v\left(\mathrm{~cm}^{-1}\right)=3103,3028$, $2932,1672,1605,1497,1454,1273,1138,883 ;{ }^{1} \mathrm{H}$ NMR $\left(500 \mathrm{MHz}, \mathrm{CDCl}_{3}\right) \delta(\mathrm{ppm})=$ 7.33-7.28 (m, 2H), 7.25-7.20 (m, 1H), 7.19-7.15 (m, 2H), 5.28 (d, $J=28.1 \mathrm{~Hz}, 1 \mathrm{H}), 2.85$ (t, $J=8.1 \mathrm{~Hz}, 2 \mathrm{H}), 2.56(\mathrm{dt}, J=17.1,7.7 \mathrm{~Hz}, 2 \mathrm{H}) ;{ }^{13} \mathrm{C} \mathrm{NMR}\left(126 \mathrm{MHz}, \mathrm{CDCl}_{3}\right) \delta(\mathrm{ppm})$ $=161.5\left(\mathrm{~d}, J_{C-F}=261.1 \mathrm{~Hz}\right), 139.8,128.6,128.3,126.5,83.2\left(\mathrm{~d}, J_{C-F}=21.6 \mathrm{~Hz}\right), 34.0(\mathrm{~d}$, $\left.J_{C-F}=25.5 \mathrm{~Hz}\right), 32.1 ;{ }^{19} \mathrm{~F} \mathrm{NMR}\left(470 \mathrm{MHz}, \mathrm{CDCl}_{3}\right) \delta(\mathrm{ppm})=-95.7(\mathrm{dt}, J=28.0,17.3 \mathrm{~Hz}$, 1F); GC-MS (EI) m/z calcd for $\mathrm{C}_{10} \mathrm{H}_{10} \mathrm{~F}$ [M-Br] ${ }^{+} 149.08$; found 149.13. Under all other conditions tested for MS analysis (HRMS-ESI(+). HRMS-ESI(-), HRMS-APPI and GCMS-CI), we never could detect any significant ions. 
F O Ethyl (Z)-3-fluoro-3-phenylacrylate (2w)

OEt Prepared according to the general procedure on a $0.195 \mathrm{mmol}$ scale, the desired product $(12.7 \mathrm{mg}, 0.065 \mathrm{mmol}, 34 \%)$ was isolated as a colourless oil after purification by automated flash purification (0-15\% EtOAc/hexanes). ${ }^{1} \mathrm{H}$ NMR $(500 \mathrm{MHz}$, $\left.\mathrm{CDCl}_{3}\right) \delta(\mathrm{ppm})=7.68-7.64(\mathrm{~m}, 2 \mathrm{H}), 7.51-7.41(\mathrm{~m}, 3 \mathrm{H}), 5.91(\mathrm{~d}, J=33.3 \mathrm{~Hz}, 1 \mathrm{H}), 4.26$ $(\mathrm{q}, J=7.1 \mathrm{~Hz}, 3 \mathrm{H}), 1.34(\mathrm{t}, J=7.1 \mathrm{~Hz}, 3 \mathrm{H}) ;{ }^{19} \mathrm{~F} \mathrm{NMR}\left(470 \mathrm{MHz}, \mathrm{CDCl}_{3}\right) \delta(\mathrm{ppm})=-96.2$ $(\mathrm{d}, J=33.1 \mathrm{~Hz}, 1 \mathrm{~F})$. Data are in accordance with the one described in the literature. ${ }^{2}$

\section{O O Ethyl 3-oxo-3-phenylpropanoate}

OEt Side product of the hydrofluorination of $1 \mathbf{u}$ on $0.195 \mathrm{mmol}$ scale. The side product (18.9 mg, $0.098 \mathrm{mmol}, 50 \%)$ was isolated as a colourless oil after purification by automated flash purification (0-15\% EtOAc/hexanes). ${ }^{1} \mathrm{H}$ NMR (500 $\mathrm{MHz}, \mathrm{CDCl}_{3}$, mixture of tautomers $) \delta(\mathrm{ppm})=12.59(\mathrm{~s}, 0.18 \mathrm{H}), 7.98-7.93(\mathrm{~m}, 1.54 \mathrm{H}), 7.80-7.76(\mathrm{~m}$, 0.39), 7.62-7.58 (m, 0.78H), 7.51-7.39 (m, 2.16H), $5.67(\mathrm{~s}, 0.18 \mathrm{H}), 4.27(\mathrm{q}, J=7.1 \mathrm{~Hz}$, $0.42 \mathrm{H}), 4.22(\mathrm{q}, J=7.1 \mathrm{~Hz}, 1.58), 4.00(\mathrm{~s}, 1.63 \mathrm{H}), 1.34(\mathrm{t}, J=7.1 \mathrm{~Hz}, 0.58 \mathrm{H}), 1.26(\mathrm{t}, J=$ 7.1 Hz, 2.46H). Spectral data are identical to the one of an authentic commercial sample.<smiles>C=C(F)CC(C(=O)OCC)C(=O)OCC</smiles>

\section{Diethyl 2-(2-fluoroallyl)malonate (2z)}

Prepared according to the general procedure on a $0.148 \mathrm{mmol}$ scale, the desired product (10.8 $\mathrm{mg}, 0.049 \mathrm{mmol}, 26 \%$ ) was isolated as a colourless oil after purification by automated flash purification $(0-15 \%$ EtOAc/hexanes). FT-IR $v\left(\mathrm{~cm}^{-1}\right)=2984,1749,1732,1678,1369,1333,1259,1217,1149$, 1034; ${ }^{1} \mathrm{H}$ NMR $\left(500 \mathrm{MHz}, \mathrm{CDCl}_{3}\right) \delta(\mathrm{ppm})=4.60(\mathrm{dd}, J=17.1,3.1 \mathrm{~Hz}, 1 \mathrm{H}), 4.35(\mathrm{dd}, J$ $=49.4,3.1 \mathrm{~Hz}, 1 \mathrm{H}), 4.22(\mathrm{qd}, J=7.1,2.2 \mathrm{~Hz}, 4 \mathrm{H}), 3.62(\mathrm{t}, J=7.6 \mathrm{~Hz}, 1 \mathrm{H}), 2.82(\mathrm{dd}, J=$ 16.8, 7.6 Hz, 2H), 1.28 (t, $J=7.1 \mathrm{~Hz}, 6 \mathrm{H}) ;{ }^{13} \mathrm{C} \mathrm{NMR}\left(126 \mathrm{MHz}, \mathrm{CDCl}_{3}\right) \delta(\mathrm{ppm})=168.3$, $162.8\left(\mathrm{~d}, J_{C-F}=256.5 \mathrm{~Hz}\right), 92.2\left(\mathrm{~d}, J_{C-F}=19.4 \mathrm{~Hz}\right), 61.8,49.1,31.3\left(\mathrm{~d}, J_{C-F}=28.6 \mathrm{~Hz}\right)$, 14.0; ${ }^{19} \mathrm{~F}$ NMR $\left(470 \mathrm{MHz}, \mathrm{CDCl}_{3}\right) \delta(\mathrm{ppm})=-97.0(\mathrm{dtd}, J=49.8,16.9,16.6 \mathrm{~Hz}, 1 \mathrm{~F})$; HRMS (ESI-TOF) m/z calcd for $\mathrm{C}_{10} \mathrm{H}_{16} \mathrm{FO}_{4}[\mathrm{M}+\mathrm{H}]^{+} 220.1061$; found 220.1085. 


\section{F F F $\quad(Z)-(1,3,3-$ trifluorohex-1-en-1-yl)benzene (4a)}

$\mathrm{Ph}^{-}$

Prepared according to the general procedure on a $0.174 \mathrm{mmol}$ scale.

${ }^{19} \mathrm{~F}$ NMR analysis of the crude mixture showed a yield of $80 \%$. The desired product $(21.2$ $\mathrm{mg}, 0.099 \mathrm{mmol}, 57 \%$ ) was isolated as a colourless oil after purification by flash column chromatography (hexanes). FT-IR $v\left(\mathrm{~cm}^{-1}\right)=2964,2935,1701,1661,1641,1622,1448$, 1325, 1192, 1053; ${ }^{1} \mathrm{H}$ NMR (500 MHz, $\left.\mathrm{CDCl}_{3}\right) \delta(\mathrm{ppm})=7.58-7.54(\mathrm{~m}, 2 \mathrm{H}), 7.44-7.38$ $(\mathrm{m}, 3 \mathrm{H}), 5.62(\mathrm{dt}, J=37.2,11.0 \mathrm{~Hz}, 1 \mathrm{H}), 2.20-2.06(\mathrm{~m}, 2 \mathrm{H}), 1.63-1.52(\mathrm{~m}, 2 \mathrm{H}), 1.00(\mathrm{t}, J$ $=7.4 \mathrm{~Hz}, 3 \mathrm{H}) ;{ }^{13} \mathrm{C} \mathrm{NMR}\left(126 \mathrm{MHz}, \mathrm{CDCl}_{3}\right) \delta(\mathrm{ppm})=159.9\left(\mathrm{dt}, J_{C-F}=262.1,10.0 \mathrm{~Hz}\right)$, $130.8\left(\mathrm{~d}, J_{C-F}=27.6 \mathrm{~Hz}\right), 130.4,128.7\left(\mathrm{~d}, J_{C-F}=2.0 \mathrm{~Hz}\right), 121.6\left(\mathrm{t}, J_{C-F}=237.6 \mathrm{~Hz}\right), 102.3$ $\left(\mathrm{td}, J_{C-F}=31.9,11.9 \mathrm{~Hz}\right), 39.9\left(\mathrm{td}, J_{C-F}=26.0,2.9 \mathrm{~Hz}\right), 16.0\left(\mathrm{t}, J_{C-F}=4.3 \mathrm{~Hz}\right), 13.9 ;{ }^{19} \mathrm{~F}$ $\operatorname{NMR}\left(470 \mathrm{MHz}, \mathrm{CDCl}_{3}\right) \delta(\mathrm{ppm})=-87.8(\mathrm{tdd}, J=16.6,12.7,10.5 \mathrm{~Hz}, 2 \mathrm{~F}),-108.2(\mathrm{dt}, J$ $=36.8,13.5 \mathrm{~Hz}, 1 \mathrm{~F})$; HRMS (APPI-TOF) $\mathrm{m} / \mathrm{z}$ calcd for $\mathrm{C}_{12} \mathrm{H}_{13} \mathrm{~F}_{3} \mathrm{Na}[\mathrm{M}+\mathrm{Na}]^{+} 237.0862$; found 237.0836. Identification of the regioisomer was done using coupling pattern and constants of the vinylic proton, which indicates ${ }^{3} \mathrm{~J}_{\mathrm{HF}}$ coupling. $\mathrm{Z}$ stereoisomer is established by the coupling constant between vinylic fluorine and proton $(37.2 \mathrm{~Hz}=$ trans coupling). ${ }^{22}$

\section{$\mathrm{F} \quad(Z)-(3,5,5,5$-tetrafluoropent-3-en-1-yl)benzene (4b)}

$\mathrm{CF}_{3}$ Prepared according to the general procedure on a $0.229 \mathrm{mmol}$ scale. ${ }^{19} \mathrm{~F}$ NMR analysis of the crude mixture showed 2 stereoisomers (85:15, 76\% yield). Only the major isomer $(24.7 \mathrm{mg}, 0.113 \mathrm{mmol}, 49 \%)$ was isolated as a colourless oil after purification by flash column chromatography (hexanes). FT-IR $v\left(\mathrm{~cm}^{-1}\right)=3032,2935$, 1709, 1497, 1364, 1263, 1221, 1200, 1122, 1095; ${ }^{1} \mathrm{H}$ NMR $\left(500 \mathrm{MHz}, \mathrm{CDCl}_{3}\right) \delta(\mathrm{ppm})=$ 7.34-7.29 (m, 2H), 7.26-7.21 (m, 1H), 7.20-7.16 (m, 2H), 4.95 (dq, J = 33.4, 7.4 Hz, 1H), $2.87(\mathrm{t}, J=7.7 \mathrm{~Hz}, 2 \mathrm{H}), 2.60-2.51(\mathrm{~m}, 2 \mathrm{H}),{ }^{13} \mathrm{C} \mathrm{NMR}\left(126 \mathrm{MHz}, \mathrm{CDCl}_{3}\right) \delta(\mathrm{ppm})=166.9$ $\left(\mathrm{dq}, J_{C-F}=277.3,5.7 \mathrm{~Hz}\right), 139.2,128.7,128.3,126.7,122.0\left(\mathrm{q}, J_{C-F}=269.4 \mathrm{~Hz}\right), 97.9(\mathrm{qd}$, $\left.J_{C-F}=35.8,9.1 \mathrm{~Hz}\right), 34.1\left(\mathrm{~d}, J_{C-F}=24.0 \mathrm{~Hz}\right), 31.6 ;{ }^{19} \mathrm{~F} \mathrm{NMR}\left(470 \mathrm{MHz}, \mathrm{CDCl}_{3}\right) \delta(\mathrm{ppm})$ $=-57.8(\mathrm{dd}, J=16.5,7.4 \mathrm{~Hz}, 3 \mathrm{~F}),-89.3(\mathrm{dh}, J=33.6,16.9 \mathrm{~Hz}, 1 \mathrm{~F}) ; \mathrm{GC}-\mathrm{MS}(\mathrm{EI}) \mathrm{m} / \mathrm{z}$ calcd for $\mathrm{C}_{11} \mathrm{H}_{10} \mathrm{~F}_{4}[\mathrm{M}]^{+}$218.07; found 218.20. Under all other conditions tested for MS analysis (HRMS-ESI(+). HRMS-ESI(-), HRMS-APPI and GC-MS-CI), we never could detect any significant ions. The identification of the regioisomer is confirmed by the coupling constant 
between the vinylic proton, the vinylic fluorine and the trifluoromethyl group. The $\mathrm{Z}$ stereoisomer is established by the coupling constant between the vinylic proton and fluorine ( $33.4 \mathrm{~Hz}=$ trans coupling). ${ }^{22}$ On the crude ${ }^{19} \mathrm{~F} \mathrm{NMR}$, the minor isomer is identified as the $E$ stereoisomer from the same coupling constant $(17.3 \mathrm{~Hz}){ }^{22}$

\section{F (Z)-pentafluoro(2-fluoro-4-phenylbut-1-en-1-yl)- $\lambda^{6}$-sulfane (4c)}

$\mathrm{SF}_{5}$ Prepared according to the general procedure on a $0.233 \mathrm{mmol}$ scale. ${ }^{19} \mathrm{~F}$ NMR analysis of the crude mixture showed a yield of $26 \%$. The desired product (9.5 $\mathrm{mg}, 0.034 \mathrm{mmol}, 15 \%$ ) was isolated as a colourless oil after purification by automated flash purification (hexanes). FT-IR $v\left(\mathrm{~cm}^{-1}\right)=3032$, 2982, 2924, 1686, 1497, 1456, 1306, 1169 , 937, 835; ${ }^{1} \mathrm{H}$ NMR $\left(500 \mathrm{MHz}, \mathrm{CDCl}_{3}\right) \delta(\mathrm{ppm})=7.36-7.28(\mathrm{~m}, 2 \mathrm{H}), 7.28-7.21(\mathrm{~m}, 1 \mathrm{H})$, 7.20-7.14 (m, 2H), $5.73(\mathrm{dp}, J=29.5,7.6 \mathrm{~Hz}, 1 \mathrm{H}), 2.88(\mathrm{t}, J=7.7 \mathrm{~Hz}, 2 \mathrm{H}), 2.54(\mathrm{dt}, J=$ $16.5,7.8 \mathrm{~Hz}, 2 \mathrm{H}) ;{ }^{13} \mathrm{C} \mathrm{NMR}\left(126 \mathrm{MHz}, \mathrm{CDCl}_{3}\right) \delta(\mathrm{ppm})=163.0-160.2(\mathrm{~m}), 138.9,128.7$, $128.3,126.8,119.2\left(\mathrm{td}, J_{C-F}=22.2,9.4 \mathrm{~Hz}\right), 33.7\left(\mathrm{~d}, J_{C-F}=24.2 \mathrm{~Hz}\right), 31.6 ;{ }^{19} \mathrm{~F}$ NMR $(470$ $\left.\mathrm{MHz}, \mathrm{CDCl}_{3}\right) \delta(\mathrm{ppm})=83.3-80.7(\mathrm{~m}, 1 \mathrm{~F}), 69.6(\mathrm{ddd}, J=152.1,21.9,7.8 \mathrm{~Hz}, 4 \mathrm{~F}),-85.9$ (m, 1F); GC-MS (EI) m/z calcd for $\mathrm{C}_{10} \mathrm{H}_{11} \mathrm{~F}$ [M-SF $]^{+}$149.08; found 149.07. Under all other conditions tested for MS analysis (HRMS-ESI(+). HRMS-ESI(-), HRMS-APPI and GC-MS-CI), we never could detect any significant ions. The identification of the regioisomer was identified by the coupling constant between the vinylic proton, the vinylic fluorine and the pentafluorosulfanyl. The $\mathrm{Z}$ stereoisomer is established by the coupling constant between the vinylic proton and fluorine $(29.5 \mathrm{~Hz}=$ trans coupling $){ }^{22}$

\section{NMR spectra}




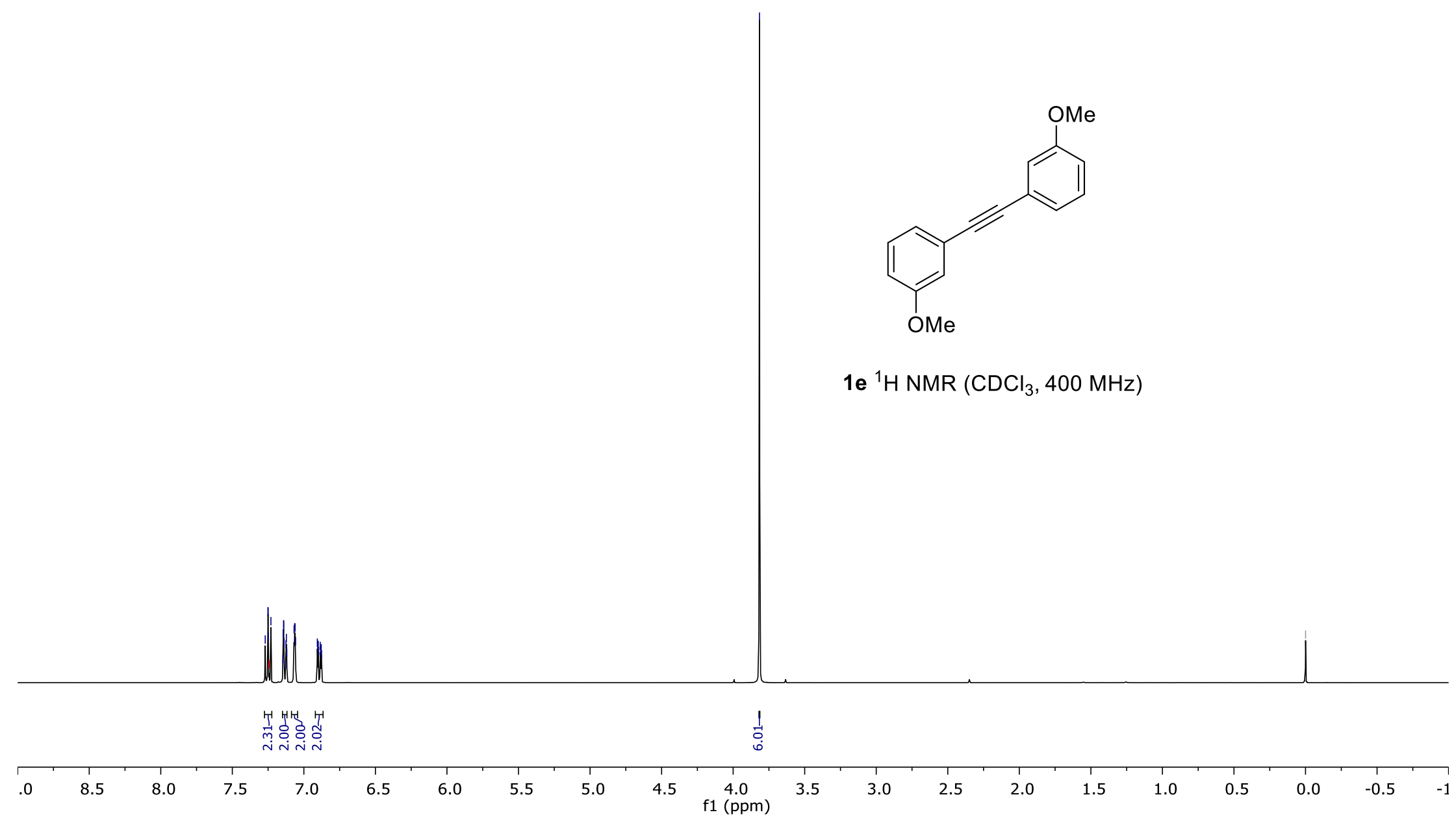




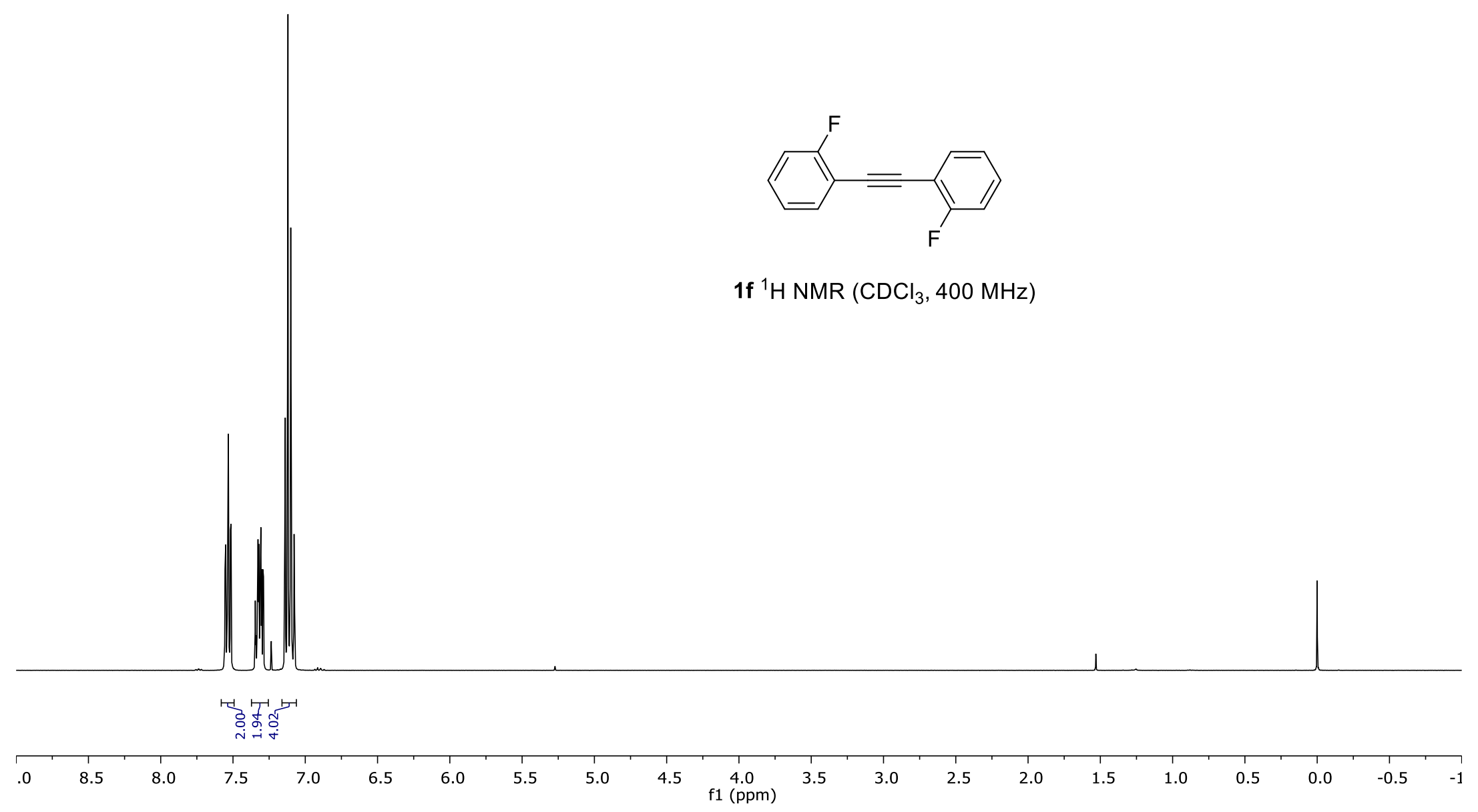




\begin{tabular}{|c|c|c|}
\hline 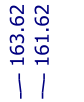 & 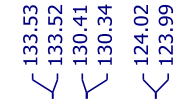 & 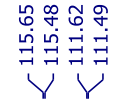 \\
\hline
\end{tabular}
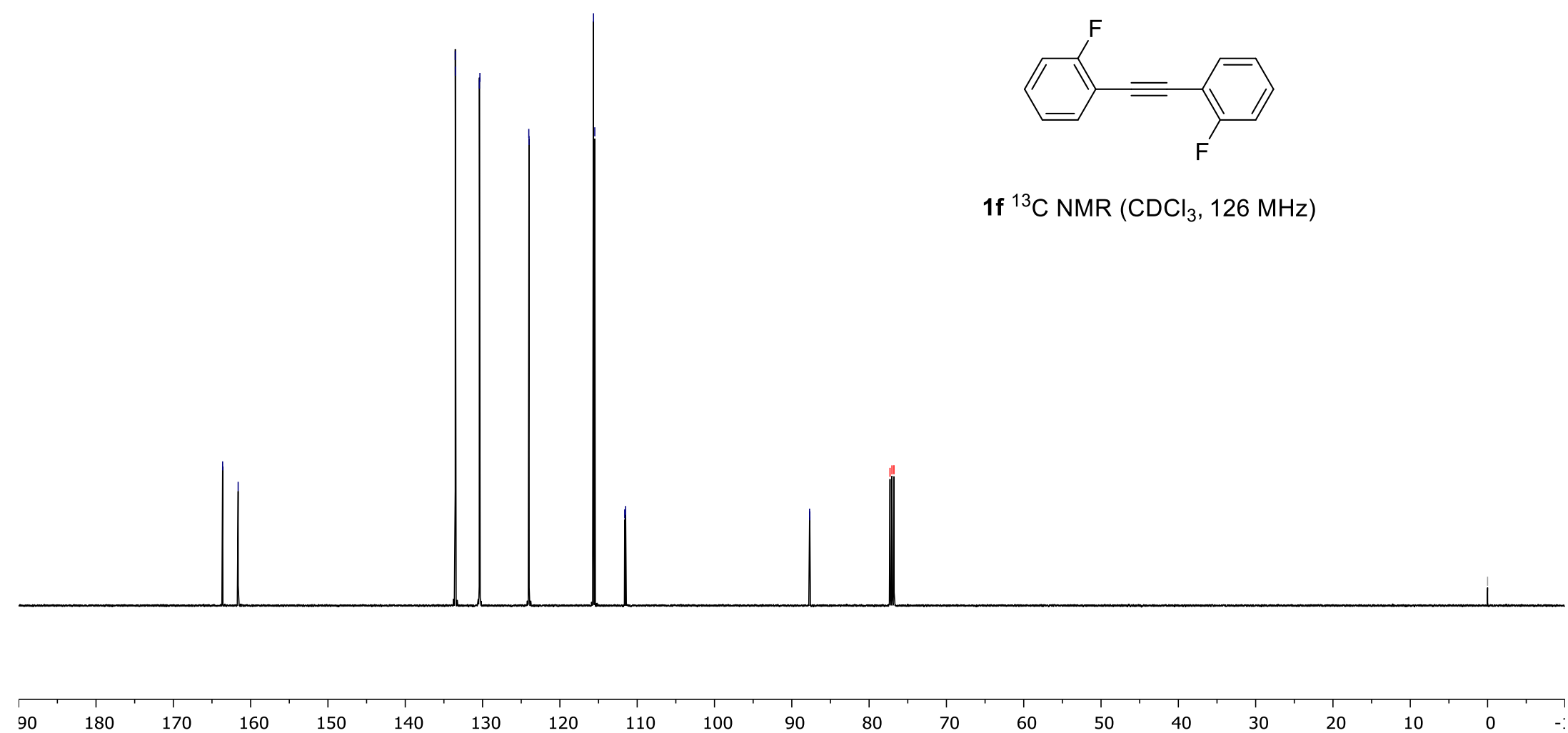

100

$\begin{array}{ll}90 & 1 \\ 90 & \end{array}$

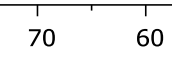

$60 \quad 50$ 


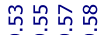



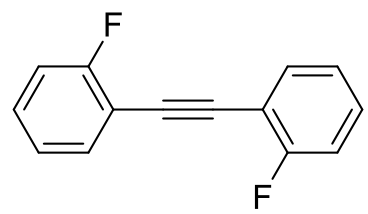

1f ${ }^{19} \mathrm{~F} \mathrm{NMR}\left(\mathrm{CDCl}_{3}, 470 \mathrm{MHz}\right)$

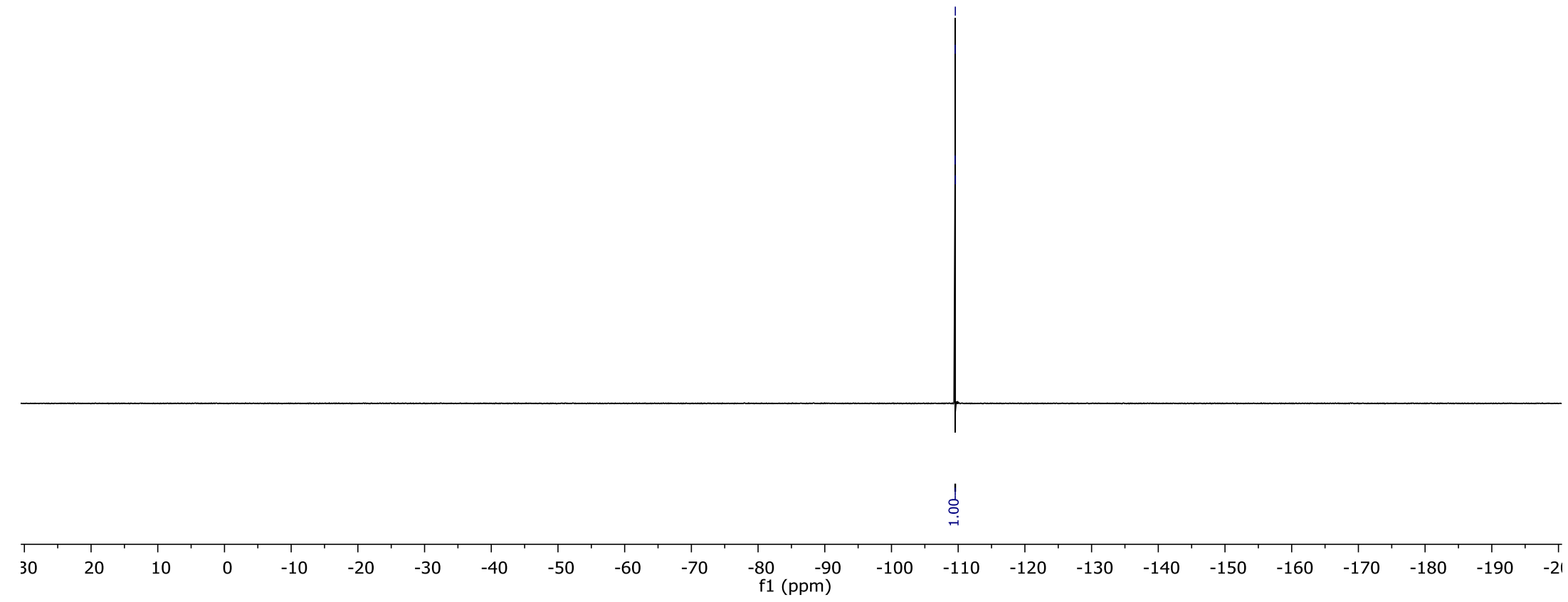


$\frac{\mathrm{m}}{\mathrm{u}}$

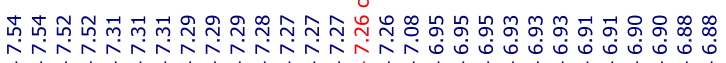

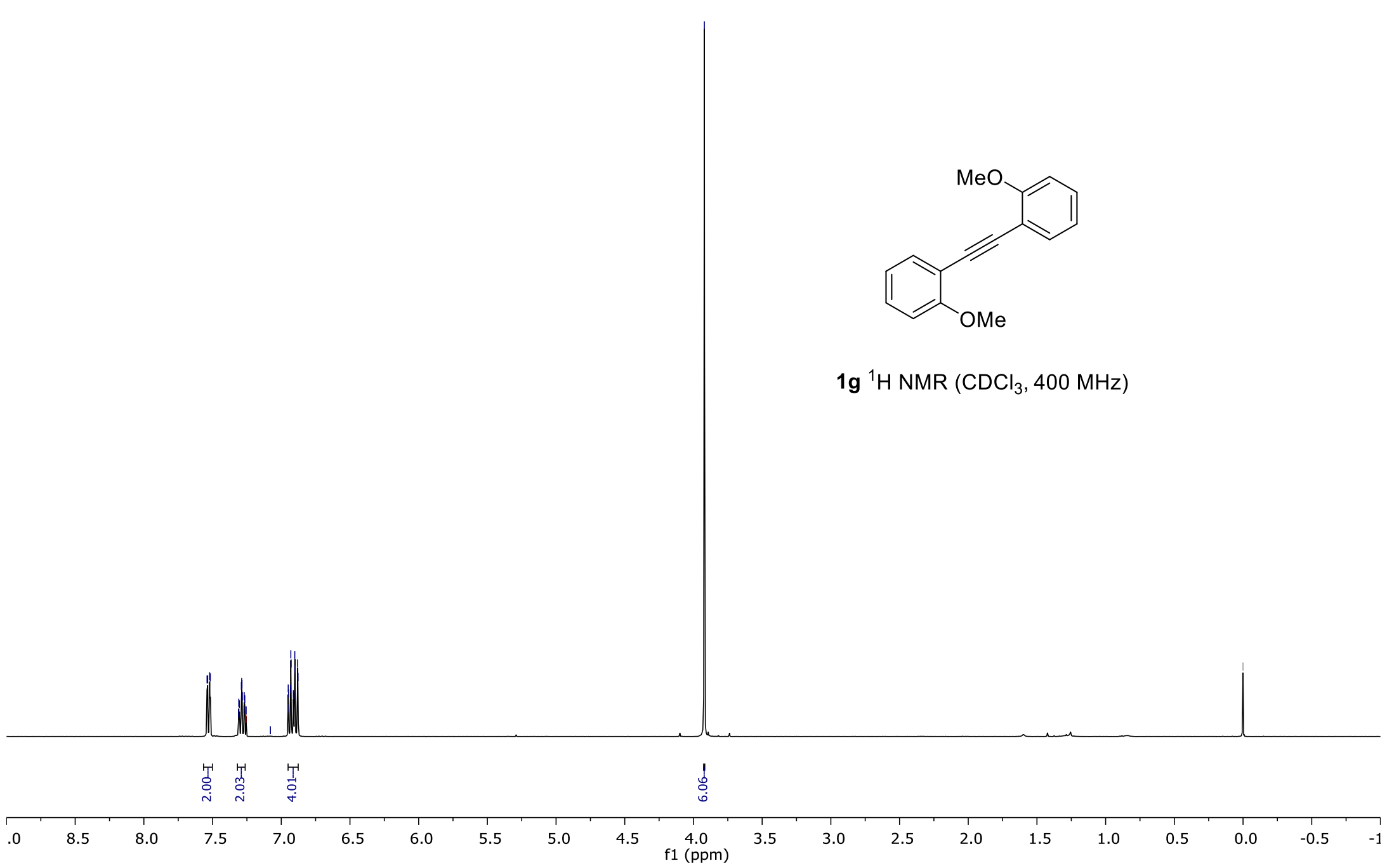




$$
\mathrm{Ph}=\mathrm{C}_{10} \mathrm{H}_{21}
$$

1k ${ }^{1} \mathrm{H}$ NMR $\left(\mathrm{CDCl}_{3}, 400 \mathrm{MHz}\right)$

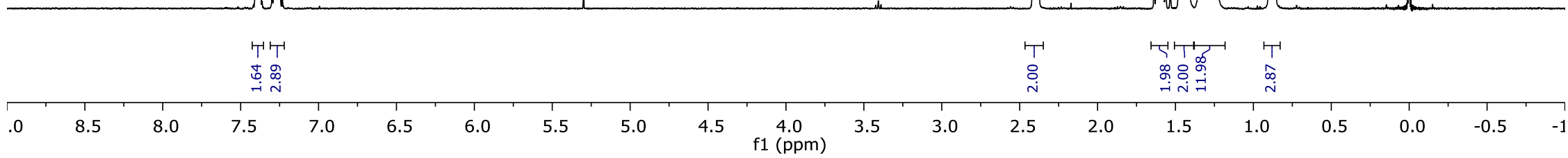




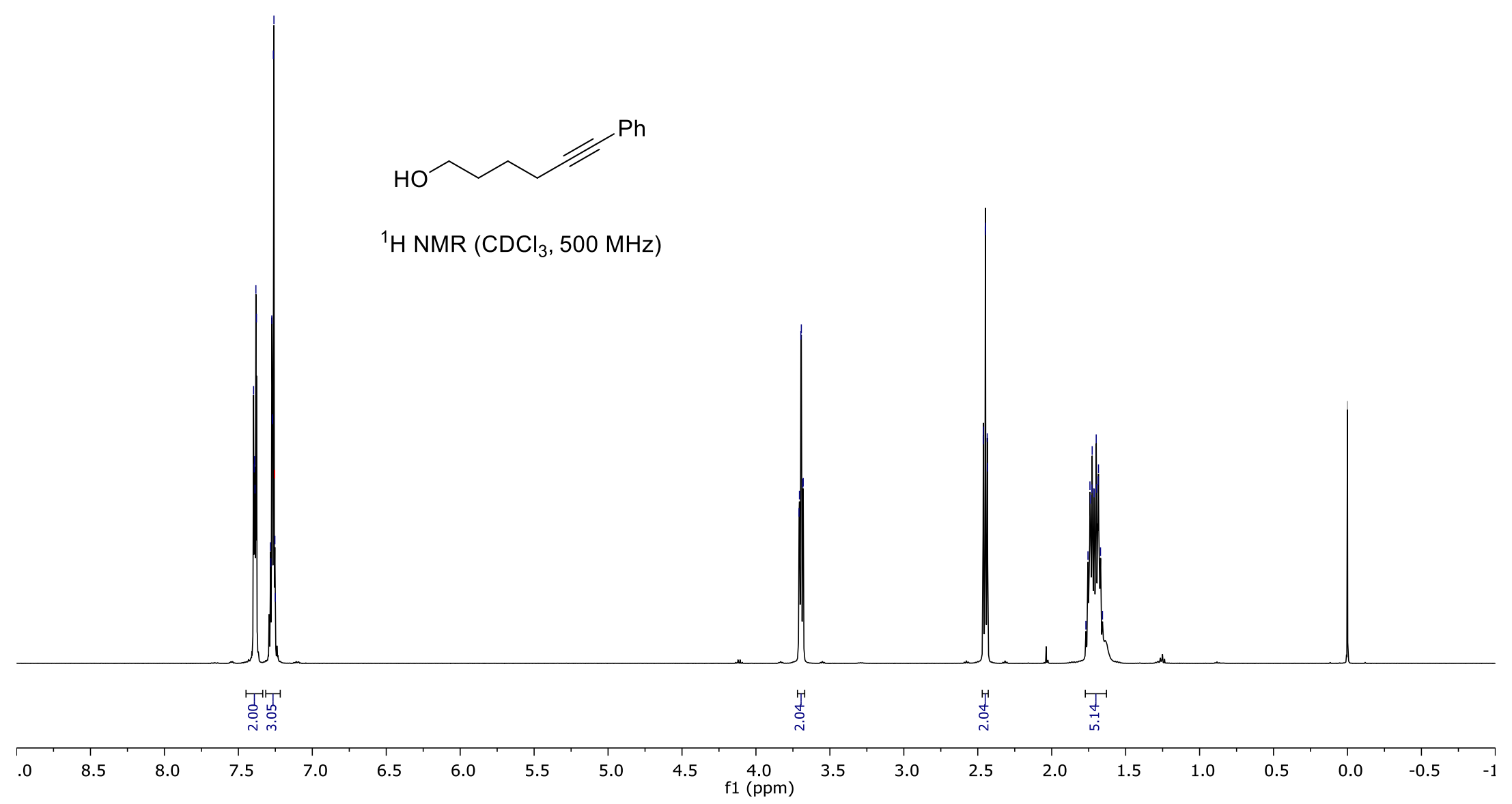




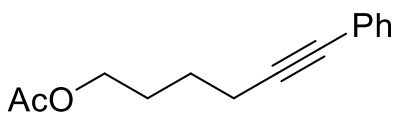

1/ ${ }^{1} \mathrm{H}$ NMR $\left(\mathrm{CDCl}_{3}, 500 \mathrm{MHz}\right)$

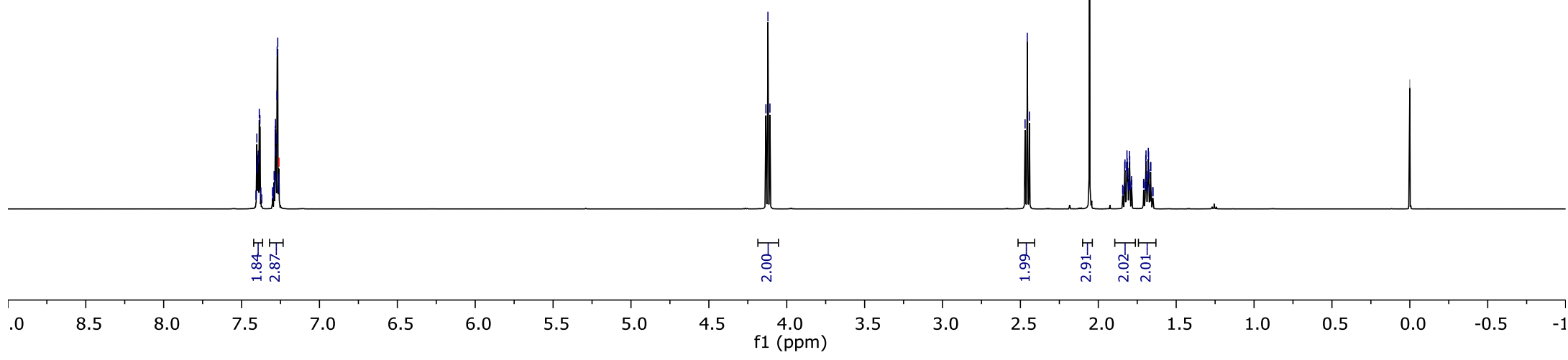



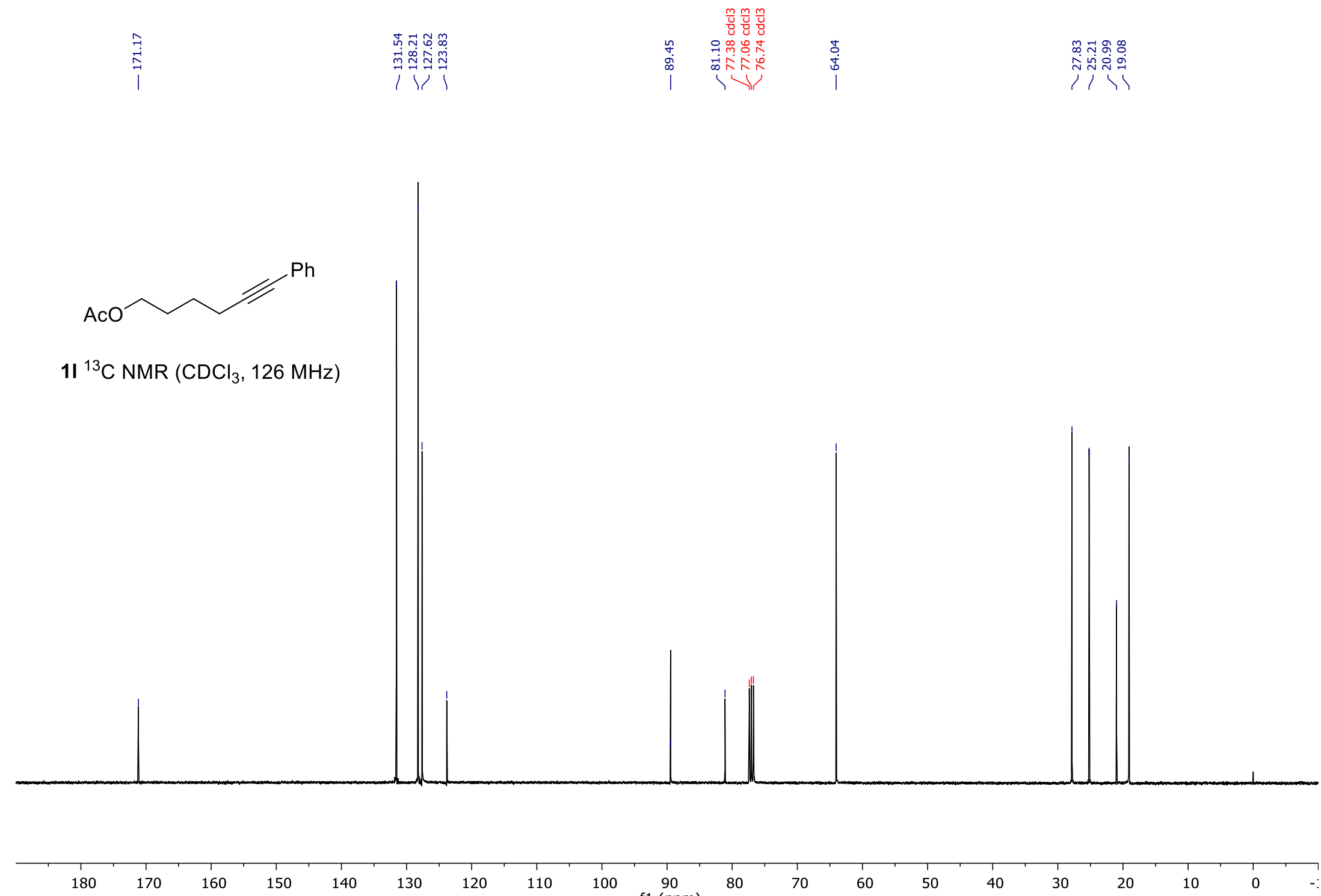
f1 (ppm)

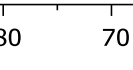

60

50 


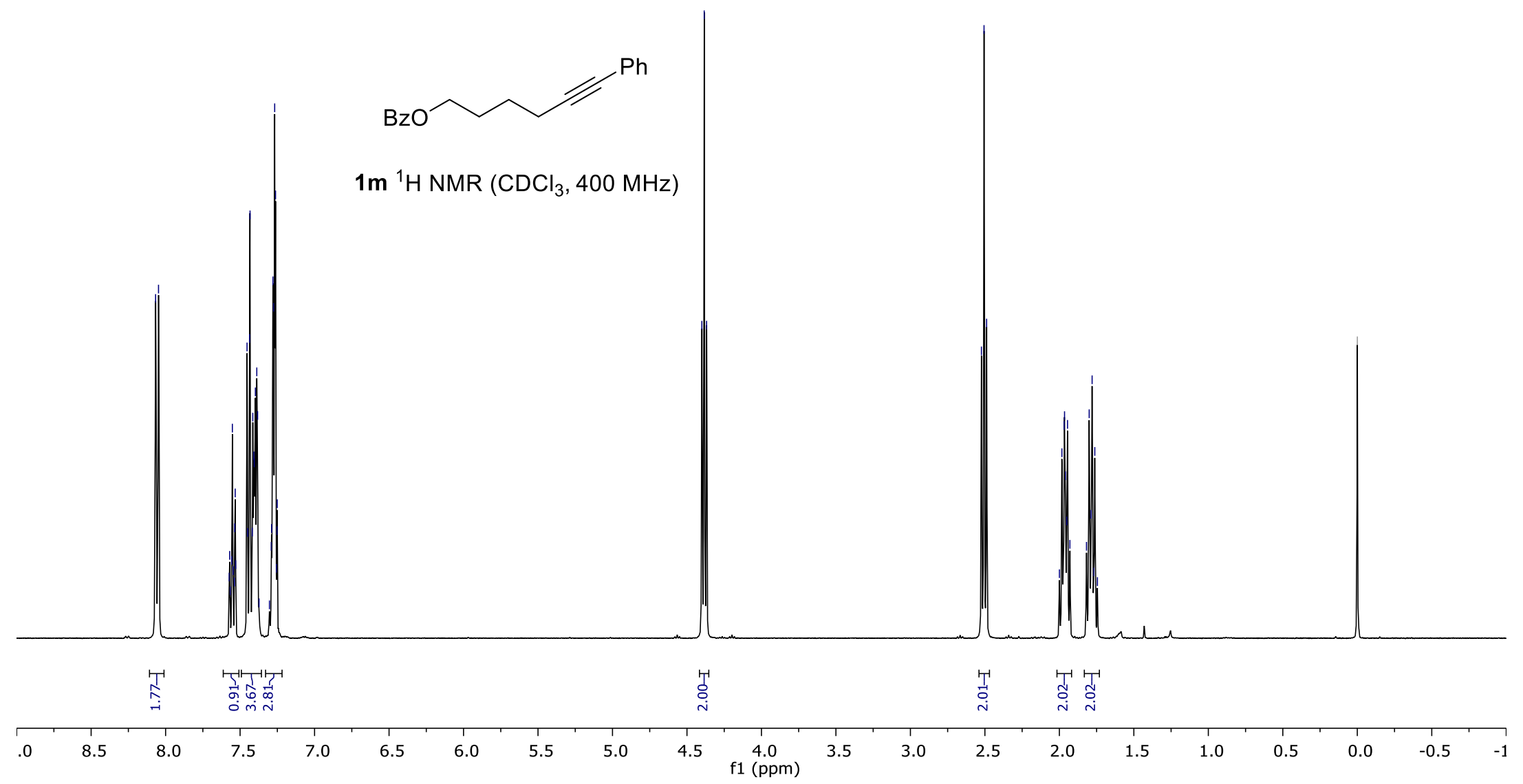



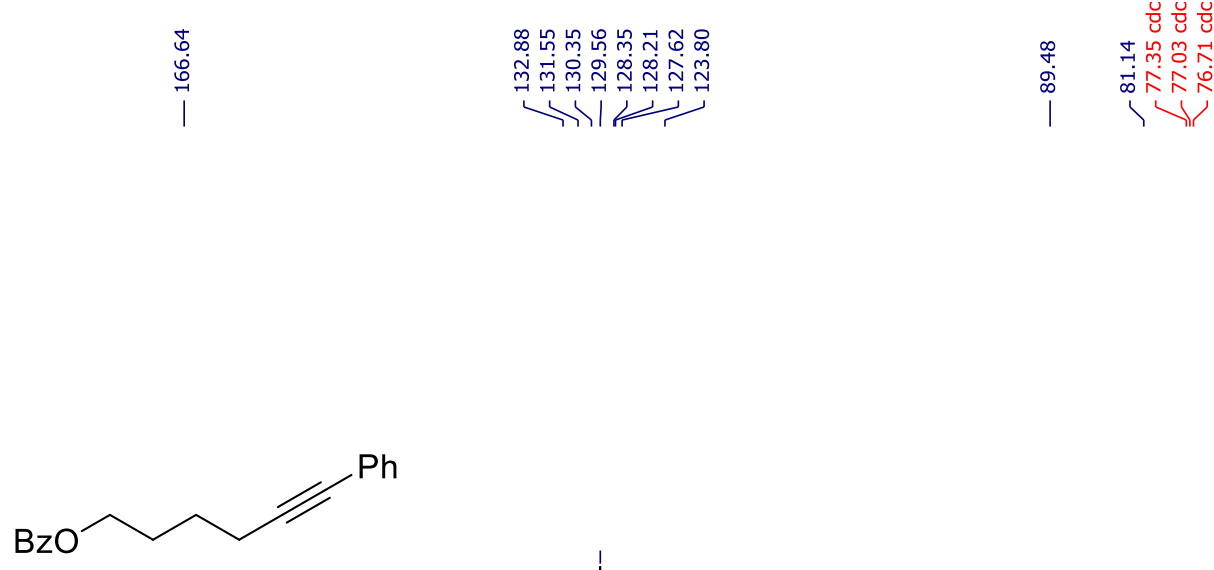

$1 \mathrm{~m}{ }^{13} \mathrm{C}$ NMR $\left(\mathrm{CDCl}_{3}, 101 \mathrm{MHz}\right)$
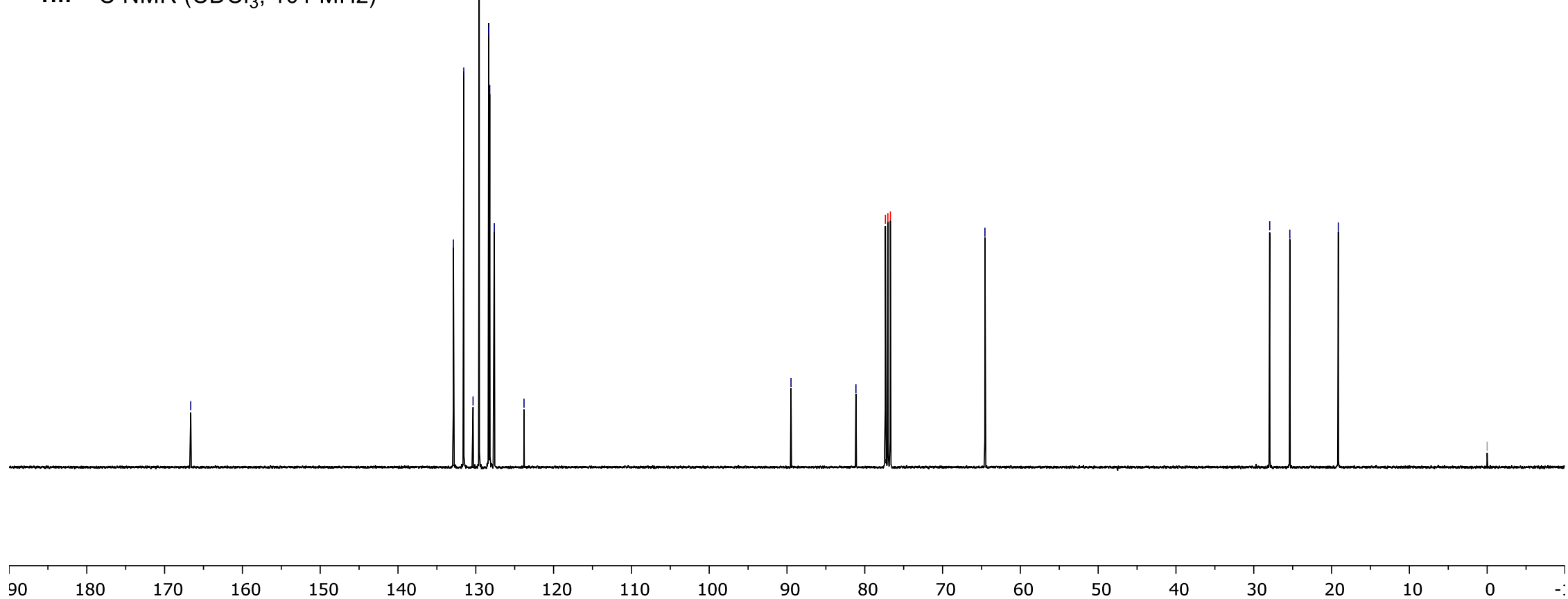


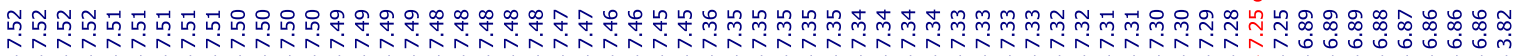

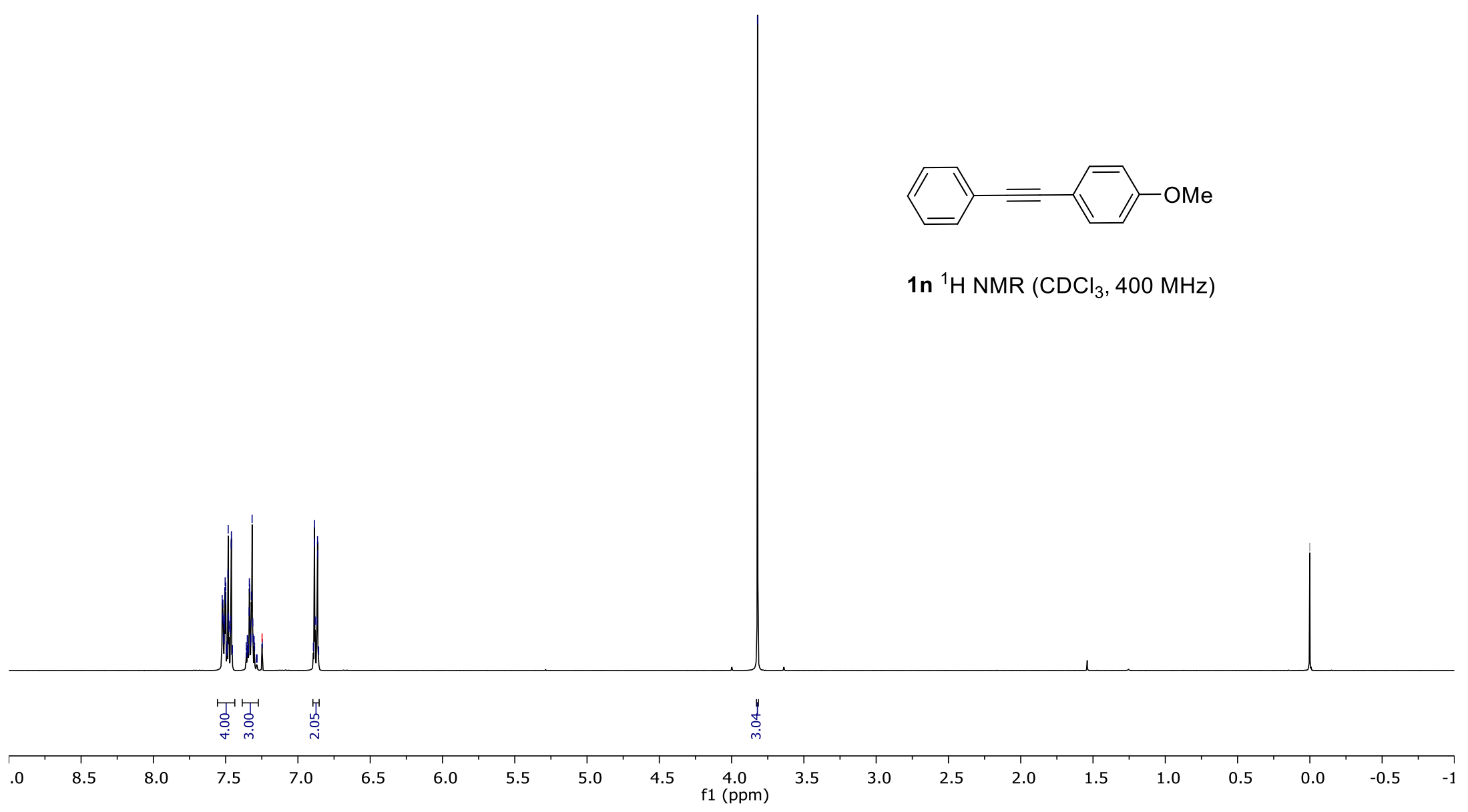




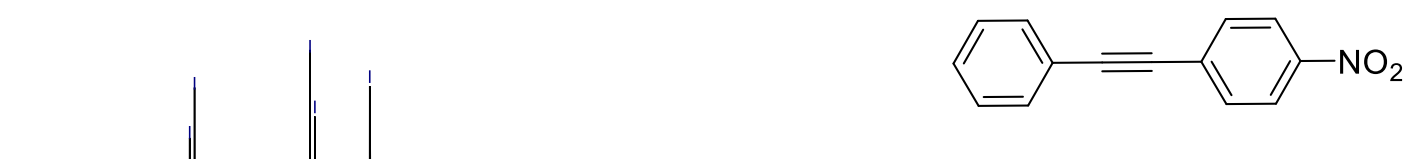

10 ${ }^{1} \mathrm{H} \mathrm{NMR}\left(\mathrm{CDCl}_{3}, 400 \mathrm{MHz}\right)$

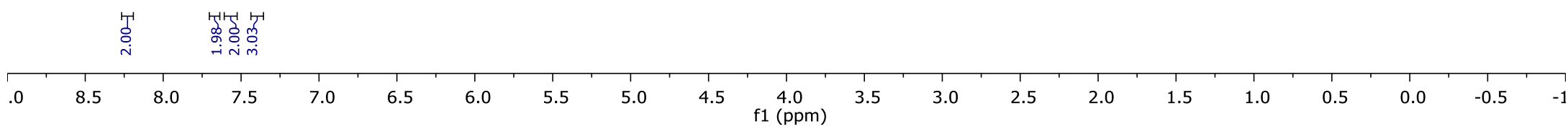




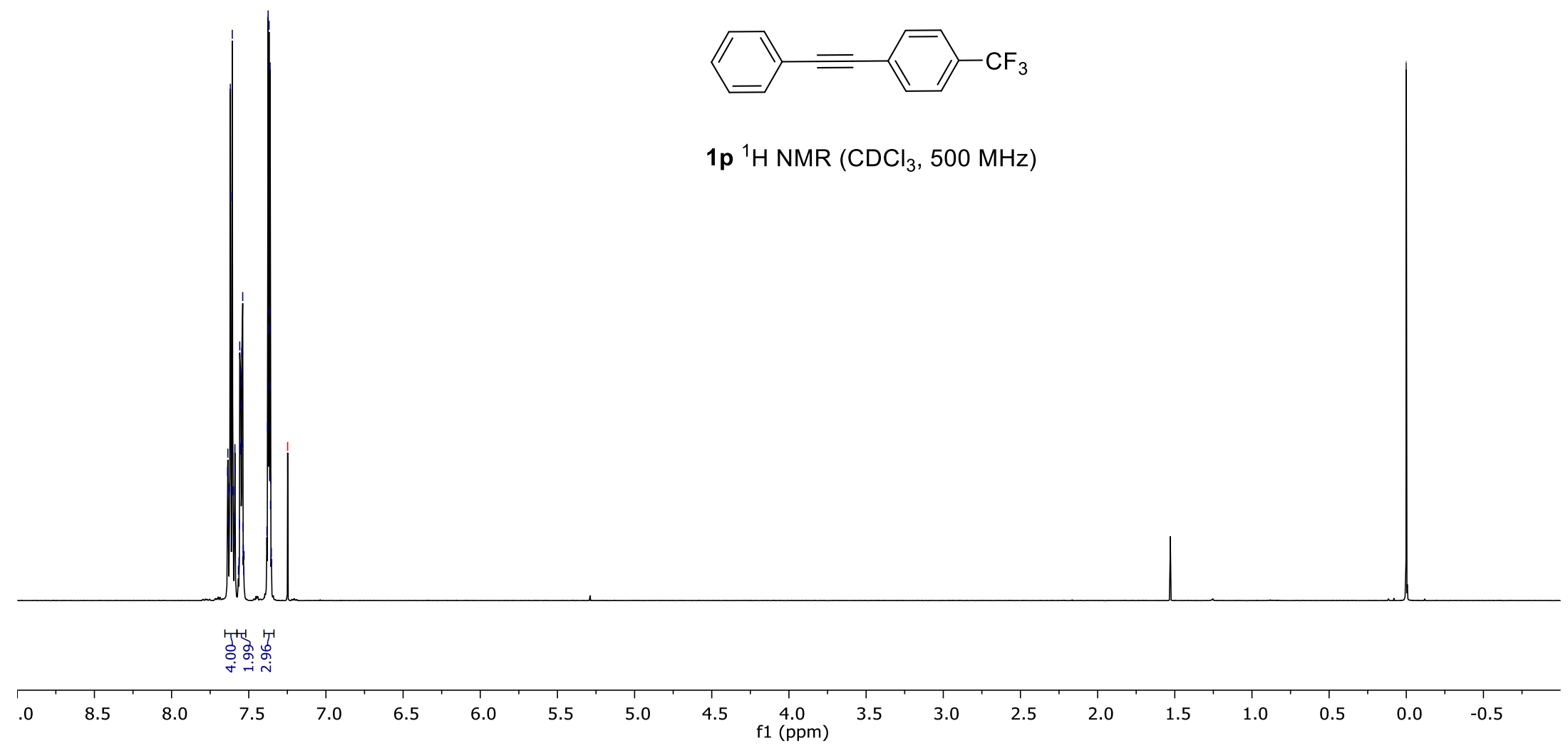




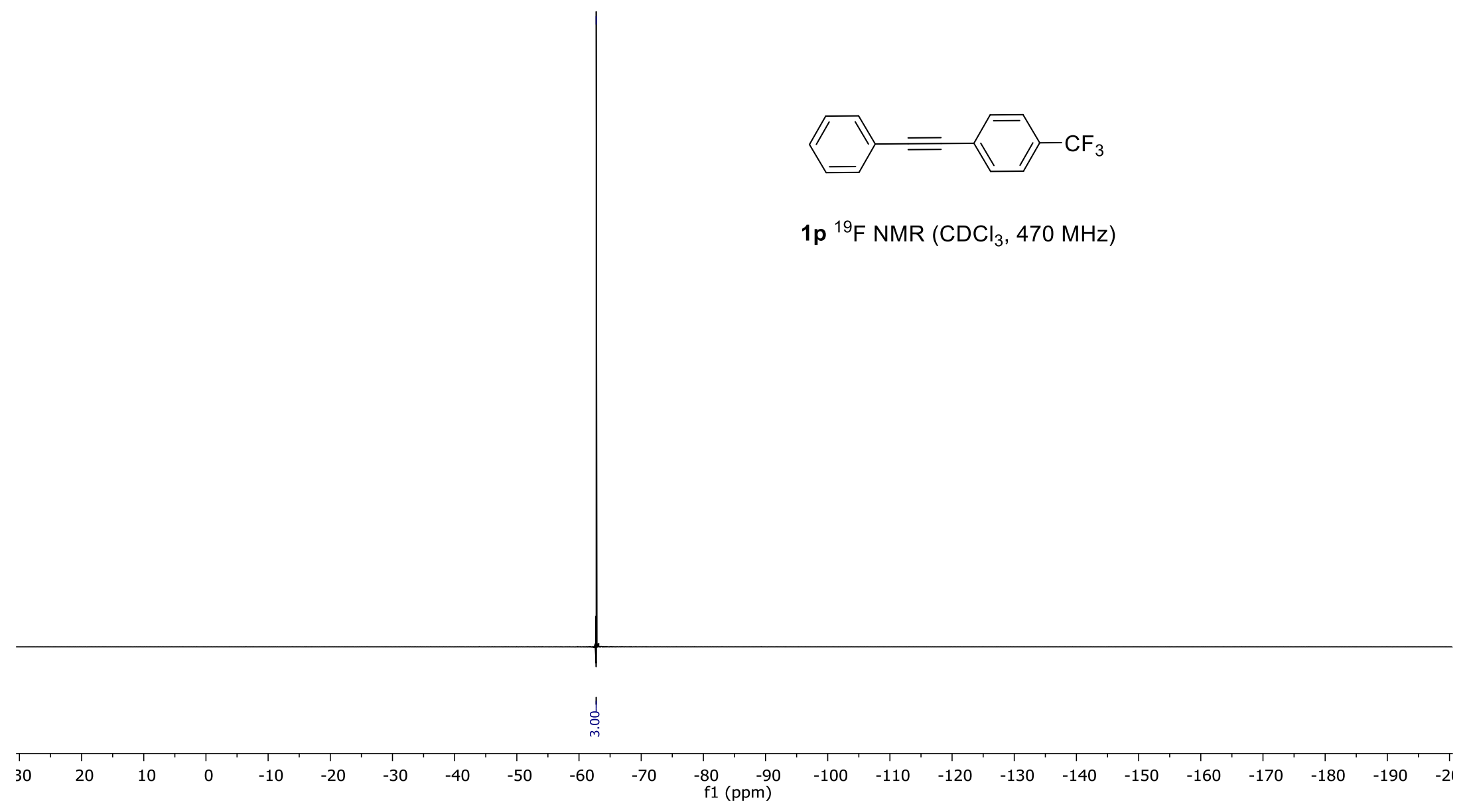




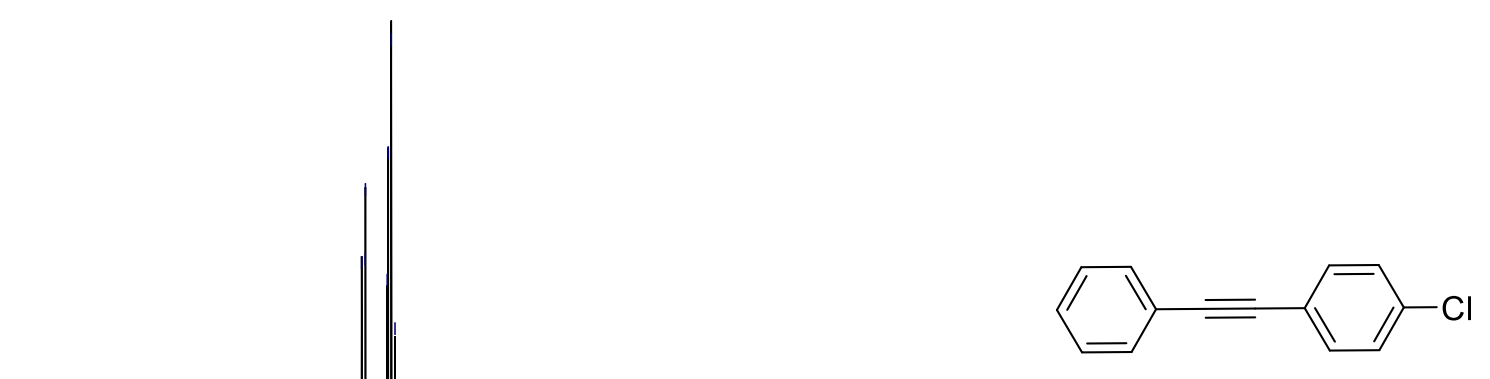

$1 \mathrm{q}^{1} \mathrm{H}$ NMR $\left(\mathrm{CDCl}_{3}, 500 \mathrm{MHz}\right)$

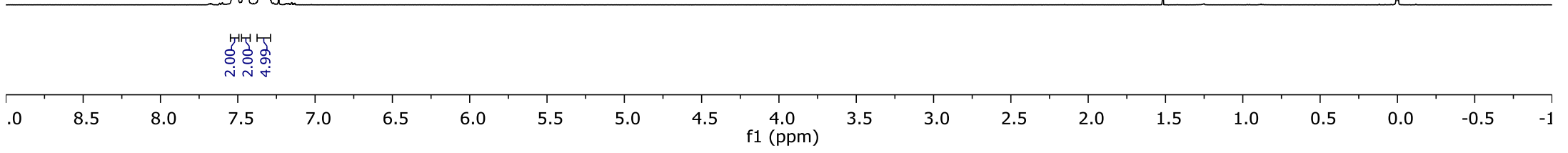




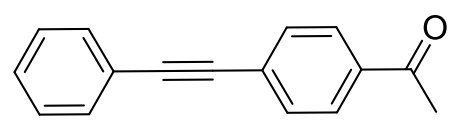

1r ${ }^{1} \mathrm{H}$ NMR $\left(\mathrm{CDCl}_{3}, 400 \mathrm{MHz}\right)$

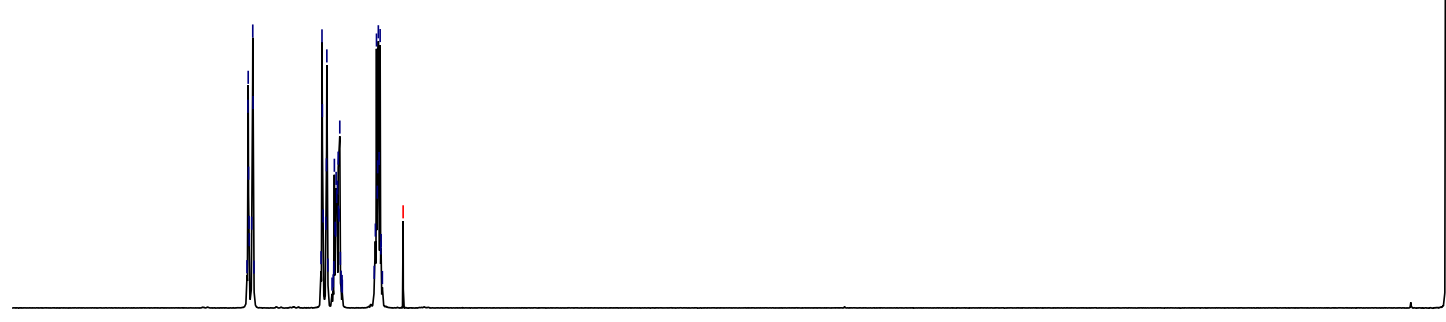

\begin{tabular}{|c|c|c|c|c|c|c|c|c|c|c|c|c|c|c|c|c|c|c|c|}
\hline & & 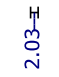 & 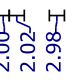 & & & & & & & & & & & & & & & & \\
\hline .0 & 8.5 & 8.0 & 7.5 & 7.0 & 6.5 & 6.0 & 5.5 & 5.0 & 4.5 & $\begin{array}{l}4.0 \\
(\mathrm{ppm})\end{array}$ & 3.5 & 3.0 & 2.5 & 2.0 & 1.5 & 1.0 & 0.5 & 0.0 & -0.5 \\
\hline
\end{tabular}




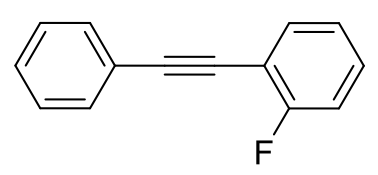

1s ${ }^{1} \mathrm{H}$ NMR $\left(\mathrm{CDCl}_{3}, 400 \mathrm{MHz}\right)$

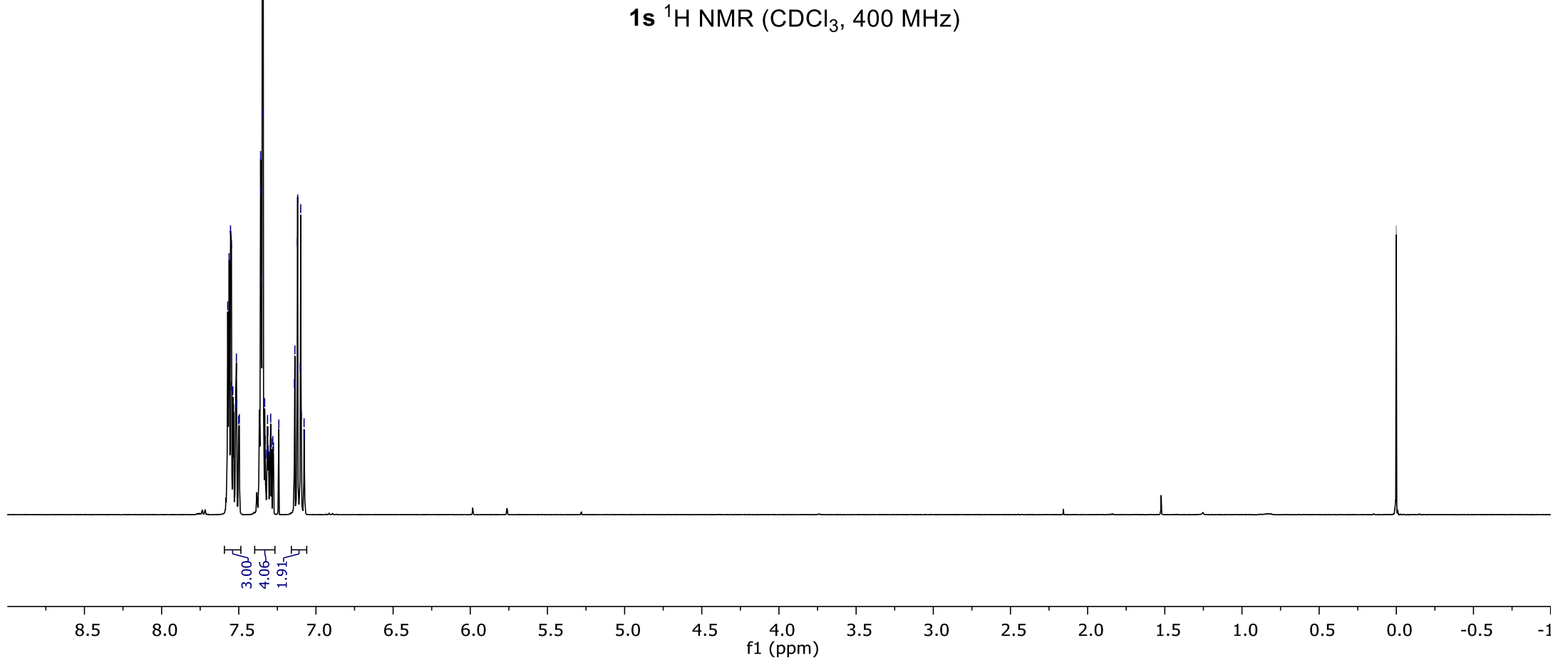




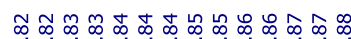

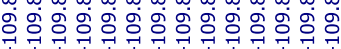

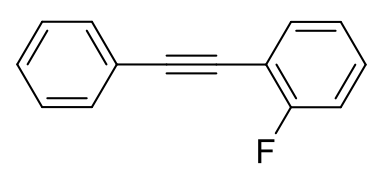

1s ${ }^{19} \mathrm{~F} \mathrm{NMR}\left(\mathrm{CDCl}_{3}, 376 \mathrm{MHz}\right)$

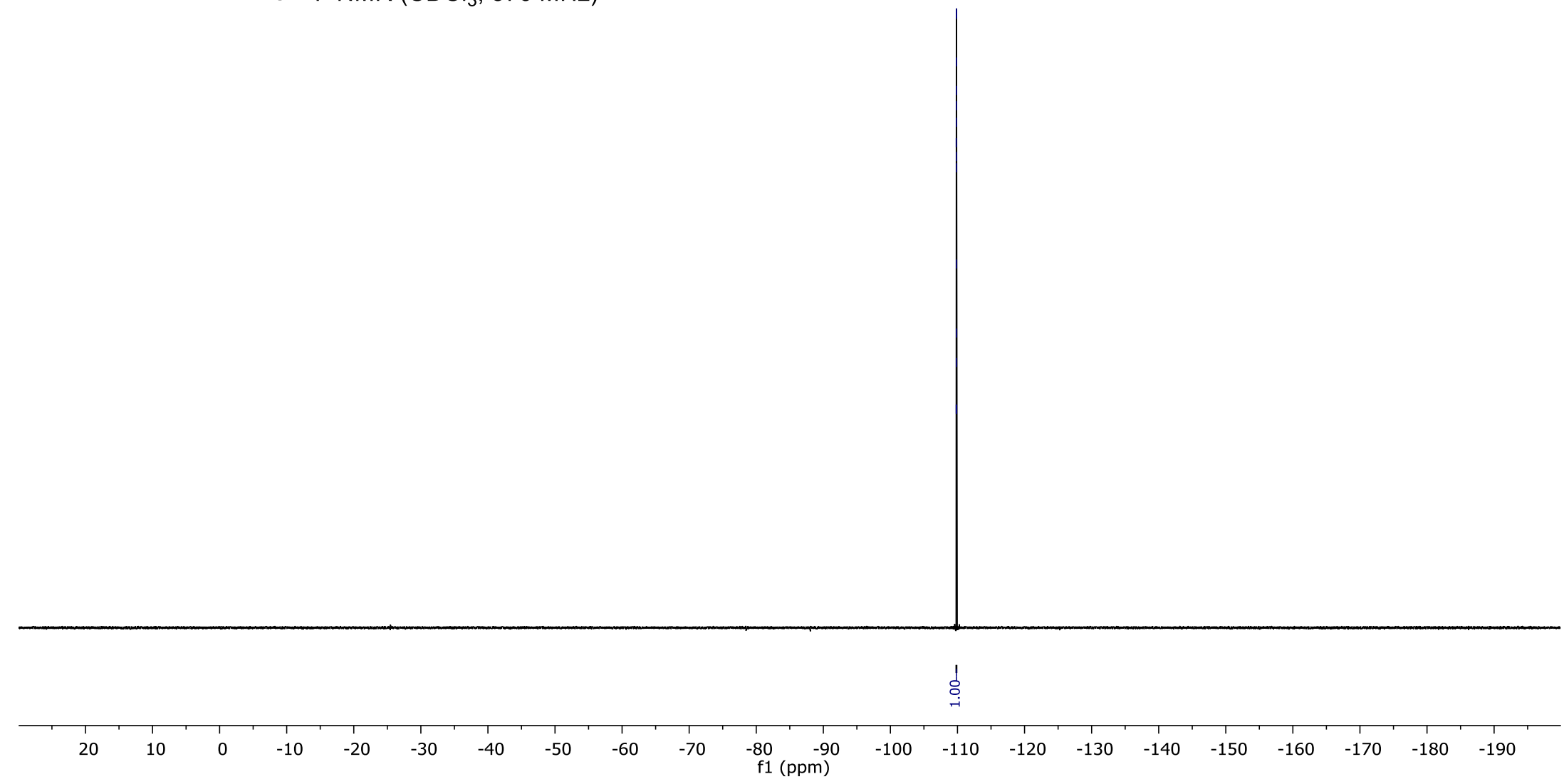




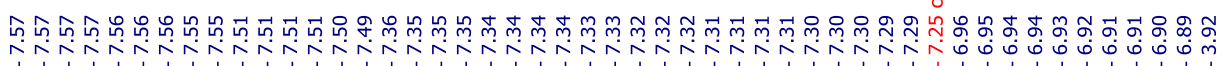

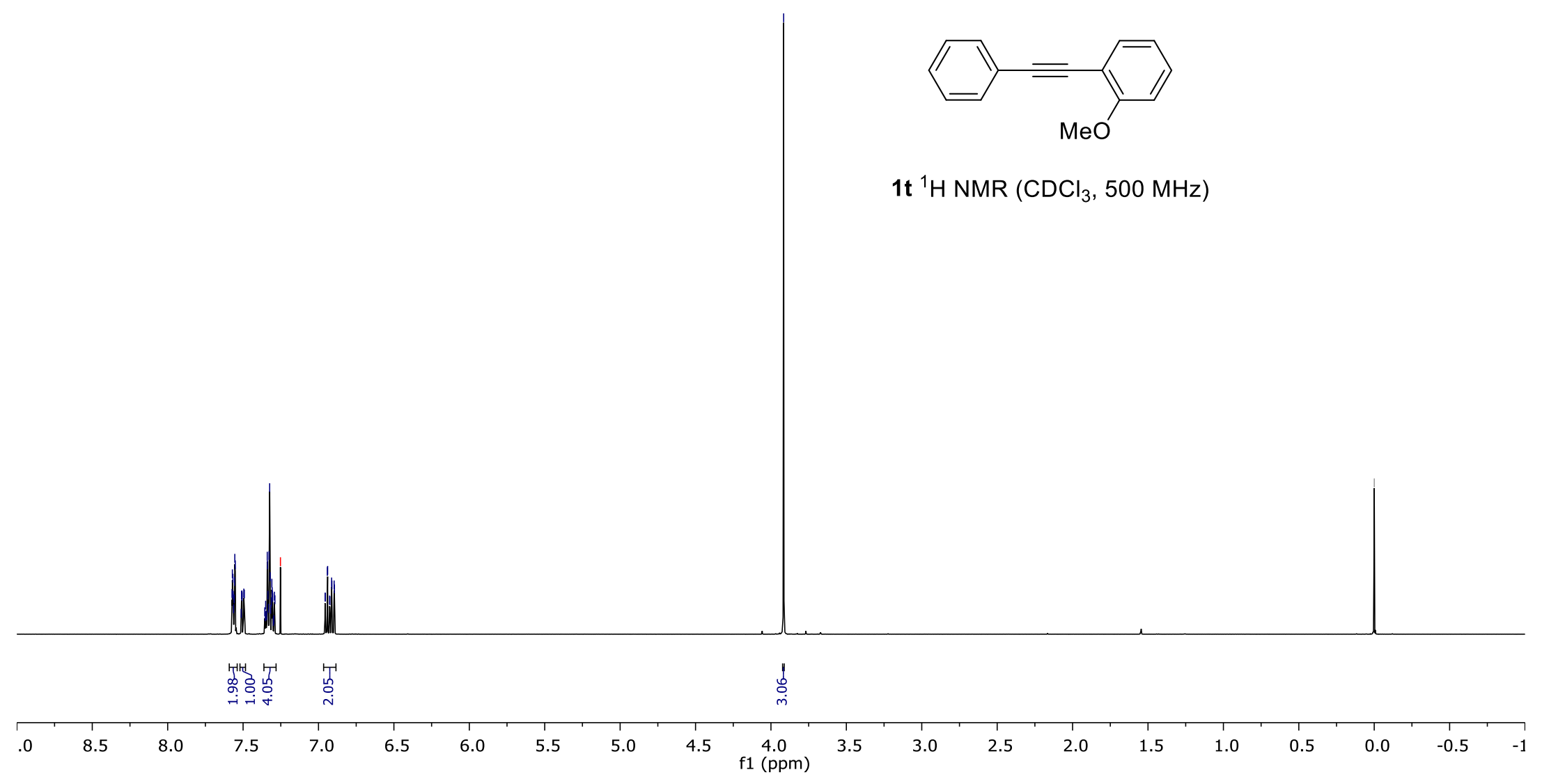




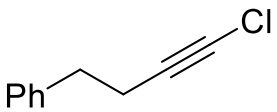

$1 \mathrm{u}^{1} \mathrm{H} \mathrm{NMR}\left(\mathrm{CDCl}_{3}, 500 \mathrm{MHz}\right)$

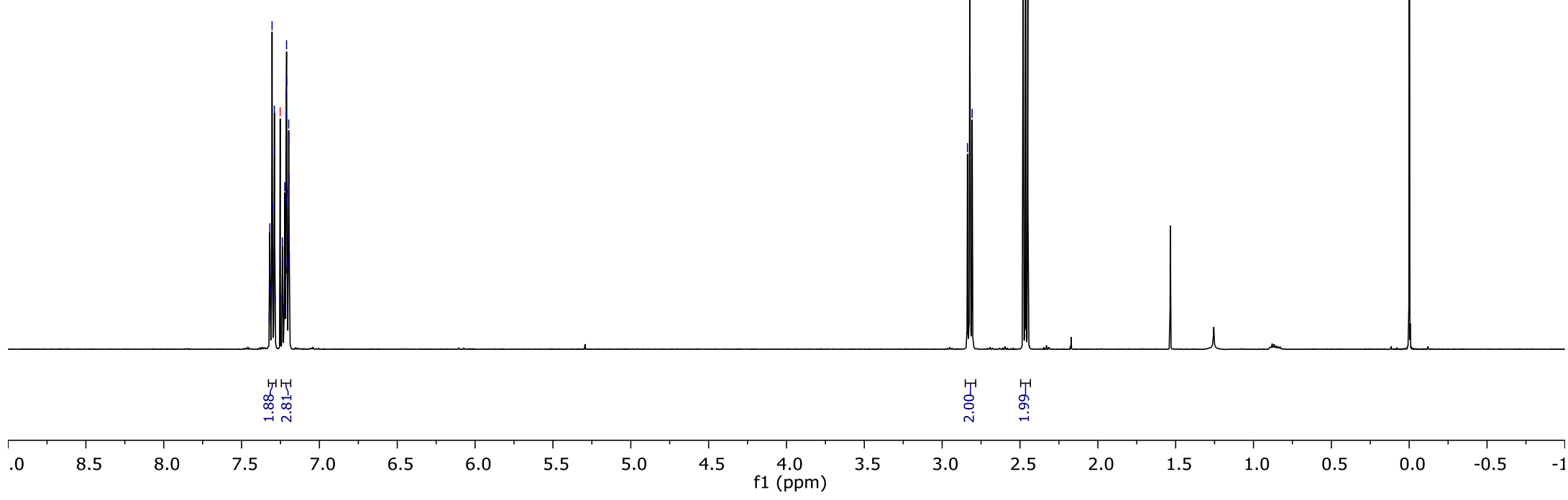




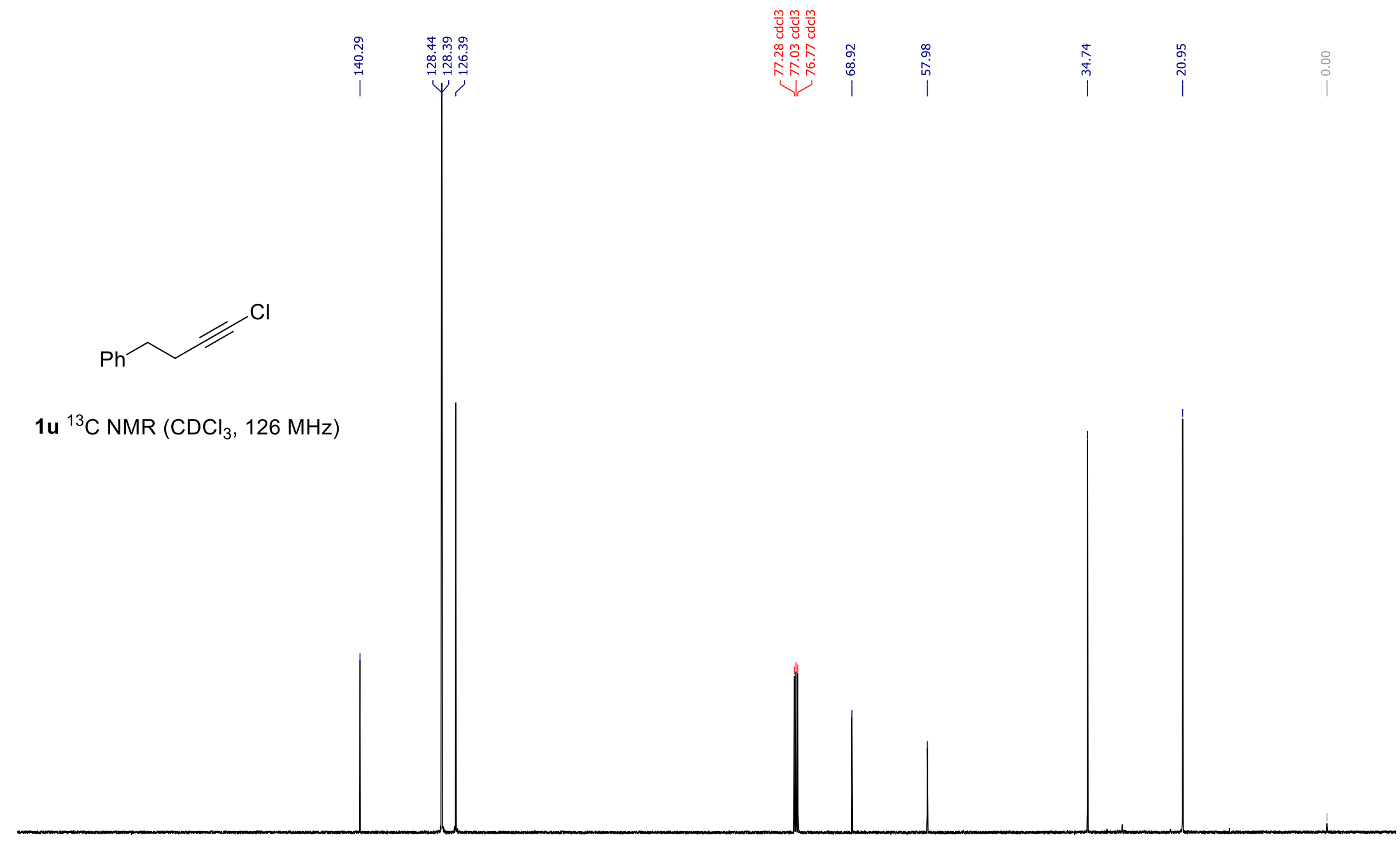$$
90
$$

18

170

160

150

140

130

120

110

100 90
$\mathrm{f} 1(\mathrm{ppm})$

$80 \quad 70$

60

50

40

30

10 0 


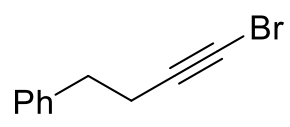

1v ${ }^{1} \mathrm{H} \mathrm{NMR}\left(\mathrm{CDCl}_{3}, 400 \mathrm{MHz}\right)$

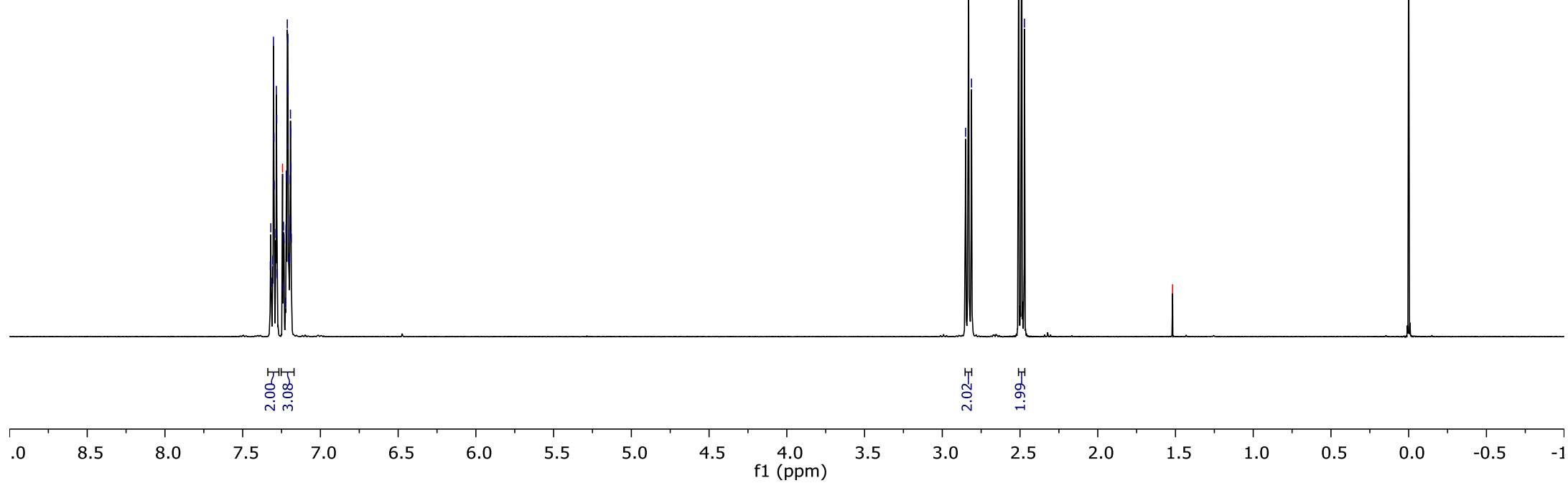




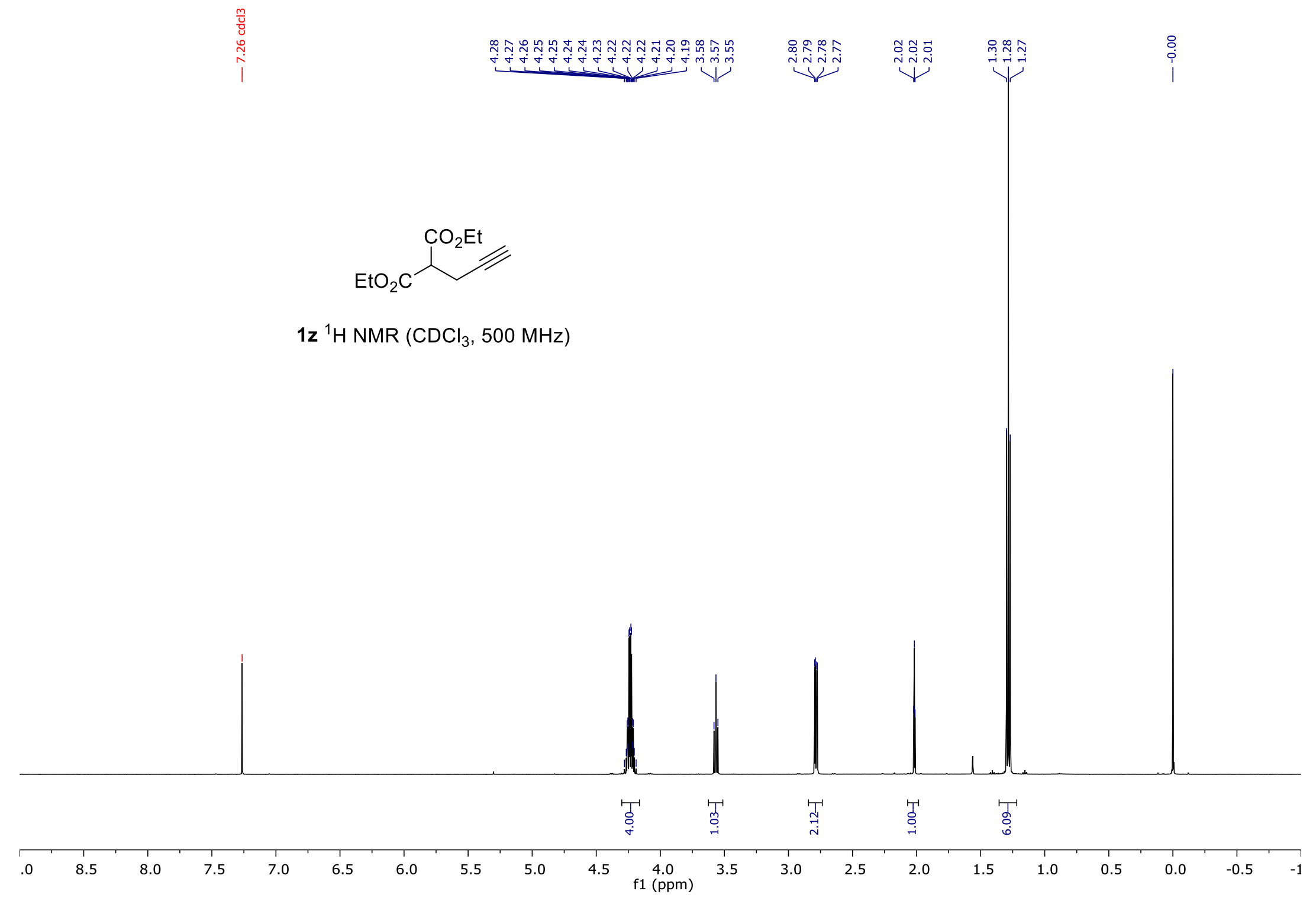




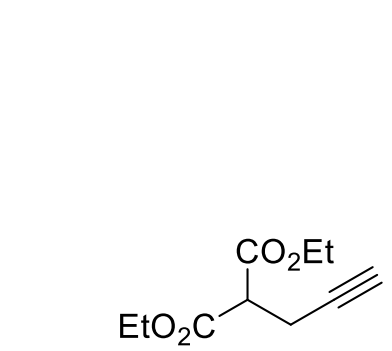

$1 \mathrm{z}^{13} \mathrm{C}$ NMR $\left(\mathrm{CDCl}_{3}, 126 \mathrm{MHz}\right)$

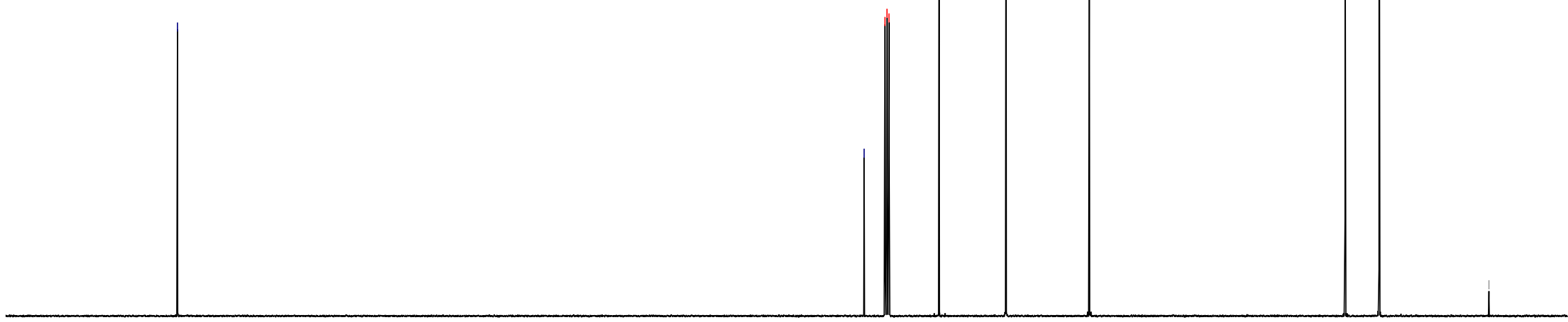




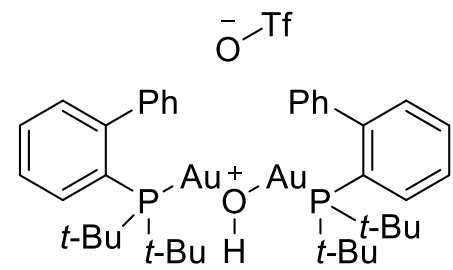

[\{Au(JohnPhos) $\left.\}_{2}(\mu \mathrm{OH})\right] \mathrm{OTf}{ }^{1} \mathrm{H}$ NMR $\left(\mathrm{CDCl}_{3}, 500 \mathrm{MHz}\right)$

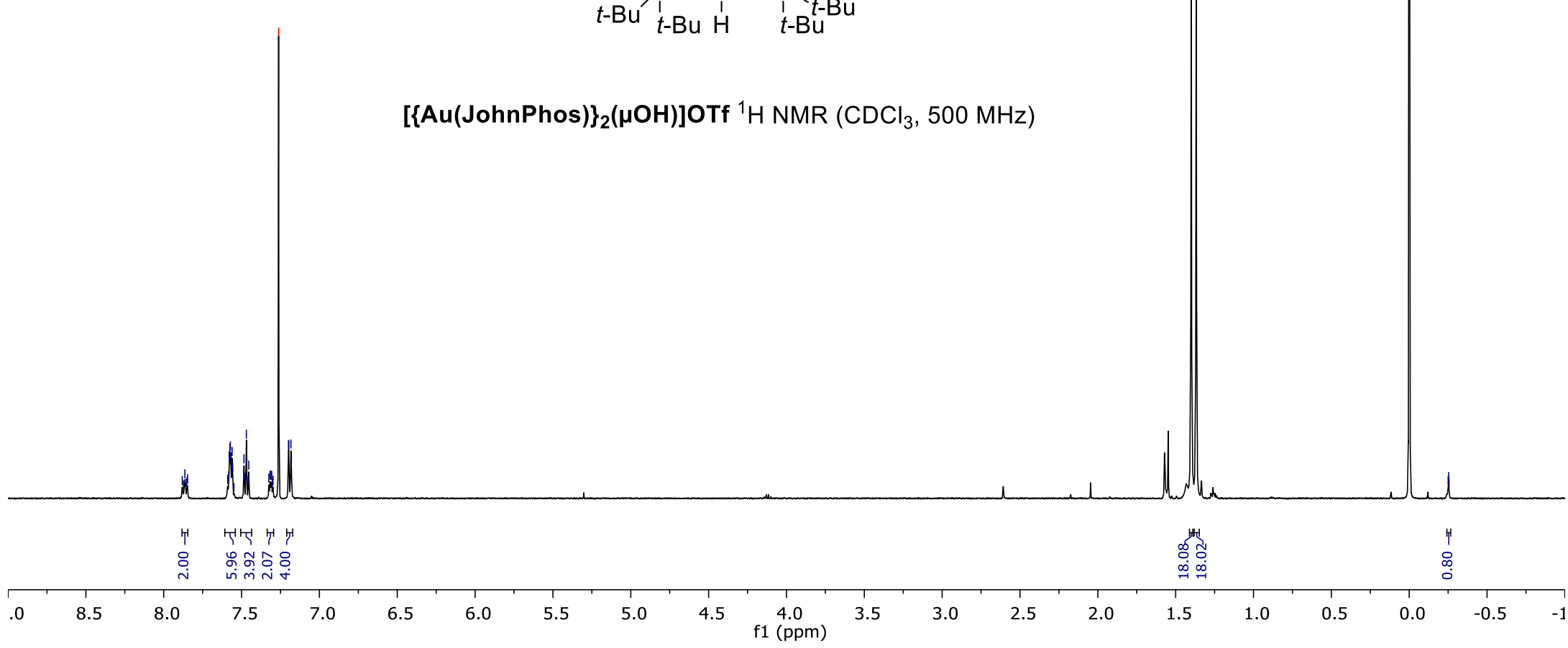


ว

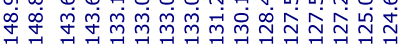

$\checkmark Y \longrightarrow 1 / 1$

$$
\bar{O}^{-T f}
$$

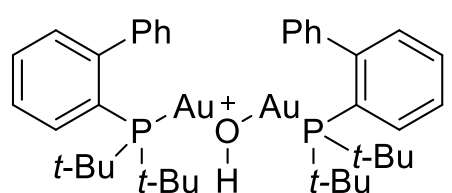

$\left[\{\mathrm{Au}(\mathrm{JohnPhos})\}_{2}(\mu \mathrm{OH})\right] \mathrm{OTf}{ }^{13} \mathrm{C} \mathrm{NMR}\left(\mathrm{CDCl}_{3}, 126 \mathrm{MHz}\right)$

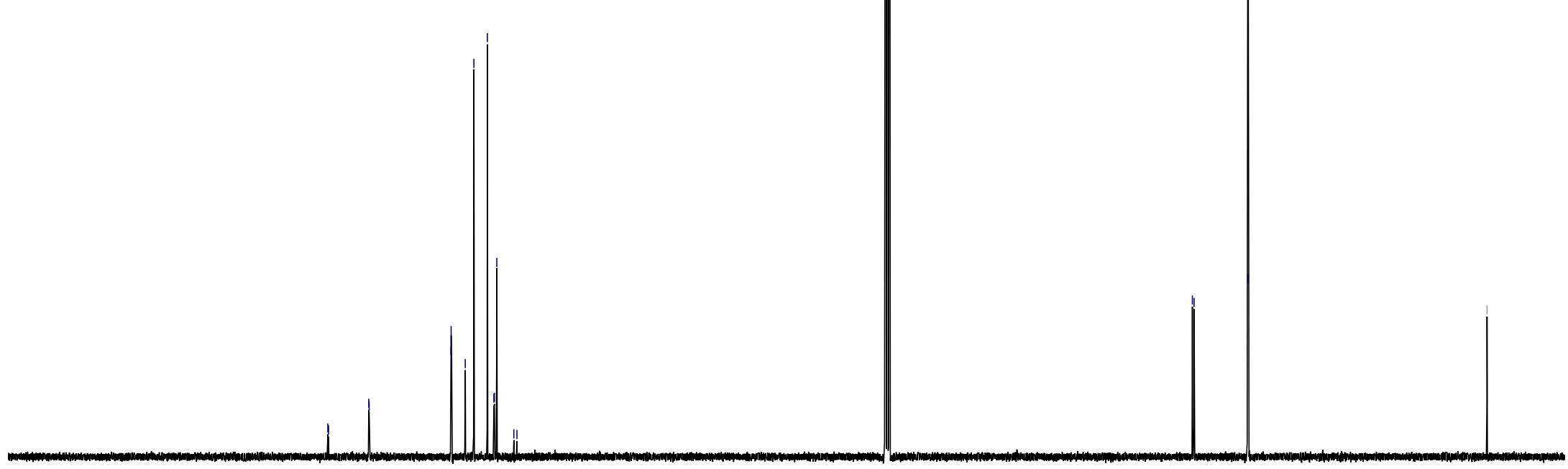




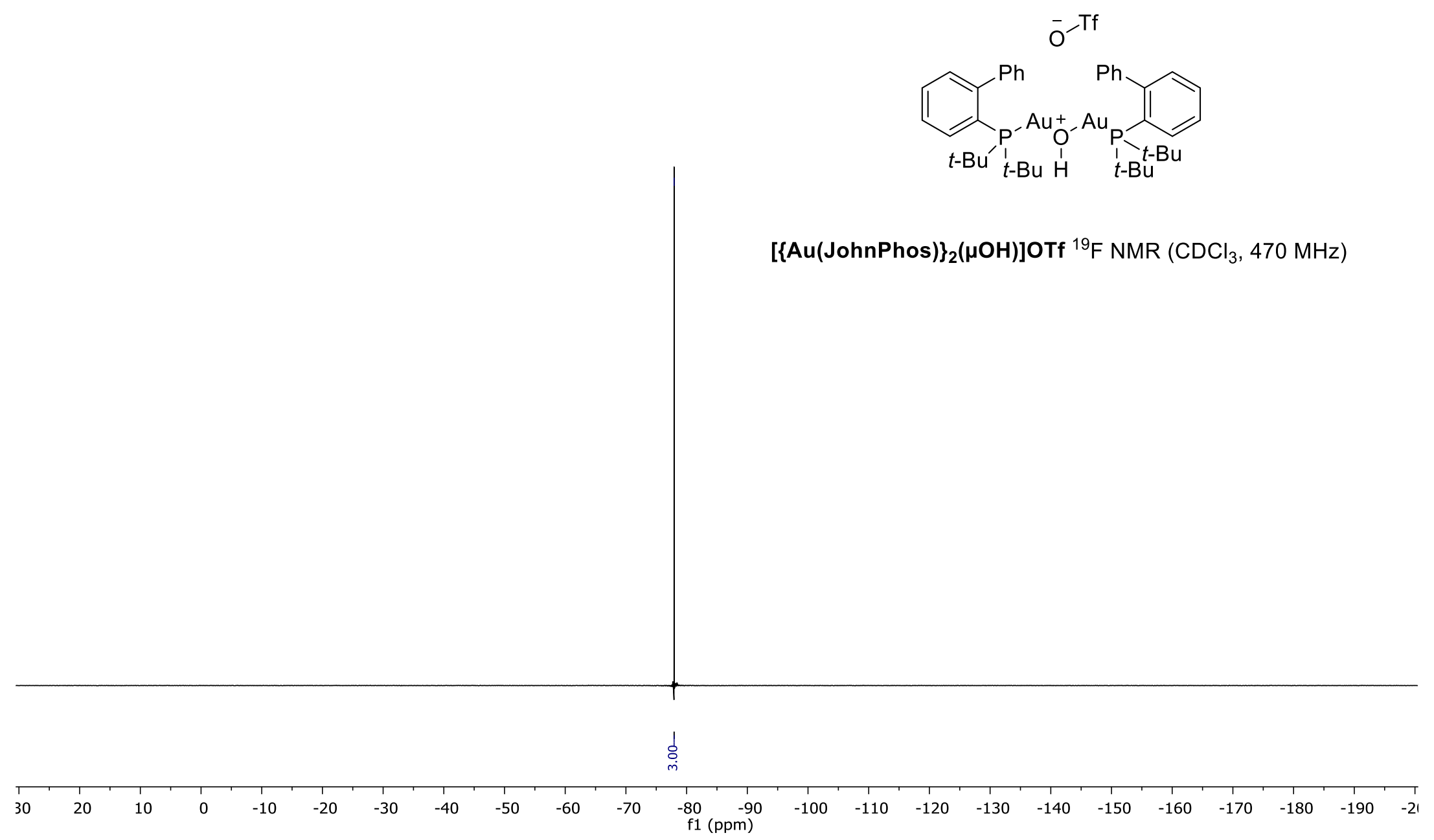




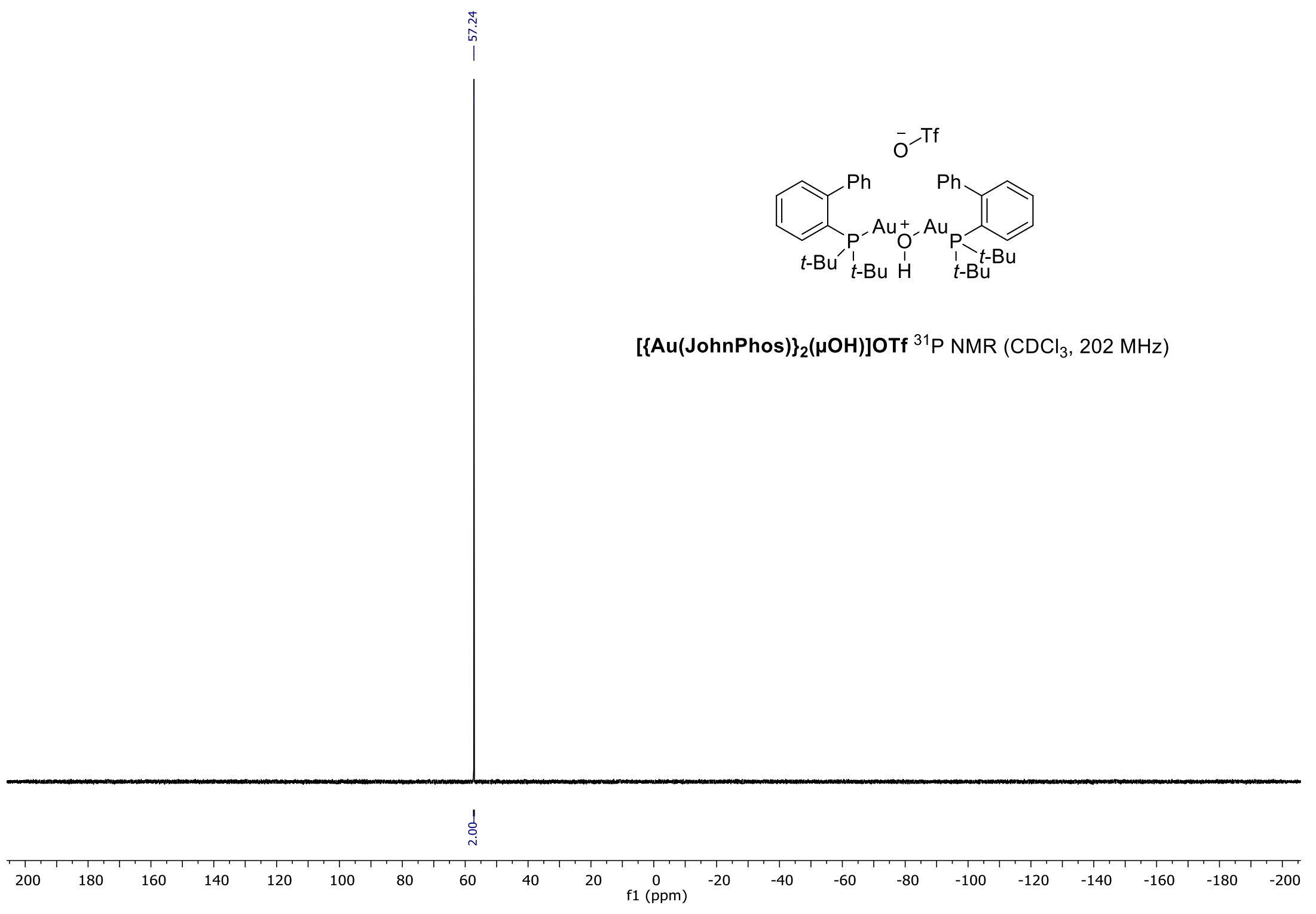




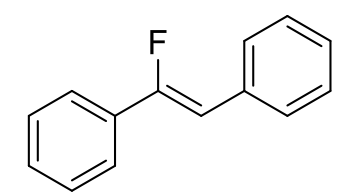

2a ${ }^{19} \mathrm{~F} \mathrm{NMR}\left(\mathrm{CDCl}_{3}, 470 \mathrm{MHz}\right)$

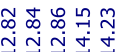

쿡구국

Vij

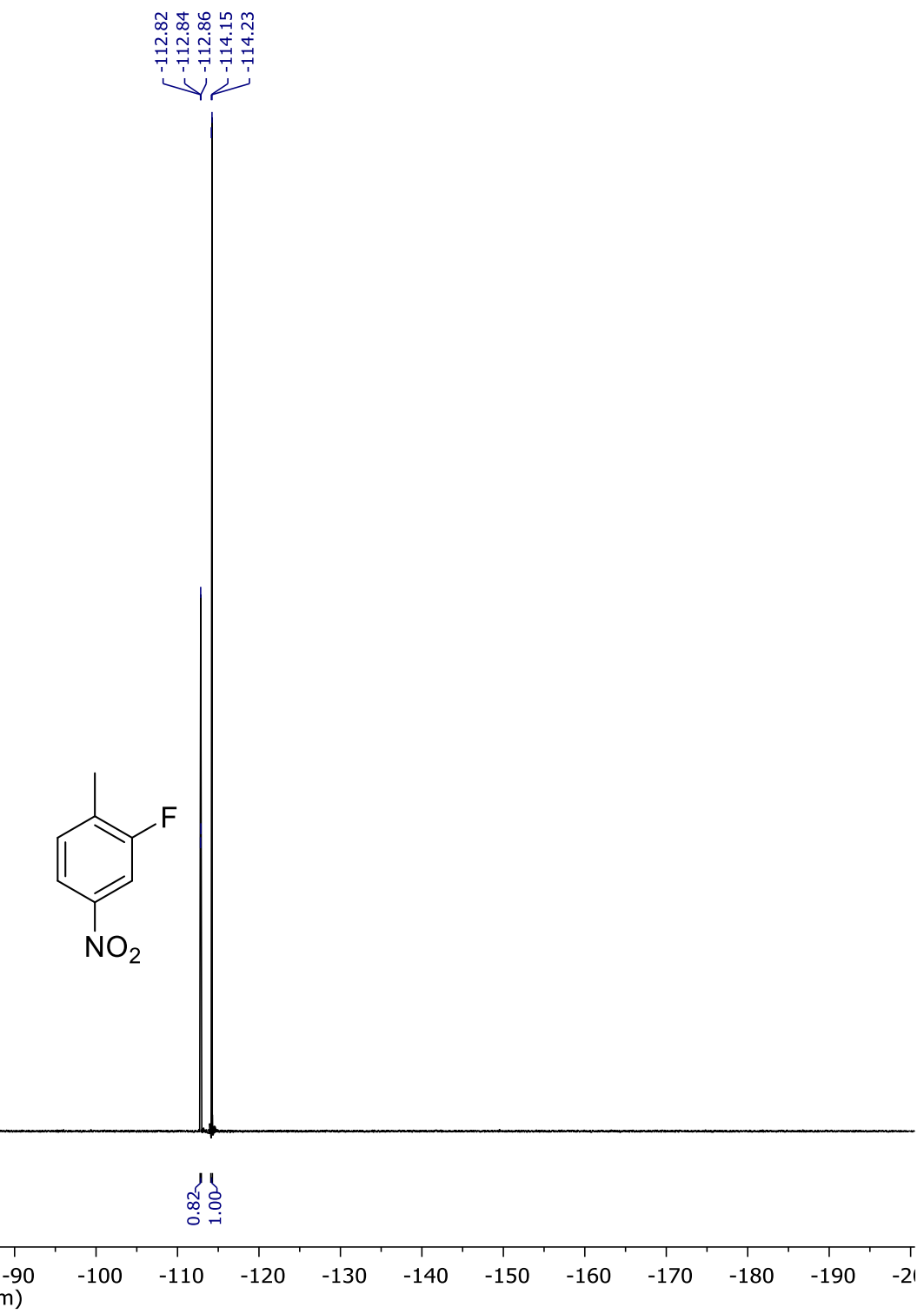




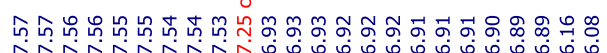
作

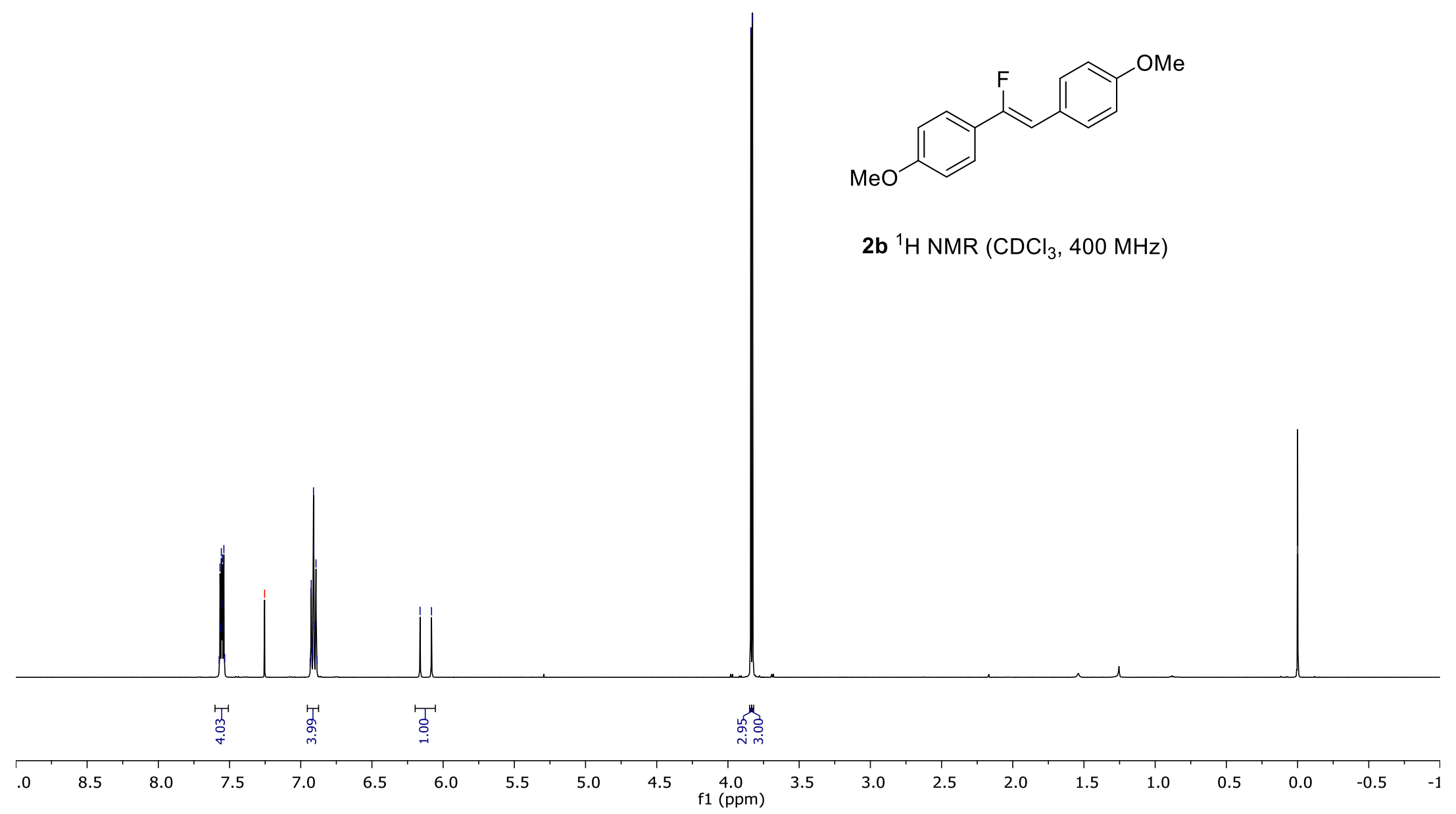




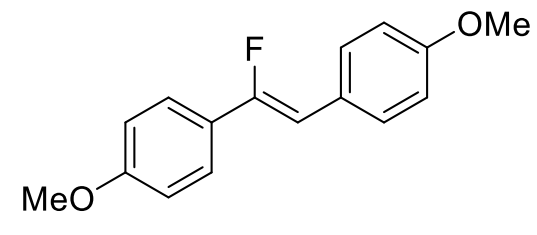

2b ${ }^{1} \mathrm{H} \mathrm{NMR}\left(\mathrm{CDCl}_{3}, 470 \mathrm{MHz}\right)$

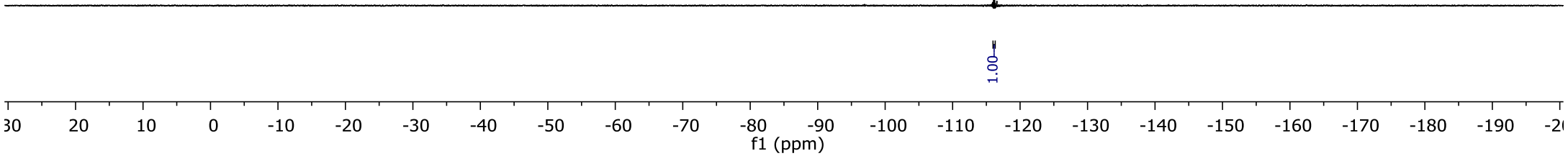




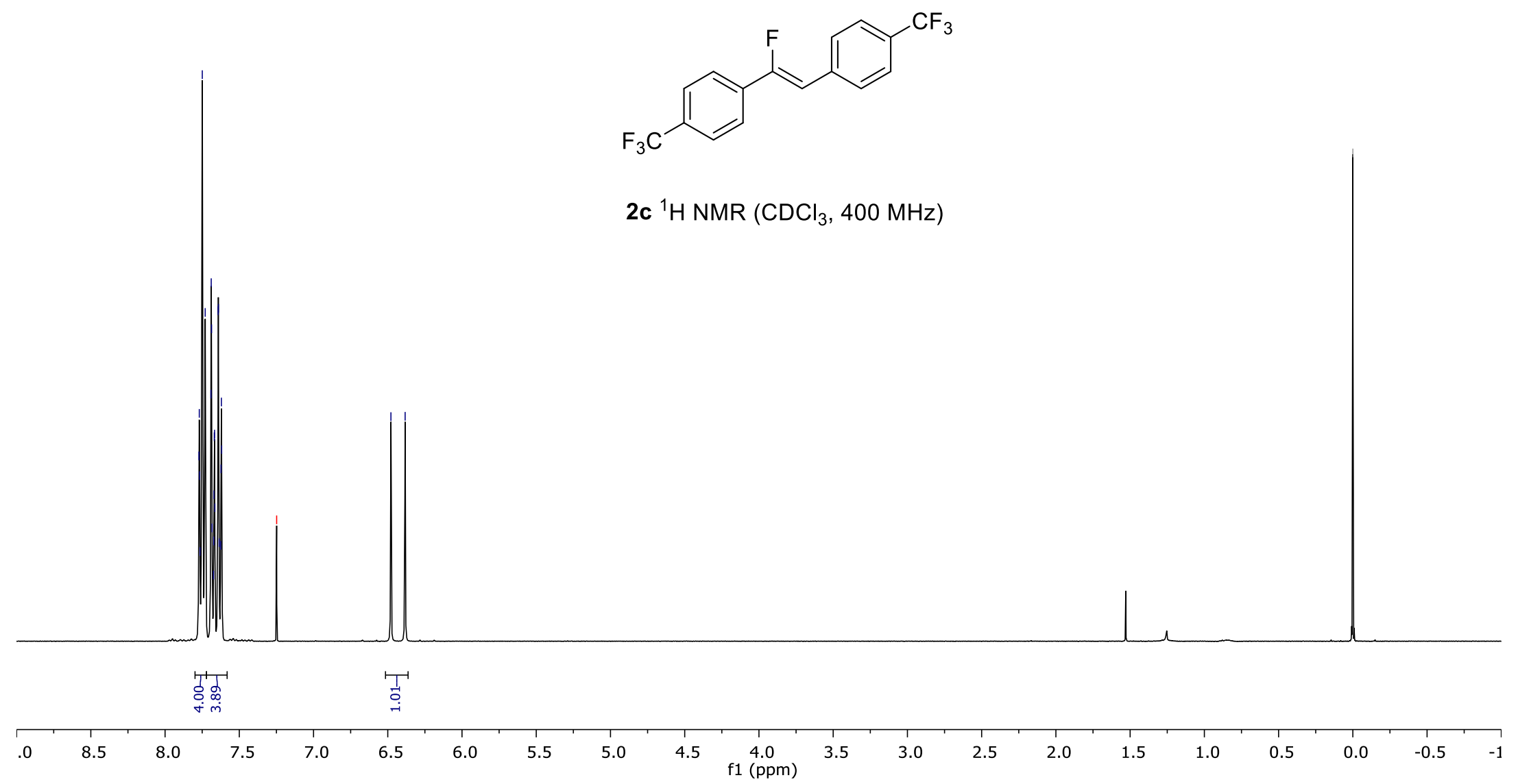



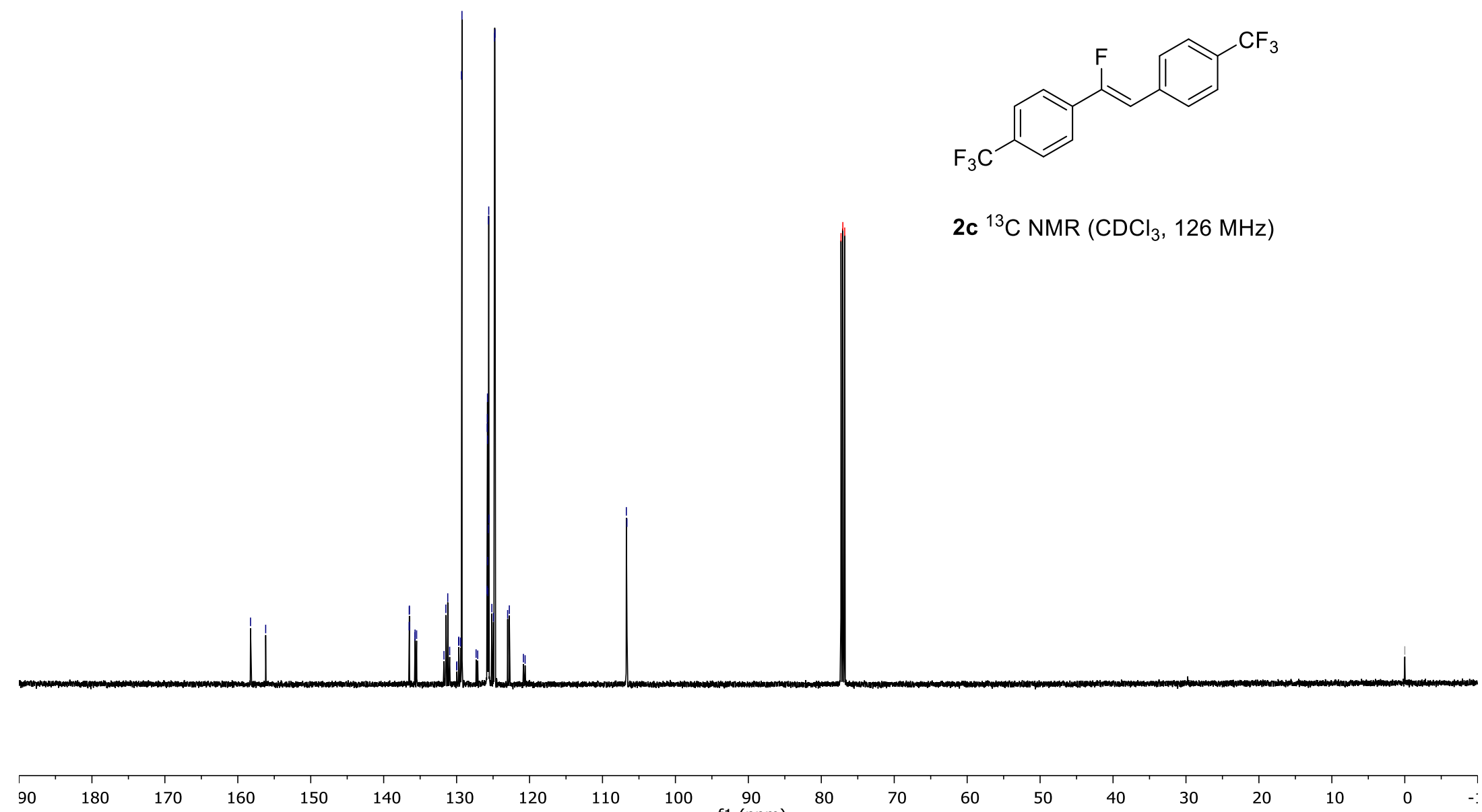

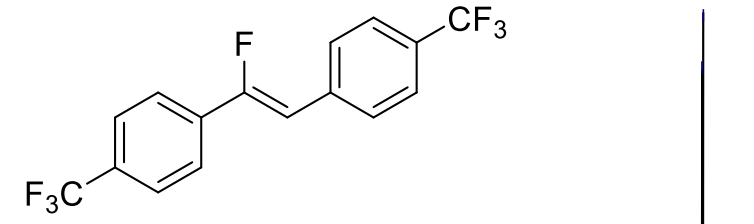

2c ${ }^{19} \mathrm{~F} \mathrm{NMR}\left(\mathrm{CDCl}_{3}, 376 \mathrm{MHz}\right)$

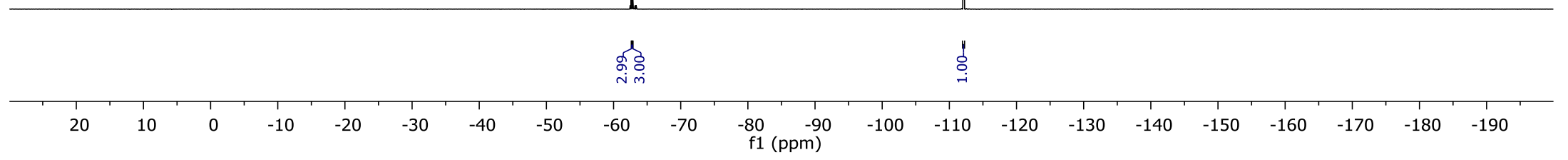




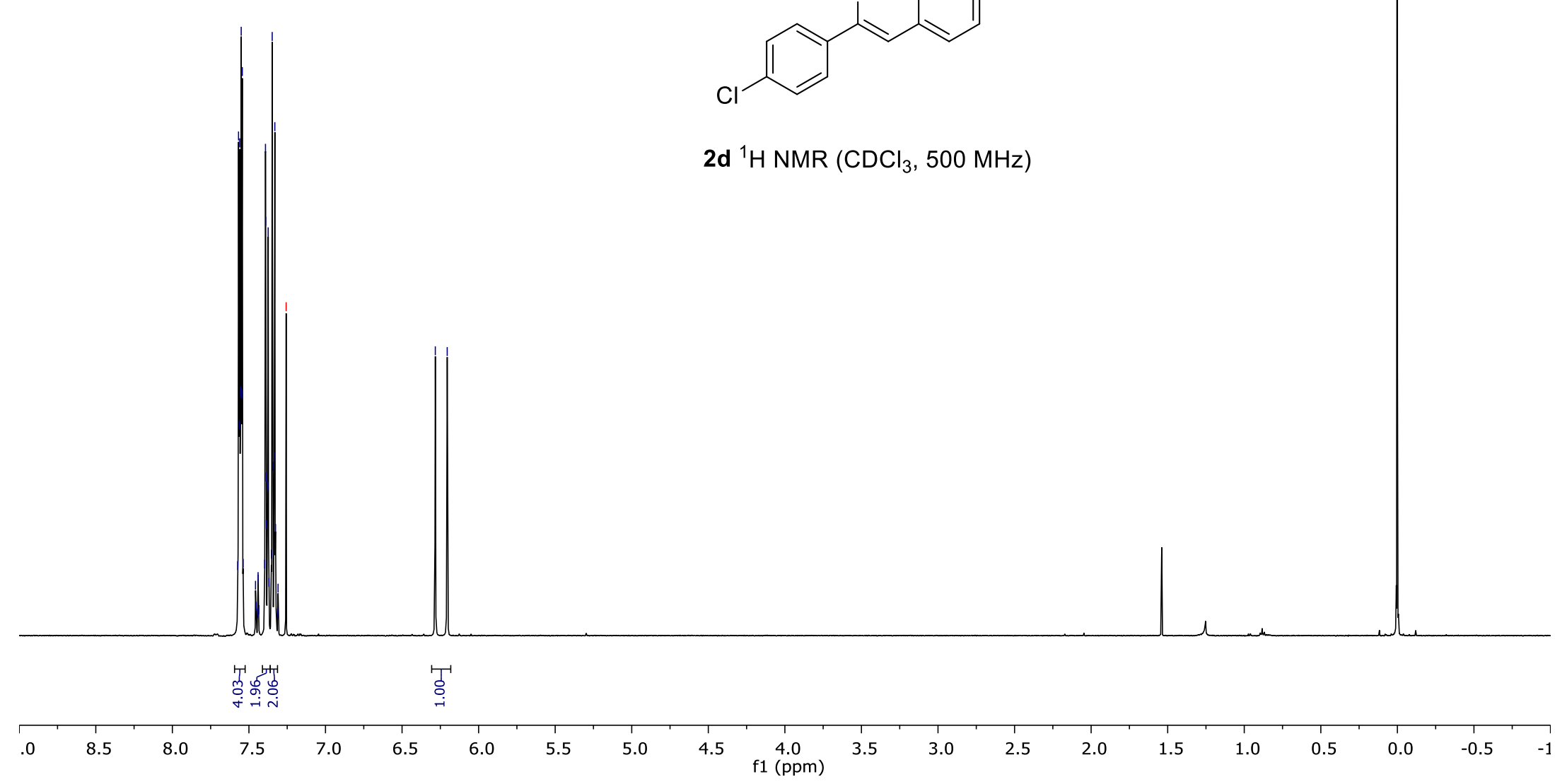




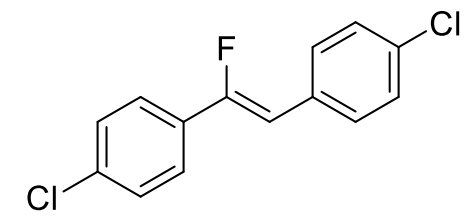

2d ${ }^{19} \mathrm{~F} \mathrm{NMR}\left(\mathrm{CDCl}_{3}, 470 \mathrm{MHz}\right)$

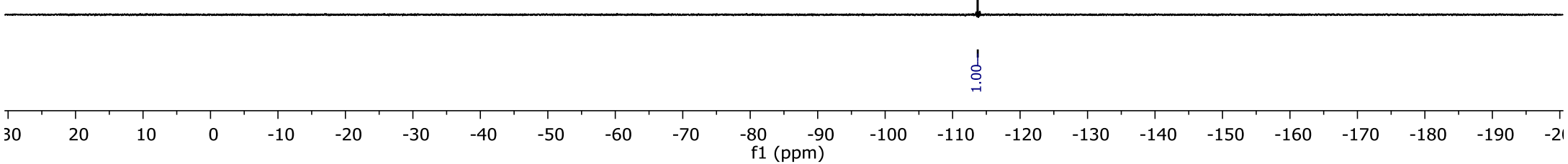




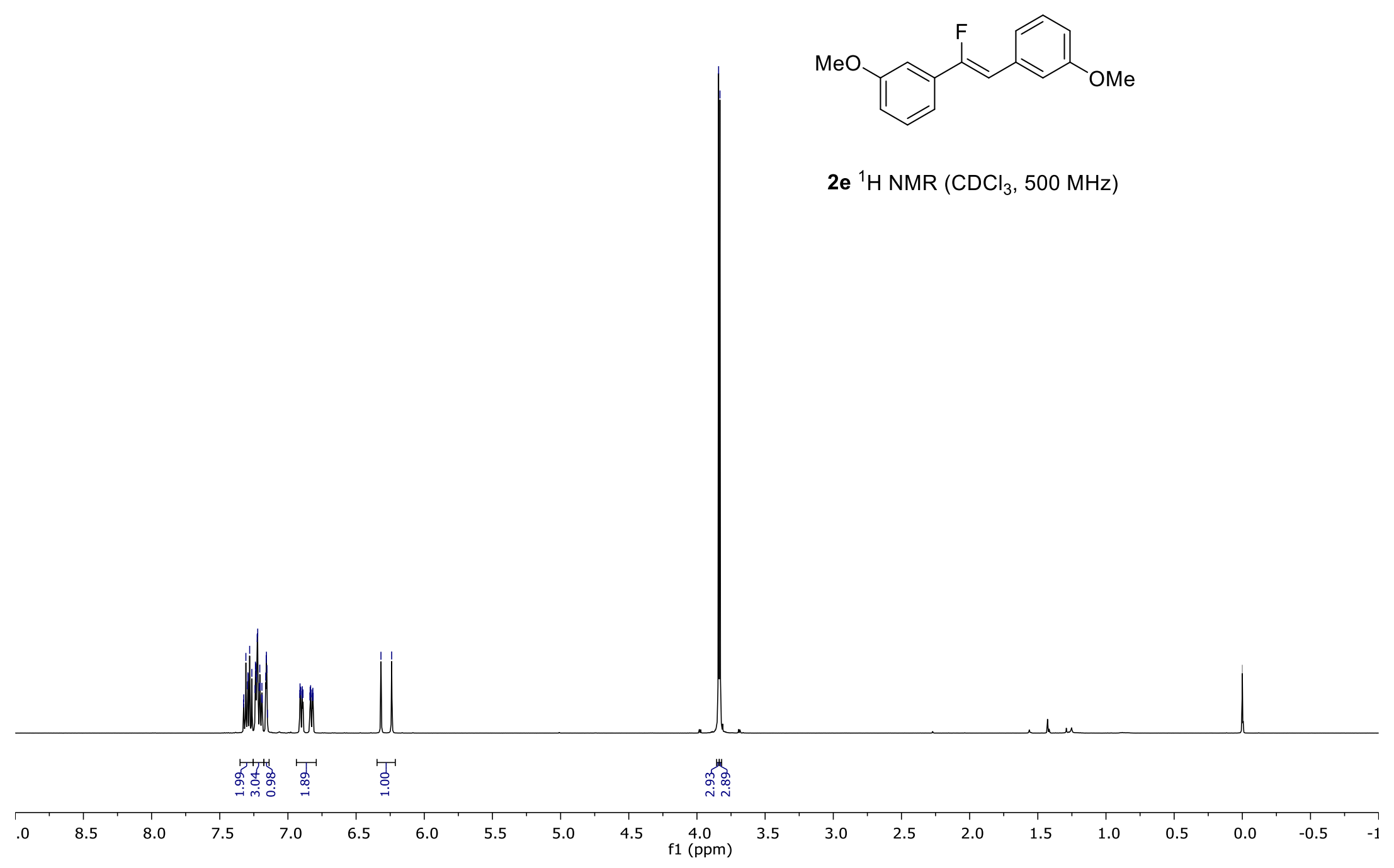




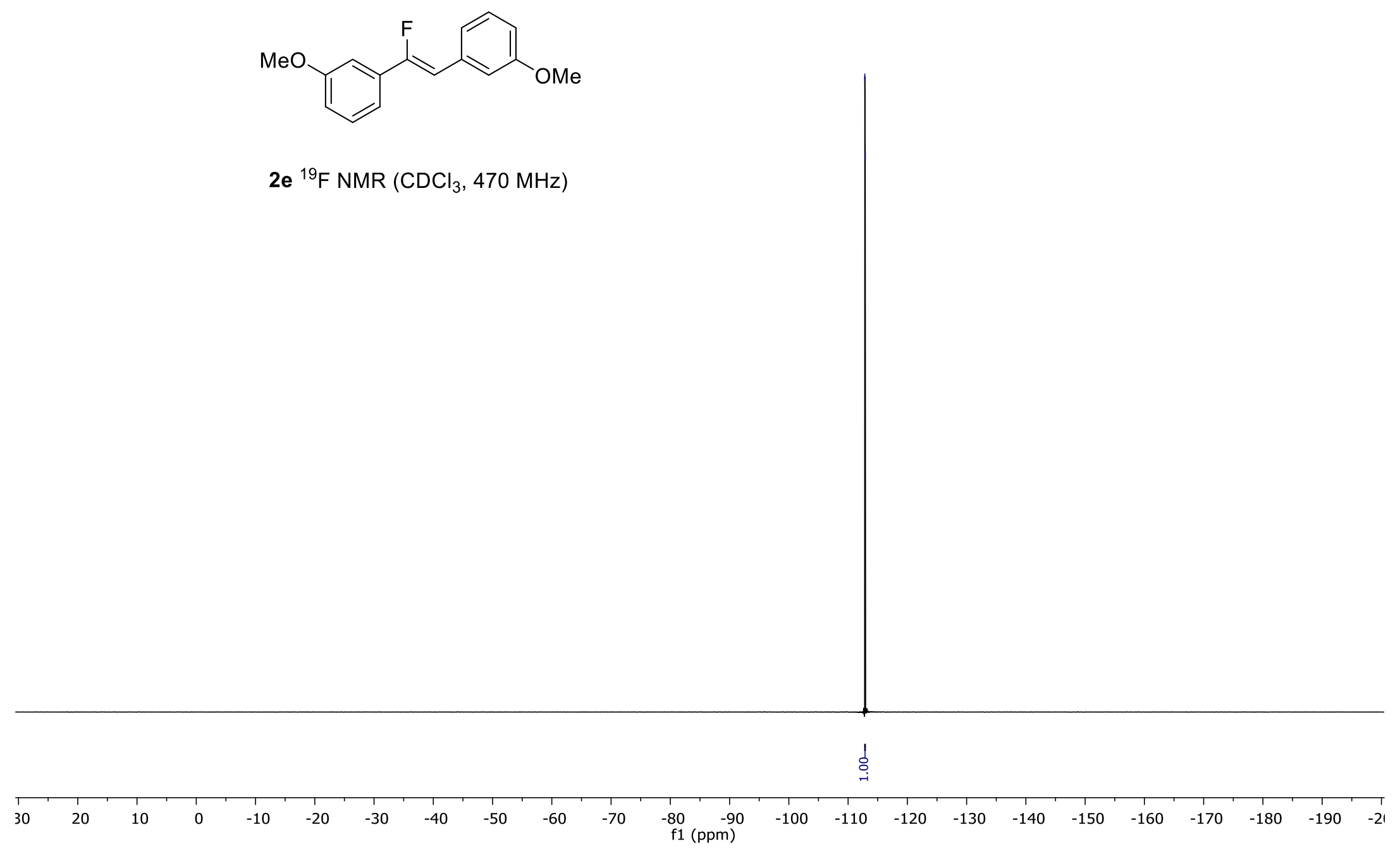

$2 e^{19} \mathrm{~F} \mathrm{NMR}\left(\mathrm{CDCl}_{3}, 470 \mathrm{MHz}\right)$ 


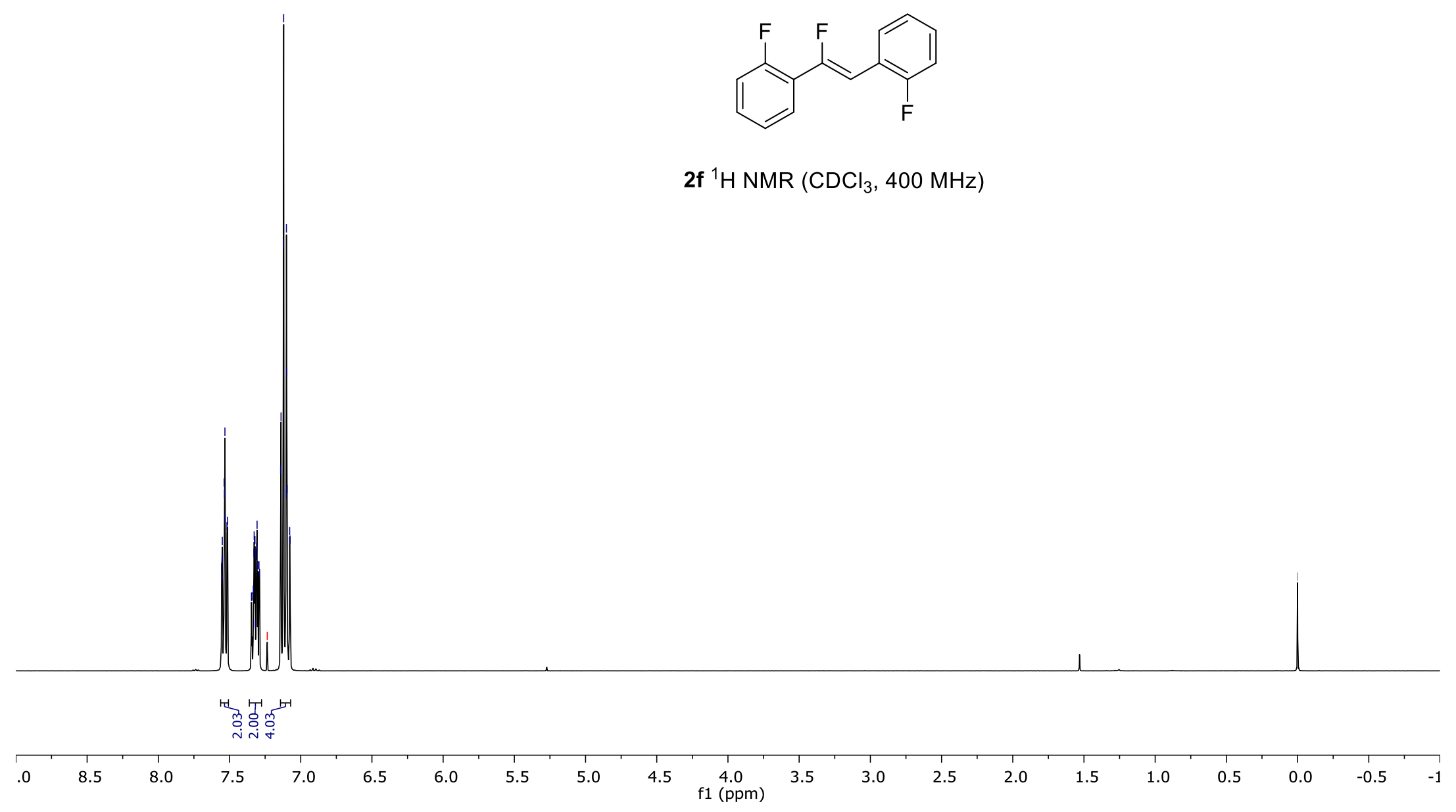



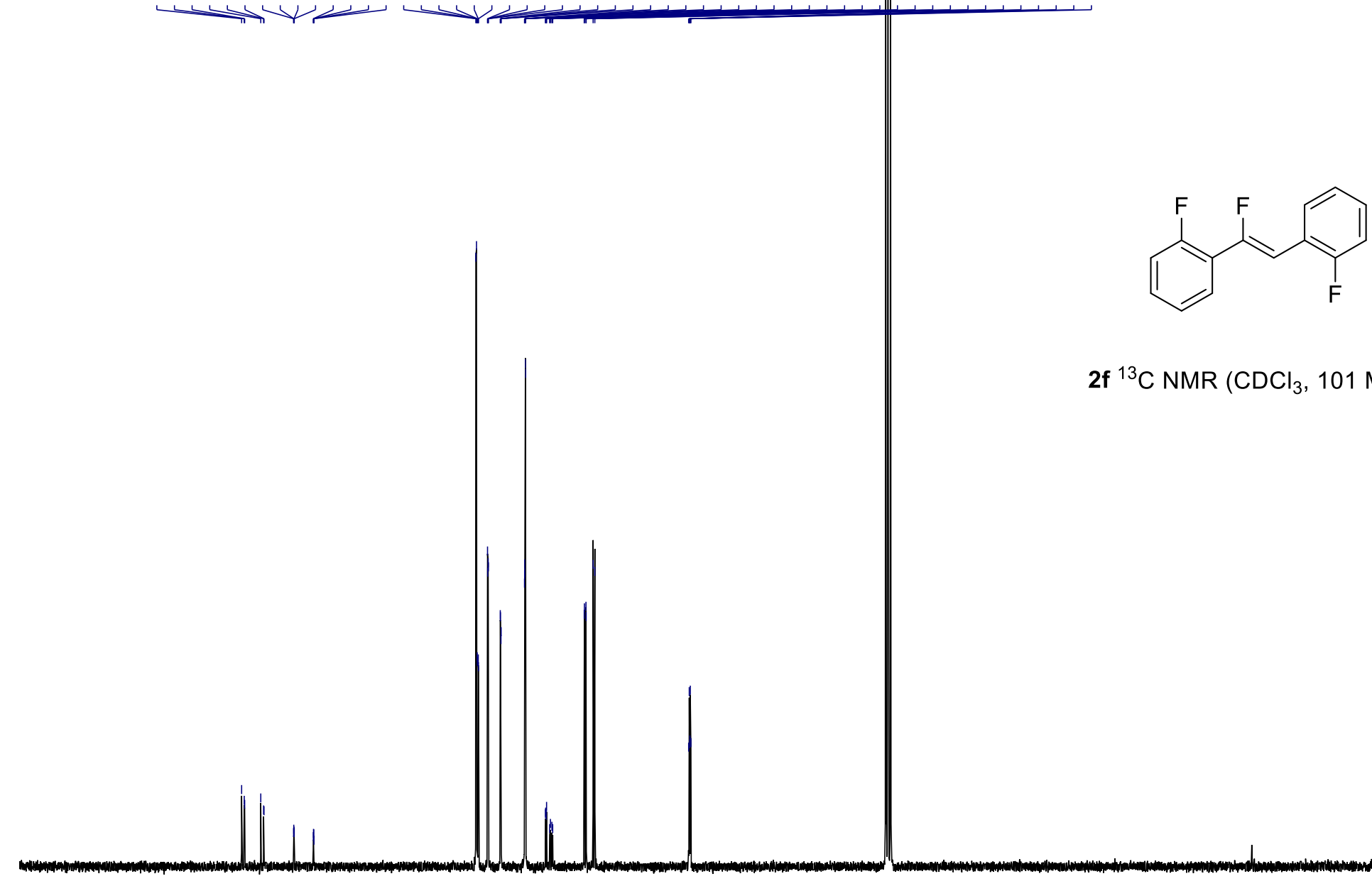

$2 \mathbf{f}^{13} \mathrm{C} \mathrm{NMR}\left(\mathrm{CDCl}_{3}, 101 \mathrm{MHz}\right)$
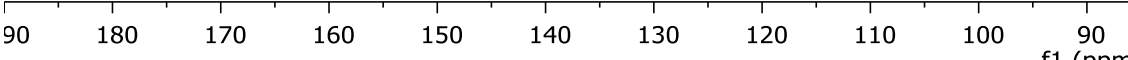

80

70

60

50

40

30 


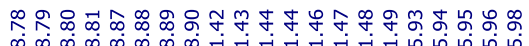

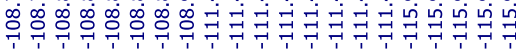

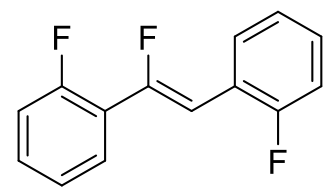

2f ${ }^{19} \mathrm{~F} \mathrm{NMR}\left(\mathrm{CDCl}_{3}, 470 \mathrm{MHz}\right)$

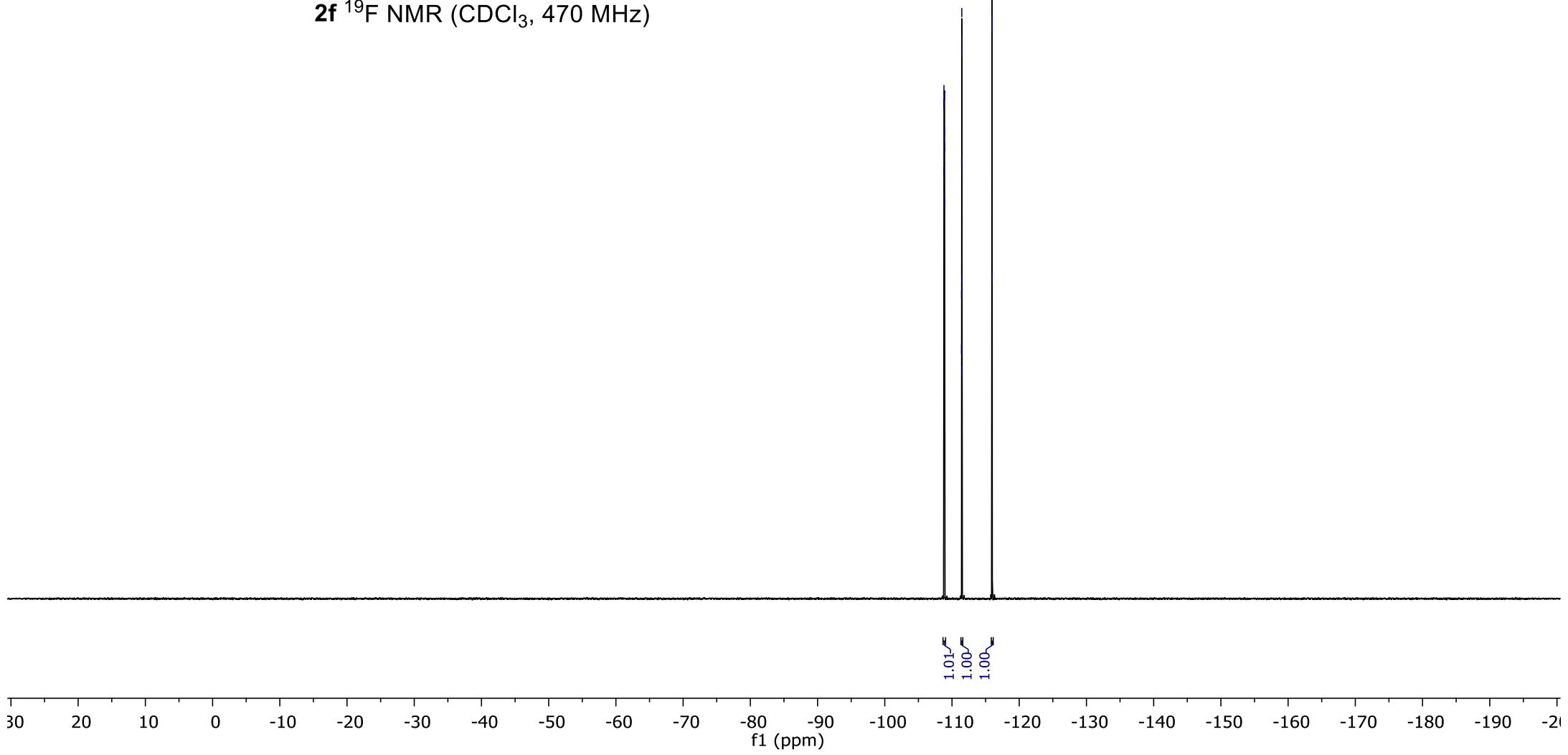




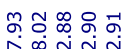

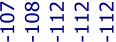

$\checkmark \psi$

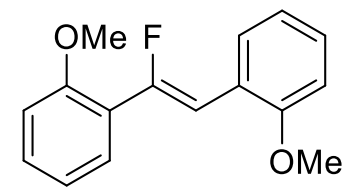

$2 \mathbf{g}^{19} \mathrm{~F} \mathrm{NMR}\left(\mathrm{CDCl}_{3}, 470 \mathrm{MHz}\right)$
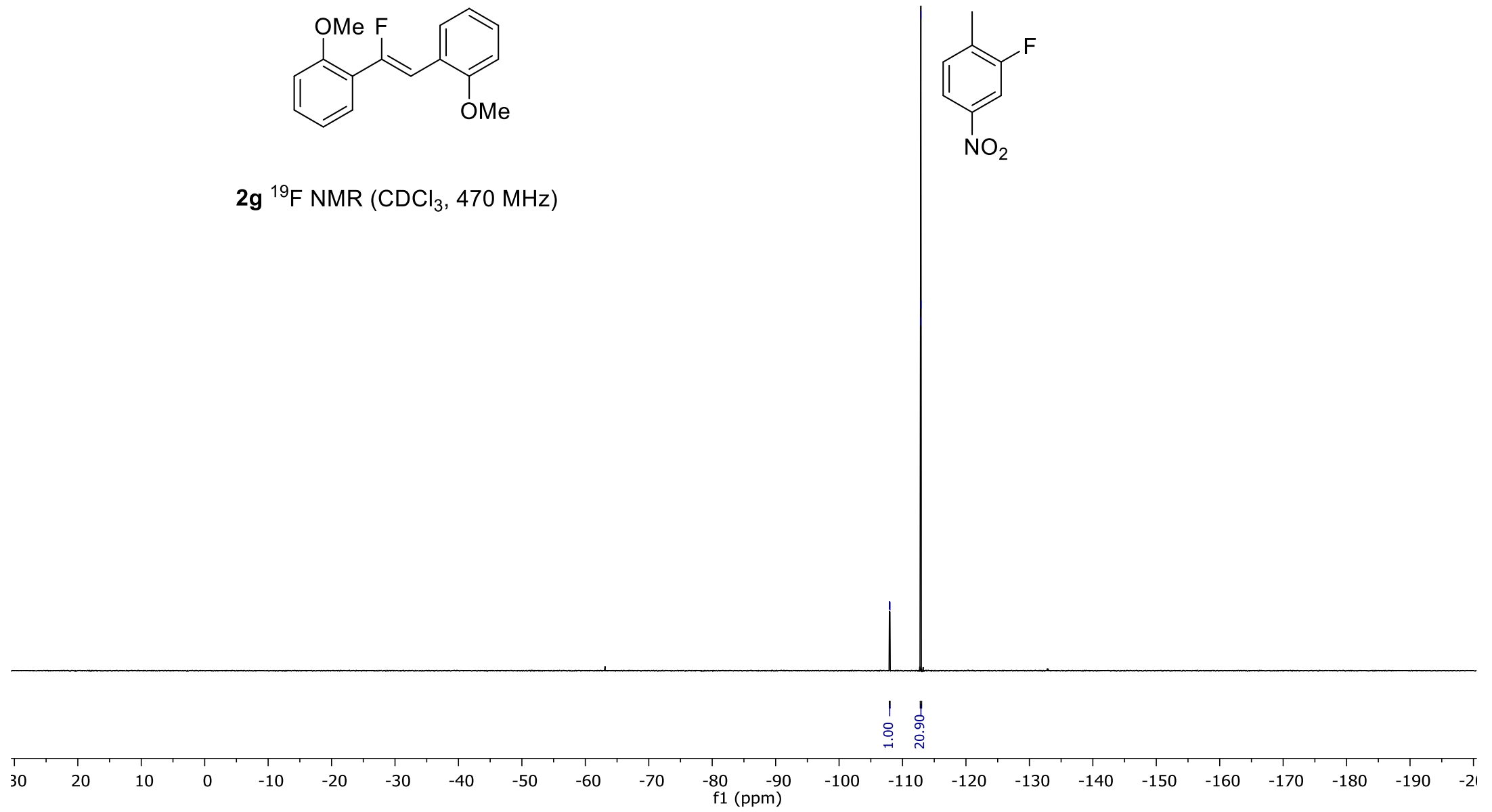


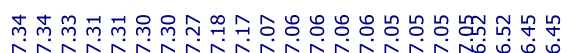

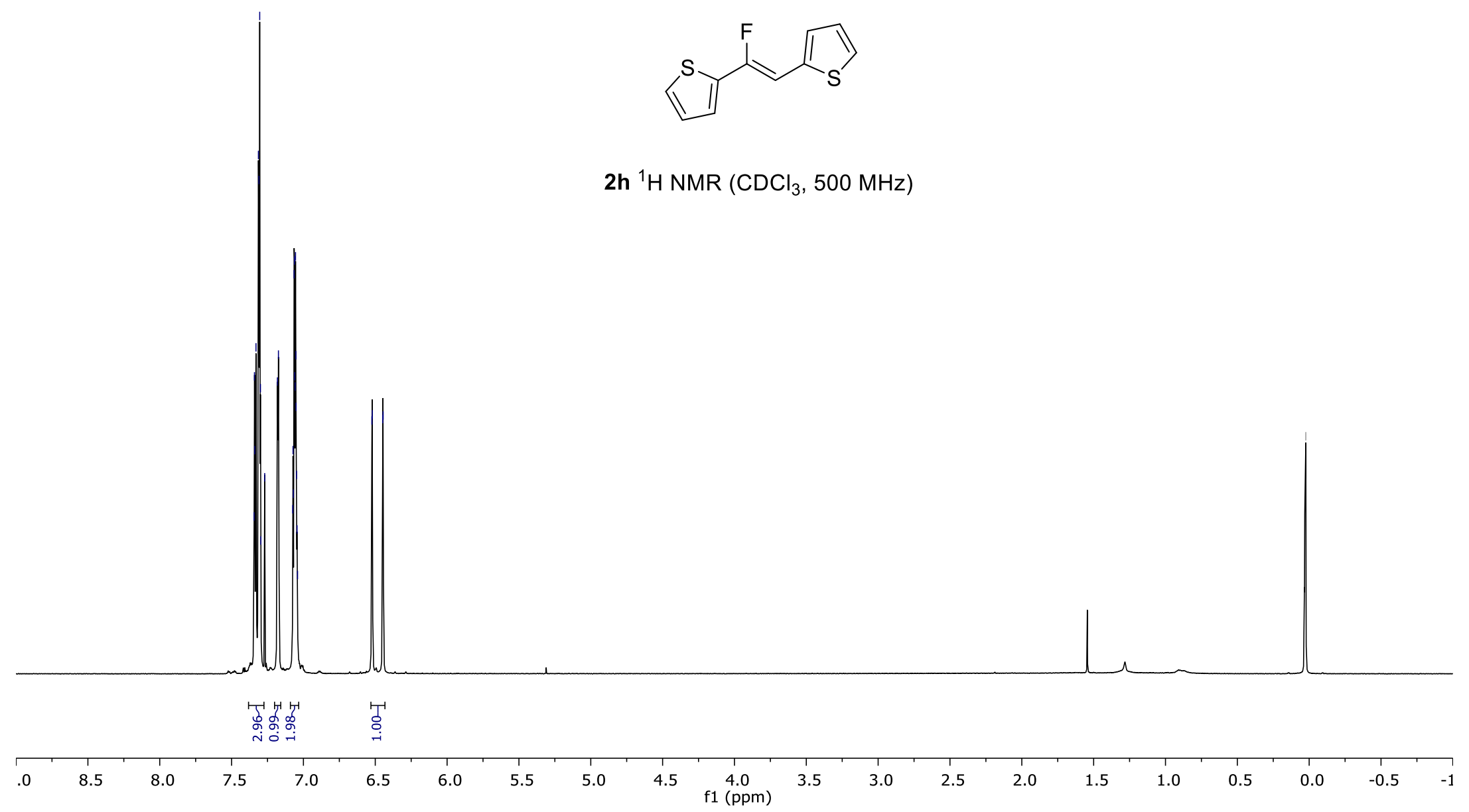




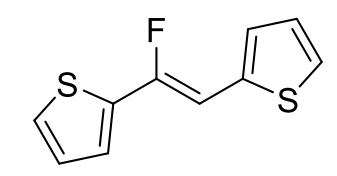

2h ${ }^{19} \mathrm{~F} \mathrm{NMR}\left(\mathrm{CDCl}_{3}, 470 \mathrm{MHz}\right)$

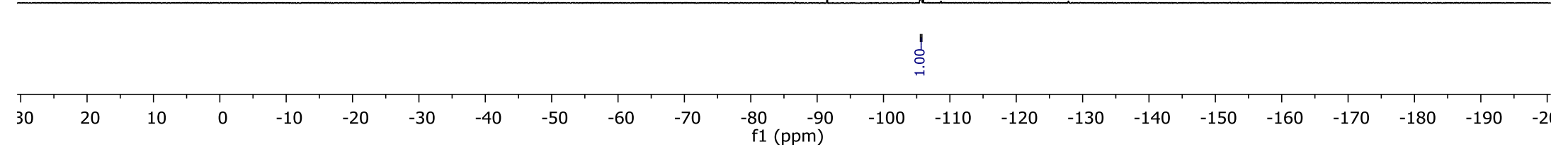




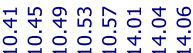

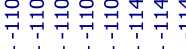

w

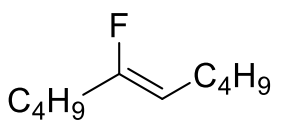

$2 \mathbf{i}^{19} \mathrm{~F} \mathrm{NMR}\left(\mathrm{CDCl}_{3}, 470 \mathrm{MHz}\right)$
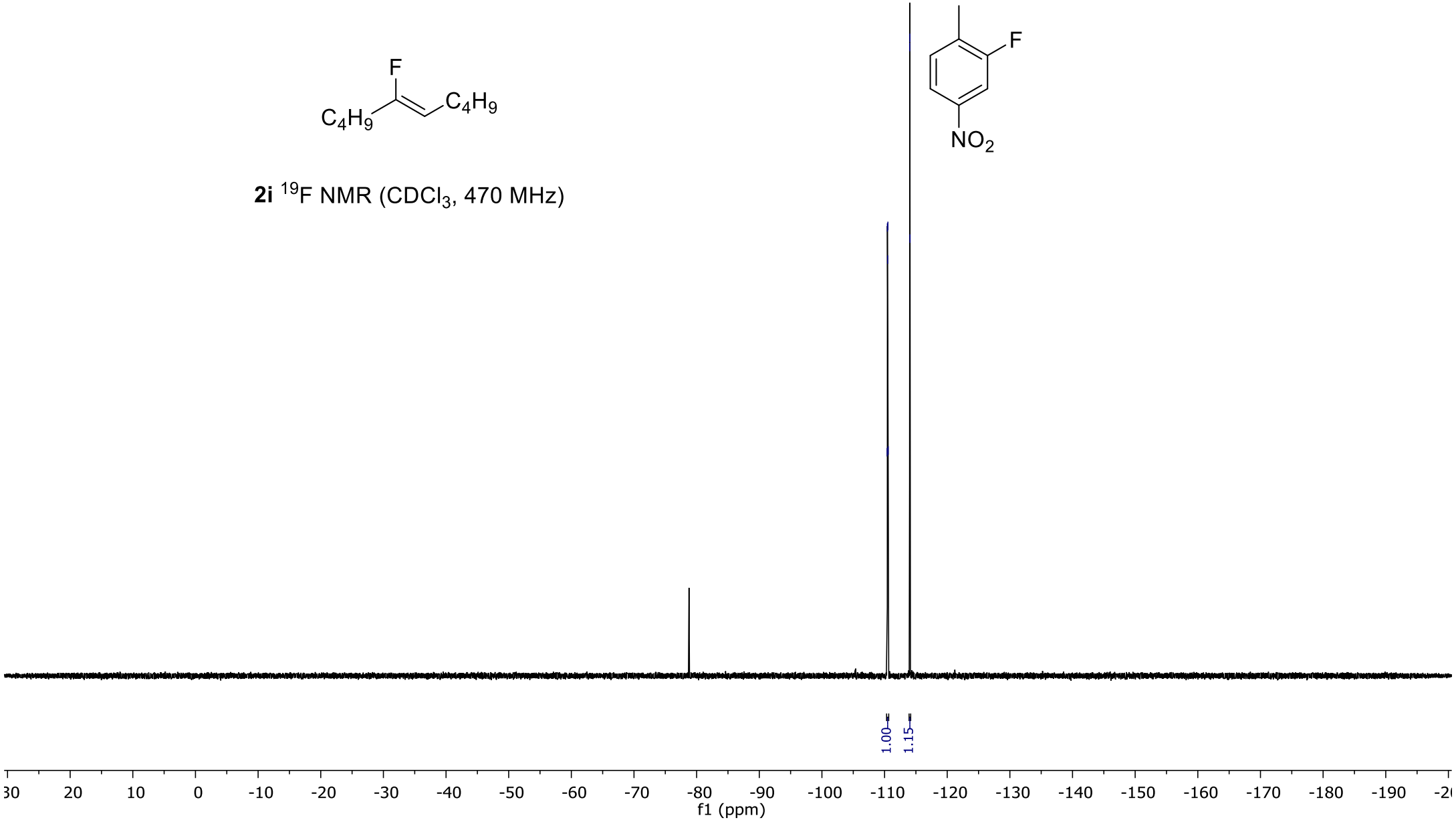


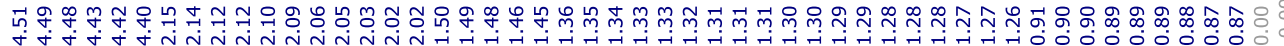

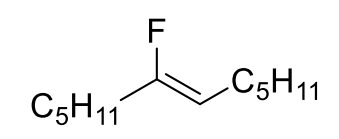

2j ${ }^{1} \mathrm{H}$ NMR $\left(\mathrm{CDCl}_{3}, 500 \mathrm{MHz}\right)$

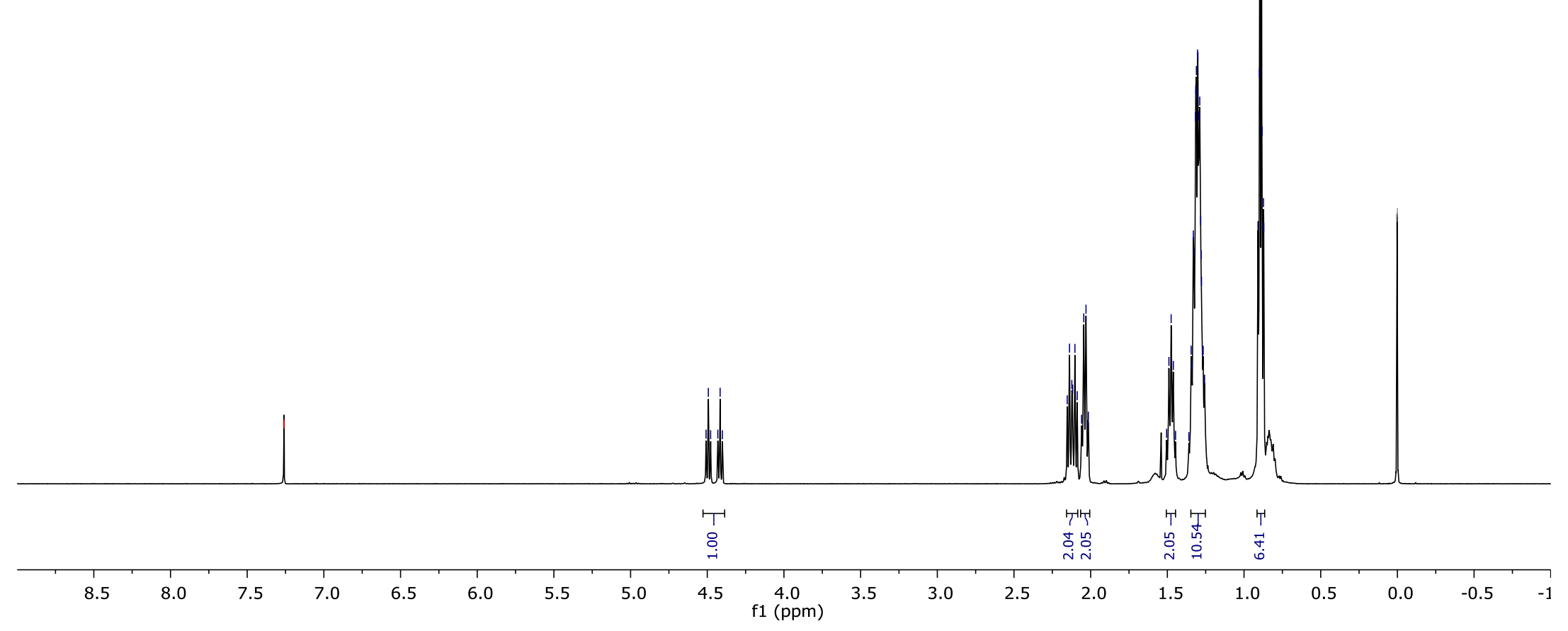




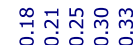

윅후국욱

iv

$\mathrm{C}_{5} \mathrm{H}_{11} \stackrel{\mathrm{F}}{\mathrm{L}} \mathrm{C}_{5} \mathrm{H}_{11}$

2j ${ }^{19} \mathrm{~F} \mathrm{NMR}\left(\mathrm{CDCl}_{3}, 470 \mathrm{MHz}\right)$

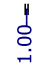

\begin{tabular}{llllllllllllllllllllllll}
\hline 30 & 20 & 10 & 0 & -10 & -20 & -30 & -40 & -50 & -60 & -70 & -80 & -90 & -100 & -110 & -120 & -130 & -140 & -150 & -160 & -170 & -180 & -190 & -2 \\
& & & & & & &
\end{tabular}




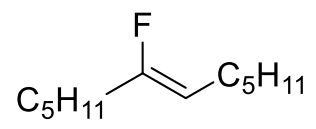

2j ${ }^{13} \mathrm{C} \mathrm{NMR}\left(\mathrm{CDCl}_{3}, 101 \mathrm{MHz}\right)$
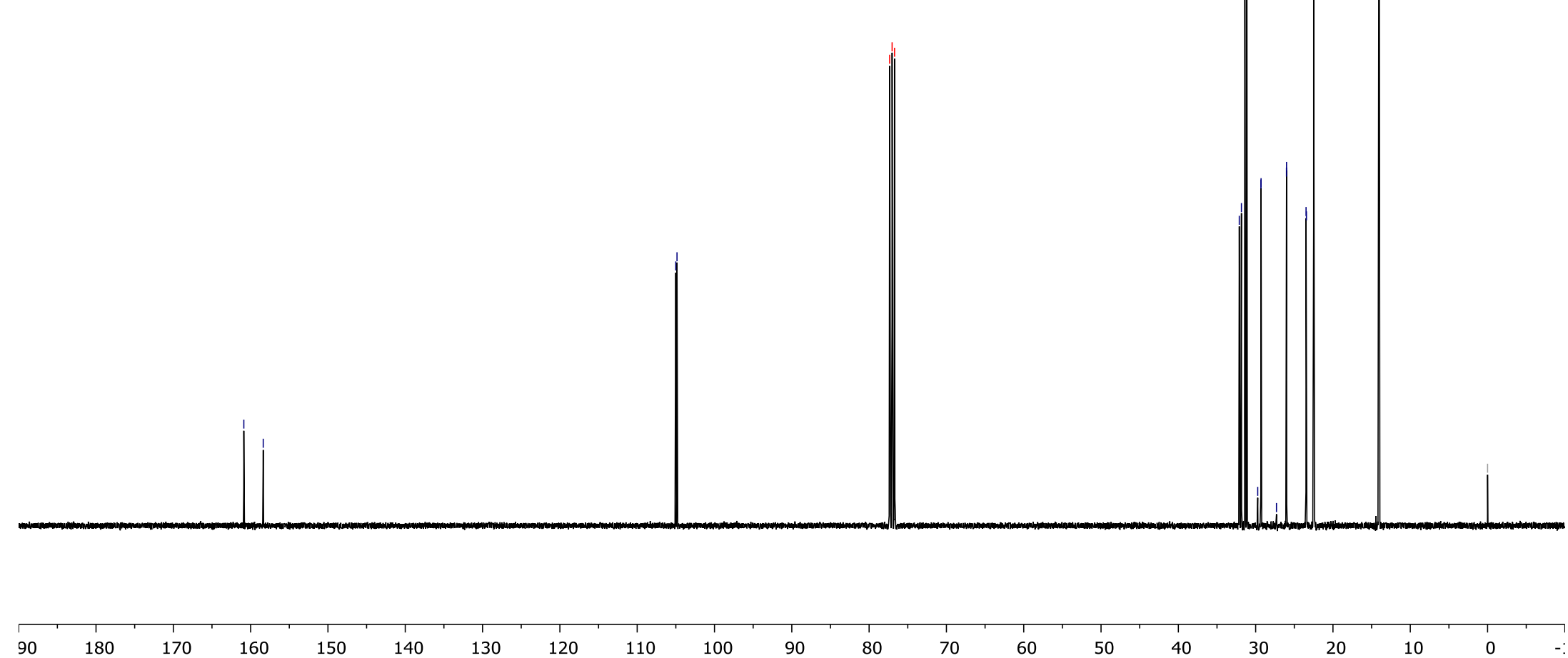

100 


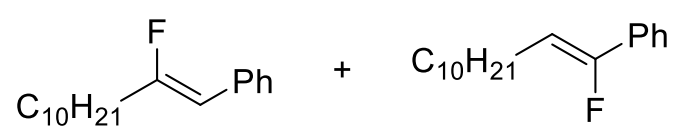

$2 \mathbf{k}+2 \mathbf{k}^{\prime}(69: \mathbf{3 1}){ }^{1} \mathrm{H}$ NMR $\left(\mathrm{CDCl}_{3}, 500 \mathrm{MHz}\right)$

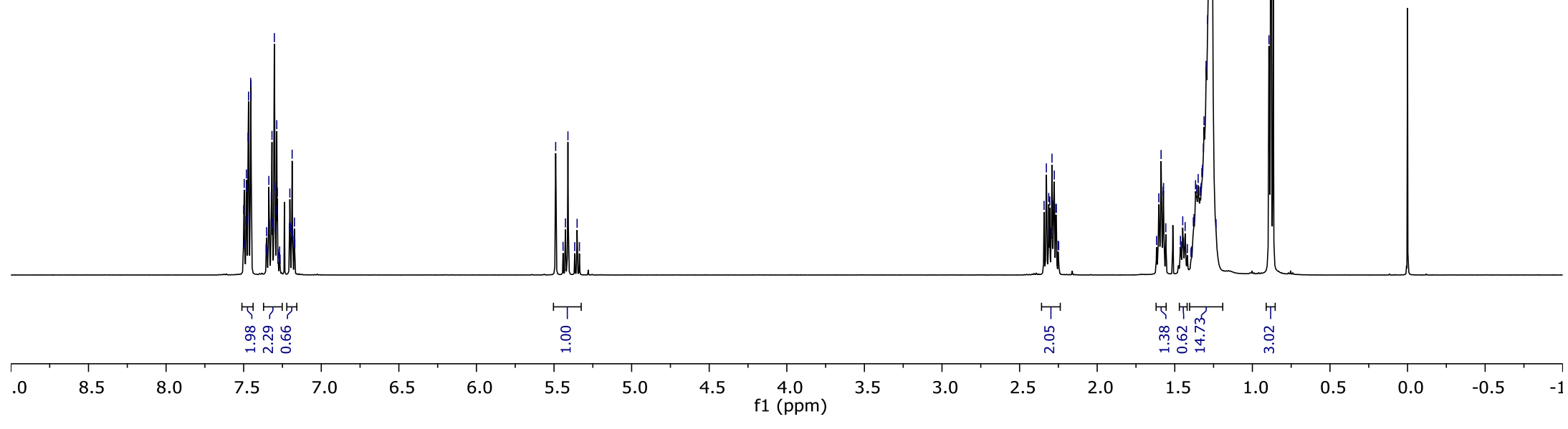




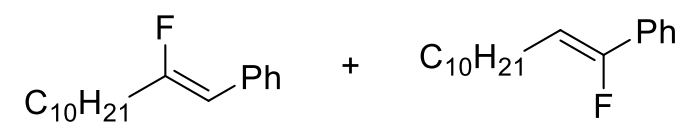

$2 \mathbf{k}+2 \mathbf{k}^{\prime}(69: \mathbf{3 1}){ }^{13} \mathrm{C} \mathrm{NMR}\left(\mathrm{CDCl}_{3}, 126 \mathrm{MHz}\right)$

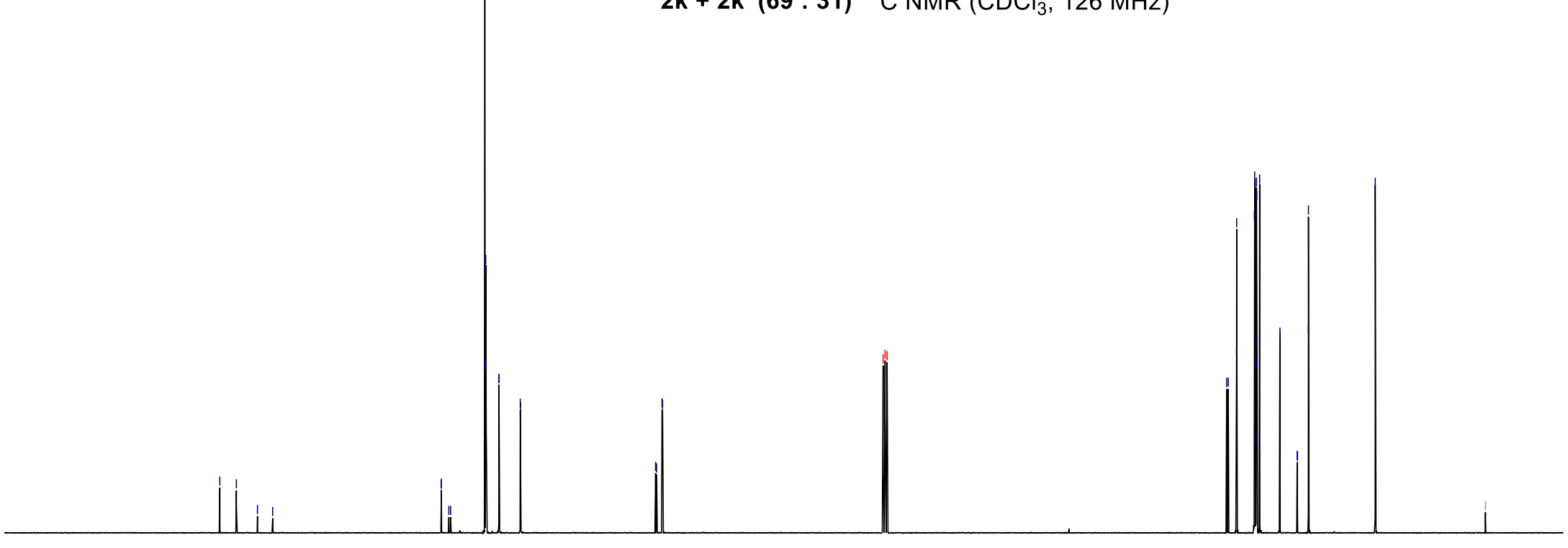




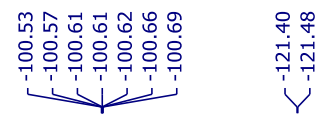

$\mathrm{C}_{10} \mathrm{H}_{21} \stackrel{\mathrm{F}}{\mathrm{Ph}}+\mathrm{C}_{10} \mathrm{H}_{21} \overbrace{\mathrm{F}}^{\mathrm{Ph}}$

2k + 2k' (69: 31) ${ }^{19} \mathrm{~F} \mathrm{NMR}\left(\mathrm{CDCl}_{3}, 470 \mathrm{MHz}\right)$

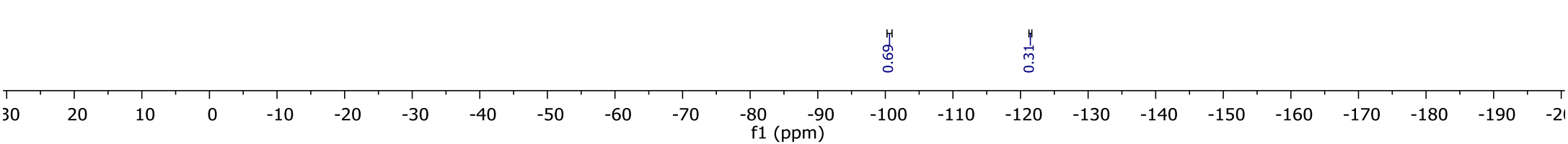




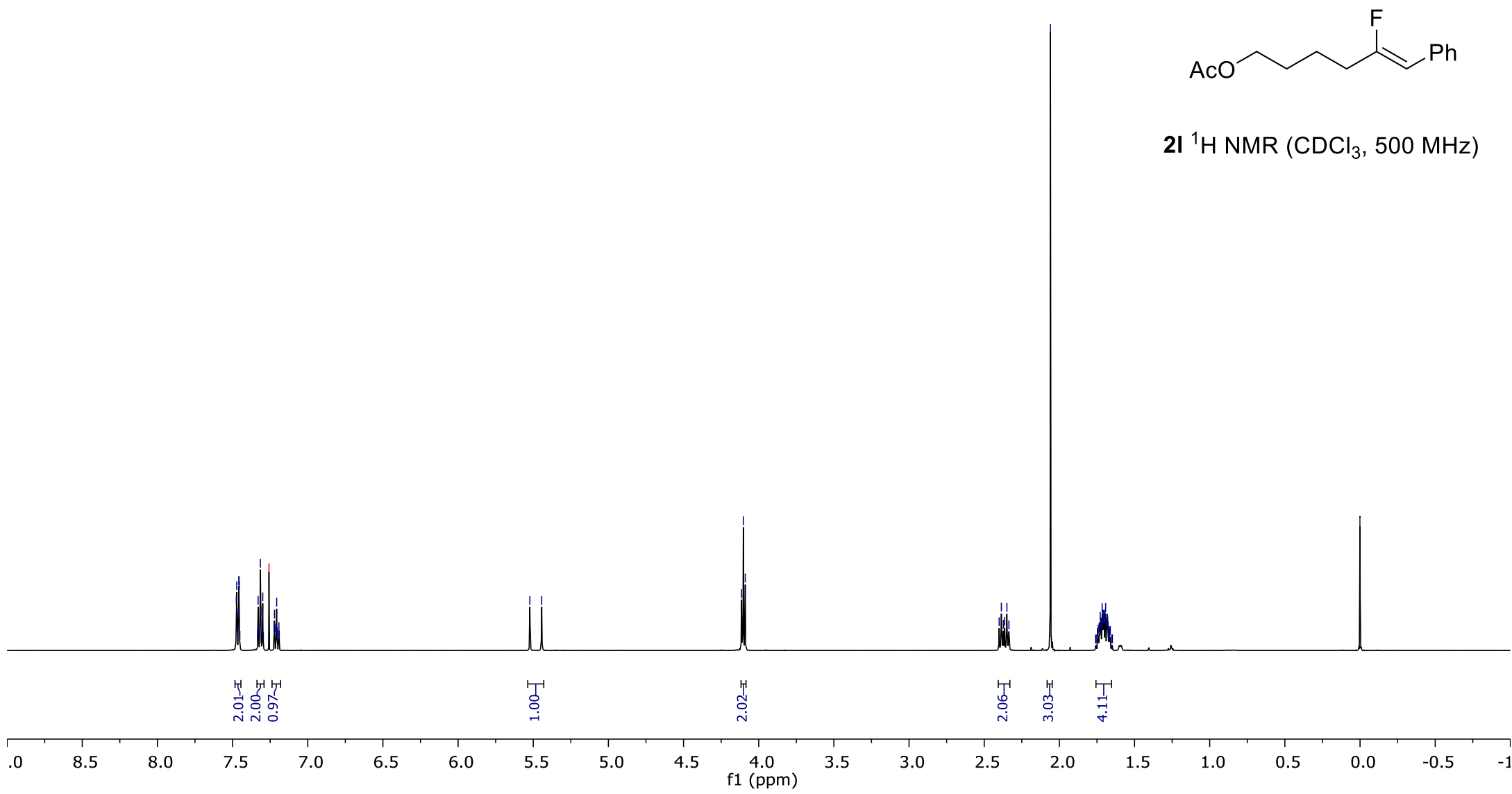

2I ${ }^{1} \mathrm{H}$ NMR $\left(\mathrm{CDCl}_{3}, 500 \mathrm{MHz}\right)$ 


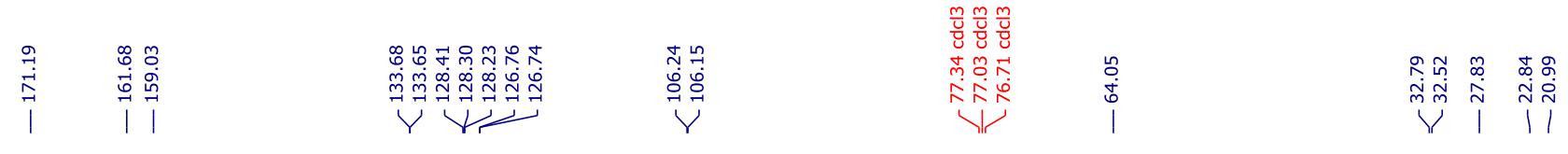

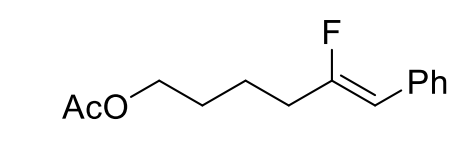

2I ${ }^{13} \mathrm{C} \mathrm{NMR}\left(\mathrm{CDCl}_{3}, 101 \mathrm{MHz}\right)$

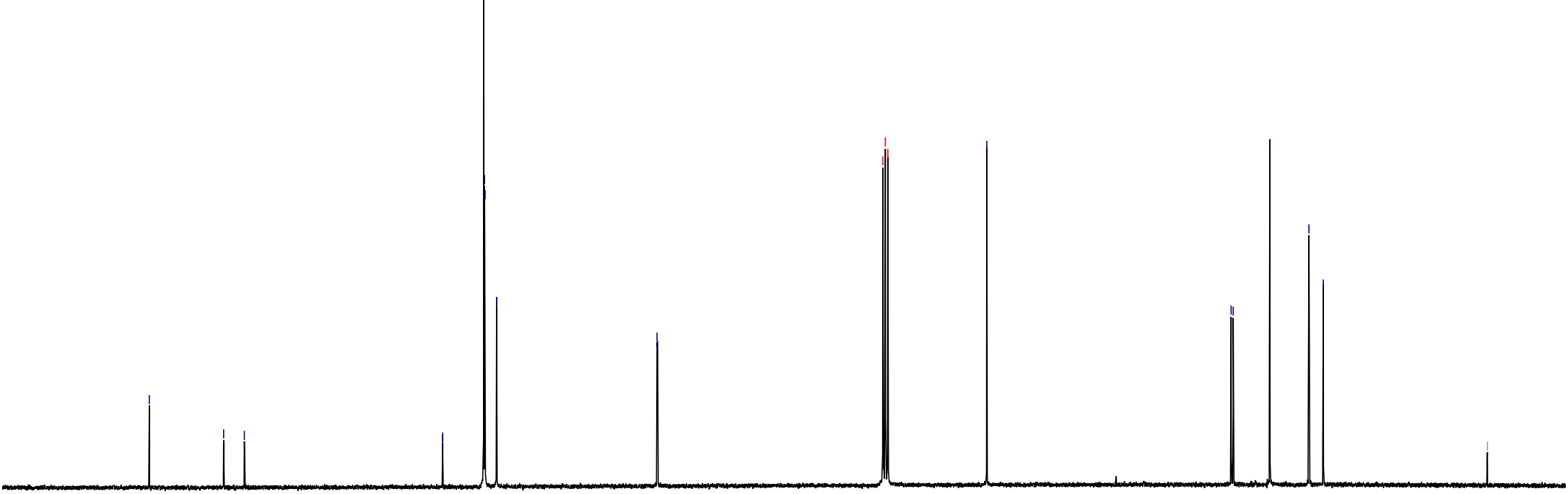

$90 \quad 180$

150

140

130

$120 \quad 110$

100

$\underset{f 1}{90} \quad 80 \quad 70 \quad 60$

50

40

30 
ㅇำ ㄸำ

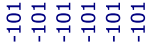

Y

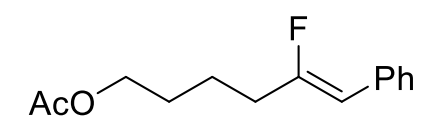

2I ${ }^{19} \mathrm{~F} \mathrm{NMR}\left(\mathrm{CDCl}_{3}, 470 \mathrm{MHz}\right)$

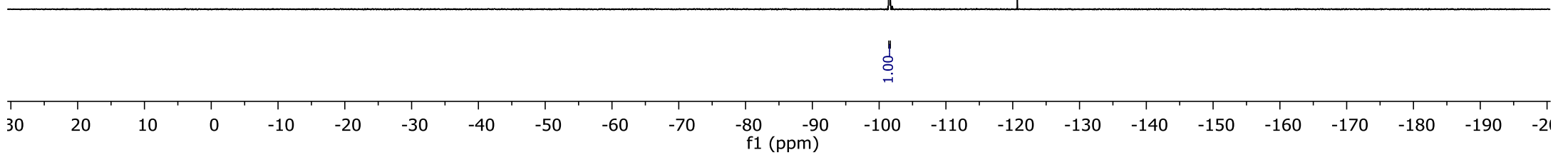




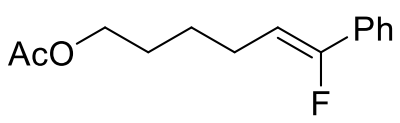

2I' ${ }^{1} \mathrm{H} \mathrm{NMR}\left(\mathrm{CDCl}_{3}, 500 \mathrm{MHz}\right)$

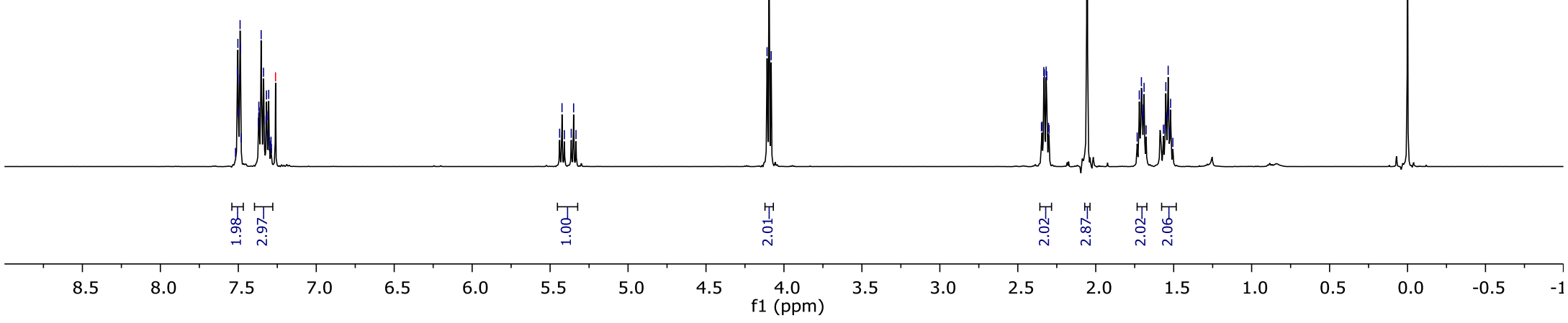




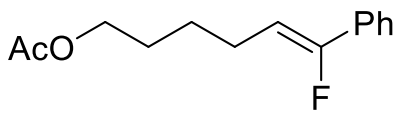

2I' ${ }^{13} \mathrm{C}$ NMR $\left(\mathrm{CDCl}_{3}, 101 \mathrm{MHz}\right)$
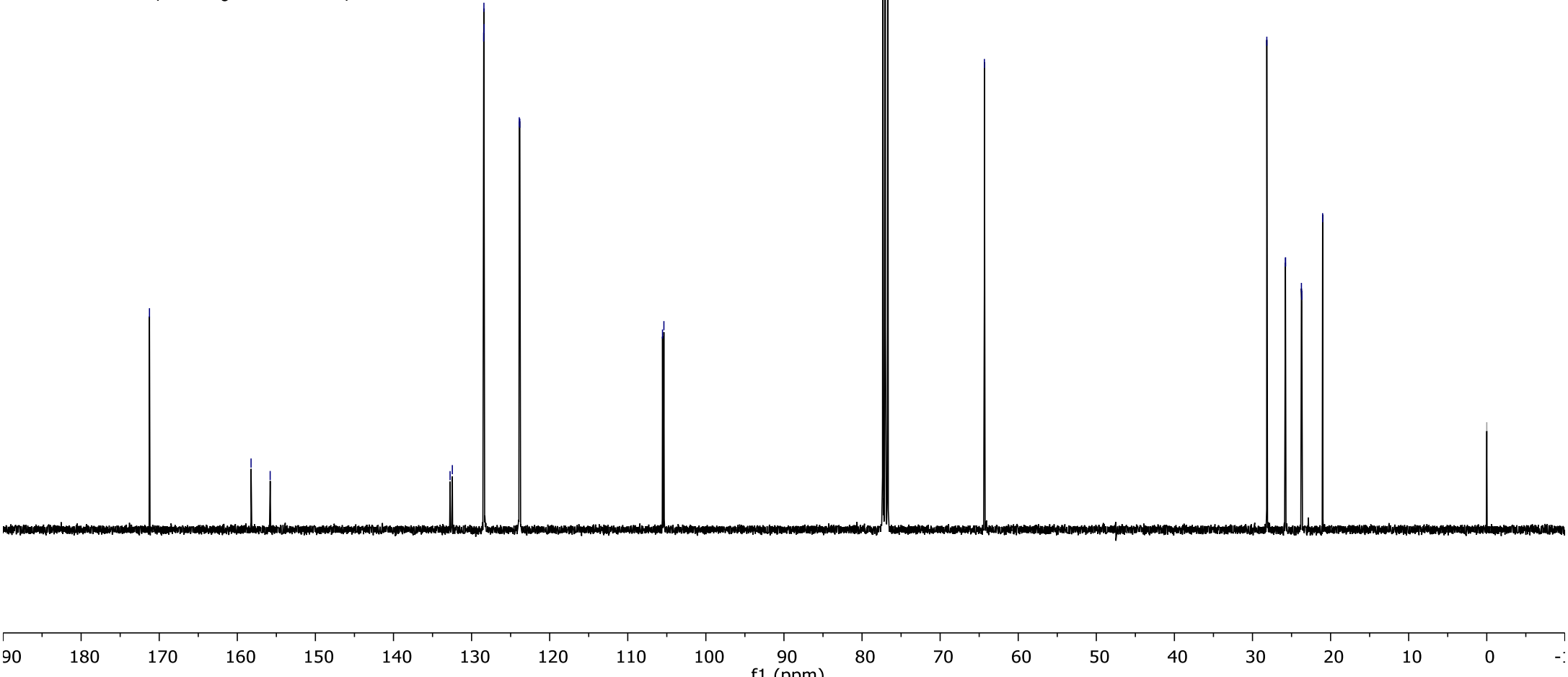


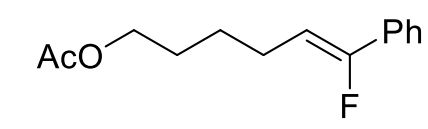

2l' ${ }^{19} \mathrm{~F} \mathrm{NMR}\left(\mathrm{CDCl}_{3}, 470 \mathrm{MHz}\right)$

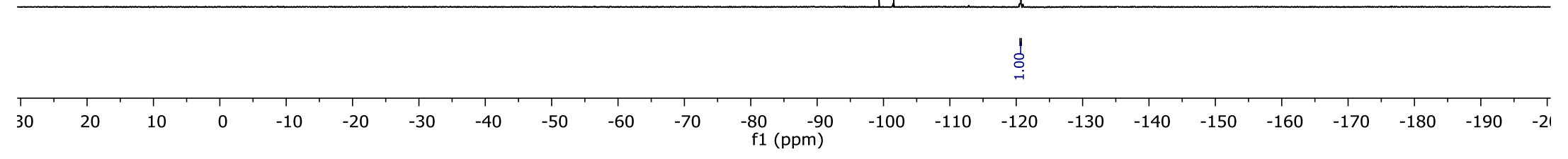




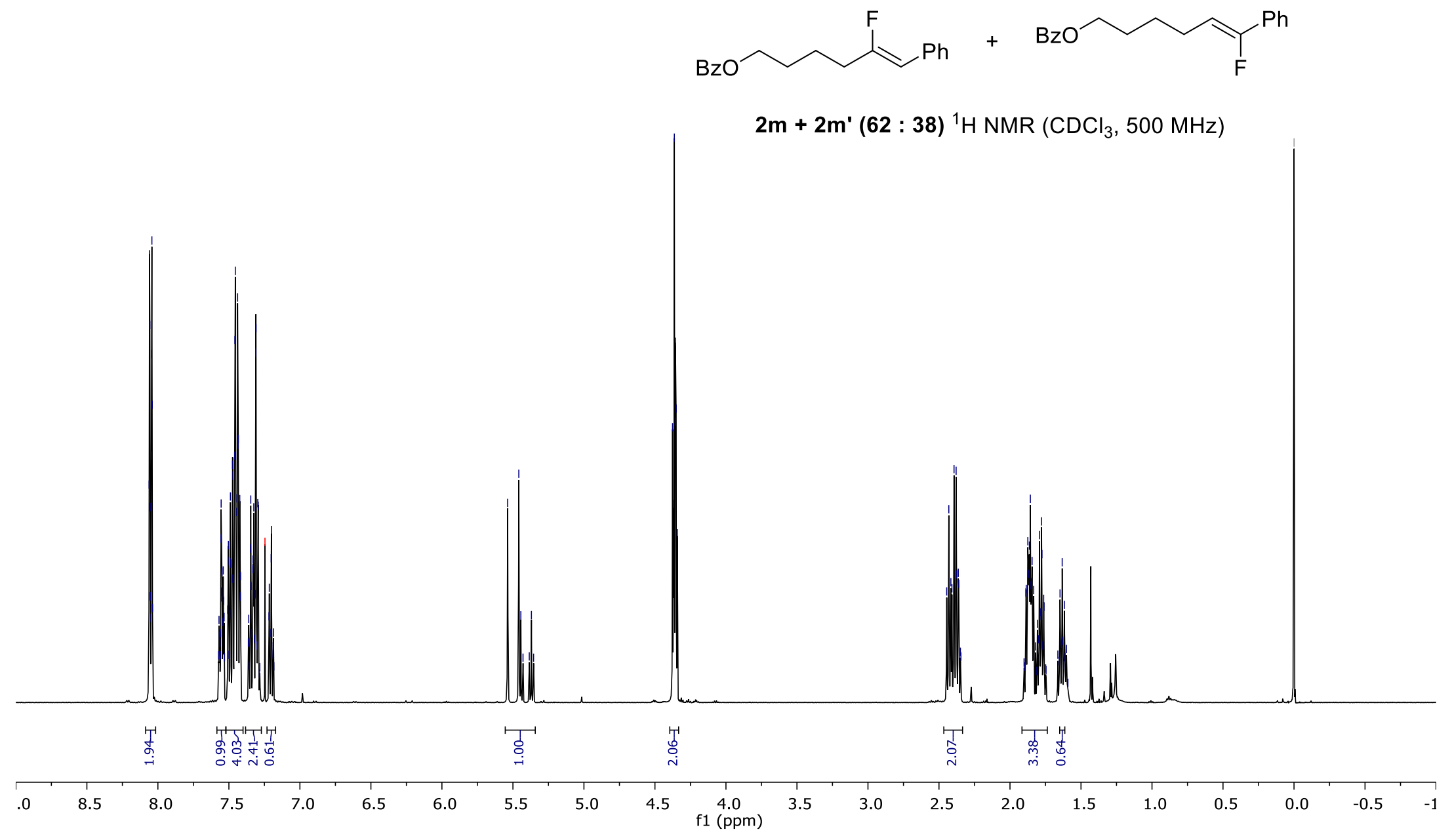




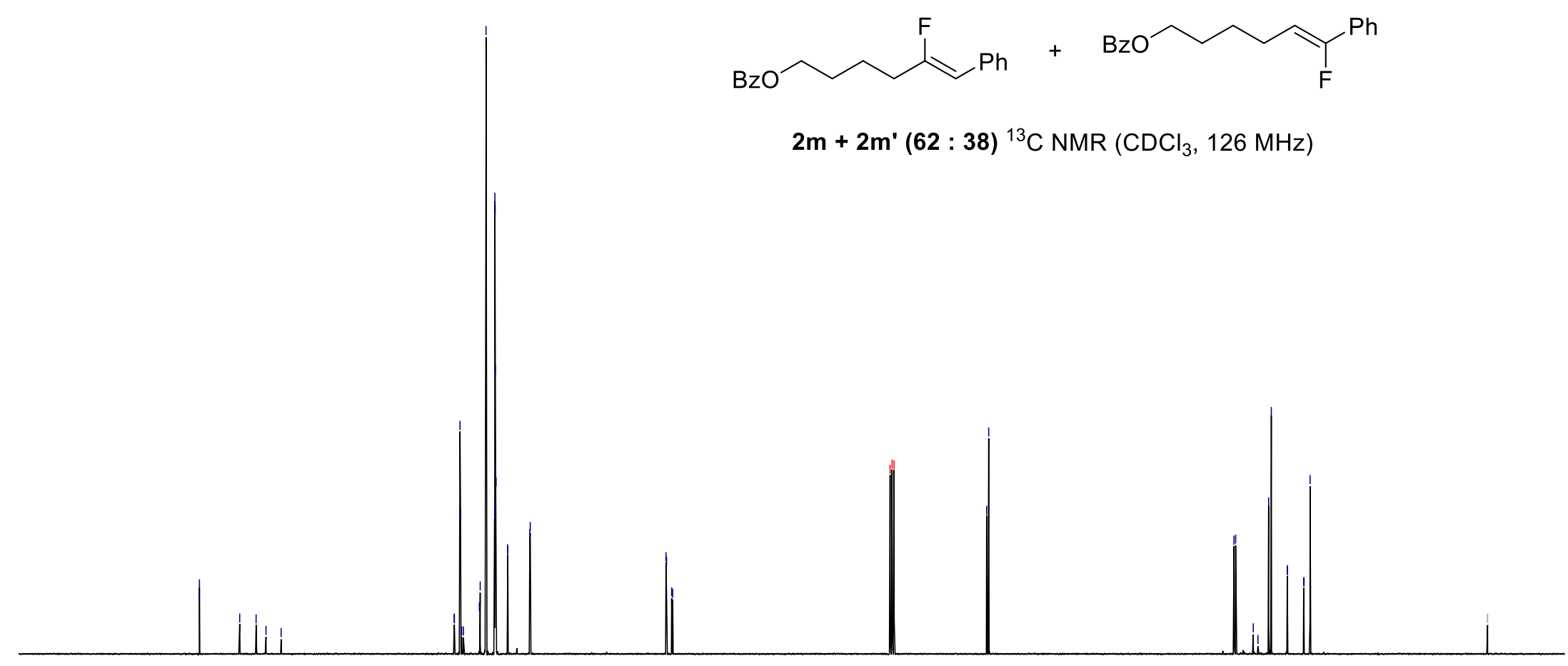

$90 \quad 180$

150

140

130

120

110

100

90

$80 \quad 70$

60

50

40

30 


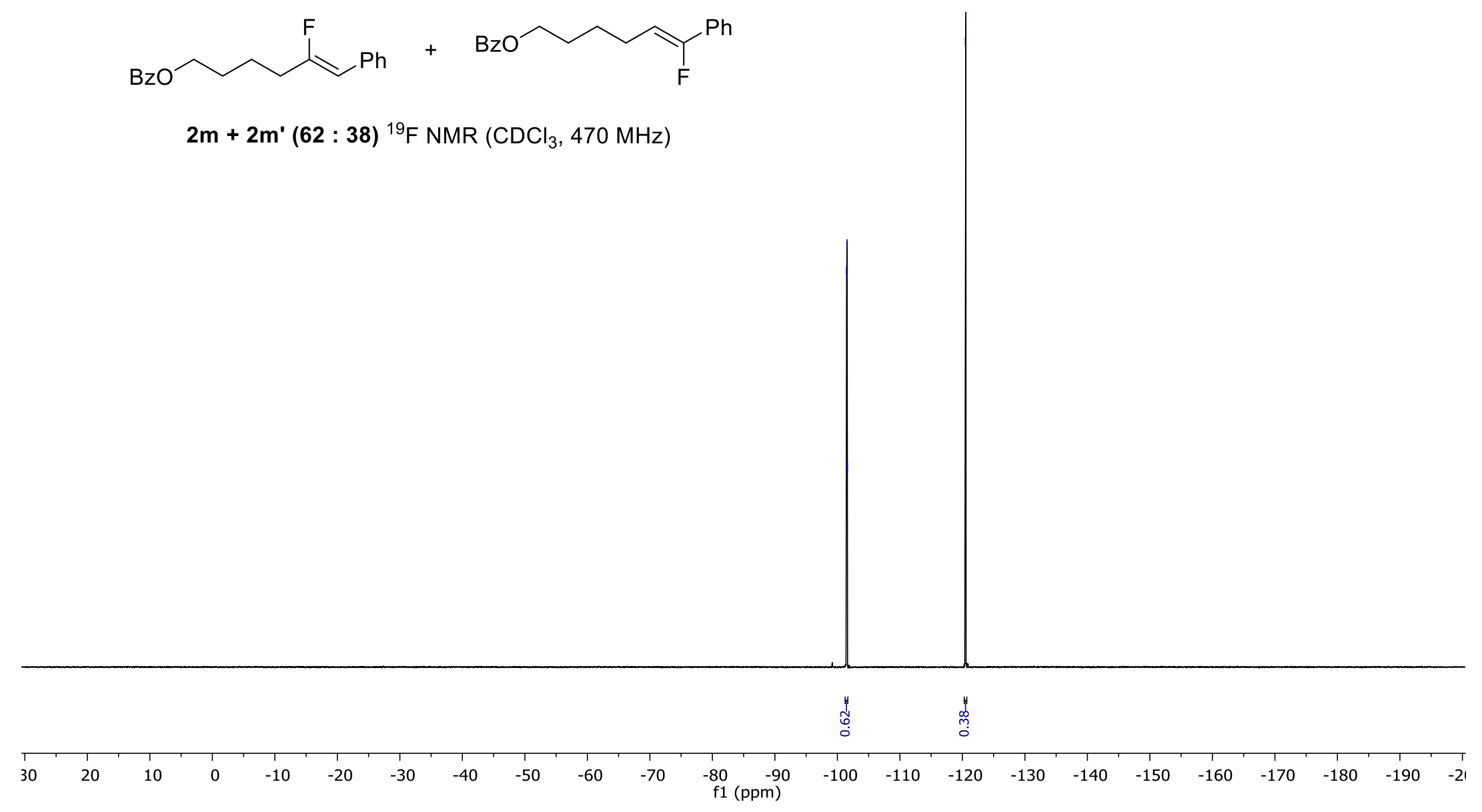

$2 m+2 m^{\prime}(62: 38){ }^{19} \mathrm{~F} \mathrm{NMR}\left(\mathrm{CDCl}_{3}, 470 \mathrm{MHz}\right)$ 


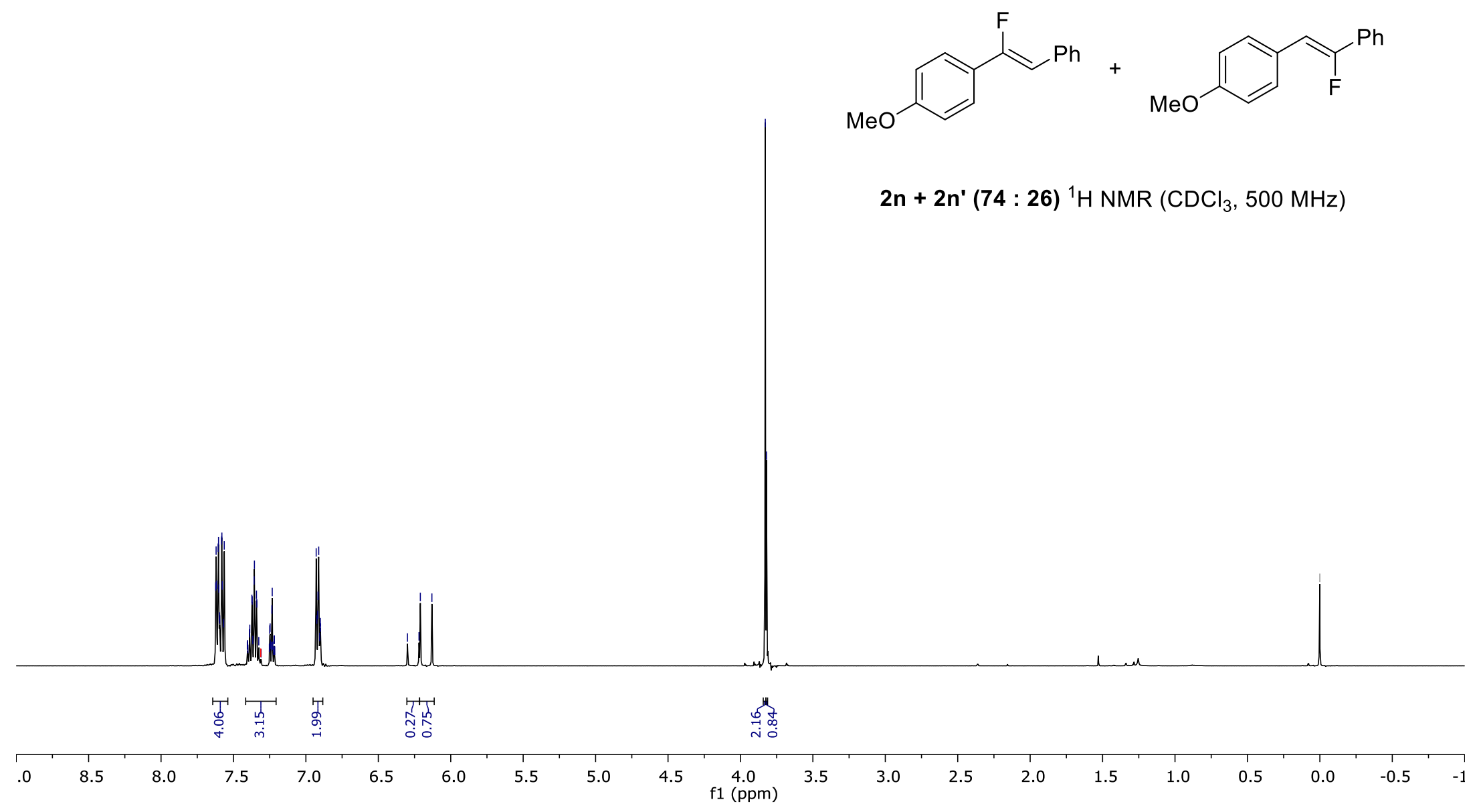




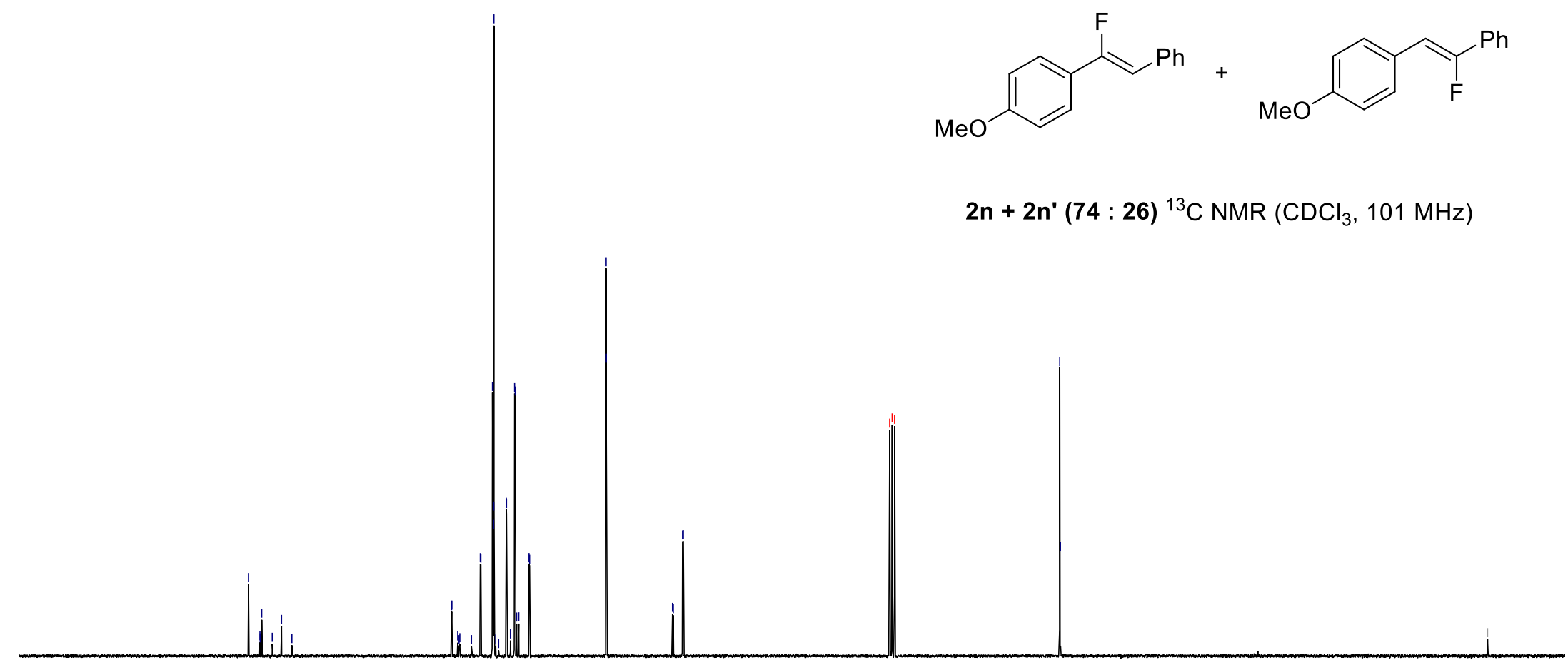

2n + 2n' (74: 26) ${ }^{13} \mathrm{C} \mathrm{NMR}\left(\mathrm{CDCl}_{3}, 101 \mathrm{MHz}\right)$

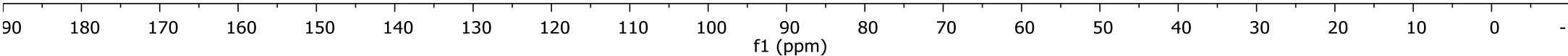




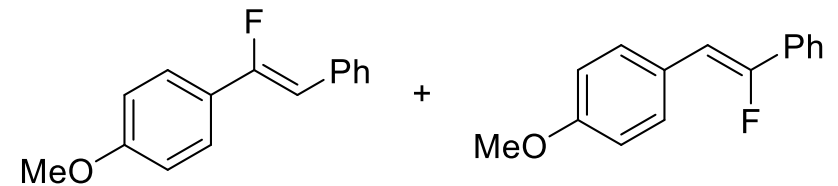

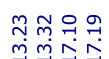

$\stackrel{9}{7}=$

$Y \gamma$

$2 n+2 n^{\prime}(74: 26){ }^{19} \mathrm{~F} \mathrm{NMR}\left(\mathrm{CDCl}_{3}, 470 \mathrm{MHz}\right)$

克兽

\begin{tabular}{llllllllllllllllllllllll}
\hline 30 & 20 & 10 & 0 & -10 & -20 & -30 & -40 & -50 & -60 & -70 & -80 & -90 & -100 & -110 & -120 & -130 & -140 & -150 & -160 & -170 & -180 & -190 & -2 \\
& & & & & & &
\end{tabular}



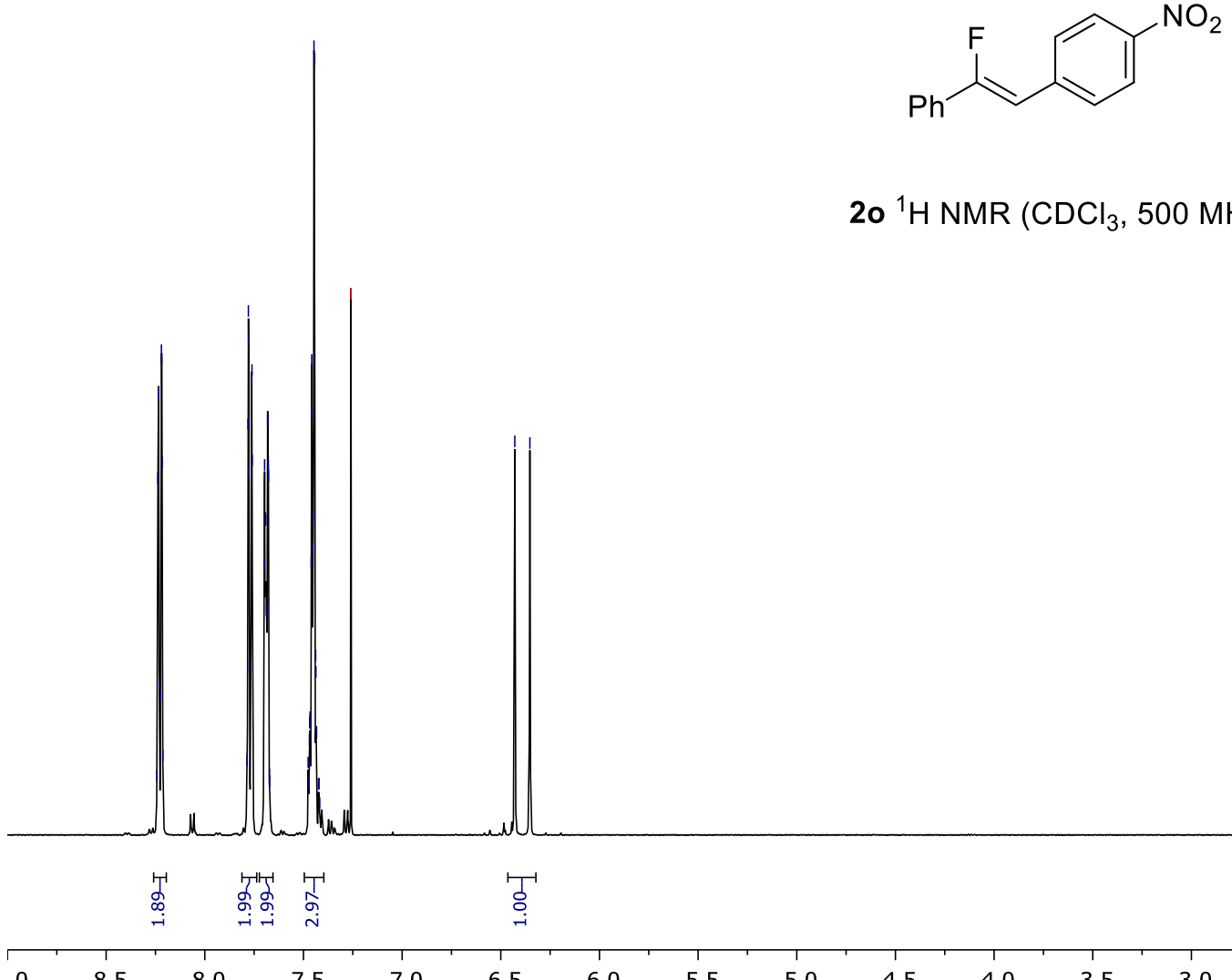

$20{ }^{1} \mathrm{H} \mathrm{NMR}\left(\mathrm{CDCl}_{3}, 500 \mathrm{MHz}\right)$

$.0 \quad 8.5$

8.0

7.5

6.5

$6.0 \quad 5.5$

5.0

4.5

4.0
$1(\mathrm{ppm})$

$3.5 \quad 3.0$

1.5

1.0

0.5

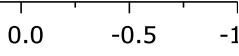




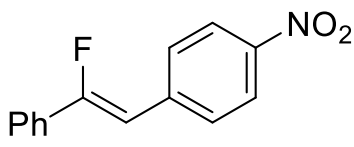

$20{ }^{19} \mathrm{~F} \mathrm{NMR}\left(\mathrm{CDCl}_{3}, 470 \mathrm{MHz}\right)$

Y

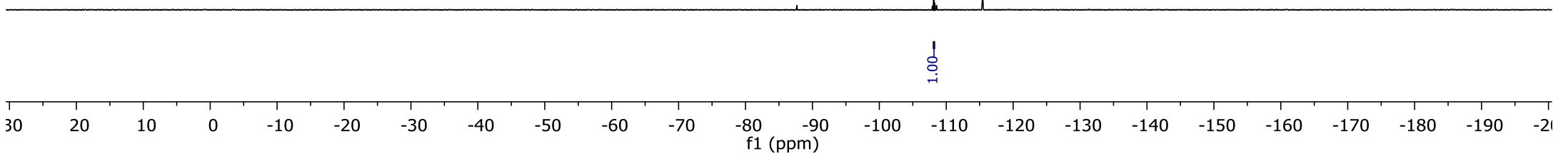




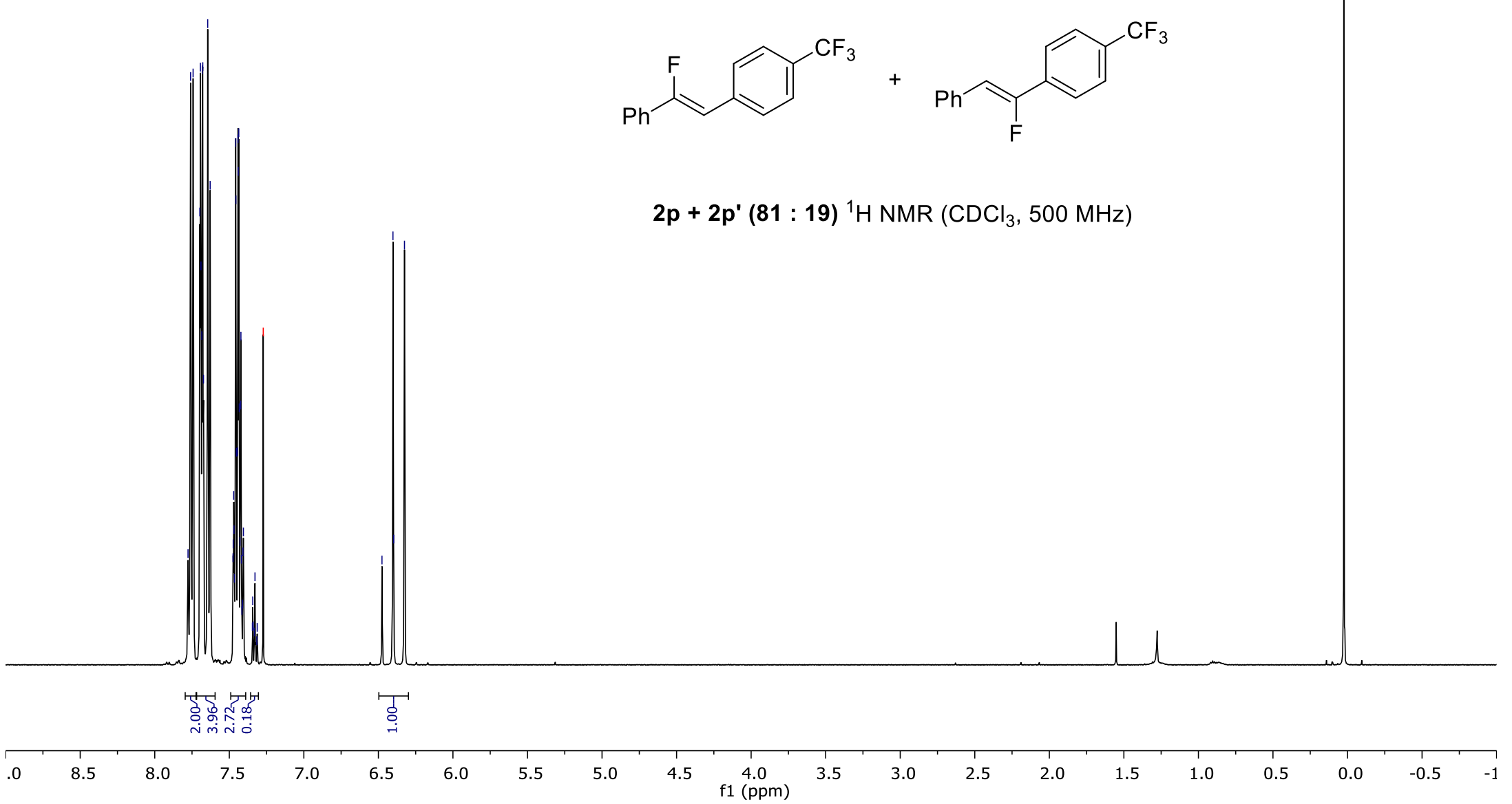


.

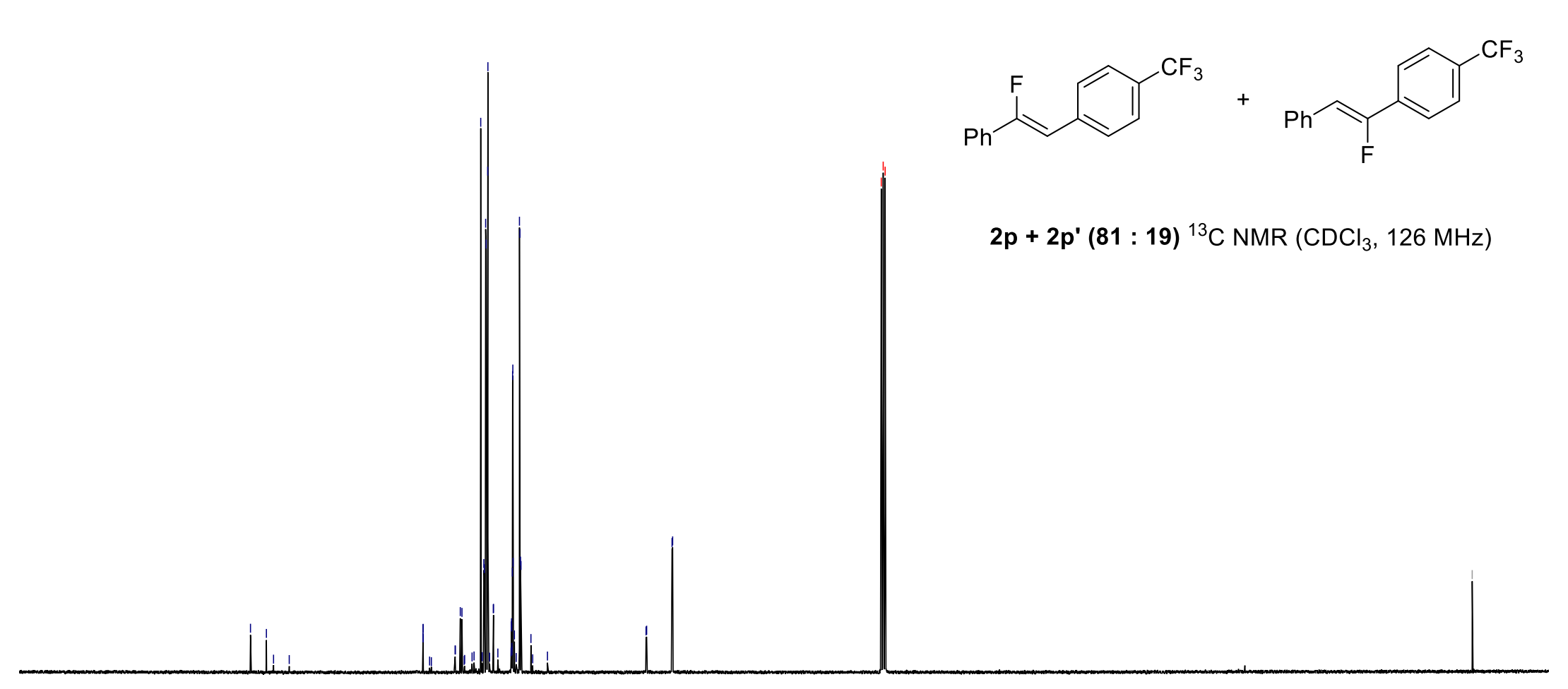

\begin{tabular}{|c|c|c|c|c|c|c|c|c|c|c|c|c|c|c|c|c|c|c|c|}
\hline 90 & 180 & 170 & 1,160 & 150 & 140 & 130 & 120 & 110 & 100 & 190 & 80 & 70 & & 50 & 40 & 30 & & 10 & 0 \\
\hline
\end{tabular}




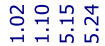

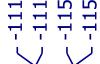

$\checkmark$

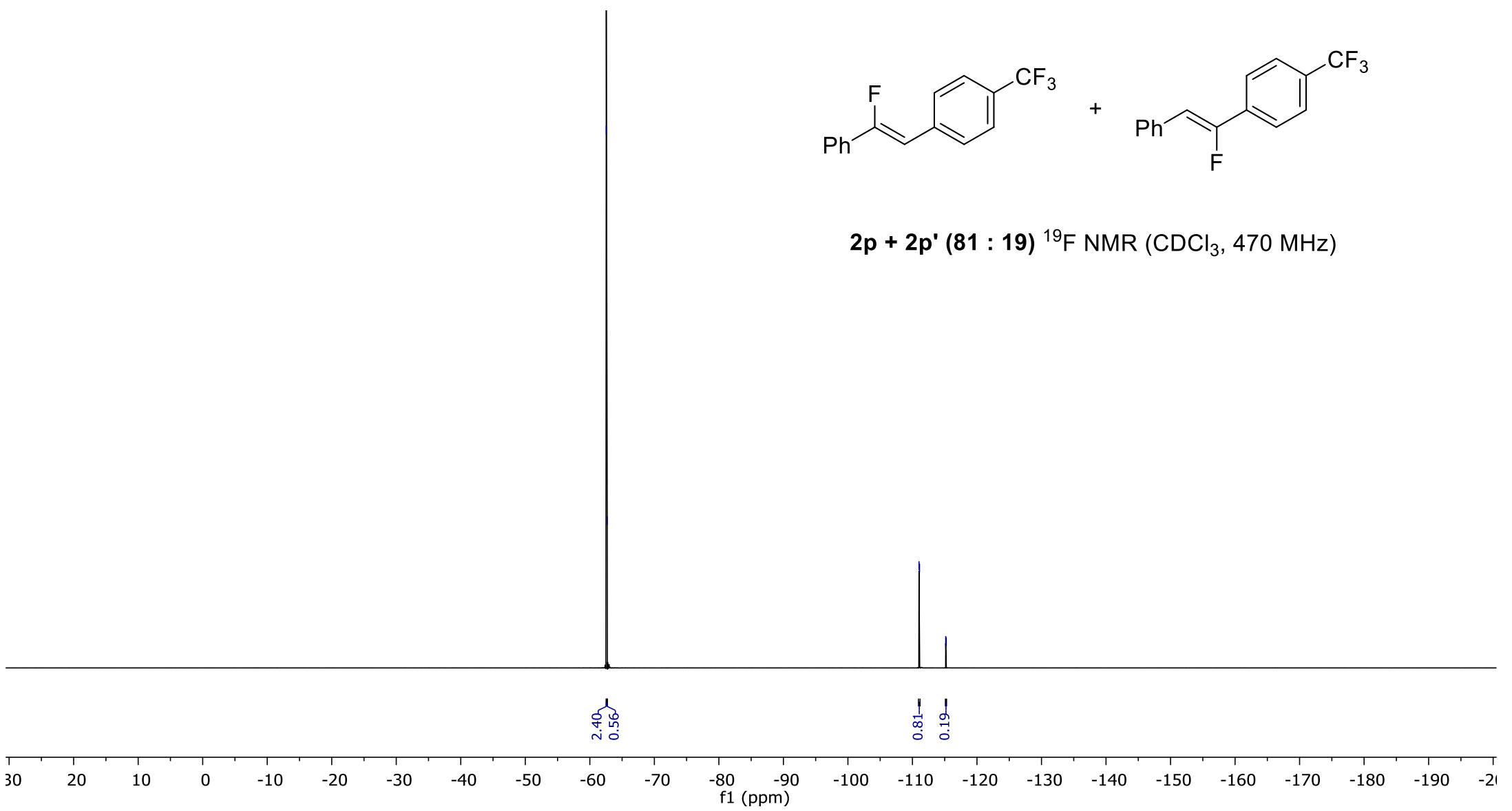

$2 p+2 p '(81: 19){ }^{19} \mathrm{~F} \mathrm{NMR}\left(\mathrm{CDCl}_{3}, 470 \mathrm{MHz}\right)$ 


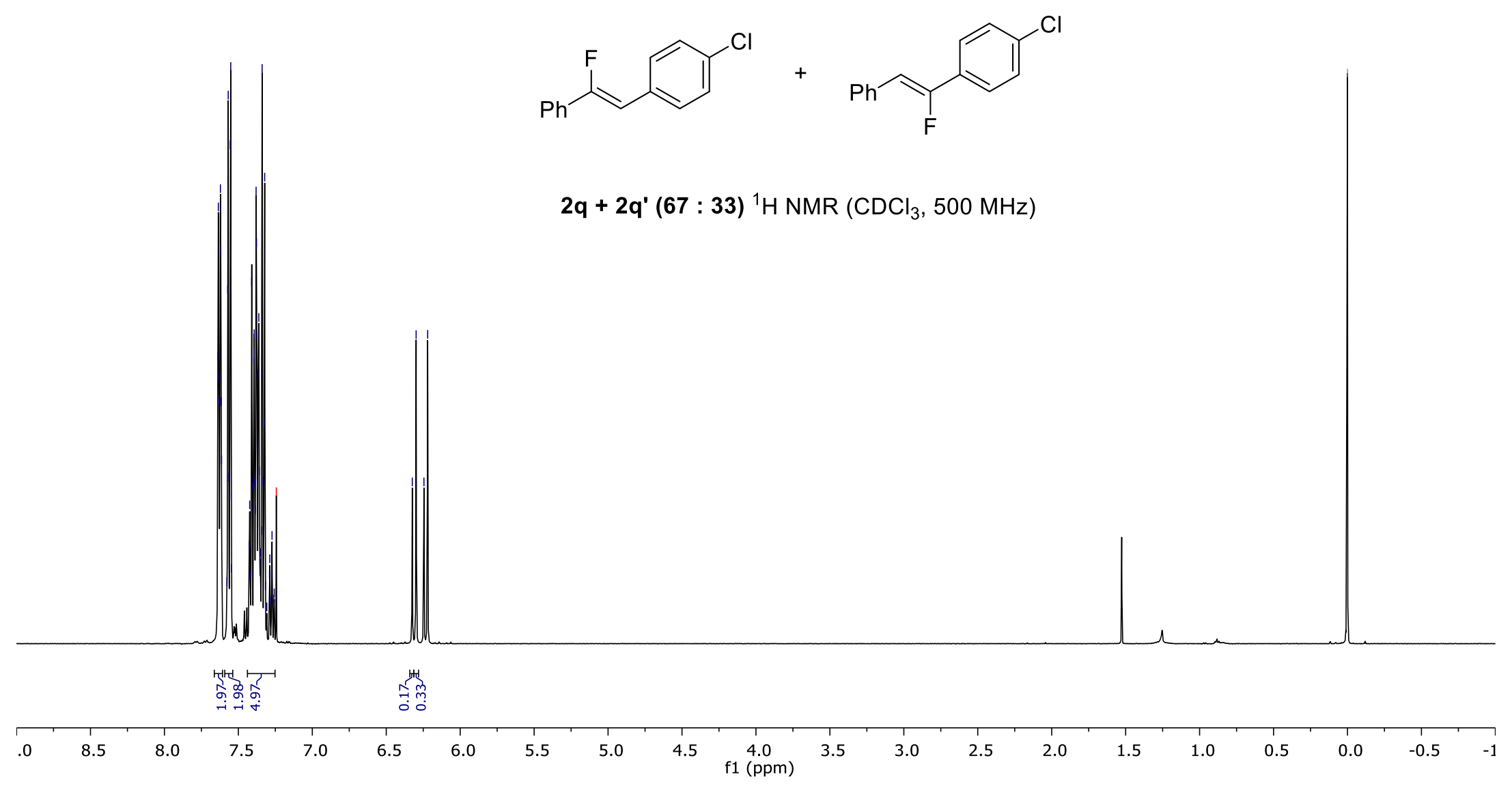



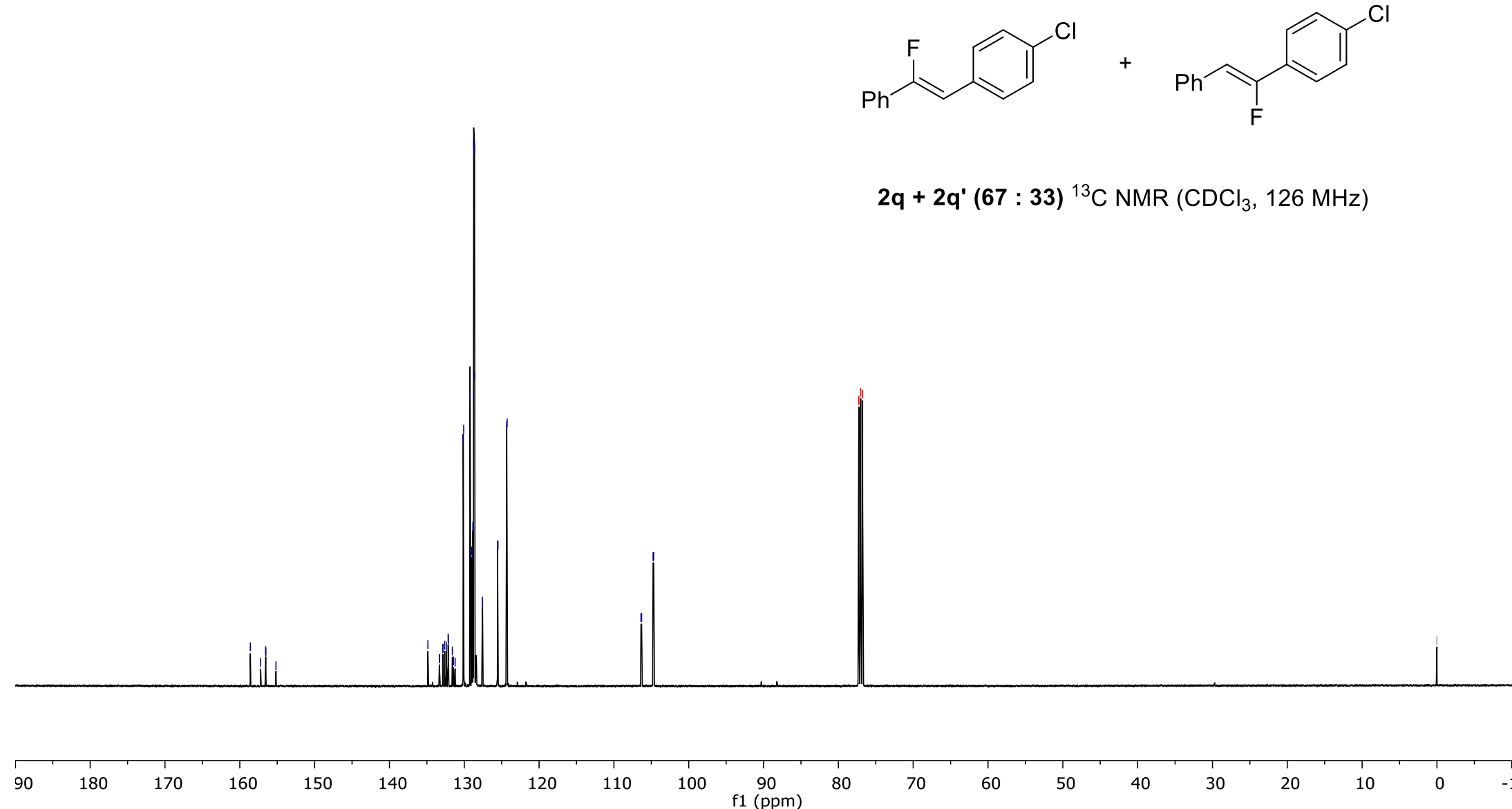


\section{欲星手量 \\ ำำำำ}

Yr

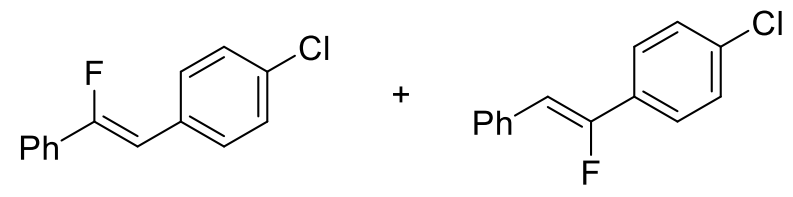

$2 q+2 q^{\prime}(67: 33){ }^{19} \mathrm{~F} \mathrm{NMR}\left(\mathrm{CDCl}_{3}, 470 \mathrm{MHz}\right)$

the

\begin{tabular}{lllllllllllllllllllllllllll}
\hline & 20 & 20 & 10 & 0 & -10 & -20 & -30 & -40 & -50 & -60 & -70 & -80 & -90 & -100 & -110 & -120 & -130 & -140 & -150 & -160 & -170 & -180 & -190 & -2
\end{tabular}




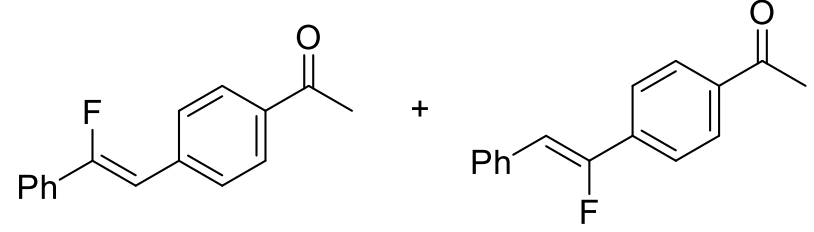

$2 r+2 r^{\prime}(84: 16){ }^{1} \mathrm{H} \mathrm{NMR}\left(\mathrm{CDCl}_{3}, 500 \mathrm{MHz}\right)$

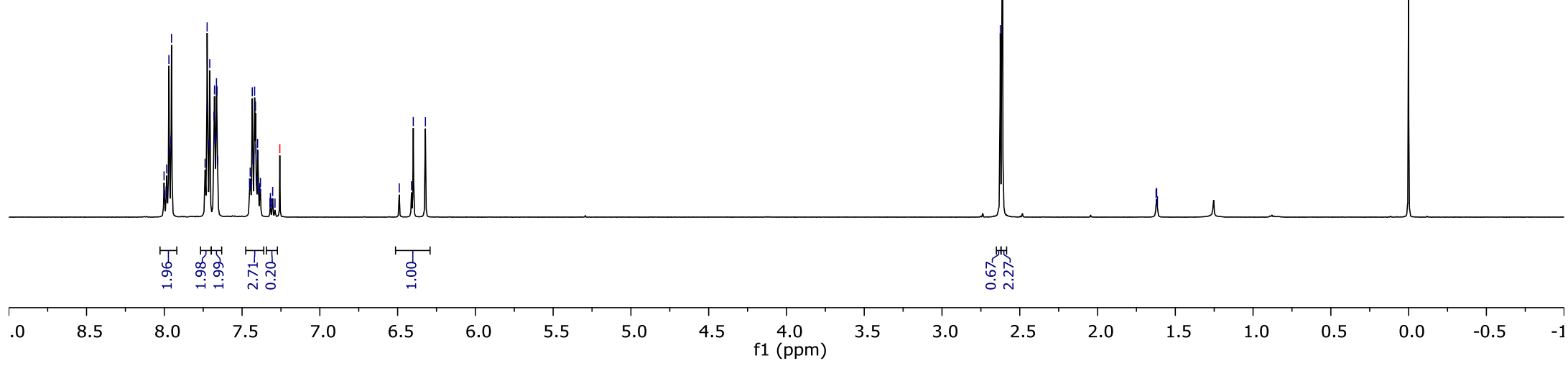




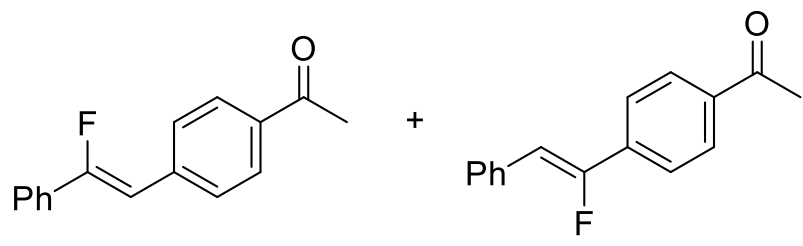

$2 r+2 r^{\prime}(84: 16){ }^{13} \mathrm{C} \mathrm{NMR}\left(\mathrm{CDCl}_{3}, 126 \mathrm{MHz}\right)$

$10 \quad 200-190$

$180 \quad 170$

160

$150 \quad 140$

$130 \quad 120$

110

100

$90 \quad 80 \quad 70$

$60 \quad 50$

$40 \quad 30$ 


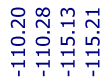

Y $Y$

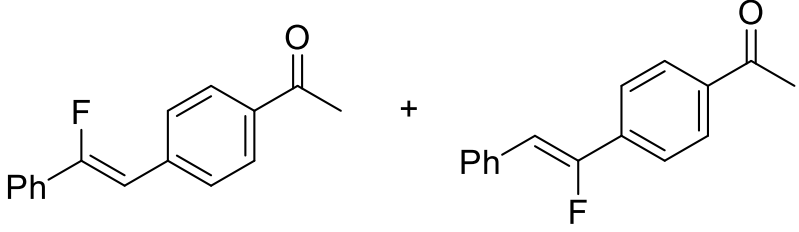

$2 r+2 r^{\prime}(84: 16){ }^{19} \mathrm{~F} \mathrm{NMR}\left(\mathrm{CDCl}_{3}, 470 \mathrm{MHz}\right)$

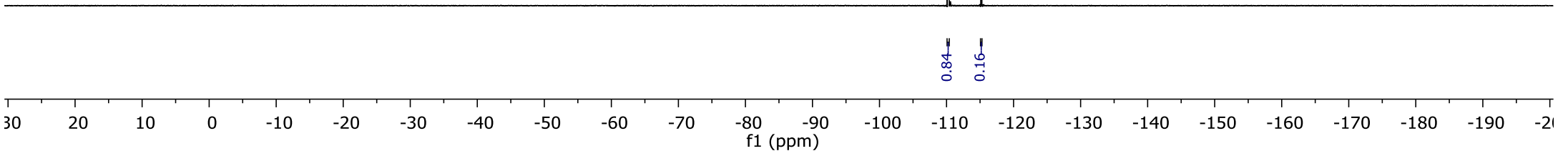




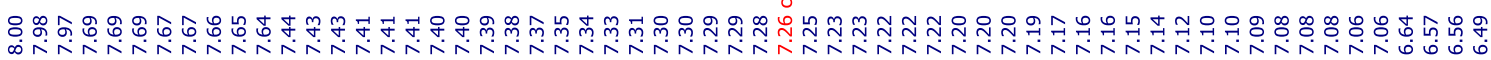

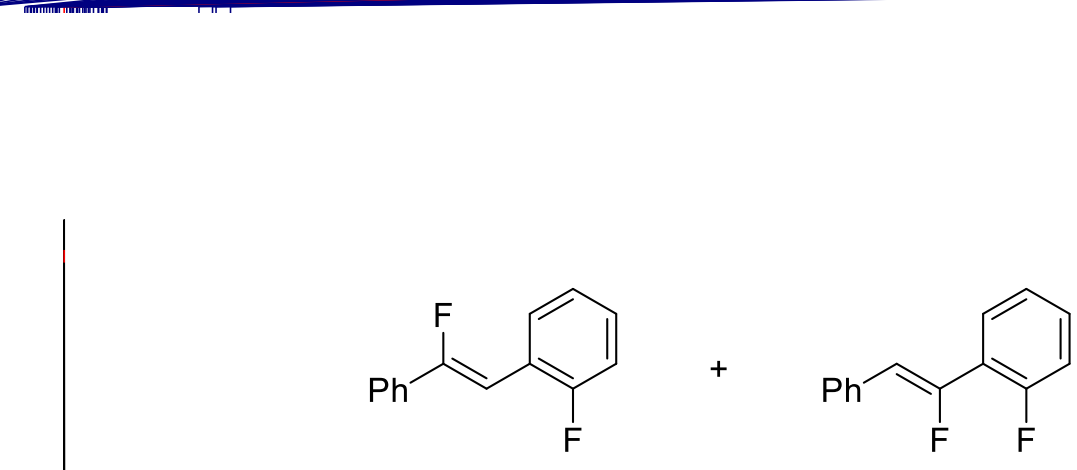

2s + 2s' (77: 23) ${ }^{1} \mathrm{H} \mathrm{NMR}\left(\mathrm{CDCl}_{3}, 500 \mathrm{MHz}\right)$

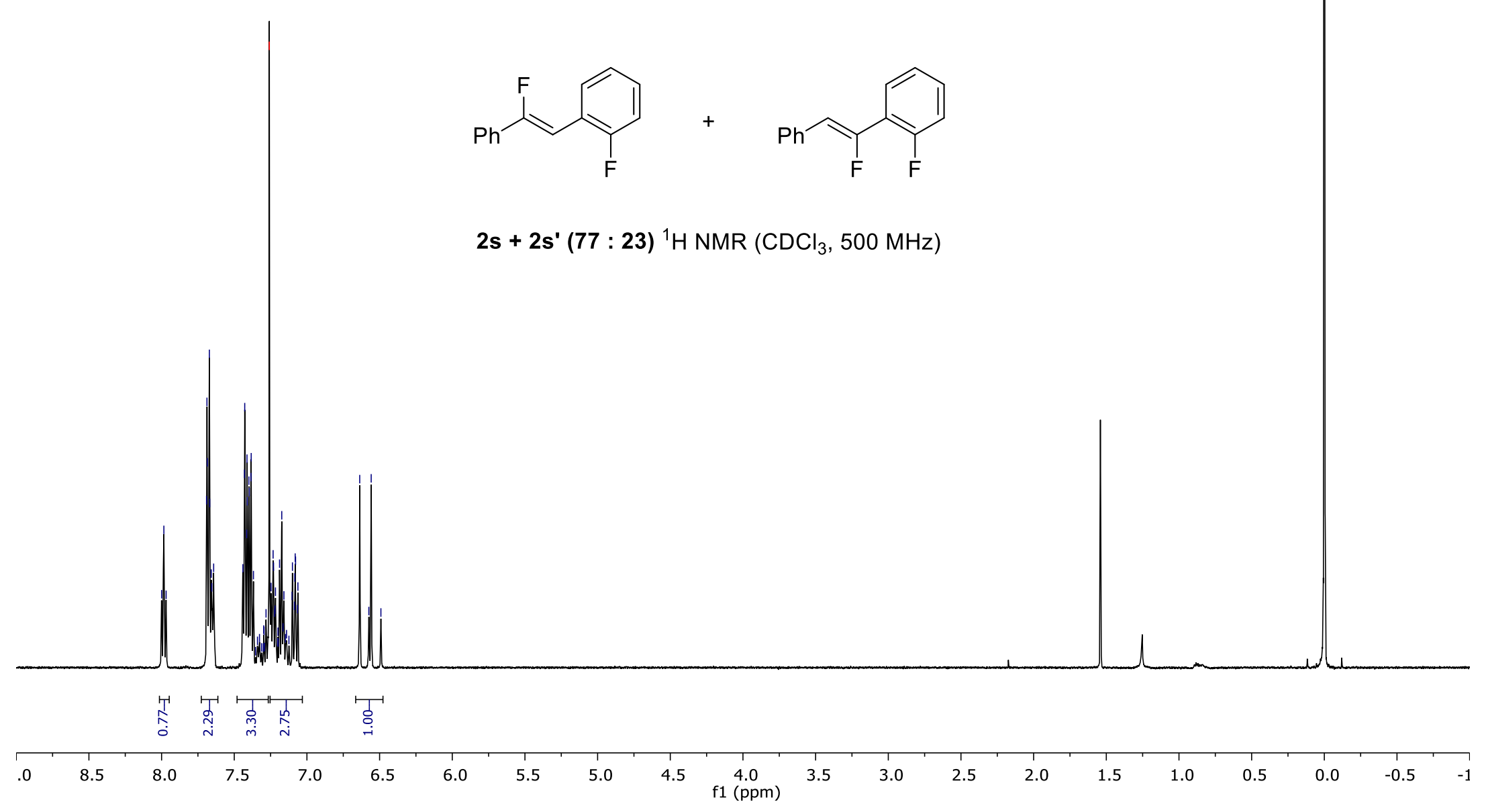


等

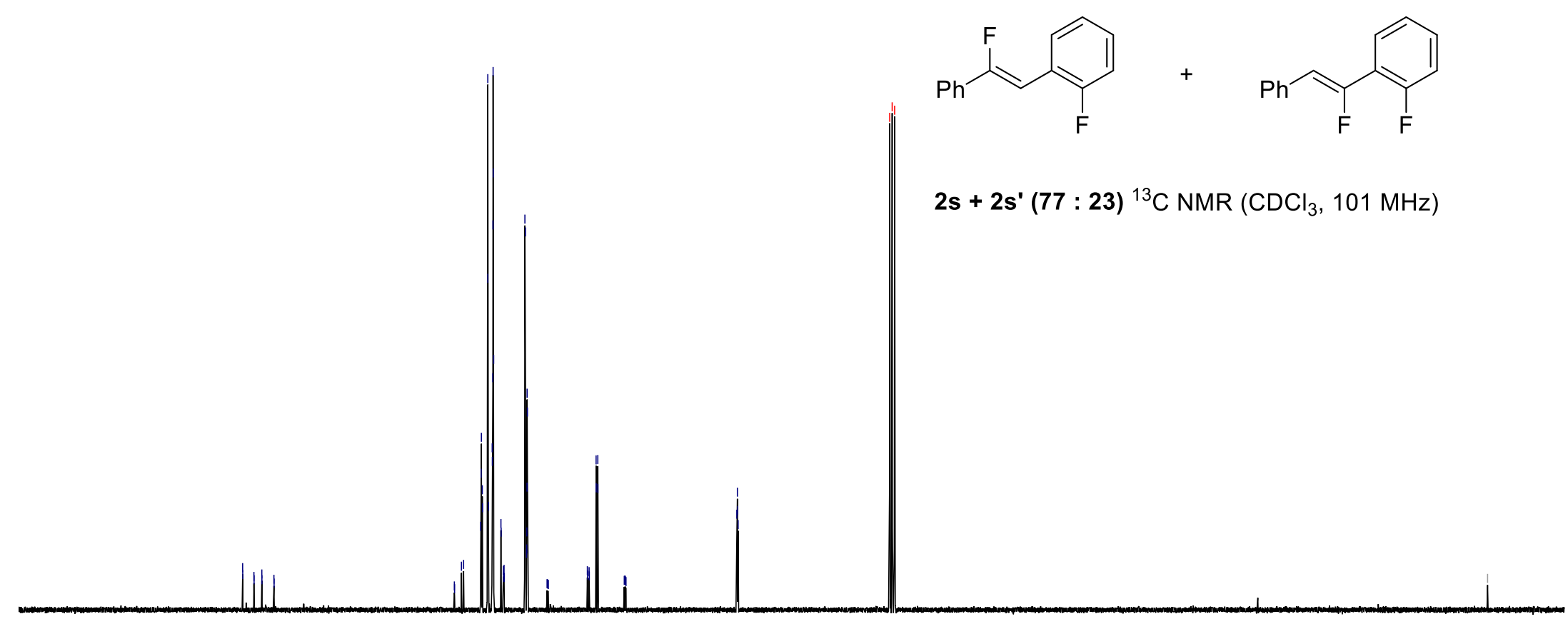

$90 \quad 180 \quad 170 \quad 160 \quad 150 \quad 140 \quad 130 \quad 120$

100

90

$80 \quad 70 \quad 60$

50

40

30

20

$10 \quad 0$ 


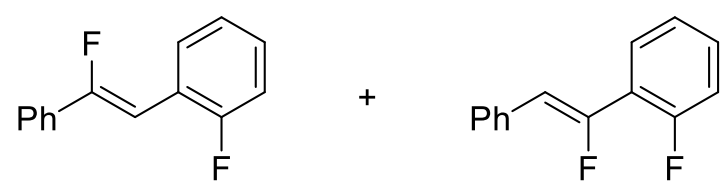

2s + 2s' (77: 23) ${ }^{19} \mathrm{~F} \mathrm{NMR}\left(\mathrm{CDCl}_{3}, 470 \mathrm{MHz}\right)$

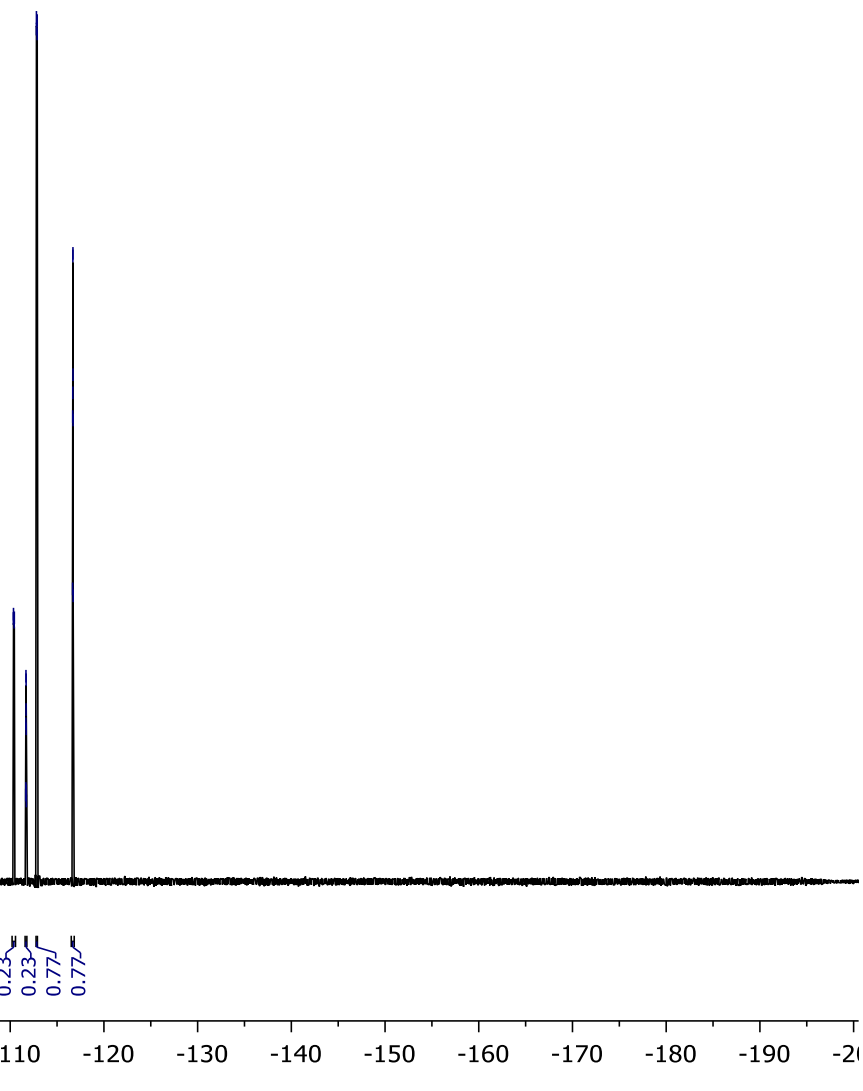

\begin{tabular}{rlllllllllllllllllllllll}
\hline 30 & 20 & 10 & 0 & -10 & -20 & -30 & -40 & -50 & -60 & -70 & -80 & -90 & -100 & -110 & -120 & -130 & -140 & -150 & -160 & -170 & -180 & -190 & -2
\end{tabular}




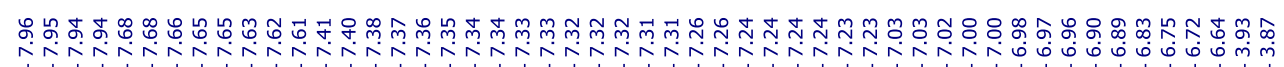

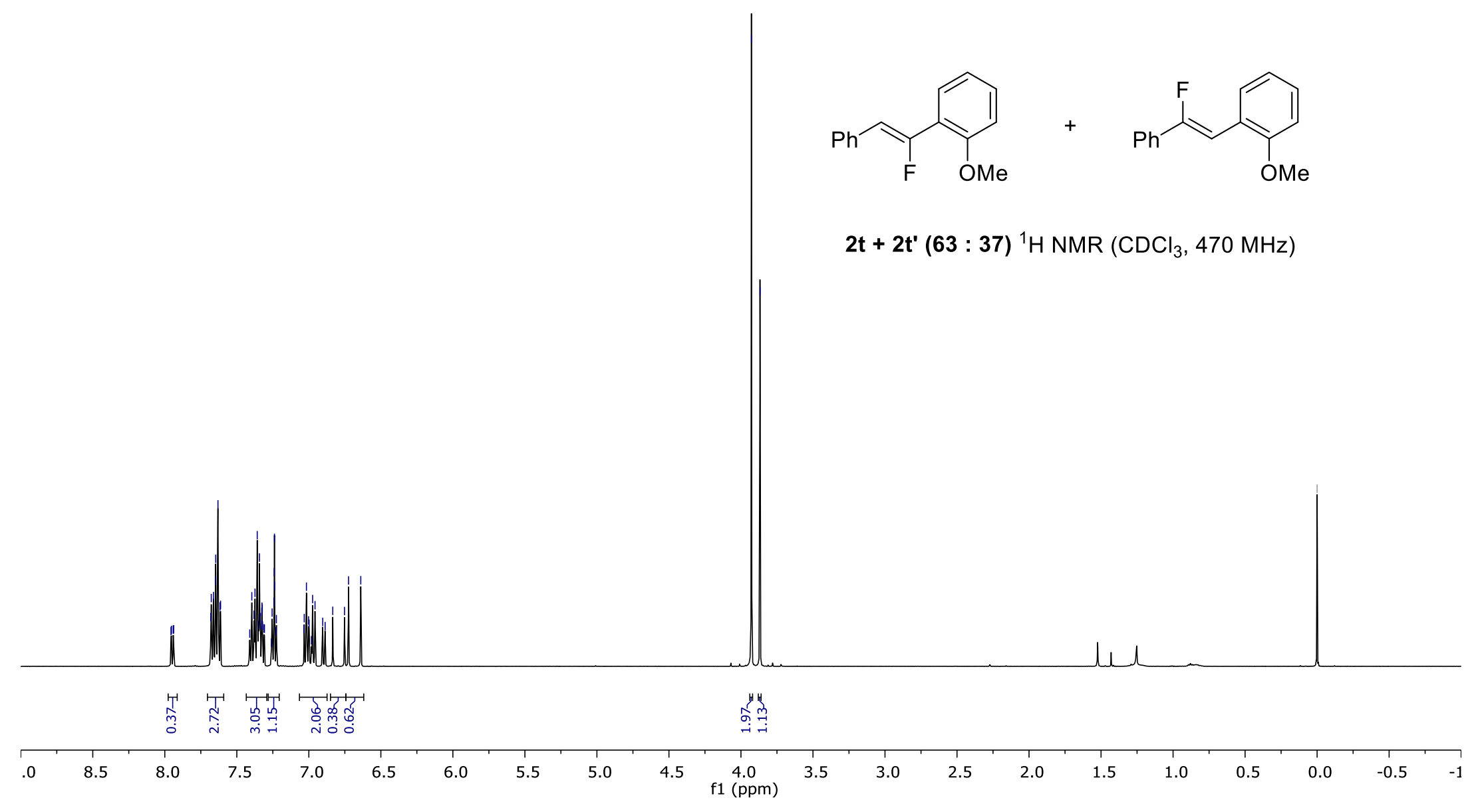



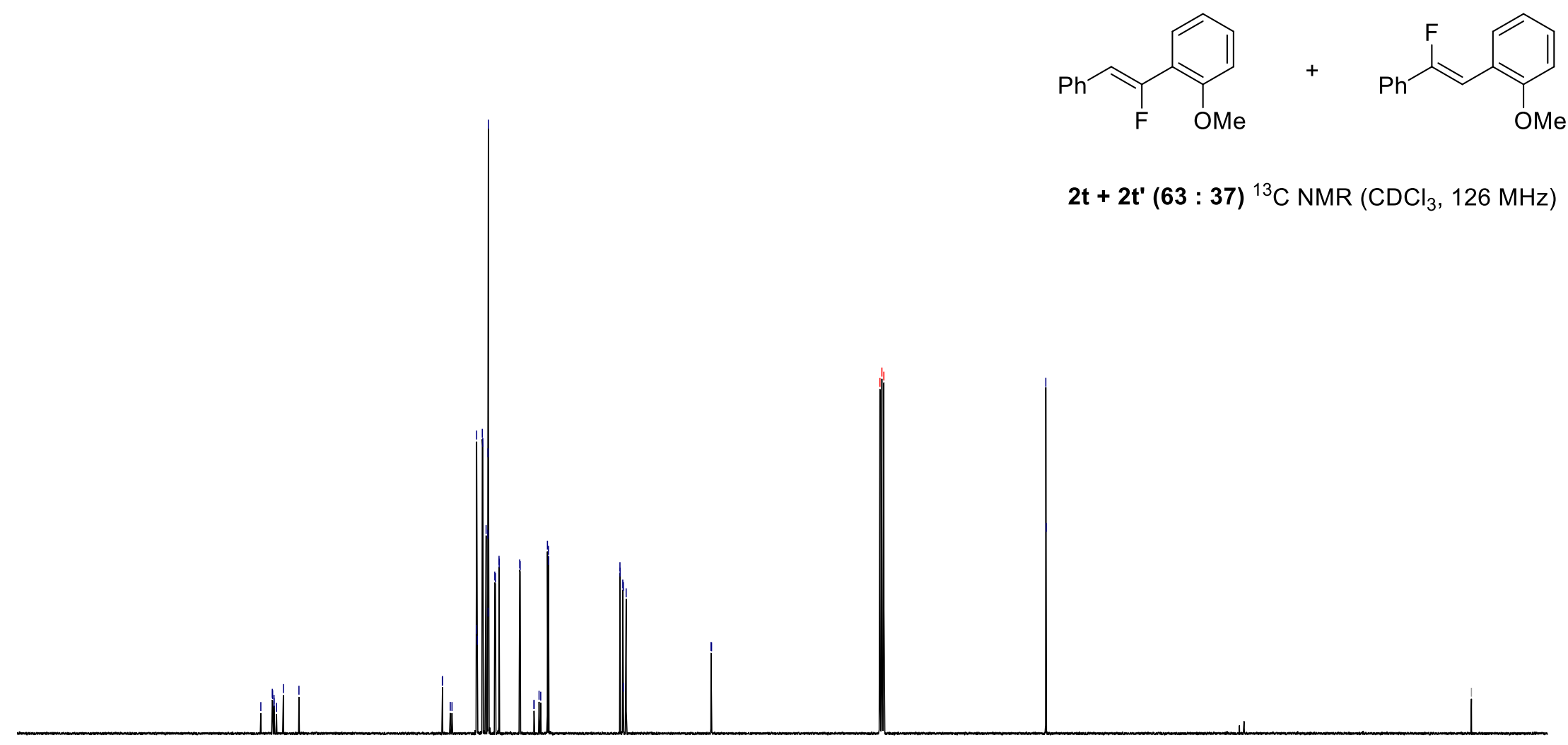

$90 \quad 180 \quad 170 \quad 160 \quad 150 \quad 140 \quad 130 \quad 120$

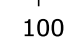

90

$80 \quad 70$

60

50

40

30 


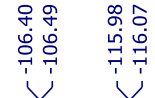

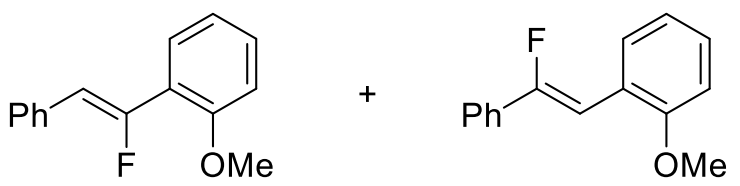

$2 t+2 t^{\prime}(63: 37){ }^{19} \mathrm{~F} \mathrm{NMR}\left(\mathrm{CDCl}_{3}, 470 \mathrm{MHz}\right)$

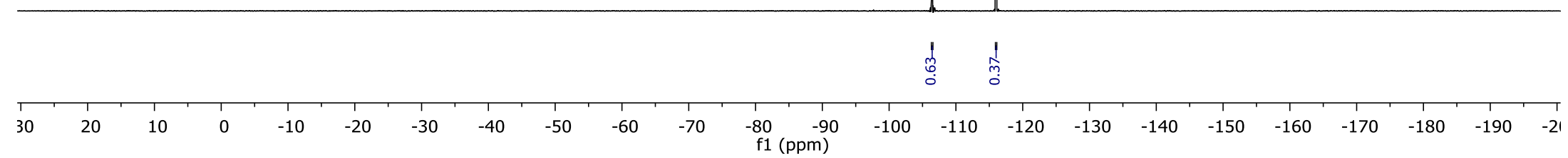




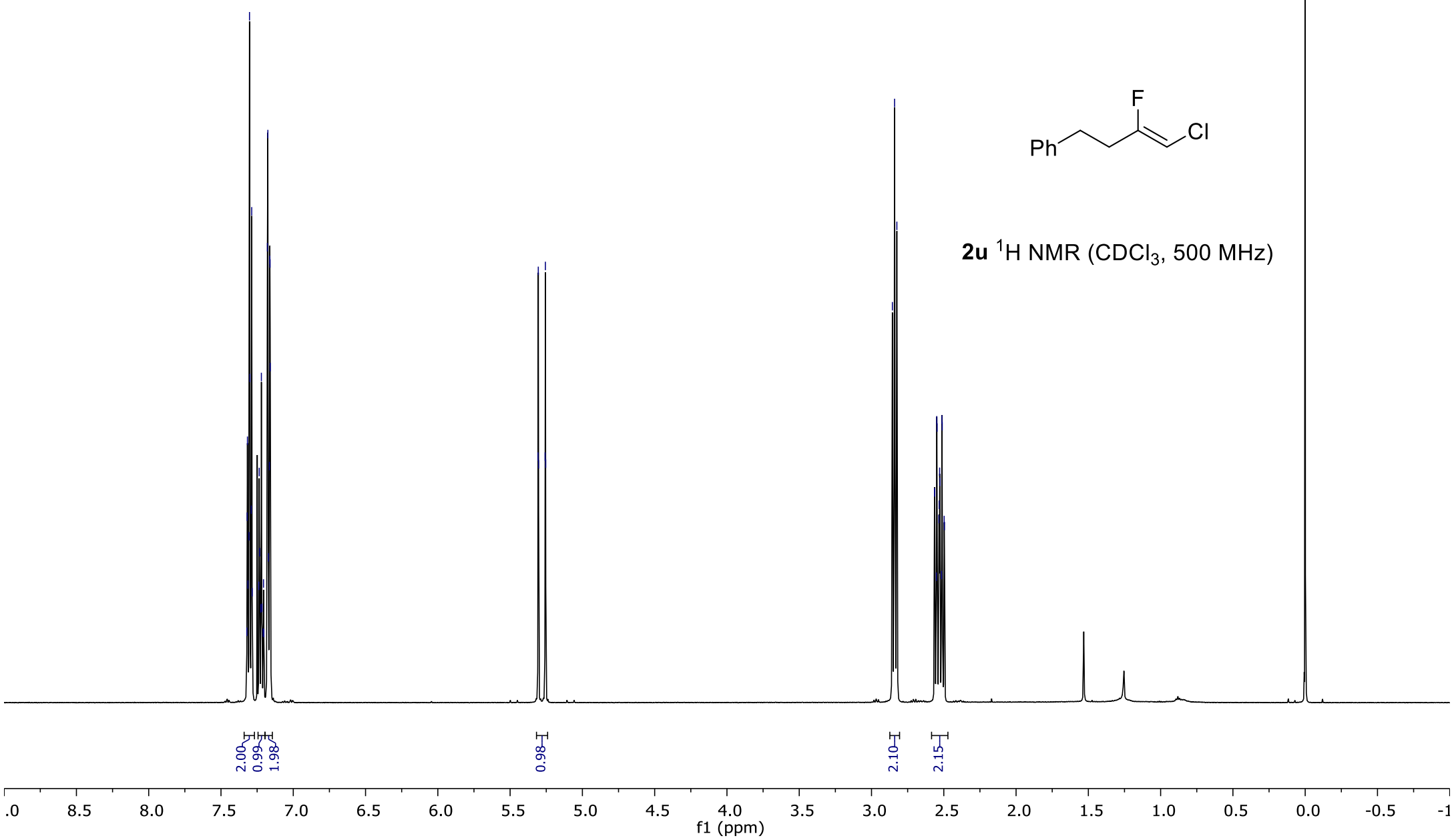



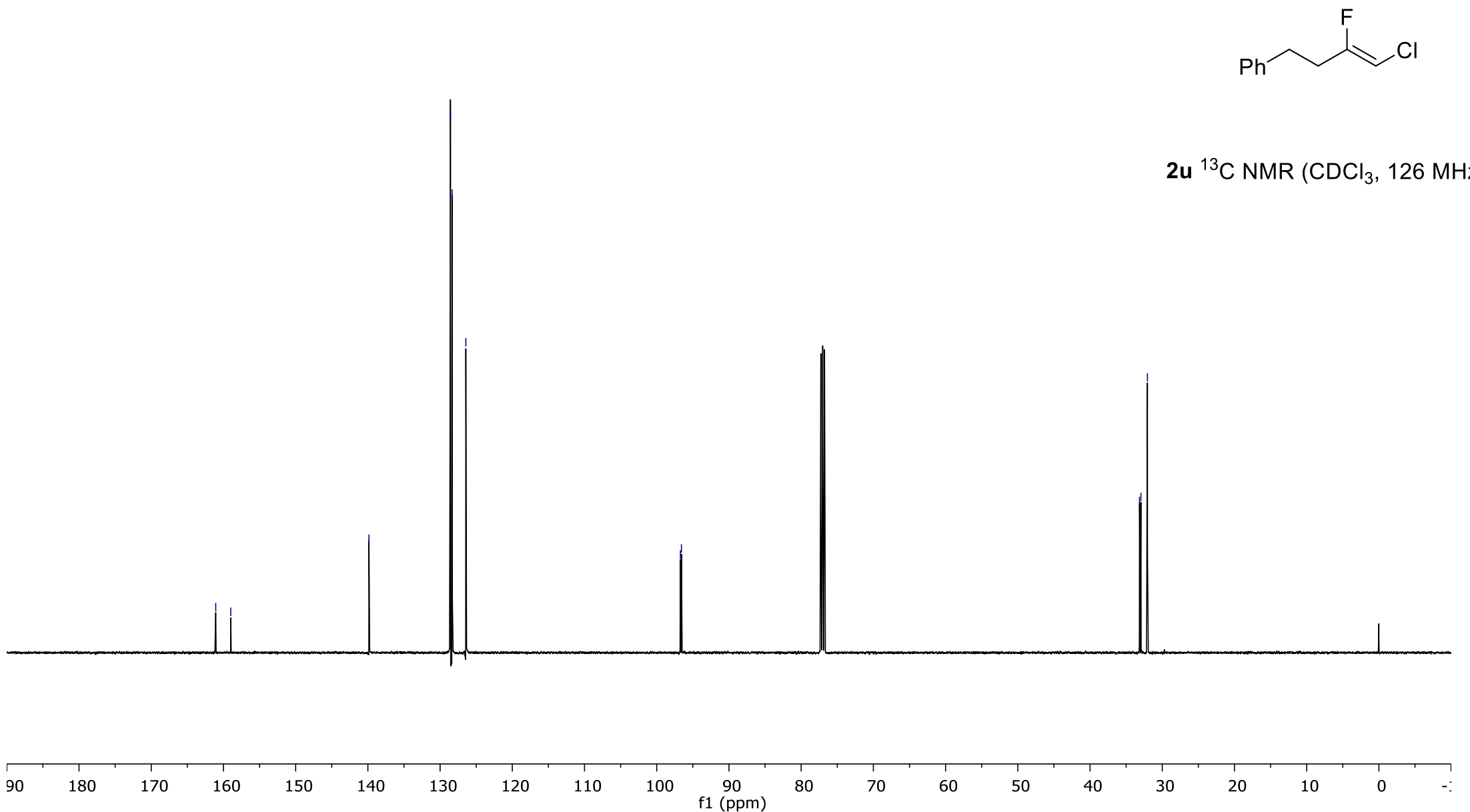
용ㅎํㅇํำ영

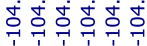

瓷

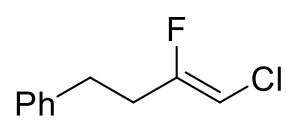

$2 \mathbf{u}^{19} \mathrm{~F} \mathrm{NMR}\left(\mathrm{CDCl}_{3}, 470 \mathrm{MHz}\right)$

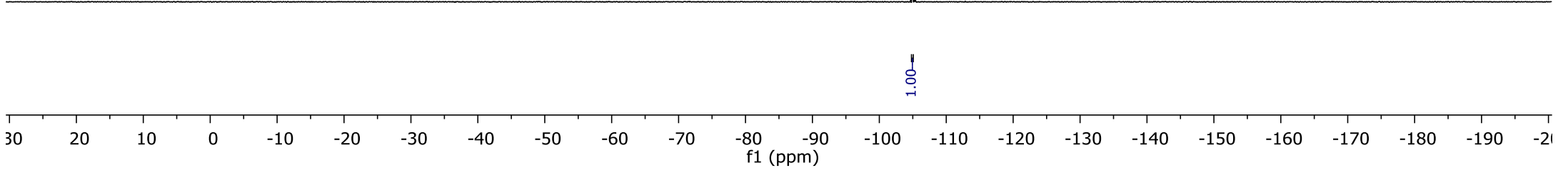




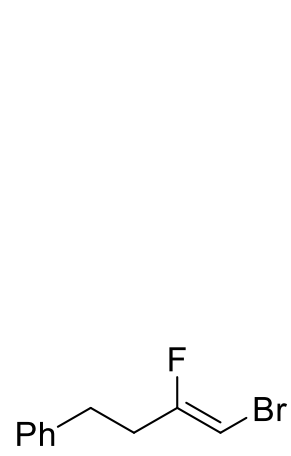

2v ${ }^{1} \mathrm{H}$ NMR $\left(\mathrm{CDCl}_{3}, 500 \mathrm{MHz}\right)$

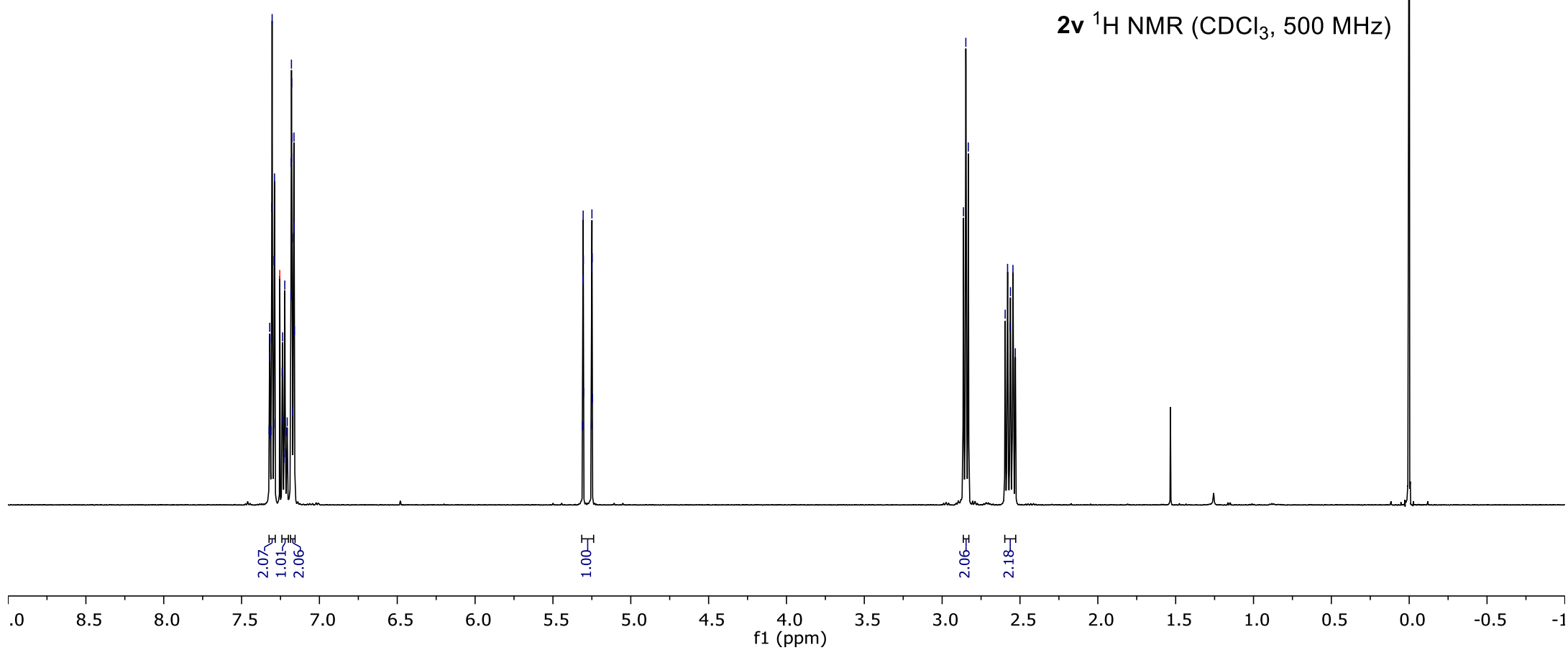



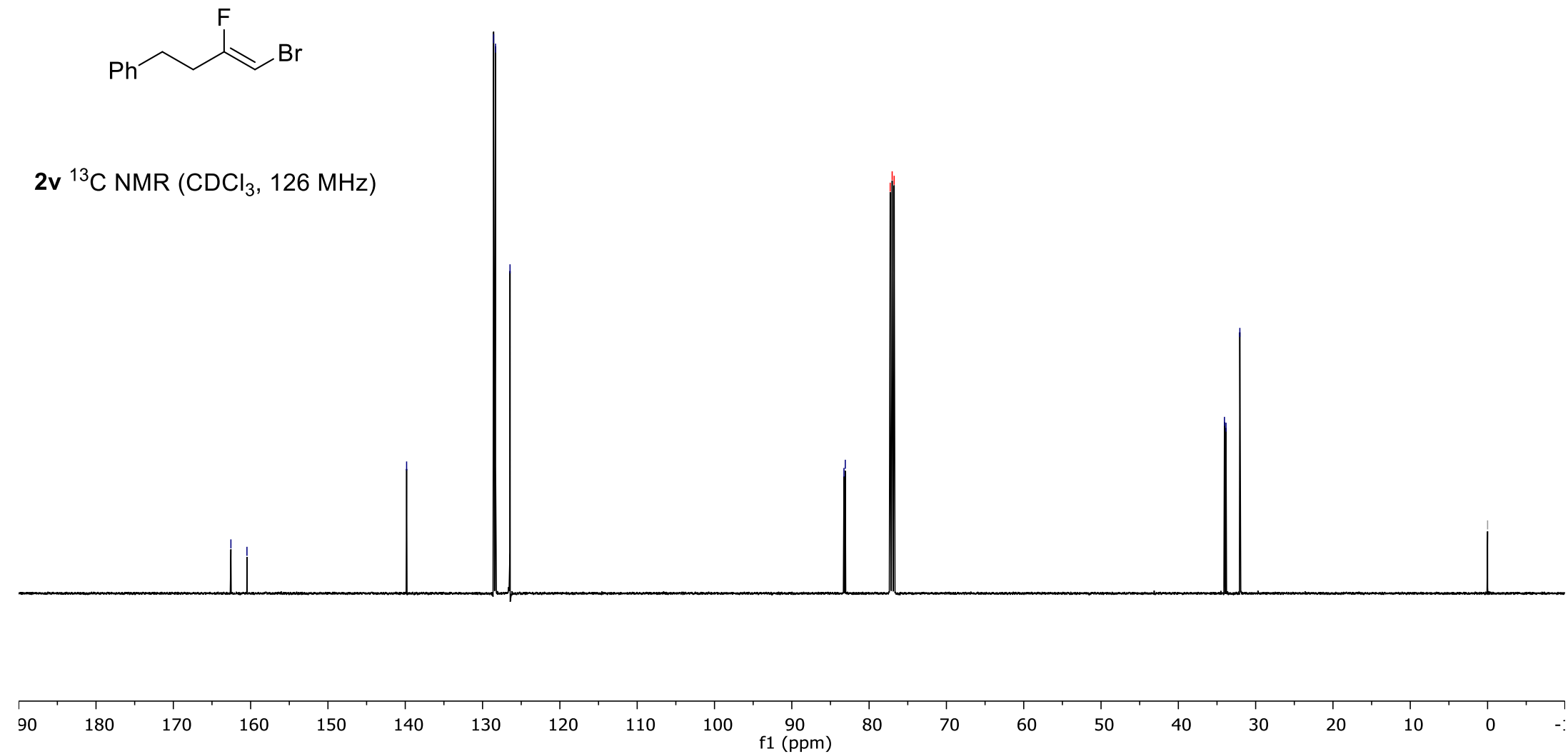


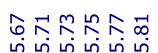

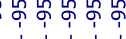

ए

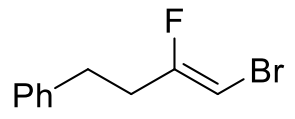

\section{2v ${ }^{19} \mathrm{~F} \mathrm{NMR}\left(\mathrm{CDCl}_{3}, 470 \mathrm{MHz}\right)$}

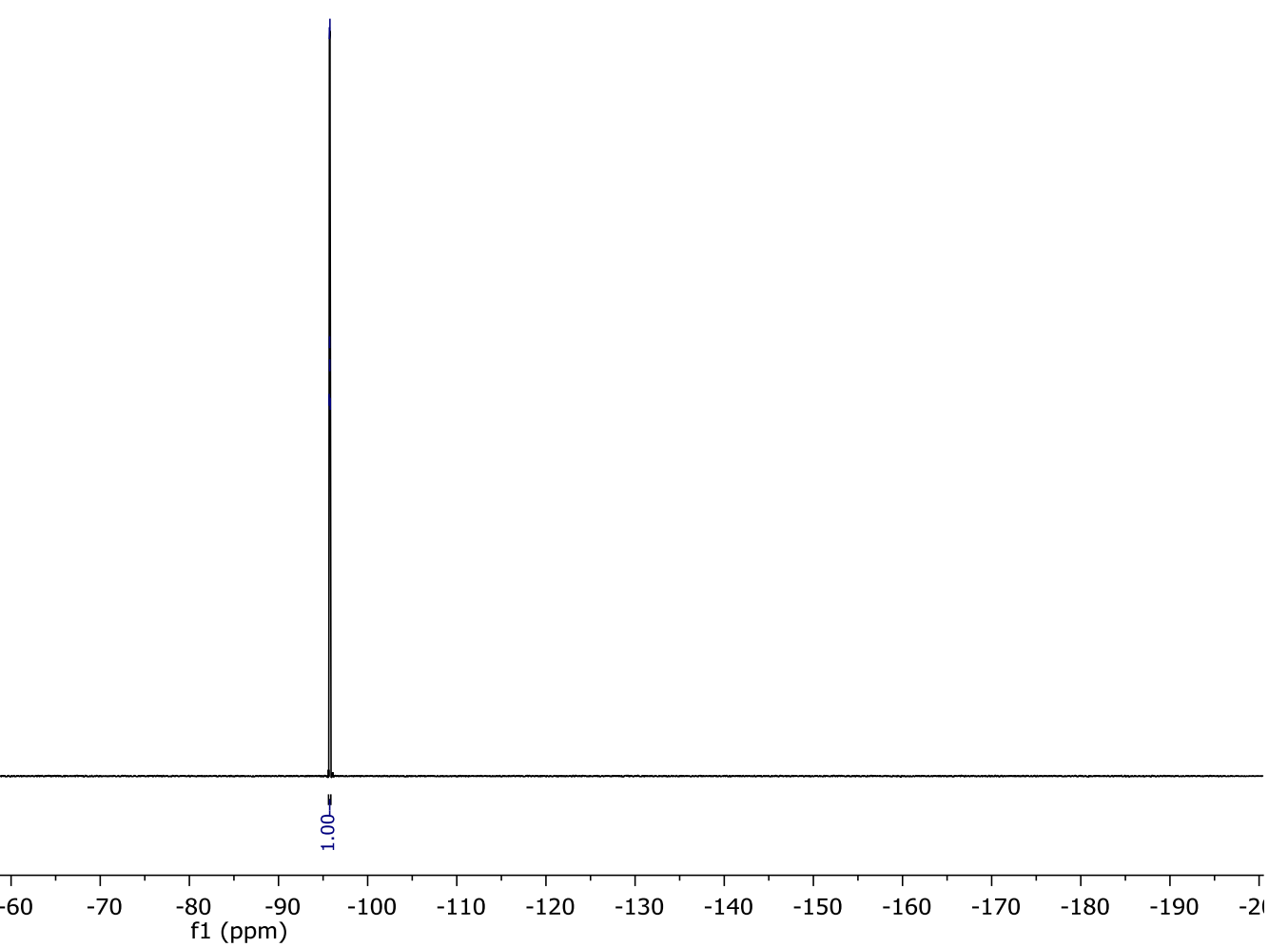




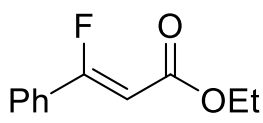

2w ${ }^{1} \mathrm{H} \mathrm{NMR}\left(\mathrm{CDCl}_{3}, 500 \mathrm{MHz}\right)$
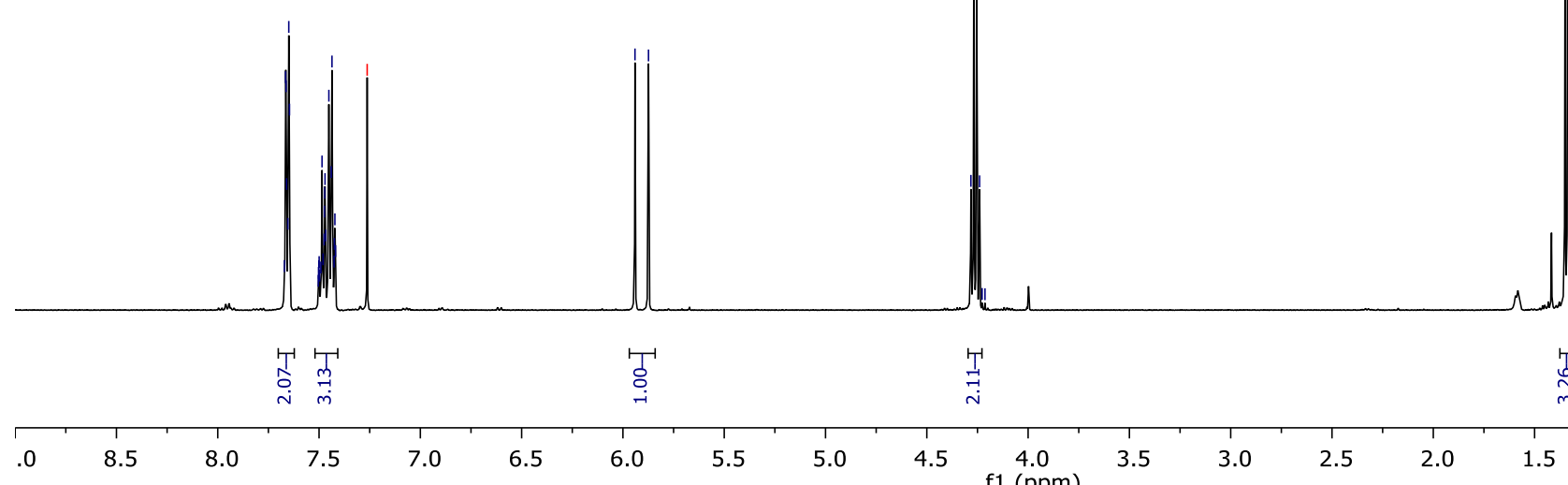


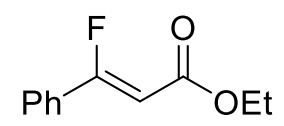

2w ${ }^{19} \mathrm{~F} \mathrm{NMR}\left(\mathrm{CDCl}_{3}, 470 \mathrm{MHz}\right)$

b

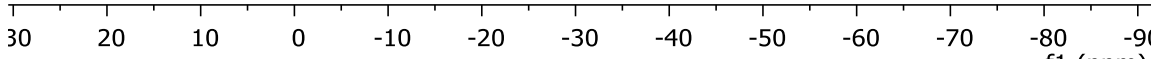
-80
f1
$(\mathrm{ppm})$

$\begin{array}{lllllllllll}-100 & -110 & -120 & -130 & -140 & -150 & -160 & -170 & -180 & -190 & -2\end{array}$ 


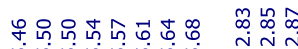

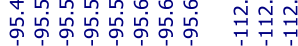

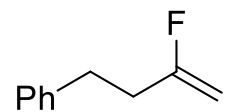

2x ${ }^{19} \mathrm{~F} \mathrm{NMR}\left(\mathrm{CDCl}_{3}, 470 \mathrm{MHz}\right)$
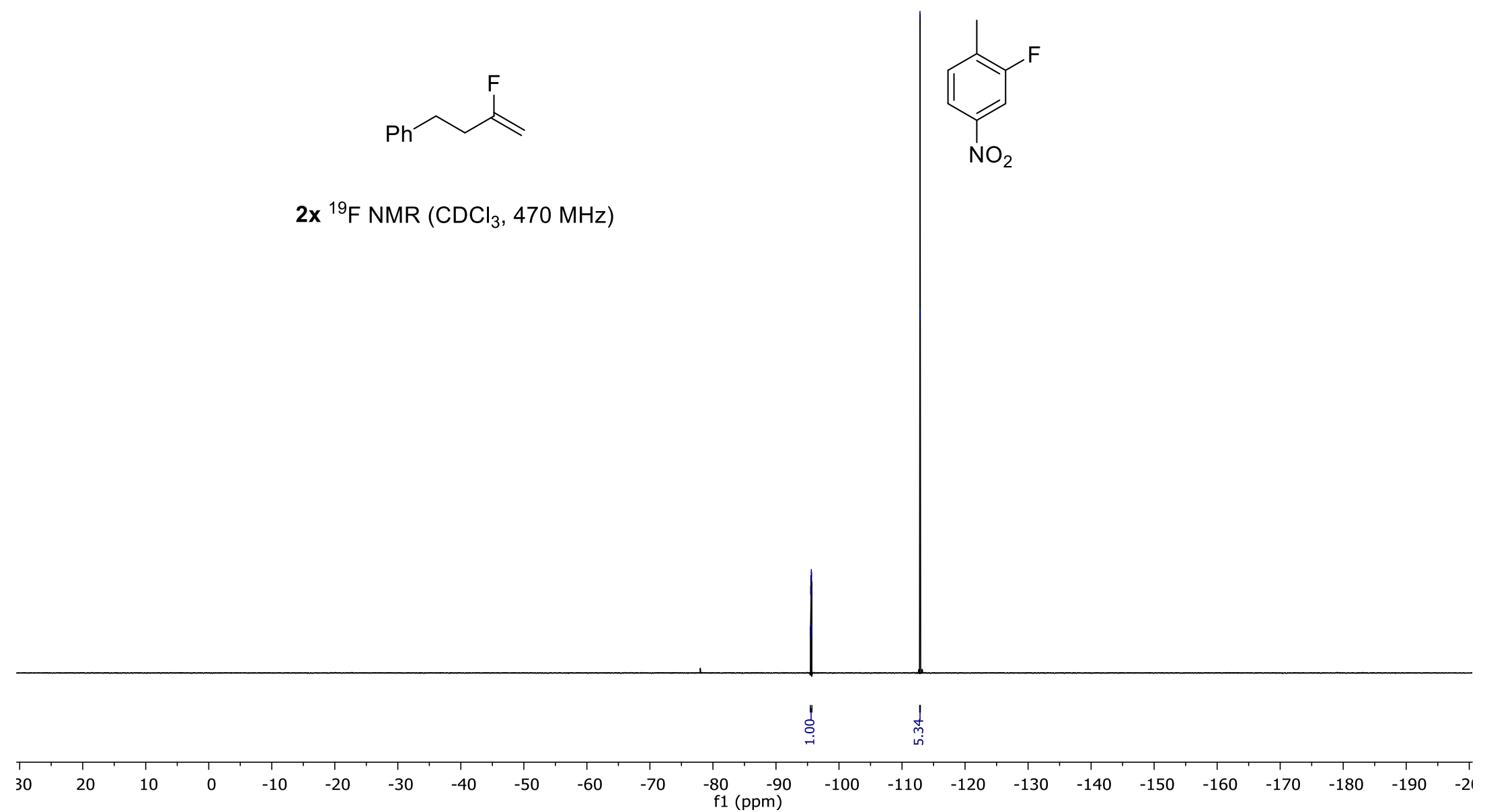


\section{ณำำㅇำ}

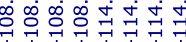

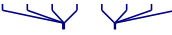
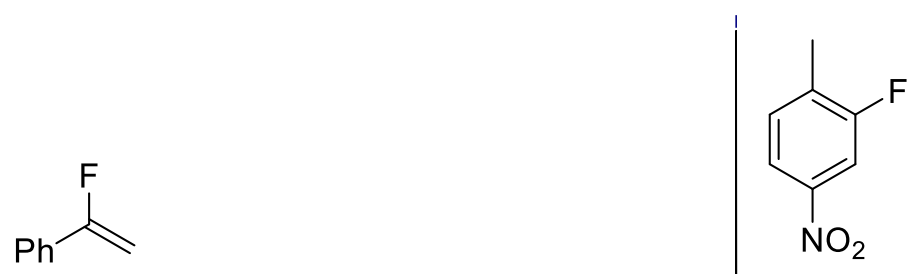

2y ${ }^{19} \mathrm{~F} \mathrm{NMR}\left(\mathrm{CDCl}_{3}, 470 \mathrm{MHz}\right)$

\begin{tabular}{lllllllllllllllllllllllllll}
\hline 30 & 20 & 10 & 0 & -10 & -20 & -30 & -40 & -50 & -60 & -70 & -80 & -90 & -100 & -110 & -120 & -130 & -140 & -150 & -160 & -170 & -180 & -190 & -2
\end{tabular}




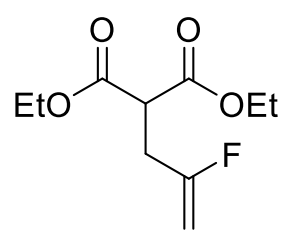

$2 z^{1} \mathrm{H}$ NMR $\left(\mathrm{CDCl}_{3}, 500 \mathrm{MHz}\right)$

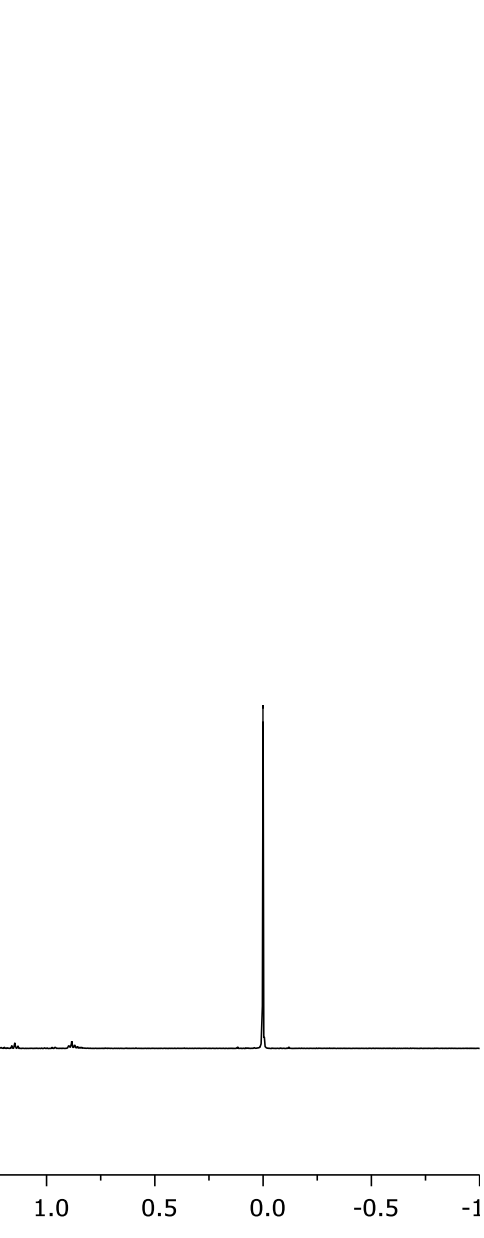




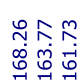

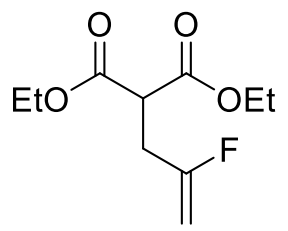

$2 z^{13} \mathrm{C} \mathrm{NMR}\left(\mathrm{CDCl}_{3}, 126 \mathrm{MHz}\right)$

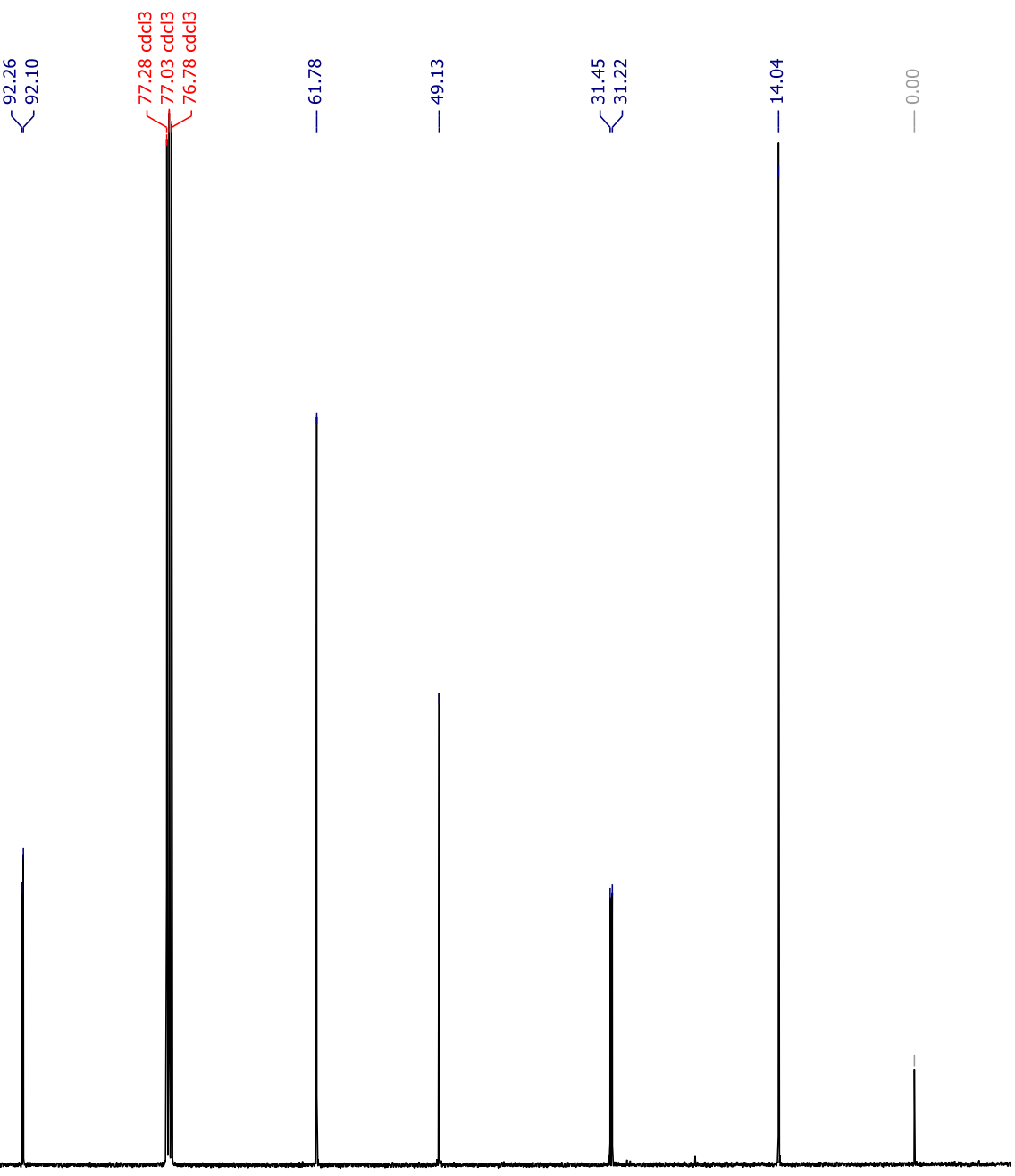

$90 \quad 180$

170

160

150

140

130

$120 \quad 110$

100

90

$80 \quad 70$

60

50

40

30 


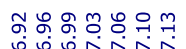

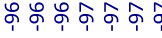

iris

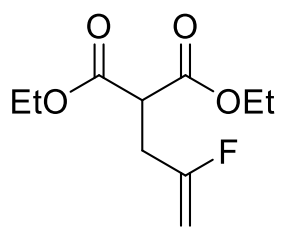

$2 \mathbf{z}^{19} \mathrm{~F} \mathrm{NMR}\left(\mathrm{CDCl}_{3}, 470 \mathrm{MHz}\right)$

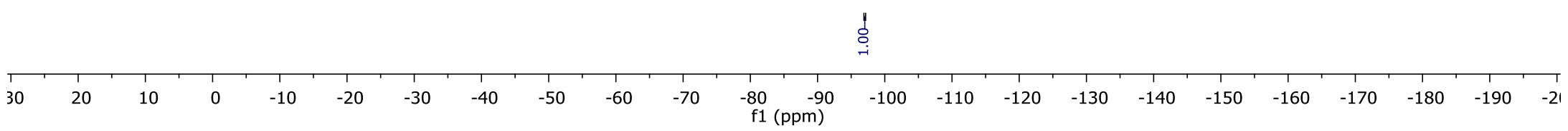




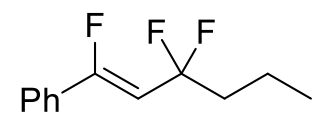

4a ${ }^{1} \mathrm{H} \mathrm{NMR}\left(\mathrm{CDCl}_{3}, 500 \mathrm{MHz}\right)$

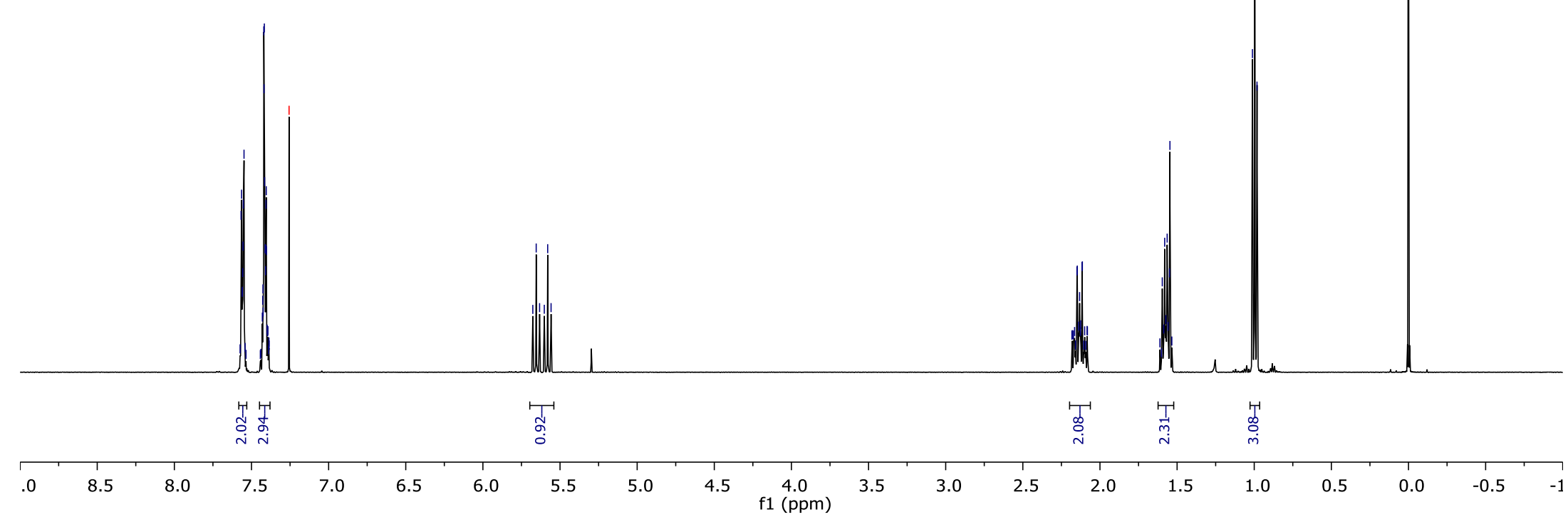



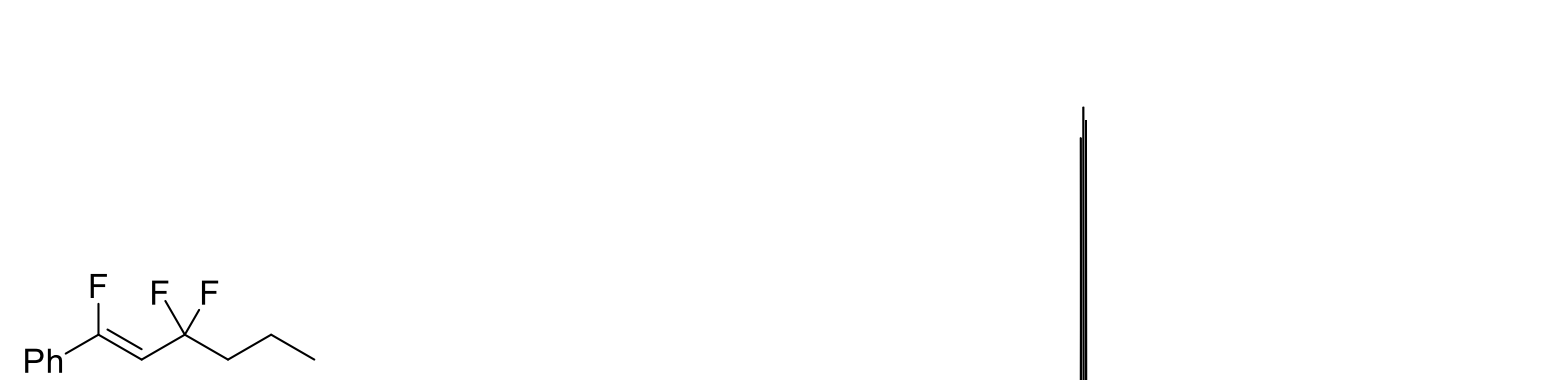

4a ${ }^{13} \mathrm{C} \mathrm{NMR}\left(\mathrm{CDCl}_{3}, 126 \mathrm{MHz}\right)$

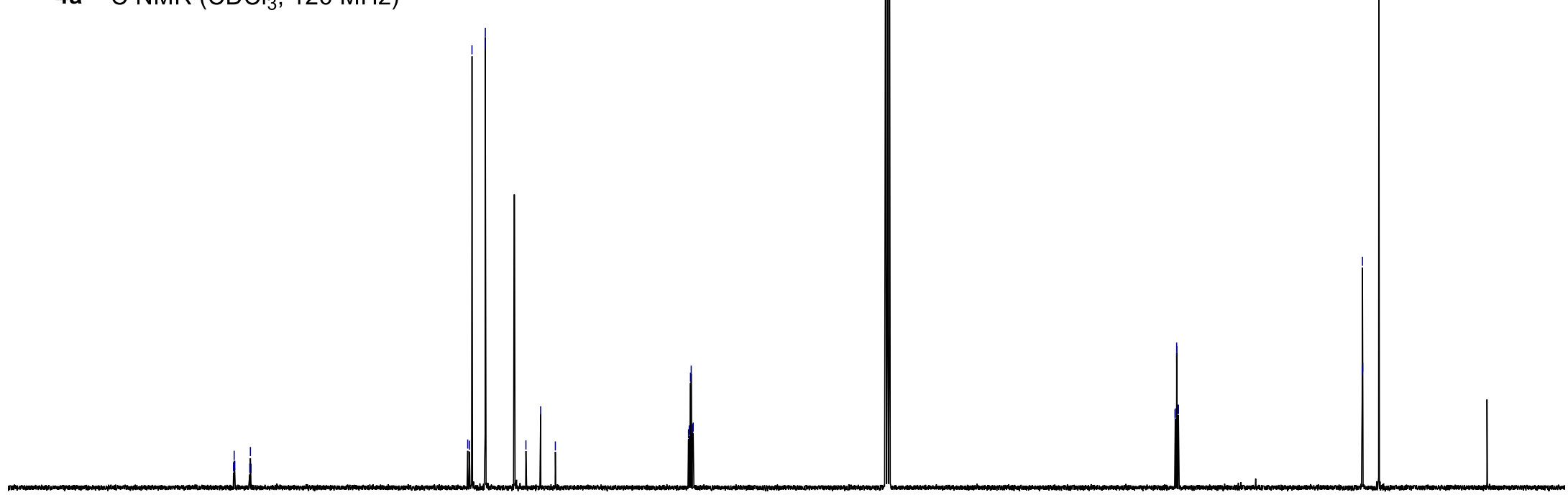




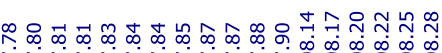

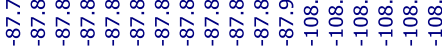

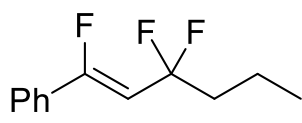

4a ${ }^{19} \mathrm{~F} \mathrm{NMR}\left(\mathrm{CDCl}_{3}, 470 \mathrm{MHz}\right)$

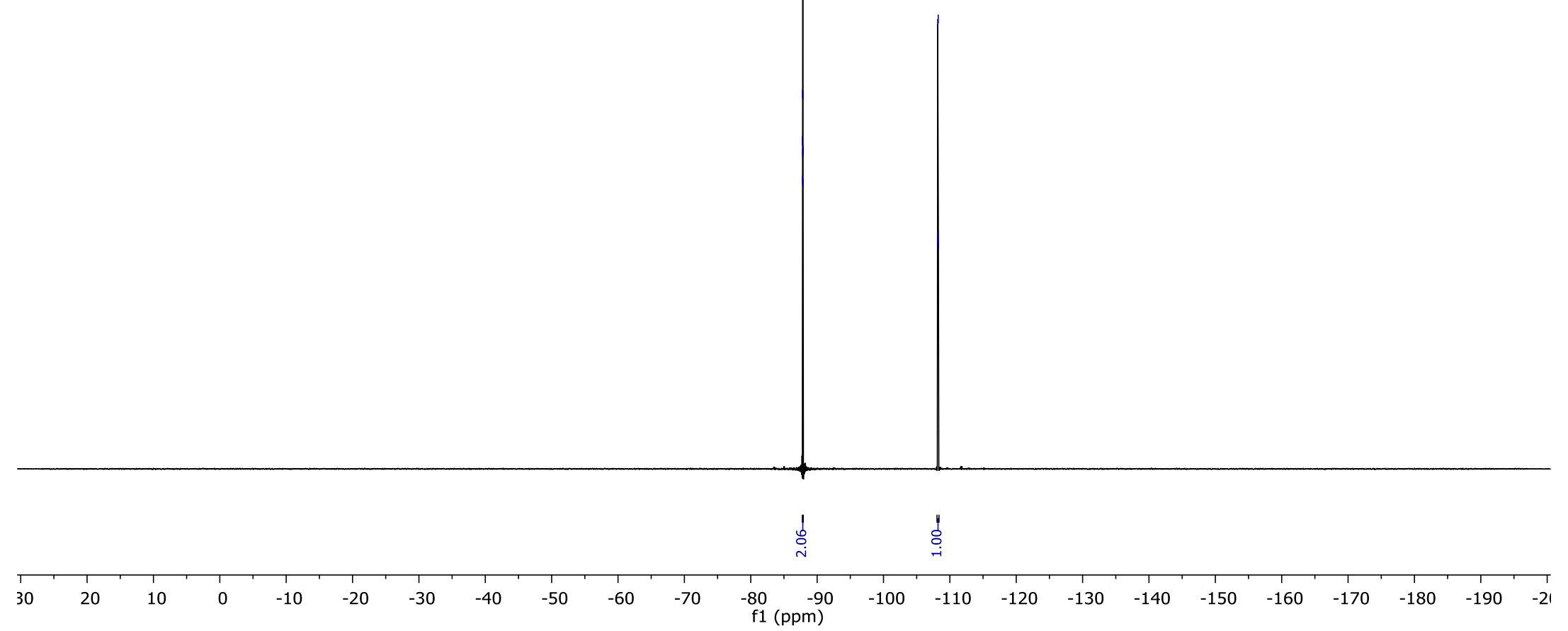




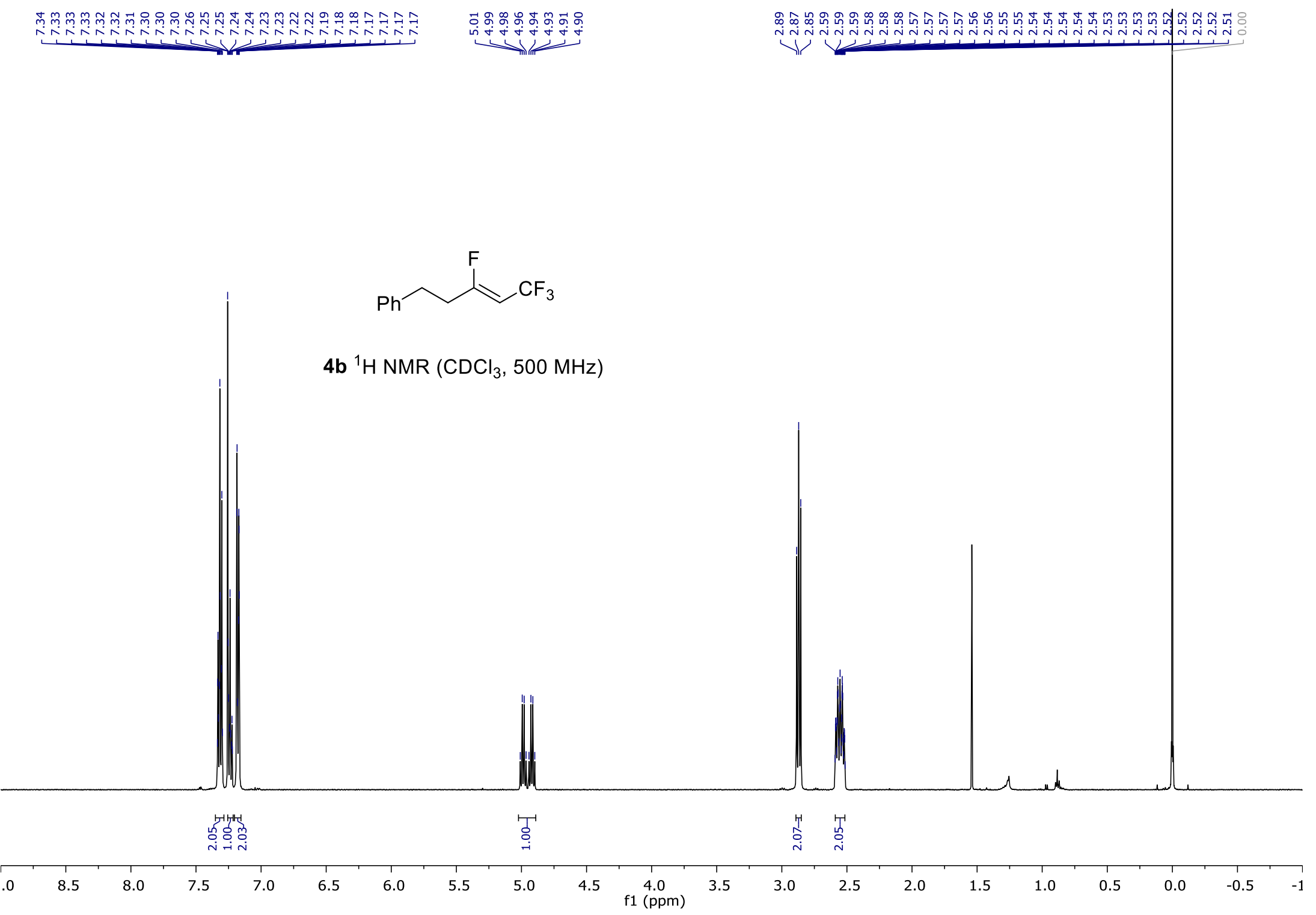




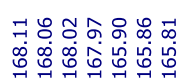

닌

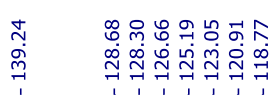

पilit

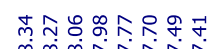

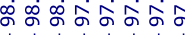

年aga

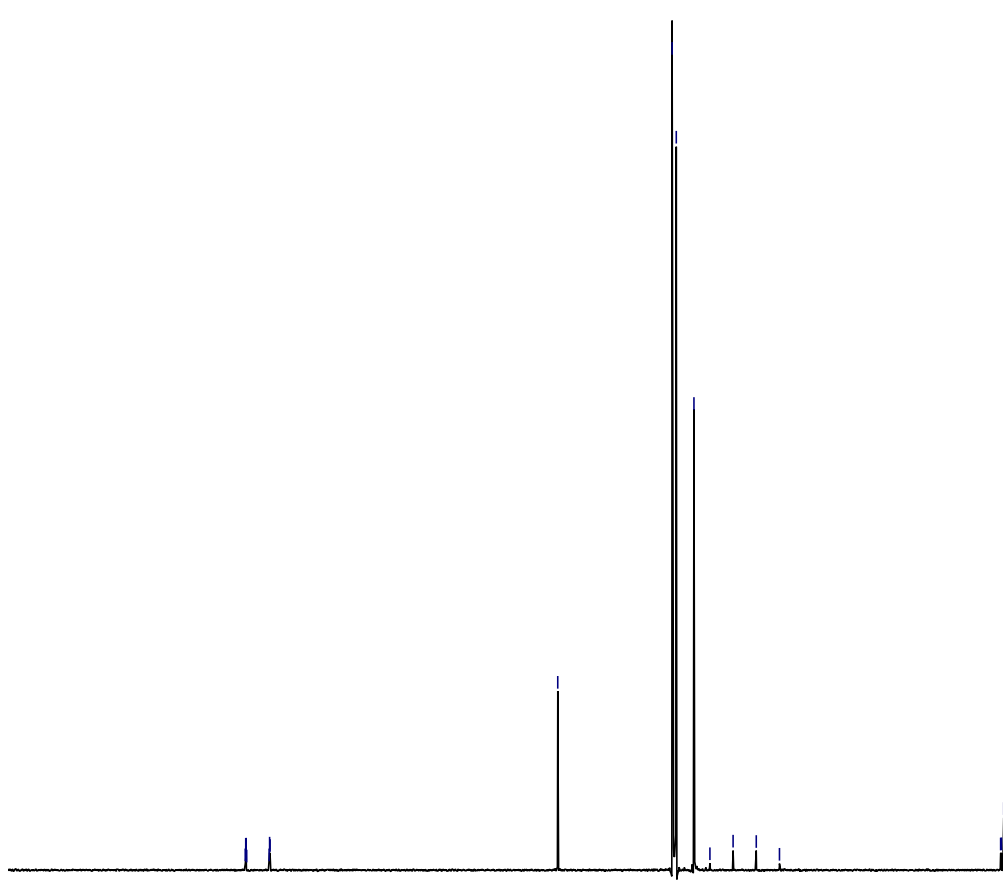

4b ${ }^{13} \mathrm{C} \mathrm{NMR}\left(\mathrm{CDCl}_{3}, 126 \mathrm{MHz}\right)$

$90 \quad 180$

160

150

140

130

$120 \quad 110$

100

90

$80 \quad 70 \quad 60$

$60 \quad 50$

$50 \quad 40$

30

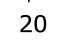

10

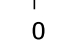


$\overrightarrow{0}$

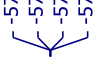

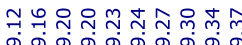

审



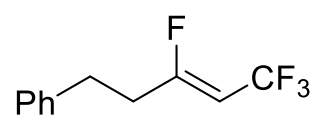

4b ${ }^{19} \mathrm{~F} \mathrm{NMR}\left(\mathrm{CDCl}_{3}, 470 \mathrm{MHz}\right)$

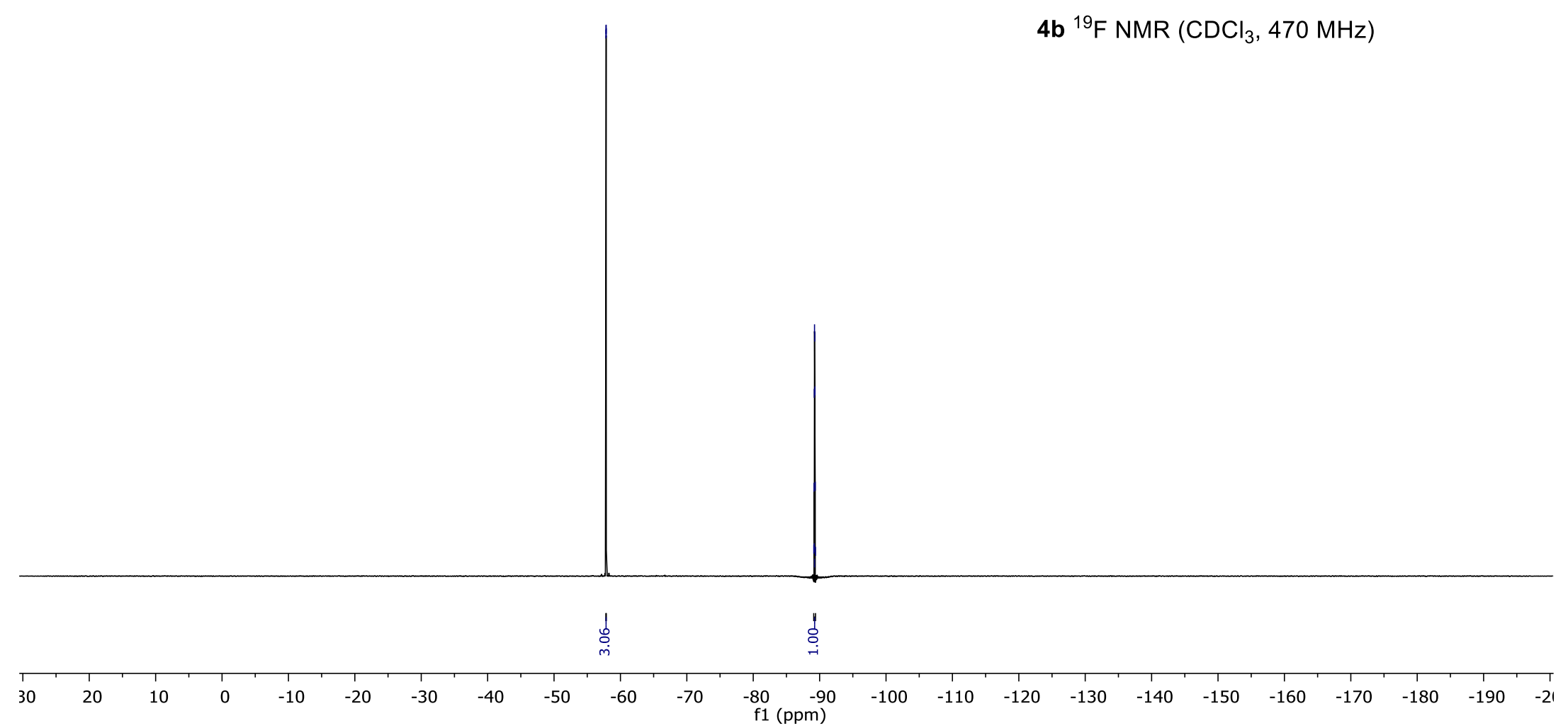



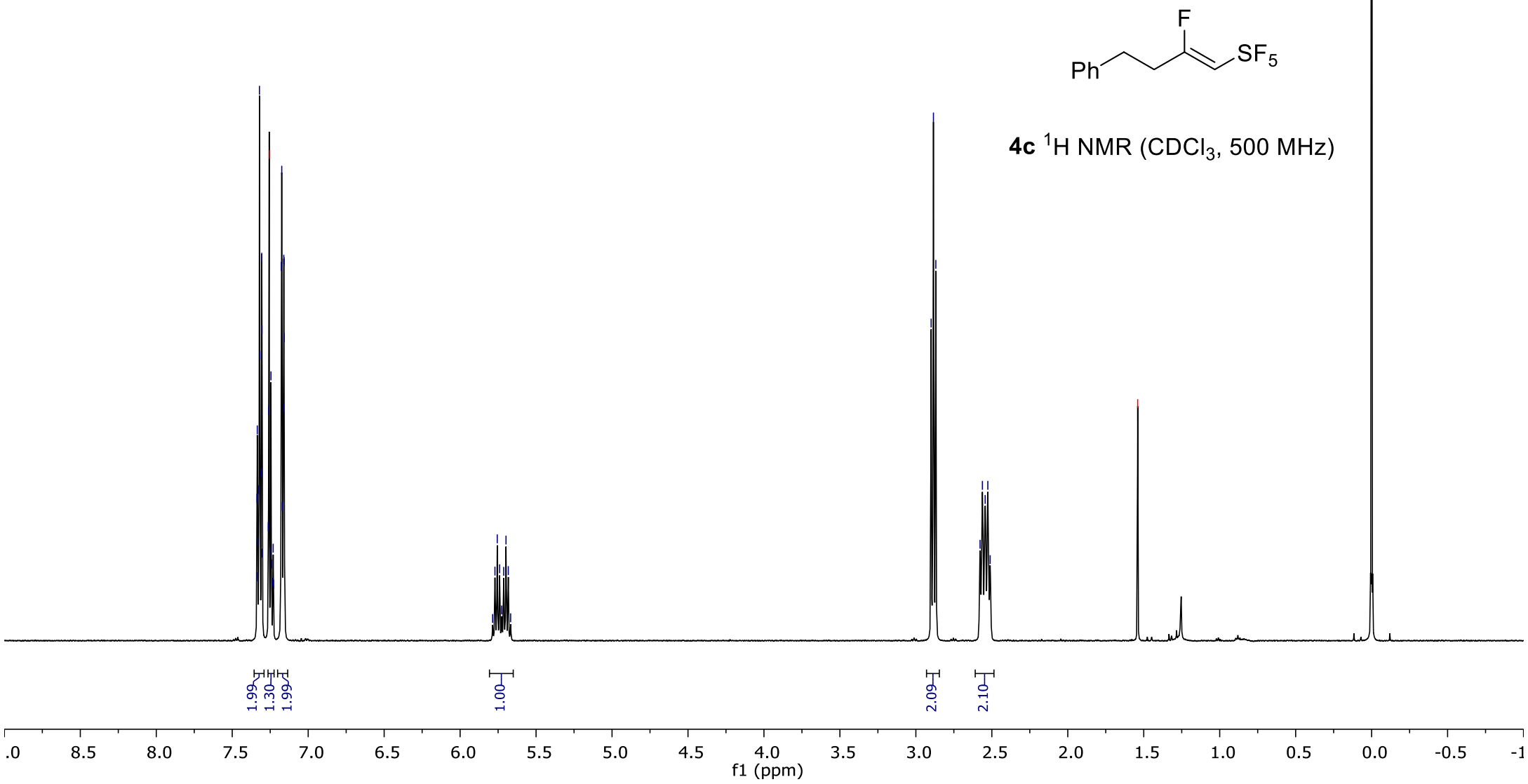


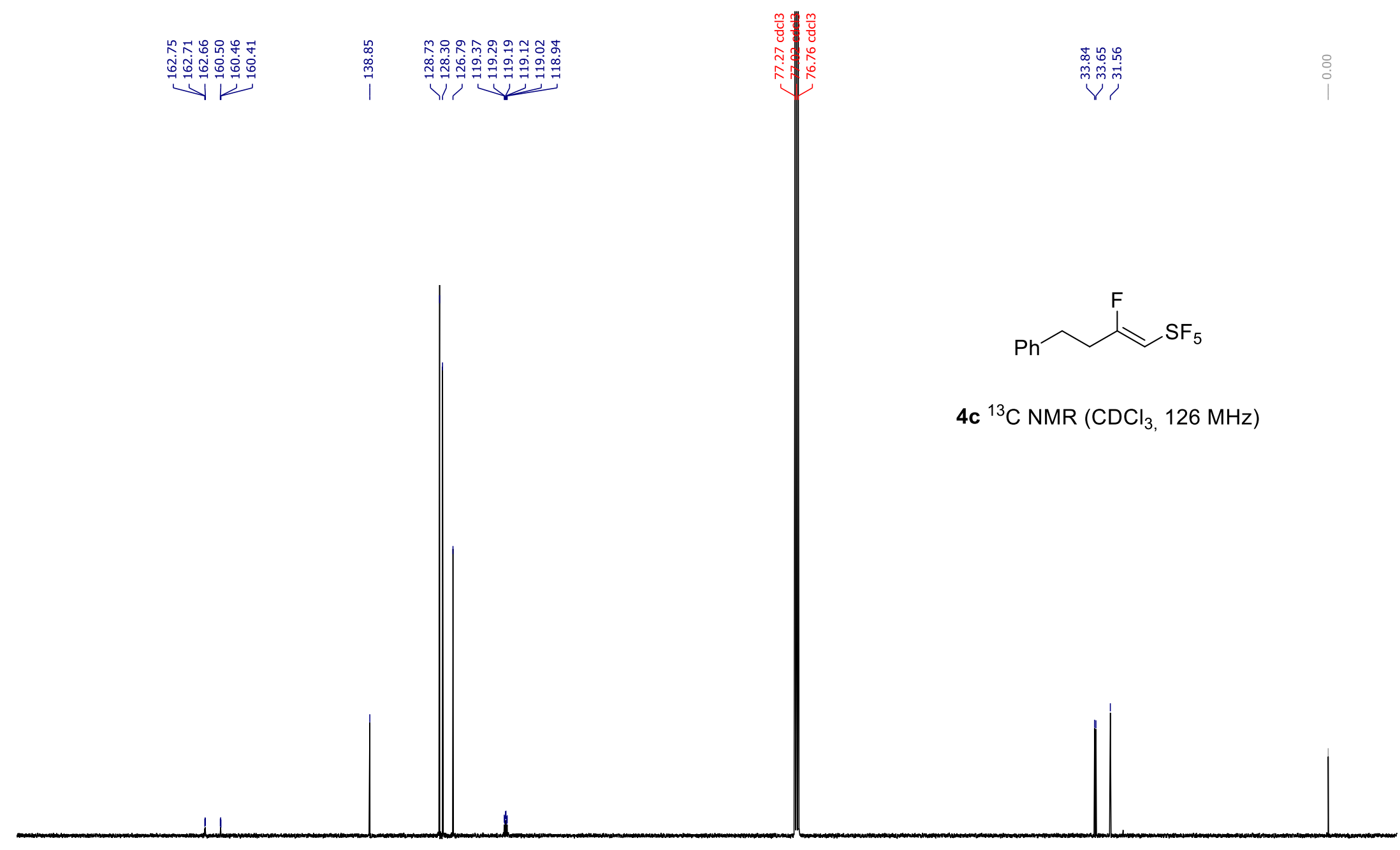

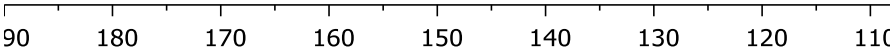
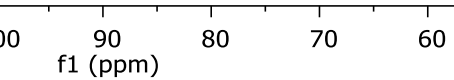

$60 \quad 50$

40

30 


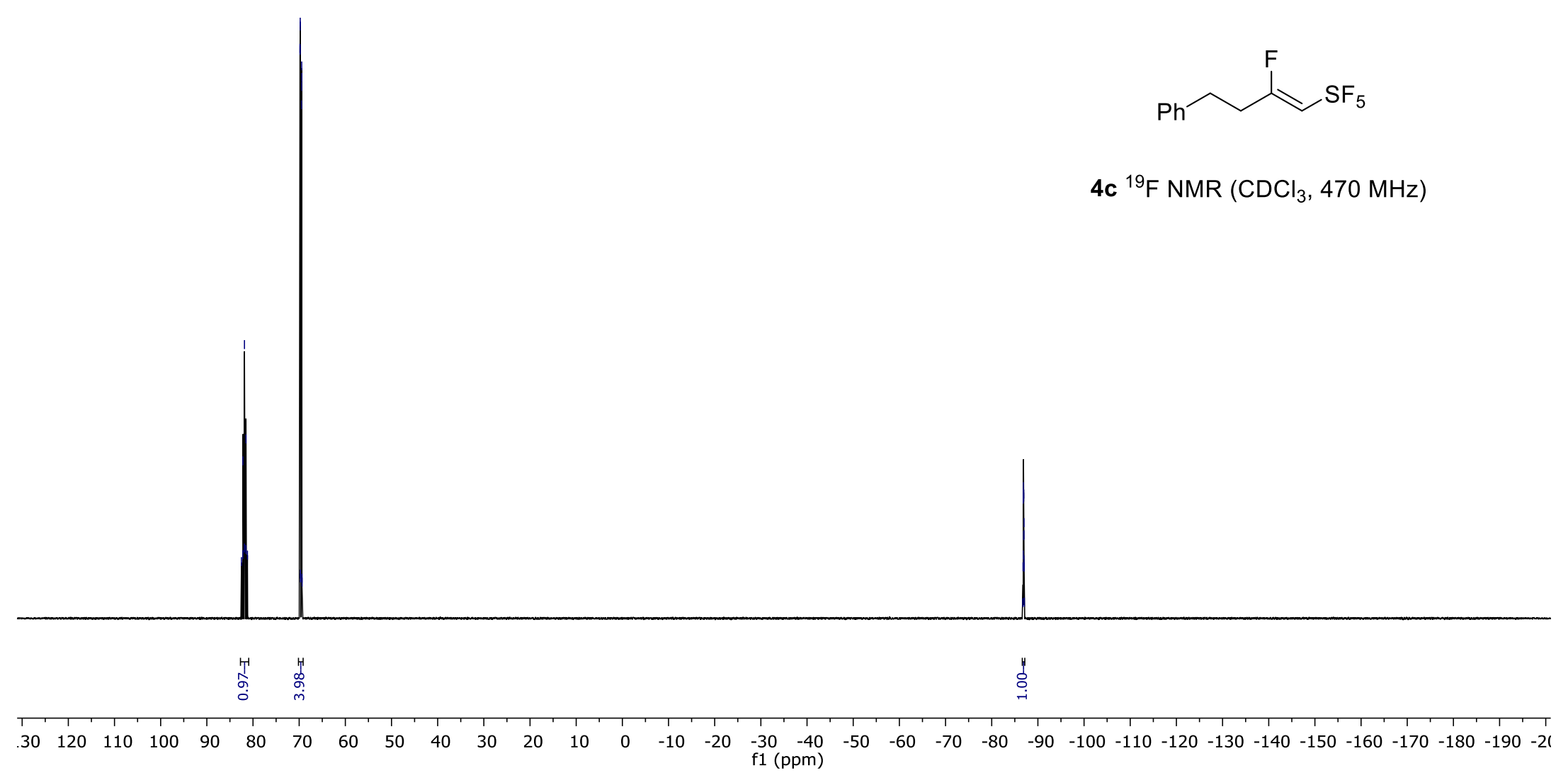

